\title{
Taxonomic affinities of the putative titanosaurs from the Late Jurassic Tendaguru Formation of Tanzania: phylogenetic and biogeographic implications for eusauropod dinosaur evolution
}

\begin{tabular}{|c|c|}
\hline Journal: & Zoological Journal of the Linnean Society \\
\hline Manuscript ID & ZOJ-05-2018-3315.R1 \\
\hline Manuscript Type: & Original Article \\
\hline Keywords: & $\begin{array}{l}\text { biogeography < Geography, dispersal < Geography, extinction < Evolution, } \\
\text { Gondwana < Palaeontology, Mesozoic < Palaeontology, Laurasia < } \\
\text { Palaeontology, Jurassic < Palaeontology, Cretaceous < Palaeontology, } \\
\text { phylogenetics < Phylogenetics }\end{array}$ \\
\hline Abstract: & $\begin{array}{l}\text { The Late Jurassic Tendaguru Formation of Tanzania, southeastern Africa, } \\
\text { records a diverse and abundant sauropod fauna, including the } \\
\text { flagellicaudatan diplodocoids Dicraeosaurus and Tornieria, and the } \\
\text { brachiosaurid titanosauriform Giraffatitan. However, the taxonomic } \\
\text { affinities of other sympatric sauropod taxa and remains are poorly } \\
\text { understood. Here, we critically reassess and redescribe these problematic } \\
\text { taxa, and present the largest phylogenetic analysis for sauropods (117 } \\
\text { taxa scored for } 542 \text { characters) to explore their placement within } \\
\text { Eusauropoda. A full re-description of the holotype of Janenschia, and all } \\
\text { referable remains, supports its validity and placement as a non- } \\
\text { neosauropod eusauropod. New information on the internal pneumatic } \\
\text { tissue structure of the anterior dorsal vertebrae of the enigmatic } \\
\text { Tendaguria tanzaniensis, coupled with a full re-description, results in its } \\
\text { novel placement as a turiasaur. A previously referred caudal sequence } \\
\text { cannot be assigned to Janenschia and displays several features that } \\
\text { indicate a close relationship with Middle-Late Jurassic East Asian } \\
\text { mamenchisaurids. It can be diagnosed by six autapomorphies, and we } \\
\text { erect the new taxon Wamweracaudia keranjei n. gen. n. sp. The Tendaguru } \\
\text { Formation shares representatives of nearly all sauropod lineages with } \\
\text { Middle Jurassic-earliest Cretaceous global faunas, but displays a greater } \\
\text { range of diversity than any of those faunas considered individually. }\end{array}$ \\
\hline
\end{tabular}




\section{Abstract}

The Late Jurassic Tendaguru Formation of Tanzania, southeastern Africa, records a rich sauropod fauna, including the diplodocoids Dicraeosaurus and Tornieria, and the brachiosaurid titanosauriform Giraffatitan. However, the taxonomic affinities of other sympatric sauropod taxa are poorly understood. Here, we critically reassess and redescribe these problematic taxa, and present the largest phylogenetic analysis for sauropods (117 taxa scored for 542 characters) to explore their placement within Eusauropoda. Janenschia robusta has played a prominent role in discussions of titanosaur origins, with various authors referring at least some remains to Titanosauria, a clade otherwise known only from the Cretaceous. Re-description of the holotype of Janenschia, and all referable remains, supports its validity and placement as a non-neosauropod eusauropod. It forms a clade with Haestasaurus, from the earliest Cretaceous of the UK, and the Middle/Late Jurassic Chinese sauropod Bellusaurus. Phylogenetic analysis and CT scans of the internal pneumatic tissue structure of Australodocus bohetii tentatively support a non-titanosaurian somphospondylan identification, making it the only known pre-Cretaceous representative of that clade. New information on the internal pneumatic tissue structure of the dorsal vertebrae of the enigmatic Tendaguria tanzaniensis, coupled with a full re-description, results in its novel placement as a turiasaur. Tendaguria is the sister taxon of Moabosaurus, from the Early Cretaceous of North America, and is the first turiasaur recognised from Gondwana. A previously referred caudal sequence cannot be assigned to Janenschia and displays several features that indicate a close relationship with Middle-Late Jurassic East Asian mamenchisaurids. It can be diagnosed by six autapomorphies, and we erect the new taxon Wamweracaudia keranjei gen. et sp. nov. The presence of a mamenchisaurid in the Late Jurassic of southern Gondwana indicates an earlier and more widespread diversification of this clade than previously realised, prior to the geographic isolation of East Asia. Our revised phylogenetic dataset sheds light on the evolutionary history of Eusauropoda, including supporting a basal diplodocoid placement for Haplocanthosaurus, and elucidating the interrelationships of rebbachisaurids. The Tendaguru Formation shares representatives of nearly all sauropod lineages with Middle Jurassic-earliest Cretaceous global faunas, but displays a greater range of diversity than any of those faunas considered individually. Biogeographic analysis indicates that the Tendaguru sauropod fauna was assembled as a result of three main phenomena during the late Early and/or Middle Jurassic: (1) invasions from Euramerica (brachiosaurids, turiasaurs); (2) endemism in west Gondwana (dicraeosaurids, diplodocids); and (3) regional extinctions that restricted the ranges of once widespread groups (mamenchisaurids, the Janenschia lineage). Multiple dispersals across the Central Gondwanan Desert are required to explain the distributions of Jurassic sauropods, suggesting that this geographic feature was at most a filter barrier that became easier to cross during the late Middle Jurassic.

Keywords: BioGeoBEARS, Biogeography, Central Gondwanan Desert, Dispersal, Extended Implied Weights, Gondwana, Mesozoic, Rebbachisauridae, Regional Extinction, Titanosauria 


\section{INTRODUCTION}

The Late Jurassic section of the Tendaguru Formation of southeastern Tanzania (Fig. 1), East Africa, preserves a rich fossil fauna, including plants (Bussert, Heinrich \& Aberhan, 2009, and references therein), invertebrates (Aberhan et al., 2002), fish (e.g. Arratia, Kriwet \& Heinrich, 2002), lissamphibians (Aberhan et al., 2002), mammals (Heinrich, 1998, 1999a, 2001), sphenodontids (Aberhan et al., 2002), squamates (Broschinski, 1999), crocodylomorphs (Heinrich et al., 2001), pterosaurs (Unwin \& Heinrich, 1999; Costa \& Kellner, 2009) and, most famously, dinosaurs (Maier, 2003). Within Dinosauria, ornithischians are represented by abundant material of the basal iguanodontian Dysalotosaurus lettowvorbecki (Virchow, 1919; Hübner \& Rauhut, 2010) and the stegosaur Kentrosaurus aethiopicus (Hennig, 1915; Galton, 1982). Theropod remains are less common: with the exception of a partial skeleton of the ceratosaur Elaphrosaurus bambergi (Janensch, 1920, 1925a; Rauhut \& Carrano, 2016), only isolated and fragmentary remains are known (Janensch, 1925a; Rauhut, 2005, 2011), although some of these are recognised as distinct taxa, i.e. Veterupristisaurus milneri (Rauhut, 2011) and Ostafrikasaurus crassiserratus (Buffetaut, 2012).

Sauropod dinosaurs are both abundant and diverse in the Tendaguru Formation (Janensch, 1929a; Heinrich, 1999b), represented by multiple skeletons of diplodocoids, i.e. the diplodocid Tornieria africana (Fraas, 1908; Remes, 2006) and the dicraeosaurids Dicraeosaurus hansemanni and D. sattleri (Janensch, 1914a; Schwarz-Wings \& Böhm, 2014), and titanosauriforms, i.e. the brachiosaurid Giraffatitan ('Brachiosaurus') brancai (Janensch, 1914a; Taylor, 2009). A second titanosauriform, Australodocus bohetii, is known only from two associated cervical vertebrae (Remes, 2007). Originally described as a second diplodocid taxon (Remes, 2007), Whitlock (2011a, b) demonstrated its titanosauriform affinities (see also D'Emic, 2012; Mannion et al., 2012) and suggested a brachiosaurid identification, whereas the analyses of Mannion et al. (2013) recovered it within the somphospondylan clade, as a titanosaur. The remaining two Tendaguru sauropod taxa have even less certain taxonomic affinities: Janenschia robusta (Fraas, 1908; Sternfeld, 1911; Wild, 1991; Bonaparte, Heinrich \& Wild, 2000) and Tendaguria tanzaniensis (Bonaparte et al., 2000).

Janenschia robusta has a complex and convoluted taxonomic history. Two sauropod skeletons (' $A$ ' and ' $B$ ') were collected from near Tendaguru Hill (Fig. 1) by a reconnaissance expedition led by Eberhard Fraas in 1907 (Fraas, 1908; Bonaparte et al., 2000; Remes, 2006). Fraas (1908) described them both as species of the new genus Gigantosaurus: G. africanus (skeleton ' $A$ ') and $G$. robustus (skeleton ' $B$ '). However, this genus name was preoccupied by 'Gigantosaurus megalonyx' from the Late Jurassic of the UK (Seeley, 1869) and so Sternfeld (1911) provided Tornieria as a replacement name, with the two Tendaguru species becoming $T$. africana and T. robusta. The German Tendaguru Expedition of 1909-1913 collected approximately 230 tonnes of additional dinosaur material (Maier, 2003), including elements from the type locality of skeleton B (Janensch, 1914b; Bonaparte et al., 2000). In his study of this material, Janensch (1922) considered the two Tornieria species to be distinct and referred T. africana (G. africanus) to the North American genus Barosaurus, creating the new combination B. africanus. Janensch (1922) also considered that the original genus name, Gigantosaurus, was now available because 'Gigantosaurus megalonyx' had been shown to be a nomen dubium by Lydekker (1888) and thus 'removed' from the literature; consequently, he revalidated $G$. robustus. However, once used, a genus name does not become available again and thus Janensch's (1922) revalidation was erroneous (Wild, 1991). As Tornieria was now considered a junior synonym of Barosaurus, and the two Tendaguru 
species are clearly distinct at higher taxonomic levels (Janensch, 1922), Wild (1991) proposed a new generic name for $T$. robusta (G. robustus): Janenschia robusta, with skeleton ' $B$ ' (a near complete hindlimb) designated as the holotype. Janensch (1914b, 1922, 1929a, 1961) referred additional material, although Bonaparte et al. (2000) and Mannion et al. (2013) concluded that only some of it could be unequivocally assigned to Janenschia.

Many authors have considered Janenschia (or at least elements at some point referred to Janenschia) to represent a titanosaur. Thus, along with potentially Australodocus (Mannion et al., 2013), it would represent the earliest and only pre-Cretaceous body fossil occurrence of this radiation of derived sauropods (Janensch, 1929a; Mclntosh, 1990; Wild, 1991; Jacobs et al., 1993; Upchurch, 1995; Salgado \& Calvo, 1997; Wilson \& Sereno, 1998; Upchurch, Barrett \& Dodson, 2004; Curry Rogers, 2005; Mannion \& Calvo, 2011). However, Bonaparte et al. (2000) disputed the titanosaurian affinities of Janenschia and instead suggested close affinities with the basal macronarian Camarasaurus from the Late Jurassic of North America. Royo-Torres \& Cobos (2009) proposed that some of the material included in Janenschia might be referable to the non-neosauropod eusauropod clade Turiasauria (see also Britt et al., 2017), otherwise known only from western Europe (Royo-Torres, Cobos \& Alcalá, 2006) and North America (Royo-Torres et al., 2017a). D’Emic (2012) suggested titanosauriform affinities for Janenschia, but noted the absence of unambiguous titanosaurian features. Recently, two independent phylogenetic analyses have provided support for a non-titanosaurian position, placing Janenschia as a non-titanosauriform macronarian (Carballido et al., 2011; Mannion et al., 2013). In addition, Mannion et al. (2013) treated a previously referred caudal sequence (MB.R.2091.1-30) as a separate operational taxonomic unit, recovering this taxon as a basal eusauropod, with potentially close affinities to the contemporaneous Chinese sauropod Mamenchisaurus.

Bonaparte et al. (2000) named Tendaguria tanzaniensis on the basis of two associated dorsal vertebrae, and a tentatively referred cervical vertebra from a different locality, all material originally assigned to Janenschia (Janensch, 1929a). Bonaparte et al. (2000) were unable to assign it to any existing group within Sauropoda; instead they erected the new monospecific family Tendaguriidae. Upchurch et al. (2004) listed Tendaguria under Sauropoda incertae sedis and commented upon the unusual combination of basal and derived sauropod features, and Mannion \& Calvo (2011) listed it as a putative titanosaur. Carballido et al. (2011) were the first to include Tendaguria in a phylogenetic analysis, recovering it as either a basal macronarian or non-neosauropod eusauropod, although in an updated version of this matrix it was recovered as a non-titanosaurian somphospondylan (Carballido et al. 2012, 2015; Carballido \& Sander, 2014). A non-neosauropod eusauropod position was partly supported by the analyses of Mannion et al. (2013), although Tendaguria was recovered as a basal diplodocoid in some of their trees.

As such, it is clear that much confusion and controversy still surrounds the systematic positions and taxonomy of these important, but enigmatic taxa. Here, we fully re-describe Janenschia, Tendaguria and the MB.R.2091.1-30 tail, as well as all remains previously referred to Janenschia, including discussion of referred material described from the Late Jurassic of Zimbabwe (Raath \& McIntosh, 1987). We also present new CT data on the internal pneumatic tissue structure of Tendaguria, as well as the sympatric sauropod genus Australodocus, which helps clarify the taxonomic affinities of the latter within Titanosauriformes. Additionally, we explore the phylogenetic placement of all of these problematic taxa in an extensively revised and expanded eusauropod data matrix. Lastly, we carry out a biogeographic analysis to examine patterns among Middle Jurassic-Early Cretaceous sauropod faunas, placing the Tendaguru sauropod fauna in a global context. 
Institutional abbreviations: AMNH, American Museum of Natural History, New York, USA; BYU, Brigham Young University, Museum of Paleontology, Provo, Utah, USA; CM, Carnegie Museum of Natural History, Pittsburgh, Pennsylvania, USA; CMNH, Cleveland Museum of Natural History, Cleveland, Ohio, USA; CPT, Museo de la Fundación Conjunto Paleontológico de Teruel-Dinópolis, Aragón, Spain; IVPP, Institute of Vertebrate Paleontology and Paleoanthropology, Beijing, China; LGP, Lingwu Geopark, Lingwu, Ningxia Hui Autonomous Region, China; LM, Lingwu Museum, Lingwu, Ningxia Hui Autonomous Region, China; MACN, Museo Argentino de Ciencias Naturales 'Bernardino Rivadavia', Buenos Aires, Argentina; MB.R., Collection of Fossil Reptiles in the Museum für Naturkunde Berlin, Germany; MCNV, Museo de Ciencias Naturales de Valencia, Spain; MDS, Museo de Dinosaurios de Salas de los Infantes, Burgos, Spain; MfN, Museum für Naturkunde Berlin, Germany; MG, Museu Geológico do Instituto Geológico e Mineiro, Lisbon, Portugal; MIWG, Museum of Isle of Wight Geology (now Dinosaur Isle Visitor Centre), Isle of Wight, United Kingdom; ML, Museu da Lourinhã, Portugal; MLP, Museo de La Plata, La Plata, Argentina; MMCH, Museo Municipal 'Ernesto Bachman', Villa El Chocón, Neuquén,Argentina; MN, Museu Nacional, Rio de Janeiro, Brazil; MNHN, Muséum National d'Histoire Naturelle, Paris, France; MNN, Musée National du Niger, Niamey, Republic of Niger; MOZ, Museo Provincial de Ciencias Naturales 'Prof. Dr. Juan A. Olsacher', Zapala, Neuquén, Argentina; MPCA, Museo Provincial Carlos Ameghino, Cipolletti, Río Negro, Argentina; MPEF, Museo Paleontológico Egidio Feruglio, Trelew, Argentina; MPZ, Museo Paleontológico de la Universidad de Zaragoza, Spain; MUCPv, Museo de Geología y Paleontología de la Universidad Nacional del Comahue, Neuquén, Argentina; NHMUK, Natural History Museum, London, UK; PVL, Colección de Paleontología de Vertebrados de la Fundación Instituto Miguel Lillo, Tucumán, Argentina; SMNS, Staatliches Museum für Naturkunde Stuttgart, Germany; UFRJ-DG, Universidade Federal do Rio de Janeiro, Departamento de Geologia, Brazil; UMNH, Natural History Museum of Utah, USA; UNPSJB, Universidad Nacional de la Patagonia San Juan Bosco, Comodoro Rivadavia, Argentina; WDC, Wyoming Dinosaur Center, Thermopolis, Wyoming, USA.

Anatomical abbreviations: ACDL, anterior centrodiapophyseal lamina; aEI, average Elongation Index (centrum length [excluding condyle] divided by the mean average value of the posterior mediolateral width and dorsoventral height); CCl, Condylar Convexity Index (anteroposterior length of posterior condylar ball divided by mean radius of the condyle [i.e. mediolateral width + dorsoventral height of articular surface, divided by 4]); CDF, centrodiapophyseal fossa; CPOL, centropostzygapophyseal lamina; CPRL, centrprezygapophyseal lamina; EPRL, epipophyseal-prezygapophyseal lamina; ISPRL, lateral spinoprezygopophyseal lamina; PCDL, posterior centrodiapophyseal lamina; PCPL, posterior centroparapophyseal lamina; PODL, postzygodiapophyseal lamina; PPDL, paradiapophyseal lamina; PRCDF, prezygapophyseal centrodiapophyseal fossa; PRDL, prezygodiapophyseal lamina; SDF, spinodiapophyseal fossa; SPDL, spinodiapophyseal lamina; SPOL, spinopostzygapophyseal lamina; SPRL, spinoprezygapophyseal lamina; TPOL, interpostzygapophyseal lamina; TPRL, interprezygapophyseal lamina.

CT scanning: CT scans of the specimens MB.R.2092.1-2 (Tendaguria), MB.R.2091.31 (cf. Tendaguria), and MB.R.2454 and MB.R.2455 (Australodocus) were performed with a 320section multidetector CT unit (Aquillion ONE; Toshiba, Otawara, Japan) at the Charité Universitätsmedizin Berlin (Campus Charité Mitte) in Germany. A helical scan mode with a 
rotation time of one second was chosen. The tube voltage was set to $135 \mathrm{kVp}$, and a tube current of $450 \mathrm{~mA}$ was used. Axial images were acquired with a section thickness of 500 micrometres $(0.5 \mathrm{~mm})$; subsequently, secondary multiplanar reconstructions were calculated in axial, coronal, and sagittal orientations, each with a section thickness of $3 \mathrm{~mm}$. Imaging of the data (bone algorithm) was performed with the software OsiriX2 (v. 5.5.1, 32 bit, open source version).

\section{SYSTEMATIC PALAEONTOLOGY}

SAUROPODA MARSH, 1878

EUSAUROPODA UPCHURCH, 1995

JANENSCHIA WILD, 1991

Type species: Janenschia robusta (Fraas, 1908)

Gigantosaurus robustus Fraas, 1908

Tornieria robusta Sternfeld, 1911

Gigantosaurus robustus Janensch, 1922

Barosaurus robustus Haughton, 1928

Tornieria robusta Janensch, 1961

Holotype: SMNS 12144 - associated right hindlimb comprising the distal end of the femur, tibia, fibula, astragalus, and complete pes.

Topotypes: left pubis (MB.R.2090.2 [B8]), right ischium (MB.R.2090.4 [B13]) and distal half of left tibia (MB.R.2090.1 [B6]) from the same locality as the holotype (Janensch, 1914b; Bonaparte et al., 2000; Mannion et al., 2013). A second, slightly larger, right ischium (MB.R.2090.3 [B11]), indicates the presence of a second individual.

Type locality and horizon: Quarry B, approximately $900 \mathrm{~m}$ southeast of Tendaguru Hill, Lindi District, southeastern Tanzania (Fig. 1); Upper Dinosaur Member (Upper Saurian Bed), Tendaguru Formation; Tithonian, Late Jurassic (Fraas, 1908; Janensch, 1914b, 1925b, 1929a; Wild, 1991; Bonaparte et al., 2000; Aberhan et al., 2002; Remes, 2006; Bussert et al., 2009).

Referred material: All of the forelimb and hindlimb material from Quarry P (MB.R.2095 2245 and 2707), at Nterego, approximately $1.2 \mathrm{~km}$ northeast of Tendaguru Hill, Lindi District, southeastern Tanzania (Fig. 1); Upper Dinosaur Member (Upper Saurian Bed), Tendaguru Formation; Tithonian, Late Jurassic (Janensch, 1922, 1929a, 1961; Heinrich, 1999b; Bonaparte et al., 2000; Aberhan et al., 2002; Bussert et al., 2009). Note that a number of these elements are now lost (see below).

Revised diagnosis: Janenschia can be diagnosed by two autapomorphies (marked with an asterisk), as well as one local autapomorphy: (1) tibia with prominent tubercle ('tuberculum fibularis') on the posterior surface of the distal half of the cnemial crest; (2) extremely prominently developed projection posteromedial to the ascending process of the astragalus*; and (3) high metatarsal I to V length ratio (1.34)*. 
Additional comments: A sacrum and ilium were also apparently discovered at the type locality (Janensch, 1929a), but were either subsequently destroyed, or were never collected. However, it is not entirely clear whether the missing sacrum and ilium are those discussed and photographed in Fraas (1908: p. 126-128), or newly discovered elements. If the former is correct, as suggested in Janensch (1961: p. 201), then these elements did not come from the same locality as the holotype and the topotypic materials (Fraas, 1908), and cannot be confidently attributed to Janenschia. Regardless, no anatomical information can be gleaned from these elements and thus we do not discuss them further. Bonaparte et al. (2000) noted that Janensch's field catalogue also mentioned a partial forefoot from the type locality, but there is no further information on this material and it was presumably either destroyed or never collected. An alternative possibility is that this is MB.R.2093, a near complete, articulated right manus that was referred to Janenschia (Janensch, 1922). However, Janensch $(1922,1961)$ stated that this was collected from near to the Janenschia type locality, rather than from the type locality itself. Furthermore, the surrounding area also yielded the type skeleton of Tornieria, and so we cannot be certain that MB.R.2093 belongs to Janenschia. As such, we exclude MB.R.2093 from Janenschia and describe it separately (see below). Other previously referred remains also cannot unequivocally be assigned to Janenschia and are treated separately too.

\section{DESCRIPTION AND COMPARISONS OF THE HOLOTYPE MATERIAL OF JANENSCHIA ROBUSTA}

\section{Femur}

Only the distal end of the right femur is preserved (Fig. 2; see Table 1 for measurements). The uppermost preserved portion shows that the shaft was anteroposteriorly compressed. The distal articular surface is strongly convex anteroposteriorly, such that it curves up slightly onto the anterior surface and, much more prominently, onto the posterior surface of the femur. The posterior surface forms a deep, transversely oriented concavity between the two distal condyles, whose transverse widths are subequal. This differs from the condition in most titanosauriforms, whereby the fibular condyle is considerably wider (Wilson, 2002; Poropat et al., 2016). A deep groove also divides the fibular condyle into larger medial, and smaller lateral portions, as is the case in most derived eusauropods, with the exception of some titanosaurs (Poropat et al., 2016), e.g. Saltasaurus (PVL 4017-79: PDM \& PU pers. obs. 2013). No additional ridge is present within this groove. The tibial condyle extends further posteriorly and slightly more distally than the fibular condyle, although the latter is difficult to fully assess as a consequence of the incomplete nature of the femur. The anterior surface of the femur is gently concave transversely along its central third, with this developing into a deep concavity distally. The anterior two-thirds of the distal articular surface directly beneath this concavity slopes to face anteroventrally; consequently, it does not extend as far distally as the posterior third of the distal surface. The medial surface of the femur is flat and faces medially and slightly dorsally, whereas the lateral surface is anteroposteriorly convex, except for a shallow concavity directly anterior to the lateral edge of the fibular condyle. This region is slightly damaged, but the concavity appears to be genuine.

\section{Tibia}

The proximal end of the complete right tibia (Fig. 3) is only slightly expanded mediolaterally, and it is along the posterior half that much of this widening occurs (see Table 1 for measurements). Its rugose proximal articular surface is uneven, with the lateral half 
convex and the medial half concave (anteroposteriorly and mediolaterally, in both cases). The cnemial crest projects laterally, curving slightly posteriorly towards its lateral margin. A prominent, 'bulge'-like tubercle is present on the posterior surface of the distal half of the cnemial crest. This tubercle extends approximately as far laterally as the cnemial crest, with the lateral margins of each separated by a gentle, proximodistally elongate groove. The tubercle and cnemial crest merge into one another close to the point where the cnemial crest fades out into the tibial shaft. This feature was described as the 'tuberculum fibularis' in the dicraeosaurid Suuwassea, and was noted as also present in several diplodocids (Harris, 2007; Tschopp, Mateus \& Benson, 2015), including Tornieria (MB.R.2572: PDM pers. obs. 2014). A comparable tubercle is also known in the brachiosaurids Giraffatitan and Vouivria (Mannion, Allain \& Moine, 2017), but has not been reported in other non-flagellicaudatan sauropod tibiae. As such, it is here regarded as a local autapomorphy of Janenschia.

Approximately one-third of the distance from the posterior margin of the proximal end, there is a pinched out proximolateral projection, which Bonaparte et al. (2000: p. 37) described as a 'second cnemial crest'. This projection is separated from the true cnemial crest by a large ' $U$ '-shaped groove. Bonaparte et al. (2000) regarded this 'second cnemial crest' as an autapomorphy of Janenschia; however, this feature is much more widespread among sauropods, although it is absent in many somphospondylans (Mannion et al., 2013).

At midshaft, the long axis of the cross section through the tibial shaft is oriented along an anterolateral-to-posteromedial axis, with the axis perpendicular to this strongly compressed. The anterolateral and posteromedial surfaces of the tibial shaft are flat. Throughout the length of the tibia, the medial surface is much broader anteroposteriorly than the lateral one, with the latter forming an almost rounded ridge. A gentle, rounded, proximodistally elongate ridge extends a short distance upwards along the posteromedial surface, close to the anteromedial margin; this begins approximately $150 \mathrm{~mm}$ from the distal end.

The anterior surface of the distal end forms a subtriangular region. This area is predominantly flat, but becomes transversely convex towards its lateral edge. No anterolateral ridge is formed, contrasting with that seen in some titanosaurs, e.g. Bonatitan (MACN 821 and 1061: PDM \& PU pers. obs. 2013) and Diamantinasaurus (Poropat et al., 2015a). The medial surface of the distal end faces posteromedially: it is flat along its anterior half, but becomes transversely convex as it curves round to form the medial half of the posterior surface. The posterolateral surface of the distal end is also transversely convex. The medial malleolus projects further posteriorly and distally than the lateral one; although slightly damaged, the medial malleolus clearly did not expand far laterally. The rugose distal articular surface is convex in all directions along its medial third. The middle third of the distal surface is strongly concave transversely and very mildly concave anteroposteriorly. Beneath the lateral malleolus, the distal surface is fairly flat, but slopes so that it faces posteroventrally and very slightly medially.

\section{Fibula}

The proximal end of the complete right fibula (Fig. 4) is transversely compressed and anteroposteriorly elongate, with a rugose and convex proximal articular surface (see Table 1 for measurements). It lacks the anteromedial crest seen in many somphospondylans (Wilson \& Upchurch, 2009; D’Emic, 2012; Mannion et al., 2013). Although poorly preserved, the medial surface of the proximal end has the subtriangular, striated area that characterises derived eusauropods (Wilson \& Sereno, 1998), but that is apparently lost in some titanosauriforms (D'Emic, 2012). The lateral surface of the proximal half of the fibula is 
anteroposteriorly convex, whereas the corresponding section of the medial surface is flat to very mildly concave.

On the medial surface, a gentle ridge extends steeply anteroventrally from the posteromedial margin, beginning at approximately $180 \mathrm{~mm}$ from the proximal end and extending to midlength of the fibula. A rugose tuberosity is situated approximately onequarter of the length from the proximal end, and is located predominantly on the anterior surface, but swells outwards to also form an anteromedial projection. This appears to be equivalent to the 'tuberculum tibialis' described in the fibula of Suuwassea (Harris, 2007). The lateral trochanter begins at approximately the same level as the distal end of the tuberculum tibialis and extends distally to approximately the midlength of the fibula. It occupies almost the entire lateral surface over its near $200 \mathrm{~mm}$ extent, pinching out proximally and distally to form an ellipse in lateral view. A fairly prominent ridge forms the posterior margin of the lateral trochanter, whereas a weakly developed ridge forms the anterior margin. The area in between these ridges is strongly striated and rugose, but is not excavated; as such, the lateral trochanter does not form the double ridge structure seen in many somphospondylans, e.g. Gobititan (You, Tang \& Luo, 2003) and Tastavinsaurus (Canudo, Royo-Torres \& Cuenca-Bescós, 2008). The lateral trochanter does not project beyond the lateral margin of the remainder of the fibula, contrasting with the hypertrophied lateral trochanters seen in several derived titanosaurs, e.g. Laplatasaurus (Powell, 2003).

Distal to the lateral trochanter, the lateral surface is fairly flat, becoming transversely convex along the distal quarter of the fibula. The distal third of the medial surface is strongly concave in both directions for reception of the tibia, and a prominent ridge forms the posteromedial border of this concavity. A ridge also extends along the anterior margin of the distal third of the fibula, although this is mainly coated in plaster. At its distal end, the fibula expands a little posteriorly and strongly medially, and the distal articular surface is rugose and convex in all directions.

Tarsus

When articulated, the complete right astragalus (Fig. 5; see Table 1 for measurements) caps all of the distal end of the tibia, contrasting with the condition in most titanosauriforms, whereby the medial portion of the distal end of the tibia remains uncapped (Wilson \& Upchurch, 2009; Ksepka \& Norell, 2010; Mannion et al., 2013). The rugose anterior surface of the astragalus lacks foramina and curves into the distal surface. The distal surface is strongly convex anteroposteriorly, but less so transversely, only developing a convexity in this orientation along the medial third. Medially, the astragalus shows a prominent decrease in proximodistal height, with this reduction mainly occurring along the distal margin. The ascending process extends to the posterior margin of the astragalus, as in all neosauropods (Wilson \& Sereno, 1998), as well as Bellusaurus (Mo, 2013). The proximal surface of the ascending process of the Janenschia astragalus is flat, with the exception of an upturned posteromedial corner. However, this upturned region has been re-attached after breakage and is partly reconstructed; therefore, it might be purely artefactual.

The lateral surface of the astragalus is concave in all directions for reception of the fibula. It is not deflected posterolaterally, contrasting with the morphology seen in most diplodocoid astragali (Whitlock, 2011a). There is no distolateral lip, a feature which is present in many sauropods, but is lost in the majority of macronarians (Mannion et al., 2013). Within this lateral concavity there is a prominent fossa situated at approximately midlength, close to the proximal margin. This fossa is pierced by a small foramen. A 
shallower, unpierced fossa is also present at midheight of the lateral surface, close to the posterior margin.

Posteromedial to the ascending process, there are three fossae on the proximal surface of the astragalus. The largest fossa is oval shaped, with a diameter of $40 \mathrm{~mm}$, whereas the other two are smaller and subcircular. Both the largest fossa and the medial one face mainly posteriorly, whereas the most posterior fossa faces dorsally. A short ridge extends anteromedially from the posteromedial margin of the posterior fossa. Medial to this ridge is a large, shallow, dorsally-facing fossa, which is itself excavated by three small fossae.

The posterior surface of the ascending process is concave in both directions. This is in part caused by the strong posterior expansion of the astragalus, directly posterior to the most medial fossa. A 'tongue'-like projection posteromedial to the ascending process, separated from the latter by a groove, is the plesiomorphic eusauropod condition, but this feature is greatly reduced or lost in most titanosauriforms (D'Emic, 2012; Mannion et al., 2013). This process is considerably more prominent in Janenschia than in any other sauropod, giving the astragalus an unusual outline in proximal view, and is thus regarded as an autapomorphy. The proximal surface of this posterior expansion is irregularly flat and faces dorsally and a little medially. In contrast, the proximal surface of the medial half of the astragalus is rugose but otherwise featureless. It slopes so that it faces dorsally, but also posteriorly. Medial to the posterior expansion, the astragalus tapers anteroposteriorly, although this reduction in length is entirely restricted to the posterior margin. The medial surface is rugose and faces medially and posteriorly.

No calcaneum is present (though see below), but it cannot be determined whether this element was genuinely absent, or merely not preserved. A calcaneum is present in most sauropods for which a well preserved lower hindlimb and metatarsus is known, but is lost in titanosaurs (McIntosh, 1990; Upchurch, 1995, 1998; Bonnan, 2000).

\section{Pes}

The right pes is nearly complete, with only the distal ends of the ungual claws of digits I-III missing (Figs 6-8; see Tables 2-3 for measurements). Metatarsal III is the longest, followed by metatarsals II, IV and I, with metatarsal V considerably shorter. The ratio of the longest metatarsal to tibia length is 0.2 , which is at the lower end of the spectrum of sauropods (Mannion et al., 2013). The phalangeal formula is 2-3-3-2-1 (Fraas, 1908), contrasting with the plesiomorphic condition in most non-neosauropods whereby three or more phalanges are typically present on digit IV (Upchurch, 1995, 1998; Nair \& Salisbury, 2012). Many of the phalanges are difficult to initially match up with the illustrations in Fraas (1908: p. 12), although this is because several are figured in unusual orientations.

The proximal end of metatarsal I has an approximately ' $D$ '-shaped outline, with a mildly concave lateral surface and a dorsolateral projection. In proximal end view the ventral and medial surfaces curve into one another, whereas the dorsal margin slopes so that it faces dorsally and medially. The proximal articular surface is irregular, becoming very mildly concave centrally. The lateral surface of the proximal half of the metatarsal is concave, whereas the remainder of this surface, as well as the entirety of the medial surface, is mostly flat. The ventral surface is transversely convex and anteroposteriorly concave along the length of the metatarsal. The region where the dorsal and distal surfaces meet is slightly damaged, but there is no evidence for a rugosity on the dorsolateral margin of the distal end, such as those seen in the first three metatarsals of diplodocids (Upchurch, 1995). The lateral distal condyle projects further distally than the medial one and is also dorsoventrally larger. There is no ventrolateral projection at the distal end. The distal articular surface is 
strongly convex dorsoventrally, extending onto the dorsal and ventral surfaces, and saddleshaped transversely, i.e. it is convex along the lateral and medial thirds, and concave centrally. A subtle ventral groove separates the two distal condyles.

Metatarsal II has a sub-rhomboidal outline in proximal end view, with the flat lateral and medial margins longer than the dorsal and ventral ones. The proximal articular surface is irregular, becoming convex towards the margins. Although slightly damaged, there is a ventromedial projection at the proximal end for articulation with metatarsal I. There is no evidence for a dorsolateral rugosity, but a ventrolateral projection is present near the distal end, with the area above this shallowly concave. A similar projection is present in diplodocids (Tschopp et al., 2015a), as well as some other taxa, including Bellusaurus (Mo, 2013: pl. 64). The lateral distal condyle projects a little further distally than the medial one, but the latter is slightly larger dorsoventrally. The distal articular surface is similar to that of metatarsal I, although the condyles are more clearly defined by a distinct ventral groove. The extension of the distal articular surface onto the dorsal surface of the metatarsal is strongly biased medially, although this is not as proximally extensive as in Alamosaurus (D'Emic, Wilson \& Williamson, 2011) or Euhelopus (Mannion et al., 2013).

In proximal end view, metatarsal III has a similar outline to metatarsal II, although the rhomboidal shape is a little more pronounced. The proximal articular surface is mildly convex towards the margins, lacking the domed surface of some sauropods (D'Emic et al., 2011). The lateral surface of the proximal quarter of the metatarsal is dorsoventrally concave, and a subcircular fossa is present at the distal end, both for reception of metatarsal IV. The distal condyles project approximately the same distance distally and are subequal in dorsoventral height. The distal articular surface is dorsoventrally convex, as in metatarsals I and II, but differs in that it is also transversely convex.

Metatarsal IV has a dorsoventrally elongate, suboval outline in proximal end view, and the proximal articular surface is mildly convex. The medial margin of the proximal end lacks the concavity for reception of metatarsal III that characterises many titanosauriforms (D’Emic et al., 2011; D’Emic, 2012; Mannion et al., 2013), although damage to this margin gives the impression that a concavity is present in some views. The medial surface of the distal end is dorsoventrally concave, but does not form the distinct fossa seen in metatarsal IIII. A short ridge on the ventrolateral margin bounds this concavity, but this feature is extremely subtle and could be a preservational artefact. The distal articular surface is strongly convex dorsoventrally and gently convex transversely, lacking any distinct bevelling. Although the dorsal surface of the distal end is damaged, the distal articular surface clearly extended strongly onto the dorsal surface of the metatarsal. There is no clear differentiation of the distal end into two distinct condyles, although this might partly be a result of abrasion of the ventral margin.

The proximal end of metatarsal $\mathrm{V}$ is subtriangular, with a flat ventral margin, and domed dorsal margin. As such, it contrasts with the dorsoventrally compressed proximal end of metatarsal $V$ seen in many somphospondylans (Poropat et al., 2016), e.g. Neuquensaurus (MLP MLP CS 1180: PDM \& PU pers. obs. 2013), Saltasaurus (PVL 4017-121: PDM \& PU pers. obs. 2013) and Tastavinsaurus (MPZ 99/9: PDM \& PU pers. obs. 2009). Laterally, the proximal articular surface is transversely convex, whereas the medial half is transversely concave. Although the metatarsal is funnel-shaped in dorsal view, the distal end is quite strongly expanded transversely. The distal articular surface is strongly convex in all directions and is not divided into distinct condyles. No tubercle is present on the ventral surface, contrasting with the condition in several titanosaurs, e.g. Epachthosaurus and Saltasaurus (Poropat et al., 2016). As noted above, metatarsal V is extremely short proximodistally, such 
that it is considerably shorter than metatarsal I (metatarsal I to $\mathrm{V}$ ratio of 1.34 ), a feature that Upchurch et al. (2004) listed as an autapomorphy of Janenschia. Although a shortened metatarsal V is widespread (Mannion et al., 2013), the extent of its reduction relative to metatarsal I in Janenschia is much greater than in other sauropods (note that the ratio provided by Mannion et al. [2013] for Diplodocus [1.31] was based on incorrect measurements in Hatcher [1901], and should instead be 0.76 [CM 89: PDM pers. obs. 2013]). As such, we regard this shortening of metatarsal $V$ as autapomorphic for Janenschia.

Phalanx I-1 is wedge-shaped in dorsal view, with a proximodistally longer medial than lateral margin. It lacks the proximoventral projection present in some diplodocids (Upchurch et al., 2004). The proximal articular surface is mediolaterally convex and dorsoventrally concave, whereas the distal articular surface is concave mediolaterally and strongly convex dorsoventrally. There is a dorsomedial projection at the distal end. The ventral surface is concave and a prominent ventral groove separates the two distal condyles, with the dorsoventrally larger medial condyle projecting further distally than the lateral one.

Phalanx II-1 is missing its lateral surface, but is otherwise complete. It is a larger and more robust element than $\mathrm{I}-1$, but has a similar morphology. The presence of the largest proximal phalanx occurring on digit II characterises most sauropods (Upchurch et al., 2004), e.g. Apatosaurus (Gilmore, 1936) and Turiasaurus (CPT-1195-1210: PDM \& PU pers. obs. 2009).

Although affected by crushing, phalanx II-2 is strongly compressed proximodistally, comparable to the condition in diplodocids (McIntosh, Coombs \& Russell, 1992) and Turiasaurus (CPT-1195-1210: PDM \& PU pers. obs. 2009). It is slightly wider mediolaterally than it is dorsoventrally, and decreases in proximodistal length towards its lateral margin. The distal articular surface is dorsoventrally convex and becomes transversely concave towards its centre.

In proximal view, phalanx III-1 is D-shaped, with a flat ventral margin. This cross section is largely maintained throughout the length of the element, and there is no notable decrease in dorsoventral height distally. The phalanx is longest along its medial margin, although the distal end has been crushed such that there is a prominent distomedial flange-like projection.

Phalanx III-2 is a greatly reduced, wedge-shaped element, which is difficult to orientate with confidence (its morphology, in articulation, with the remainder of the pes, is also difficult to discern from photographs presented by Fraas [1908: pl. 12]). As interpreted here, it decreases in dorsoventral height and proximodistal length towards its lateral margin, and has a dorsoventrally convex distal articular surface.

Phalanx IV-1 appears to have been orientated incorrectly by Fraas (1908), with the distal end interpreted as the proximal articular surface. As interpreted here, the phalanx is missing material from the dorsomedial corner of the proximal half. Although it might be slightly worn, the proximal articular surface is flat. The phalanx has a slightly ovoid cross section, with its long axis orientated mediolaterally, and a dorsoventrally taller medial third. In dorsal view, the medial and lateral margins are concave, as a result of the phalanx expanding transversely at its distal end. There is little in the way of bevelling of the distal end, and the distal articular surface is dorsoventrally convex.

Ungual claws are present on digits I-III, although in each case the distal end is restored. All three are recurved in lateral view and would have been strongly laterally deflected when articulated with their respective proximal phalanges. A groove is present at approximately midheight on the medial surface of each ungual, and follows the curvature of the element. The first two ungual claws are especially large, with I-2 (and probably II-3) 
considerably longer than its associated metatarsal. There is no evidence for the ventral tuberosity seen in the pedal unguals of many titanosauriforms (Canudo et al., 2008; Mannion et al., 2013).

We tentatively follow the identifications of Fraas (1908) for terminal phalanges IV-2 and V-1, although we note the possibility that each might belong on the other digit (J. Nair pers. comm., 2014). Phalanx IV-2 is a globular ungual that is difficult to orient with confidence. Based on what appears to be a flat, facet-like area that we interpret to be part of the proximal articular surface, the phalanx is interreted as being dorsoventrally compressed and mediolaterally elongate, with a gently convex dorsal surface and a more strongly convex ventral surface. Given its slightly unusual morphology, one possibility is that IV-2 actually represents the missing calcaneum, but we cautiously retain it as a pedal phalanx. Lastly, phalanx $\mathrm{V}-1$ is a reduced, wedge-shaped element that decreases in dorsoventral height and proximodistal length laterally. In proximal view, phalanx V-1 has a Dshaped outline, with a flat ventral margin. Its proximal articular surface is flat to irregularly concave, whilst the dorsal and distal surfaces merge smoothly into one another.

\section{DESCRIPTION AND COMPARISONS OF TOPOTYPIC MATERIAL OF JANENSCHIA ROBUSTA}

Pubis

The left pubis (MB.R.2090.2 [B8]) is missing most of its posterior margin, except for part of the distal end of the ischiadic articulation, and the distal end of the pubis blade (Fig. 9; see Table 4 for measurements). The iliac articulation has an anteroposteriorly elongate elliptical outline in proximal view, but does not form the extremely transversely compressed structure seen in some titanosaurs (Mannion \& Calvo, 2011). Only a small portion of the acetabular region is preserved. A well-developed ambiens process is present on the anterior surface of the pubis, close to the proximal end, although this does not form the 'hook'-shape seen in flagellicaudatans (McIntosh, 1990; Upchurch, 1998). Although this process tends to be greatly reduced and largely confluent with the anterior margin of the pubis in other sauropods (Mclntosh, 1990; Upchurch, 1998), the pubes of at least two additional nonflagellicaudatans also possess enlarged ambiens processes, i.e. Giraffatitan (Janensch, 1961; D'Emic, 2012) and Bellusaurus (Dong, 1990: pl. 3). Only the anterior margin of the obturator foramen is preserved in MB.R.2090.2, but it appears to have been a proximodistally elongate, elliptical opening. The ratio of the dorsoventral height of the ischiadic articulation to pubis length can be approximated as 0.44 , although this ratio is greatly variable among eusauropods (Mannion et al., 2013). There is no proximodistally elongate ridge along the lateral surface of the distal blade, differing from the condition seen in many derived titanosaurs (Powell, 2003; Salgado \& Carvalho, 2008; Otero, 2010; Poropat et al., 2016). The distal end does not form an anterior boot, in contrast to some titanosauriforms (Canudo et al., 2008; Mannion et al., 2013), but does expand prominently mediolaterally, differing from the laminar pubes of most somphospondylans (Curry Rogers, 2005; Poropat et al., 2016), e.g. Saltasaurus (PVL 4017-95: PDM pers. obs. 2013). The distal surface is convex in all directions, and a large, flat, triangular surface is present on the posteromedial surface of the distal end, which would have articulated with the comparable region of the right pubis.

\section{Ischium}

Two right ischia are preserved (Fig. 9; see Table 4 for measurements). MB.R.2090.4 (B13) is mostly complete except for some damage to the ventral margin of the blade and the base of the pubic articulation. The articular surface of the iliac peduncle is subcircular, 
narrowing slightly anteroposteriorly towards its lateral margin. Unlike many rebbachisaurids (Sereno et al., 2007), there is no constriction of the iliac peduncle at its base, and it does not form the large contribution to the acetabulum seen in brachiosaurids (D'Emic, 2012). The acetabular region narrows mediolaterally away from the iliac peduncle, and lacks the anterodorsal projection that characterises many titanosaurs (D'Emic, 2012; Mannion et al., 2013). It is not possible to determine whether there was any emargination distal to the pubic articulation. A low, rugose ridge, for attachment of $M$. flexor tibialis internus III (BorsukBiałynicka, 1977), is situated close to the posterodorsal margin of the lateral surface, at the base of the proximal plate. In contrast to more basal sauropods (Yates, 2007; D'Emic, 2012; Poropat et al., 2016), no groove is associated with this ridge. The dorsal margin of the ischial blade is mediolaterally thicker than that of the ventral margin and, although incomplete distally, the blade clearly expands dorsoventrally towards its distal end. In articulation, the distal ends of the conjoined ischia would have formed a ' $V$ '-shaped angle, rather than the coplanar morphology present in rebbachisaurids and most macronarians (Upchurch, 1998; Wilson \& Sereno, 1998).

A second, larger, right ischium (MB.R.2090.3 [B11]) is missing a large amount of material from the proximal end and along the blade margins. Its incomplete nature makes it difficult to determine much about its anatomy or whether or not it can be confidently referred to Janenschia, but it indicates the presence of a second individual amongst the holotypic and topotypic elements comprising Janenschia. As in MB.R.2090.4, the lateral ridge is low and is not associated with a groove. It is not possible to determine which ischium belongs to the same individual as the pubis, but the ischium to pubis length ratio is between 0.96 and 1.03 (see Table 4), similar to most sauropods, with the exception of some somphospondylans where this ratio is greatly reduced (Salgado et al., 1997; Mannion et al., 2013).

\section{Tibia}

The distal half and a separate portion of the shaft of a left tibia (MB.R.2090.1 [B6]) is preserved, and has similar proportions to the holotype right tibia (SMNS 12144) (see Tables 1 and 4), supporting the view that they might be from the same individual. As in most sauropods, the anterior surface of the distal end is flat. The posterior and lateral surfaces curve smoothly into one another, whereas the change of slope between the posterior and medial surfaces is more pronounced. The medial malleolus does not project as far laterally as the lateral malleolus, but extends further distally. As is the case in the holotype tibia, there is no groove along the posterior surface of the distal end.

\section{DESCRIPTION AND COMPARISONS OF MATERIAL REFERABLE TO JANENSCHIA ROBUSTA FROM QUARRY P}

Quarry P (Fig. 1) yielded a rich accumulation of material that appears to represent a single taxon (Heinrich, 1999b; Bonaparte et al., 2000; this study), and which can be referred to Janenschia based on a shared autapomorphy of the astragalus (see also Janensch, 1922, 1929a; Bonaparte et al., 2000), of which two are present and closely associated with other elements. Furthermore, no notable anatomical differences are present in any of the other overlapping elements. Four partial and articulated skeletons were recovered from the quarry and have been referred to as skeletons I-IV (Heinrich, 1999b; Bonaparte et al., 2000) (Fig. 10). Skeletons I and II comprised near complete left hindlimbs, whereas skeletons III and IV consisted of nearly complete left forelimbs, lacking humeri. Skeletons I and IV, and II and III, 
were found closely associated with one another, respectively (Heinrich, 1999b; Bonaparte et al., 2000), and seem to be from very similar sized individuals. Several additional elements were found scattered across the quarry: (1) a left humerus was found close to skeleton II; (2) pelvic, sacral and other vertebral elements, as well as a femur, were found adjacent to skeletons II and III; and (3) vertebral remains were found approximately $1 \mathrm{~m}$ away from skeletons I and IV. At least two of these vertebral remains were noted by Janensch as representing caudal vertebrae (Bonaparte et al., 2000). Unfortunately, many of these elements, listed and figured in Janensch's notes and field sketch of Quarry P (Heinrich, 1999b), have been subsequently lost or destroyed (although some might be in other German museums: OWM Rauhut, pers. comm. 2018), and were never previously described or figured (Bonaparte et al., 2000). Of this original wealth of material, all that remains is: (1) the humerus found near skeleton II; (2) the radius, ulna and one manual phalanx from skeleton III; (3) the radius, ulna, carpal and manual ungual claw from skeleton IV; (4) a femur which, based on its size, is presumably the element from skeleton I, rather than that found near skeleton III (although it is labelled as P22 rather than P1 as in the original quarry map; also see 'Indeterminate Tendaguru material previously referred to Janenschia: Quarry Nr. 22'); and (5) the tibiae, fibulae and astragali from skeletons I and II. A quarry map exists (Fig. 10), as well as additional close-up drawings of the pedal remains (see also Heinrich, 1999b; Bonaparte et al., 2000), but it is not possible to make detailed comparisons with the holotypic pes. Below, we describe the remaining material from Quarry $P$.

\section{Humerus}

The left humerus (MB.R.2095.7 [P8]) is mostly complete, although a little worn around its edges, particularly at the proximomedial corner (Fig. 11; see Table 5 for measurements). It is also missing a small amount of material from the lateral margin of the midshaft. In anterior view, the lateral half of the proximal end slopes downwards; thus, the humerus lacks the squared proximolateral corner that characterises somphospondylans (Upchurch, 1999; Wilson, 2002; Mannion et al., 2013), as well as Haestasaurus (Upchurch, Mannion \& Taylor, 2015) and Tehuelchesaurus (Carballido et al., 2011). There is expansion along the lateral, as well as medial margins of the proximal end, giving the humerus an hourglass outline in anterior view, rather than the asymmetrical, laterally unexpanded morphology present in most titanosauriforms and several turiasaurs (Tschopp et al., 2015a; Poropat et al., 2016; Mannion et al., 2017). The proximal end is anteroposteriorly thickest towards its medial edge, with the humeral head forming a prominent rounded bulge at the proximal tip of the posterior surface. A similar posterior process is also present in Giraffatitan, Haestasaurus and Ligabuesaurus (Bonaparte et al., 2006; Upchurch et al., 2015). Although probably accentuated by wear, the proximomedial corner is strongly rounded, and lacks the sinuous outline that characterises some titanosaurs (Upchurch, 1998; González Riga, 2003).

The deltopectoral crest has been heavily worn, but clearly projected anteriorly or slightly anterolaterally, and does not become transversely expanded distally, or project medially, contrasting with many titanosauriforms (Wilson, 2002; Mannion et al., 2013; Poropat et al., 2016). Medial to the deltopectoral crest, the anterior surface of the proximal half of the humerus is mildly concave mediolaterally and there is evidence for a centrally positioned, low, rounded muscle scar for attachment of $M$. coracobrachialis. There are no lateral excavations or ridges on the deltopectoral crest, and the humerus lacks the posterolateral bulges for M. scapulohumeralis anterior and for M. latissimus dorsi that are present in several derived titanosaurs (Borsuk-Białynicka, 1977; Otero, 2010; D'Emic, 2012; Upchurch et al., 2015). The proximal half of the posterior surface is mediolaterally convex. 
There is little in the way of torsion between the proximal and distal halves of the humerus, in contrast to the strong degree of twisting seen in diplodocids (Tschopp et al., 2015a). In anterior view, the medial and lateral margins of the shaft are concave. At midshaft, the humerus is not strongly compressed anteroposteriorly, and it remains mediolaterally wide relative to the humeral length (ratio $=0.16$ ), in contrast to the gracile humeri of brachiosaurids and some other taxa (e.g. Ligabuesaurus) (Curry Rogers, 2005; Mannion et al., 2013). Distally, the mediolateral width of the humerus is strongly expanded relative to the proximodistal humeral length, with a ratio of 0.36 . With the exception of apatosaurines and some derived titanosaurs (Poropat et al., 2016), this ratio is much higher than in other eusauropods, with only Haestasaurus (0.35) otherwise possessing a comparable ratio (Upchurch et al., 2015). The distal half of the anterior surface is mildly convex mediolaterally, becoming more strongly convex towards the very distal end. The lateral half of the anterior distal surface has a divided condyle, differing from the condition seen in most derived somphospondylans (D'Emic, 2012; Mannion et al., 2013). Medial to this condyle, the anterior surface is concave, and the distomedial corner is slightly expanded anteriorly. The anterior surface, lateral to the condyle is mediolaterally concave, with the humerus thinning anteroposteriorly. The lateral surface of the distal end forms two distinct faces. The anterior half is formed by the anterolaterally facing, mediolaterally concave surface described above, whereas the mildly anteroposteriorly convex posterior half faces laterally. The medial surface of the distal end is relatively flat, and faces medially and slightly posteriorly. Although slightly damaged, the posterior surface of the distal end is mainly flat, with only a very mildly concave anconeal (= supracondylar) fossa. In this regard, it differs from the ridge-bound, deep fossa of the humeri of most somphospondylans and Giraffatitan (Upchurch et al., 2004; Mannion \& Calvo, 2011; Mannion et al., 2013; Upchurch et al., 2015). The undivided distal articular surface is rugose and anteroposteriorly convex.

\section{Radius}

The left radius (MB.R.2095.9 [P11]) is largely complete (Fig. 12; see Table 5 for measurements), but has been broken into numerous pieces and re-assembled; thus it is difficult to know whether the length is entirely reliable. The outer bone is also missing at several points throughout the element, particularly along the shaft. In dorsal view, the mildly concave proximal end narrows slightly anteroposteriorly towards its medial margin to form a gentle medial projection. The proximal end is not strongly expanded relative to the length of the radius, and is mediolaterally narrower than the distal end, as is also the case in Haestasaurus (Upchurch et al., 2015), Tehuelchesaurus (Carballido et al., 2011), and some derived titanosaurs (Curry Rogers, 2005). Conversely, the proximal end is anteroposteriorly wider than the distal end, contrasting with the unusual morphology observed in the contemporaneous turiasaurs Turiasaurus and Zby (Mateus, Mannion \& Upchurch, 2014). Unlike Giraffatitan, Haestasaurus, and some titanosaurs (Upchurch et al., 2015), there is no ridge on the medial margin of the proximal end, for attachment of $M$. biceps brachii and $M$. brachialis inferior (Borsuk-Białynicka, 1977).

There is no notable twist in the axis between the proximal and distal halves of the radius, unlike the stongly twisted conditions seen in several somphospondylans, such as Epachthosaurus (UNPSJB-PV 920: PDM \& PU pers. obs. 2013) and Huabeisaurus (D'Emic et al., 2013). Although the posterior surface of the distal end is very poorly preserved, there are clearly ridges present, but these do not extend far proximally, in contrast to Aragosaurus (Royo-Torres et al., 2014) and many titanosaurs (Curry Rogers, 2005). The lateral surface of the distal end is mildly convex anteroposteriorly, whereas the medial surface is more 
strongly convex. In anterior view, the lateral two-thirds of the distal end are strongly bevelled, sloping at an angle of approximately $20^{\circ}$ to the horizontal. Well-developed distal bevelling of the radius characterises many titanosaurs (Wilson, 2002), but is also present in a number of basal macronarians, Apatosaurus, and several non-neosauropods (Mannion et al., 2013; Mateus et al., 2014; Upchurch et al., 2015; Poropat et al., 2016). The distal end is transversely expanded relative to the shaft (ratio $>2.0$ ), comparable to many macronarians (Wilson, 2002; Mannion et al., 2013), as well as Zby (Mateus et al., 2014). The posterior margin of the distal end is gently excavated, as is the case in many neosauropods (Upchurch et al., 2015), as well as Bellusaurus (Mo, 2013).

A second radius (MB.R.2095 [P10]; see Table 5 for measurements) is poorly preserved and missing much of the margins from its proximal and distal ends; as such, no further anatomical information on the morphology of the radius can be gleaned. Both radii are the same length, and the radius to humerus (MB.R.2095.7) length ratio is 0.67.

Ulna

The proximal two-thirds and distal end of a left ulna (MB.R.2095.8 [P9]) are preserved, but the shaft in between is heavily restored and the anterolateral process is incomplete. A second left ulna (MB.R.2095.11 [P12]) is slightly more complete, although the distal tip of the anterolateral process is missing. The two ulnae do not differ significantly from one another and are therefore described together (Fig. 13; see Table 5 for measurements). The ulna is robust, with a proximal end mediolateral width to ulna length ratio of 0.47 , comparable to many derived somphospondylans (Wilson, 2002; Curry Rogers, 2005; Mannion et al., 2013), as well as Haestasaurus (Upchurch et al., 2015) and Bellusaurus (Mo, 2013). A prominent olecranon process is present, projecting well above the proximal articulation. Within Sauropoda, a well-developed olecranon process is otherwise known primarily in derived titanosaurs (Wilson \& Sereno, 1998; Mannion et al., 2013), but is also present in Haestasaurus (Upchurch et al., 2015) and some mamenchisaurids (Sekiya, 2011).

In proximal view, the angle between the long-axes of the anteromedial and anterolateral processes is acute $\left(<80^{\circ}\right)$. The anteromedial proximal process has a concave profile in anterior view, a feature that characterises a number of titanosauriforms (Upchurch, 1995, 1998; D'Emic, 2012; Mannion et al., 2013), as well as Haestasaurus (Upchurch et al., 2015). Although the anterolateral proximal process is incomplete, it was clearly not significantly shorter than the anteromedial process, contrasting with the condition in a number of sauropods (especially several brachiosaurids) whereby the anteromedial process is much longer (Wilson, 2002; Mannion et al., 2013; Upchurch et al., 2015). In between the two proximal processes, the anterior surface of the proximal end is mediolaterally concave, although this region is poorly preserved. The posterior process of the proximal end is only weakly developed, in contrast to the prominent structure developed in several derived titanosaurs (Upchurch et al., 2015). The posterior surface of the proximal end is mediolaterally concave, mainly as a result of the distal extension (to approximately midlength) of the posterior process as a strongly rounded ridge.

There is no ridge and groove structure along the posterolateral surface of the distal half of the ulna, contrasting with the condition observed in the turiasaurs Losillasaurus and Turiasaurus (Royo-Torres et al., 2006). At its distal end, the ulna is anteroposteriorly expanded. In distal end view, the ulna has a D-shaped outline, with the long axis oriented anteroposteriorly, and a gently excavated medial margin and convex lateral margin. The rugose distal articular surface is mildly convex mediolaterally and more strongly convex anteroposteriorly. 


\section{Carpometacarpus}

The left carpal (MB.R.2095.10 [P11]) is fairly complete (Fig. 14; see Table 5 for measurements), although poorly preserved in places. Its mediolateral diameter is much greater than its anteroposterior diameter (ratio=1.5; Table 5), similar to that of the carpals of Apatosaurus (ratio=1.4; Gilmore, 1936: fig. 14) and Mamenchisaurus (ratio=1.5; Ouyang \& Ye, 2002), but contrasting with the equidimensional carpals in the turiasaurs Losillasaurus (ratio=1.2; MCNV Lo: PDM \& PU pers. obs. 2009) and Turiasaurus (ratio=1.0; CPT-1195-1210: PDM \& PU pers. obs. 2009), and the basal macronarians Aragosaurus (ratio=1.1; Royo-Torres et al., 2014), Camarasaurus (ratio=1.1; Mclntosh et al., 1996: table 12), Lourinhasaurus (ratio=1.1; Mocho, Royo-Torres \& Ortega, 2014) and Vouivria (ratio=1.1; Mannion et al., 2017).

The Janenschia carpal increases in proximodistal thickness towards its posterior and lateral margins. The anterior and lateral margins meet at an angle close to $90^{\circ}$, whereas the posterior and medial margins curve into one another, giving the carpal a quadrant shape in proximal view. The proximal surface is fairly flat, whereas the distal surface is more irregular as a result of the posterolateral thickening. The distal surface lacks the well-developed medial distal process present in the carpal of Turiasaurus (Royo-Torres et al., 2006), with only a shallow concavity separating the medial and lateral halves of this surface. Several shallow, subcircular fossae are present on the posterior surface.

The left ungual manual claw (MB.R.2095.13 [P32]) is missing its distal tip and small amounts of material from its margins (Fig. 14; Table 5). It curves laterally and is not strongly compressed mediolaterally. The proximal articular surface is mildly concave. In lateral view, the ungual decreases in height along its dorsal margin. The ventral surface is poorly preserved, but is clearly not strongly arched. The medial surface is dorsoventrally convex, whereas the poorly preserved lateral surface is only very mildly convex. Small vascular foramina are present along the medial and lateral surfaces.

A poorly preserved manual phalanx (MB.R.2245 [P54]) might represent IV-1 or V-1 (Fig. 14; Table 5). Its proximal articular surface is flat to mildly concave and has an elliptical outline. The phalanx decreases in mediolateral width and dorsoventral height (mainly along the ventral margin) distally. The distal articular surface is strongly convex and lacks distinct condyles.

\section{Femur}

The left femur (MB.R.2707 [P22]) is broken into two pieces, although it appears to be largely complete (Fig. 15; see Table 6 for measurements). The femoral head projects dorsomedially. Above the weakly developed lateral bulge, the proximolateral margin is medially deflected, as is the case in most basal macronarians (Mannion et al., 2013), but also several non-neosauropod taxa, such as Jobaria (MNN specimens: PDM pers. obs. 2012). No trochanteric shelf or associated ridge is present on the posterior surface, contrasting with the condition in a number of somphospondylans and rebbachisaurids (Sereno et al., 2007; Otero, 2010; Whitlock, 2011a; Mannion et al., 2013). The anterior surface of the femur is mainly flat and featureless, lacking the proximodistally elongate ridge (linea intermuscularis cranialis) that characterises several derived titanosaurs (Otero, 2010; D'Emic, 2012).

The fourth trochanter is situated close to the medial margin of the posterior surface and is slightly biased towards the proximal half of the femur. Unlike several macronarians, e.g. Camarasaurus and Giraffatitan (Whitlock, 2011a; Mannion et al., 2013), it is not visible in anterior view. The midshaft is anteroposteriorly compressed (mediolateral width to 
anteroposterior width ratio $=1.72)$, but this is not as extreme as the condition in many titanosauriforms (Wilson, 2002; D'Emic, 2012; Mannion et al., 2013).

In anterior view, the distal end of the femur is near-perpendicular to the long axis of the femoral shaft, lacking the bevelled morphology of some sauropods (Wilson, 2002; Mannion et al., 2013). The transverse expansion of the distal end is mainly restricted to the medial margin. As well as facing medially, the flat medial surface of the distal end is deflected to face slightly anteriorly and dorsally. The lateral surface of the distal end is flat along its anterior half, but becomes anteroposteriorly convex posteriorly. The posterior surface of the fibular condyle is divided, but the surface has been partly worn away. A deep concavity separates the subequal tibial and fibular distal condyles on the posterior surface. The distal articular surface is strongly convex anteroposteriorly, extending onto the posterior surface, but only very slightly onto the anterior surface of the femur. The middle third of the distal surface is deflected to face anteriorly, although this deflection is not as prominent as in the holotype femur of Janenschia (SMNS 12144).

Tibia

Two left tibiae are preserved (MB.R.2095.1 [P2] and MB.R.2095.4 [P5]): both are largely complete in terms of length (Fig. 16; see Table 6 for measurements), but are missing most or all of their cnemial crests, and the distal condyles are incomplete. MB.R.2095.4 is the better preserved of the two tibiae and shows the presence of a well developed 'second cnemial crest', separated from the true cnemial crest by a notch. Unfortunately, the laterally projecting cnemial crest is too incomplete to determine whether the prominent tubercle observed on the holotype tibia of Janenschia (SMNS 12144) is present. As is the case in the holotypic and topotypic tibiae, no groove is present along the posterior surface of the distal end.

Fibula

The left fibula (MB.R.2095.2 [P3]) is mostly complete (Fig. 17; see Table 6 for measurements) but has been broken in numerous places and re-assembled. The margins of the shaft have also been worn away, as has much of the outer bone surface. The proximal end is mediolaterally compressed and anteroposteriorly expanded, particularly along the posterior margin. The proximal articular surface is convex and there is no proximal anteromedial crest. The medial surface of the proximal end is mildly concave anteroposteriorly, whereas the lateral surface is gently convex. However, preservation is too poor to determine whether a striated triangular area is present medially. The lateral muscle scar is also not preserved. The distal end seems to expand both anteroposteriorly and mediolaterally relative to the shaft, and the distal articular surface is convex. A second left fibula (MB.R.2095.5 [P6]) is very poorly preserved and missing its distal end and portions of the shaft (Fig. 17; see Table 6 for measurements). The medial surface of the proximal end displays some evidence for the presence of striations. Otherwise, where anatomical information can be gleaned, it does not provide additional information or differ from MB.R.2095.2.

\section{Astragalus}

Two left astragali are preserved (MB.R.2095.3 [P4] and MB.R.2095.6 [P7]; Fig. 18; see Table 6 for measurements). Both are complete, although MB.R.2095.3 is poorly preserved in some regions, and the posterior projection has been broken off and re-attached, with some material lost in this process. The two astragali do not differ in any notable way and therefore 
are described together. The astragalus decreases in proximodistal height and anteroposterior length towards its medial margin. The majority of this shortening occurs along the posterior margin. Its distal surface is anteroposteriorly convex, and gently convex mediolaterally. The ascending process extends to the posterior margin of the astragalus, and is subrectangular in proximal view, tapering anteroposteriorly towards its medial margin. The lateral surface of the ascending process is mildly concave anteroposteriorly and flat to mildly concave proximodistally, although it becomes strongly concave dorsoventrally towards its dorsal margin. Two large, subcircular fossae are present on the upper half of the lateral surface, with a smaller, shallower fossa in between (this region is too poorly preserved in MB.R.2095.3 to ascertain whether such fossae are present). No distolateral lip is present. The posterior margin of the lateral half of the astragalus is concave in proximal view, as a result of an extremely prominent posterior process, situated at approximately the midpoint of the posterior margin. In this regard, the two astragali share the autapomorphic morphology described in the holotype astragalus of Janenschia (SMNS 12144). A ridge extends posteromedially from the ascending process. Anterior and posterior to this ridge, a large, circular fossa opens dorsally (this region is too poorly preserved in MB.R.2095.3 to ascertain whether such fossae are present). The remaining proximal surface, along the medial third of the astragalus, is flat and featureless, and faces posterodorsally.

SAUROPODA MARSH, 1878

EUSAUROPODA UPCHURCH, 1995

TURIASAURIA ROYO-TORRES, COBOS \& ALCALÁ, 2006

TENDAGURIA BONAPARTE, HEINRICH \& WILD, 2000

Type species: Tendaguria tanzaniensis Bonaparte, Heinrich \& Wild, 2000

Gigantosaurus robustus Janensch, 1929a

Holotype: MB.R.2092.1-2 (NB4 and 5) - two associated anterior dorsal vertebrae.

Type locality and horizon: Nambango (site NB), about $15 \mathrm{~km}$ southeast of Tendaguru Hill, Lindi District, southeastern Tanzania (Fig. 1); probably from the Upper Dinosaur Member (Upper Saurian Bed), Tendaguru Formation; Tithonian, Late Jurassic (Janensch, 1929a; Bonaparte et al., 2000; Aberhan et al., 2002; Bussert et al., 2009).

Revised diagnosis: Tendaguria can be diagnosed by one autapomorphy (marked with an asterisk), as well as one local autapomorphy: (1) dorsal surface of anterior dorsal vertebral diapophyses excavated by two fossae (one posterolateral to the prezygapophyses, along the anterior half of the diapophysis, and one posterior to the prezygapophyses)*; and (2) prespinal midline ridge in anteriormost dorsal neural spines.

Additional comments: These dorsal vertebrae were originally referred to Janenschia robusta by Janensch (1929a), although no clear basis was given for this attribution. A sacrum and ilium were also found at this locality (Janensch, 1929a), but were never described or figured. These elements could not be located in the MfN collections and thus cannot be used to link Tendaguria to Janenschia because of a lack of overlapping material (see also Bonaparte et al., 2000). 
DESCRIPTION AND COMPARISONS OF TENDAGURIA TANZANIENSIS

Both dorsal vertebrae (Dv) are predominantly complete, but large portions are coated in plaster, obscuring the true morphology in places (Figs 19, 20). Based on the position of the parapophysis on the dorsolateral corner of the centrum (MB.R.2092.2) and on the centrum-arch junction (MB.R.2092.1), they are anterior dorsal vertebrae, probably representing Dv2 and Dv3 respectively (note that each of these specimen numbers pertained to the other vertebra in the description by Bonaparte et al. [2000]). Measurements are presented in Table 7. Nomenclature of vertebral laminae and pneumatic fossae follows Wilson $(1999,2012)$ and Wilson et al. (2011).

The opisthocoelous centrum is strongly compressed dorsoventrally, such that the posterior width to height ratio is greater than 1.5 (Table 7). This extreme compression is comparable to the condition in the anteriormost dorsal vertebrae of derived titanosaurs, such as Malawisaurus, Mendozasaurus, Opisthocoelicaudia and Rapetosaurus, the diplodocid Apatosaurus, and the turiasaurs Mierasaurus and Moabosaurus (Table 8; see also Mannion et al., 2013). The ventral surface of the centrum is convex mediolaterally, becoming flatter towards the midline. There are no ventral ridges or excavations. A moderately deep pneumatic foramen excavates the lateral surface of the centrum, although this does not ramify quite as far as the centrum midline. This foramen is divided by an anteroposteriorly thick, subvertical ridge, with the posterior half a little larger anteroposteriorly than the anterior half. The foramen is not acute posteriorly, contrasting with those in most macronarians (Upchurch, 1998); nor is it set within a fossa, differing from the condition in most titanosaurs (Bonaparte \& Coria, 1993; Upchurch et al., 2004), and some basal titanosauriform taxa (Mannion et al., 2013).

A steep, posterodorsally oriented paradiapophyseal lamina (PPDL) extends from the dorsal margin of the large, subcircular parapophysis up to the diapophysis. In Dv2, the long, anteroposteriorly thin posterior centrodiapophyseal lamina (PCDL) is oriented anterodorsally along its ventral portion, becoming mainly vertical dorsally, whereas it is oriented primarily vertically throughout its length in Dv3. In both vertebrae, the PPDL and PCDL merge at around the point where they begin to define the ventral margin of the diapophysis. A deep, triangular centrodiapophyseal fossa (CDF) is bounded by the PPDL anterodorsally, the PCDL posterodorsally, and the upper margin of the lateral pneumatic foramen ventrally. The latter 'boundary' could conceivably be classed as a thick posterior centroparapophyseal (PCPL) lamina, especially as it is directed anterodorsally at a shallow angle; however, it is too heavily coated in plaster to be certain of its identification.

Whereas the neural canal is circular at its posterior opening, it is semicircular at its anterior end, with a flat ventral margin. The undivided centroprezygapophyseal laminae (CPRLs) are mediolaterally wide walls of bone. Above the CPRLs, the prezygapophyses are mediolaterally elongate structures, with flat to mildly convex articular surfaces that face dorsally, as well as slightly medially. The two prezygapophyses are widely separated along the midline and connected by a dorsoventrally thick, anteroposteriorly short, horizontal interprezygapophyseal lamina (TPRL). Stout centropostzygapophyseal laminae (CPOLs) bound the lateral margins of the posterior opening of the neural canal, merging to form one sheet of bone (interpostzygapophyseal lamina [TPOL]) above. The postzygapophyses are mediolaterally wide structures, with flat to mildly concave articular surfaces that face ventrally and slightly laterally. As such, the zygapophyseal table is oriented at approximately $30^{\circ}$ to the horizontal, similar to the condition in most neosauropods and some taxa just outside of Neosauropoda (e.g. Jobaria and Mamenchisaurus), but contrasting with the 
subhorizontal plesiomorphic state (also present in brachiosaurids), as well as the steeply inclined zygapophyseal table of many titanosaurs and rebbachisaurids (Carballido et al., 2012). No hyposphene is present, in keeping with the view that these represent two of the anteriormost dorsal vertebrae.

Dorsoventrally tall epipophyses are present on the dorsal surfaces of the postzygapophyses; these do not extend beyond the posterior margin of the postzygapophyses. Whereas epipophyses are present in the cervical vertebrae of most sauropods (Yates, 2007; Wilson \& Upchurch, 2009; Mannion et al., 2013), their extension into anterior dorsal vertebrae is much less common, and they tend to be greatly reduced structures where present. However, Tendaguria shares the presence of prominent epipophyses with the turiasaur Moabosaurus (Britt et al., 2017).

The prezygodiapophyseal (PRDL) and postzygodiapophyseal laminae (PODL) are prominent, near-horizontal structures. The posterior surface of the neural arch, lateral to the $\mathrm{CPOL}$, is gently excavated, although this does not form a sharp-lipped fossa. Lateral to this excavation, in MB.R.2092.1, there is a stout, rounded, near-vertical ridge. This begins at approximately two-thirds of the way up the neural arch and merges with the ventral surface of the PODL, just medial to the lateral tip of the postzygapophysis. A moderately deep, triangular prezygapophyseal centrodiapophyseal fossa (PRCDF) is bounded anteriorly by the CPRL, posteroventrally by the PPDL, and dorsally by the PRDL. Within the PRCDF, close to its posterior margin, is a subvertical accessory lamina. The PRCDF also extends laterally onto the proximal half of the anterior surface of the diapophysis.

The diapophyses project laterally, curving downwards towards their distal tips. This is the case in the anterior dorsal vertebrae of most sauropods, with the exception of some somphospondylans (e.g. Euhelopus, Malawisaurus and Saltasaurus), dicraeosaurids and rebbachisaurids, in which the diapophyses project strongly dorsolaterally (Whitlock, 2011a; Mannion et al., 2013). The concave and rugose articular surface of each diapophysis (i.e. the lateral or distal surface) is subtriangular or wedge-shaped in lateral view, with the apex of this triangle pointing ventrally and slightly posteriorly. As well as the aforementioned PRCDF on the proximal half of the anterior surface, there is an additional excavation near to the lateral tip of the diapophysis that opens ventrally and anteriorly. This additional excavation is also present in Moabosaurus (Britt et al., 2017). There are two excavations along the dorsal surface of each diapophysis: one posterolateral to the prezygapophyses, along the anterior half of the diapophysis, and one posterior to the prezygapophyses. In both cases, these fossae are deeper in Dv2. These fossae are absent in Moabosaurus and are potentially diagnostic for Tendaguria. The posterior surface of the diapophysis is in general dorsoventrally concave, mainly as a result of the overhanging PODL. In Dv2, a dorsolaterally oriented ridge runs along the posterior surface at approximately midlength.

In Dv2, there are medial and lateral branches of the spinoprezygapophyseal lamina (SPRL), with a gentle fossa formed in between. The medial branch (mSPRL) extends from the posteromedial corner of the prezygapophyses posterodorsally, merging into the anterior surface of the neural spine at approximately midheight. The lateral branch of the SPRL (ISPRL) extends from near the posterolateral margin of the prezygapophysis and forms the anterolateral margin of the neural spine. It does not merge with the MSPRL. In Dv3, a poorly developed spinodiapophyseal lamina (SPDL) forms the anterolateral margin of the neural spine instead, and appears to be homologous to the ISPRL described in DV2. This is comparable to the example of 'lamina capture' highlighted by Wilson (2012) in the anterior dorsal vertebrae of the non-neosauropod eusauropod Jobaria, whereby the diapophysis migrates to a position close to the prezygapophysis and 'captures' its lamina. This transition 
can also be seen in other basal eusauropods, including Bellusaurus (Mo, 2013: pls. 24, 25 and 27) and Haplocanthosaurus priscus (Hatcher, 1903: pl. 1).

In both dorsal vertebrae of Tendaguria, a midline prespinal ridge is present, but only in Dv3 is there evidence for a weakly developed midline postspinal rugosity. The presence of a prespinal lamina in anteriormost dorsal vertebrae is a feature primarily restricted to titanosaurs (Salgado et al., 1997; D'Emic, 2012), but is also present in Haplocanthosaurus priscus (CM 572: PDM pers. obs. 2013). It is here regarded as a local autapomorphy of Tendaguria.

A subcircular fossa excavates the anterior surface of the neural spine, either side of the midline. It is defined ventromedially by the ISPRL in DV2, and by the SPDL in Dv3, in which this spinodiapophyseal fossa (SDF) deepens. A comparable SDF is present in the anteriormost dorsal vertebrae of Moabosaurus (Britt et al., 2017: figs 19, 20). In between the postzygapophyses, there is a deep, subcircular postspinal fossa. Spinopostzygapophyseal laminae (SPOLs) are oriented anteromedially and very slightly dorsally. The unbifurcated neural spine is an anteroposteriorly thin structure that is subrectangular in anterior view, with its long axis oriented mediolaterally. It is extremely short dorsoventrally, with the apex only slightly higher than the epipophyses. Such a low neural spine is unusual, and in nearly all other taxa with low anterior dorsal neural spines this structure is bifid (e.g. Euhelopus [Wilson \& Upchurch, 2009]). However, Tendaguria shares an extremely low, non-bifid anterior dorsal neural spine with Moabosaurus.

\section{INTERNAL TISSUE STRUCTURE OF TENDAGURIA TANZANIENSIS}

Both dorsal vertebrae are heavily composed of plaster, particularly in the centra and diapophyses; as such, many informative characters for vertebral pneumaticity are not preserved (Fig 21A-M). Each vertebra is internally stabilized by large metal rods, positioned at the level of the diapophyses. Although these rods are visible in the CT section (Fig. 21), they do not produce scattering effects. Where genuine bone is present, this is mostly compact ('massive') and apneumatic. The few pneumatic structures that are present are limited to rounded camerae surrounded by larger areas of massive bone (Fig 21).

The centrum of MB.R.2092.1 is nearly completely re-modelled with plaster, and the few patches of original bone are massive and apneumatic (Fig. 21B-E). The condyle of MB.R.2092.2 is excavated by a few large, pneumatic camerae, occupying the middle twothirds of the cross-section. These camerae are surrounded by massive bone, and are separated from each other by thin walls of bone (Fig. 21G-K). Posteriorly, these camerae develop into larger left and right camerae, before decreasing in size and becoming isolated from one another by massive walls of bone. They close directly anterior to the cotyle. A small, nearly circular camera is positioned dorsolateral to these main camerae (Fig. 21I). This smaller camera increases in size posteriorly, forming part of a system of three larger pneumatic camerae that hollow out the diapophysis (Fig. 211). Along the posterior part of the diapophysis, the dorsalmost of these camerae opens laterally into the CDF, whereas the medialmost of these camerae opens laterally into the pneumatic foramen on the lateral surface of the centrum (Fig. 21K-I). The diapophysis, better preserved in MB.R.2092.1, is hollowed out by a few small internal and rounded pneumatic camerae (Fig. 21B). The neural arch and spine consist entirely of compact, apneumatic bone.

Both dorsal vertebrae are pneumatic, with the pneumatic structures present being camerae of different sizes. This pneumatisation pattern can be described as camerate (Wedel, Cifelli \& Sanders, 2000; Wedel, 2003), and is restricted to the centrum and 
diapophyses. It most closely resembles the pattern of a dorsal vertebra of Diplodocus (Wedel, 2005: fig. 7.1h-j), and is substantially different to the camellate or somphospondylous 'honeycomb-shaped' pneumatisation pattern that characterises the presacral vertebrae of Galveosaurus + Titanosauriformes (Wedel, 2003, 2005; Carballido et al., 2011; Mannion et al., 2013).

\section{SAUROPODA MARSH, 1878 \\ EUSAUROPODA UPCHURCH, 1995 \\ TURIASAURIA ROYO-TORRES, COBOS \& ALCALÁ, 2006 \\ cf. Tendaguria}

Material: MB.R.2091.31 (G45) - middle-posterior cervical vertebra from Quarry G, located approximately $600 \mathrm{~m}$ south of Tendaguru Hill, Lindi District, southeastern Tanzania (Fig. 1); Upper Dinosaur Member (Upper Saurian Bed), Tendaguru Formation; Tithonian, Late Jurassic (Janensch, 1929a; Bonaparte et al., 2000; Aberhan et al., 2002; Remes, 2007; Bussert et al., 2009).

\section{DESCRIPTION AND COMPARISONS OF MB.R.2091.31}

Bonaparte et al. (2000) tentatively referred a cervical vertebra (MB.R.2091.31) to Tendaguria tanzaniensis, primarily on the basis of its low, undivided neural spine. MB.R.2091.31 is a vertebra from the middle to posterior region of the cervical series (see Table 7 for measurements). Overall it is fairly complete (Fig. 22), although it is missing some material from the right side of the neural arch, and has undergone some further damage in places since its description by Bonaparte et al. (2000) (see also Janensch, 1929a: fig. 9).

The centrum is anteroposteriorly short, with an average Elongation Index (aEI = centrum length [excluding condyle] divided by the mean average value of the posterior mediolateral width and dorsoventral height) of 1.5 . The ventral surface of the opisthocoelous centrum is extremely thin dorsoventrally. This surface is strongly concave mediolaterally along its anterior third (excluding the condyle), with this concavity decreasing in prominence posteriorly, such that the posteriormost portion of the ventral surface is flat. A sharp, anteroposteriorly oriented midline ridge runs along the deepest portion of this ventral concavity. The possession of a midline keel or ridge along the ventral surface of cervical centra is the plesiomorphic sauropod condition (Upchurch, 1998), but this is lost in many derived eusauropods (Poropat et al., 2016), with the notable exception of dicraeosaurids and rebbachisaurids (Whitlock, 2011a).

Although both parapophyses are incomplete, it is clear that their dorsal surfaces are unexcavated, as is also the case in non-neosauropods and many derived somphospondylans (Upchurch, 1998; Mannion et al., 2013). The parapophyses are restricted to the anterior third of the non-condylar centrum and project mainly laterally, contrasting with the strongly ventrolaterally projecting middle-posterior cervical parapophyses of several somphospondylans (the mid-Cretaceous East Asian taxa Daxiatitan, Erketu and Euhelopus [Wilson \& Upchurch, 2009; D'Emic, 2012], and the Late Cretaceous Argentinean titanosaur Overosaurus [Coria et al., 2013]), as well as the diplodocoids Apatosaurus and Nigersaurus (Mannion et al., 2013). The lateral surface of the centrum is heavily and deeply excavated, with the lateral pneumatic foramen divided into numerous separate chambers by subvertical laminae. This lateral foramen occupies most of the centrum length. Either a breakage or a 
possible further excavation within the foramen reveals additional pneumatic space further within the centrum.

The diapophysis is supported by a prominent PCDL that projects anterolaterally, as well as very slightly dorsally. There is no evidence for an anterior centrodiapophyseal lamina $(A C D L)$, but its absence might be a preservational artefact. At the anterolateral corner of the dorsal surface of the centrum, a stout ridge bifurcates into a prominent CPRL medially, and a second lamina laterally that runs mainly vertically, joining the medial-most portion of the PRDL. As this lamina does not meet the prezygapophysis, it is not an instance of a true bifid CPRL, a feature that appears to be restricted to diplodocoid cervical vertebrae (Whitlock, $2011 a, b)$. A deep subtriangular fossa is formed in between these two laminae. The PRDL is a dorsoventrally thin, plate-like structure that projects posterolaterally and slightly ventrally. The dorsal surface of the PRDL is excavated by a shallow, though extensive fossa. The prezygapophysis extends only a very short distance beyond the anterior condyle of the centrum. Its dorsomedially facing articular surface is mildly convex mediolaterally, whereas the articular surface of the postzygapophysis is mildly concave and faces ventrolaterally. The dorsal surface of the postzygapophysis is rugose and forms an epipophysis; the latter does not extend beyond the posterior margin of the postzygapophysis. There is no 'preepipophysis' or epipophyseal-prezygapophyseal lamina (EPRL). A prominent, dorsoventrally thin, posteroventrally oriented PODL is present.

Large portions of the outer surface of the neural arch have been broken away, presumably because the arch is highly pneumatic (see below). In dorsal view, the neural spine has a trapezoidal outline, with mildly concave anterior and posterior margins, and a mediolaterally oriented long-axis. In lateral view, the anterior margin of the neural spine slopes at an angle of approximately $60^{\circ}$, such that it faces anterodorsally, whereas the posterior margin is mainly vertically oriented. The anterior surface of the neural spine is rugose. There is evidence for a SPRL, a deep postspinal fossa, and a SPOL, although the latter is coated in plaster. The unbifurcated neural spine is extremely low, projecting only a short distance above the level of the postzygapophyses. Although low middle-posterior cervical neural spines are known in other sauropod taxa (e.g. Australodocus [Remes, 2007]) and Euhelopus [Wilson \& Upchurch, 2009]), these tend to be bifid structures; as such, MB.R.2091.31 shares this potentially unusual combination of a low, single neural spine with the holotypic anterior dorsal vertebrae of Tendaguria (as well as Moabosaurus).

\section{INTERNAL TISSUE STRUCTURE OF MB.R.2091.31}

The vertebra is pneumatised by several large and small subcircular camerae (Wedel, 2003) that are regularly distributed throughout the bone (Fig. 23A-P). Pneumatic cavities are closely packed and internally separated only by thin walls of bone, with the external bone walls slightly thicker. Distinctly smaller camerae occur only in a few places, in particular in the condyle and lateral to the neural canal, whereas the predominant pneumatic camerae of the vertebral body are considerably larger.

The centrum bears no distinct midline strut of bone, but only a thin median wall that is replaced posteriorly by a dorsoventrally elongate camera with very thin bone walls (Fig. 23K). The condyle is hollowed out by large, rounded camerae that are separated by thin bone walls, and are surrounded by smaller camerae (Fig. 23B, C). Ventrally, these smaller camerae hollow out the parapophyses (Fig. 23D), and posteriorly, they increase in size. The pneumatic camerae are connected to the external surface and extend into the lateral pneumatic fossa of the centrum and into the CDF. At the diapophyseal level, the vertebral 
body becomes transversely narrow, consisting only of a few pneumatic camerae that are separated by very thin bone walls (Fig. $23 \mathrm{E}-\mathrm{H}$ ). The walls of the neural canal apparently have collapsed in places, so that separation to the surrounding camerae is incomplete. Generally, pneumatic camerae of smaller and larger size throughout the vertebra surround and are connected to the neural canal (Fig. 23M).

The prezygapophyses are massive around their articular surface, and become hollowed out at the same level at which pneumatic camera start to develop within the condyle (Fig. 4b). The pneumatic camera of each prezygapophysis opens laterally into the PRCDF and posteriorly excavates the SPRL (Fig. 23C). The postzygapophysis is pneumatised by large camerae at its base, but its posterior half (including the articular surface) is apneumatic (Fig. $23 \mathrm{~K}, \mathrm{~L}$ ). The lateral neural spine is hollowed out by a right and a left pneumatic camera, which close just anterior to the postspinal fossa (Fig. 23G).

Only rounded camerae are present, which are regularly distributed throughout most of the vertebra, and are of varying dimensions. This polycamerate pattern of pneumatisation strongly resembles that observed in the diplodocid Apatosaurus (Wedel, 2003), but differs from other diplodocids (Wedel, 2003; Schwarz, Frey \& Meyer, 2007). The absence of camellae distinguishes MB.R.2091.31 from titanosauriforms such as Giraffatitan (Schwarz \& Fritsch, 2006), Sauroposeidon (Wedel et al., 2000; Wedel, 2003), and derived titanosaurs (e.g. Powell, 1992; Wedel, 2003; Woodward \& Lehman, 2009; Cerda, Salgado \& Powell, 2012).

SAUROPODA MARSH, 1878

EUSAUROPODA UPCHURCH, 1995

MAMENCHISAURIDAE YOUNG \& ZHAO, 1972

\section{WAMWERACAUDIA KERANJEI gen. et sp. nov}

Figs $24-31$

Gigantosaurus robustus Janensch, 1929a

Etymology: The genus name honours the Wamwera, the most populous tribe in the Lindi District, which includes the area of Tendaguru Hill, and caudia is derived from the Greek for tail, in reference to the elements comprising the holotype. The species name is in recognition of the efforts of Mohammadi Keranje, who supervised the excavation of the holotypic individual (Maier, 2003).

Holotype: MB.R.2091.1-30, 3817.1 and 3817.2 (G1-30) - a series of 30 articulated caudal vertebrae, two anterior caudal neural spines, and two incomplete chevrons.

Type locality and horizon: Quarry G, approximately $600 \mathrm{~m}$ south of Tendaguru Hill, Lindi District, southeastern Tanzania (Fig. 1); Upper Dinosaur Member (Upper Saurian Bed), Tendaguru Formation; Tithonian, Late Jurassic (Janensch, 1929a; Bonaparte et al., 2000; Aberhan et al., 2002; Remes, 2007; Bussert et al., 2009).

Referred material: MB.R.2094 (Oa12) - an isolated anterior caudal vertebra; Obolello, Quarry Oa, approximately $15 \mathrm{~km}$ southwest of Tendaguru Hill, Lindi District, southeastern Tanzania (Fig. 1); Upper Dinosaur Member (Upper Saurian Bed), Tendaguru Formation; 
Tithonian, Late Jurassic (Janensch, 1925b, 1929a; Bonaparte et al., 2000; Aberhan et al., 2002; Bussert et al., 2009). Janensch (1929a) mentioned the existence of more than one anterior caudal vertebra from this locality, but only MB.R.2094 remains in the MfN collections (Bonaparte et al., 2000).

Diagnosis: Wamweracaudia can be diagnosed by four autapomorphies (marked with an asterisk), as well as two local autapomorphies: (1) anteriormost caudal ribs curve strongly anterolaterally; (2) dorsal surface of centrum posteriorly excavated in anterior-middle caudal vertebrae*; (3) paired tubercles present on lateral surface of prezygapophysis and on dorsal surface of caudal rib (situated an approximately equal distance between the prezygapophysis and the lateral tip of the caudal rib) in anterior caudal vertebrae; (4) rugosity along the dorsal third of the lateral surface of anterior caudal neural spines, separated from the postspinal rugosity by a vertical groove*; $(5)$ ventral surface of middleposterior caudal centra strongly constricted transversely to form a ridge rather than a distinct, transversely wide surface*; (6) anteroposteriorly elongate ridge on lateral surface of middle-posterior caudal neural spines, just above the level of the postzygapophyses*.

Additional comments: These caudal vertebrae were originally referred to Janenschia robusta by Janensch (1929a), although no basis was given for this attribution. We can only assume that this referral was based on comparisons with the now lost caudal vertebrae from Quarry P. However, it is impossible to discern the morphology of these vertebrae based on the surviving field map of Janensch (Fig. 10) and, as illustrated, they appear most likely to represent middle-posterior caudal centra, making the recognition of shared features difficult. As such, there is currently no basis for referring them to Janenschia (see also 'Discussion - Oversplitting of Tendaguru sauropods?').

\section{DESCRIPTION AND COMPARISONS OF HOLOTYPE MATERIAL OF WAMWERACAUDIA KERANJEI}

\section{Caudal vertebrae}

A sequence of 30 caudal vertebrae is accessioned as MB.R.2091.1-30 (Figs 24-30). The caudal vertebrae are numbered inversely, such that MB.R.2091.30 is the most anterior preserved element and MB.R.2091.1 is the most posterior. Here they are described as caudal vertebrae (Cd) XXX to Cd I. Two additional caudal neural spines from the anterior region of this tail are also preserved and accessioned separately (MfN MB.R.3817.1 and 3817.2). One of them might represent the missing caudal neural spine of Cd XXV; however, the other indicates the presence of at least one additional caudal vertebra. The centra of the first eight caudal vertebrae are incomplete, particularly along their left sides; otherwise, the tail sequence is fairly complete and well preserved (see Table 9 for measurements). Rather than fully describing each caudal vertebra, we record each anatomical feature the first time it can be observed, and then document how this changes along the sequence.

$\mathrm{Cd} X X X$ is the most anterior caudal vertebra preserved and likely represents one of the first few caudal vertebrae (Bonaparte et al., 2000). Little can be gleaned from the highly incomplete and deformed centrum, other than its internal tissue structure is solid, as is that of the neural arch. Subsequent caudal vertebrae do not differ in this regard. Thus, Wamweracaudia lacks the camellate internal structure present in the caudal vertebrae of several derived titanosaurs (Wilson, 2002; Powell, 2003; Wedel, 2003; Mannion et al., 2013). The caudal rib projects mainly laterally and ventrally, although it starts to curve anteriorly 
towards its distal end (note that the very distal tip is missing). As in subsequent caudal vertebrae, the ventral margin of the caudal rib is oriented horizontally, or even deflected ventrally, thereby lacking the dorsal deflection seen in dicraeosaurids, rebbachisaurids and some titanosauriforms (Mannion, Upchurch \& Hutt, 2011; Whitlock, 2011a; Mannion \& Barrett, 2013). The caudal rib is supported from below by an ACDL, but it is not possible to determine whether this was a prominent structure, or if a PCDL was also present, because of poor preservation in this region. The anterior surface of the rib is dorsoventrally concave, although this does not form a sharp lipped fossa, and the depth of concavity has clearly been accentuated by crushing. The posterior surface of the rib is dorsoventrally convex and lacks fossae. The dorsal margin of the rib has been sheared ventrally along part of its length, but is formed from a prominent, sharply defined PRDL. Distinct PRDLs and sometimes other diapophyseal laminae are known in the anterior caudal vertebrae of numerous neosauropods (Wilson, 1999, 2002; Chure et al., 2010; Mannion et al., 2011, 2013, 2017; Whitlock, 2011a), but are also present in some non-neosauropod eusauropods, e.g. Lapparentosaurus (MNHN MAA 169: PDM pers. obs. 2011) and Mamenchisaurus (Ouyang \& Ye, 2002: fig. 30). Prezygapophyses project mainly anteriorly and slightly dorsally, and are supported from below by well defined, anterodorsally oriented and medially deflected CPRLs. Each prezygapophysis is an anteroposteriorly short, mediolaterally thin structure, with a flat, medially-facing articular surface and mildly dorsoventrally convex lateral surface. A poorly defined PRCDF is present on the anterior surface of the neural arch. This is situated lateral to the prezygapophysis, posterodorsal to the CPRL, and ventrolateral to the PRDL. Postzygapophyses are mediolaterally thin structures. Their flat articular surfaces face mainly laterally, but also slightly posteroventrally. A presumed prezygapophysis is attached to the right postzygapophysis in approximately natural articulation. As Cd XXIX preserves both prezygapophyses, this indicates the presence of an additional, unpreserved caudal vertebra in between Cd XXX and Cd XXIX. A mediolaterally thick, dorsoventrally short hyposphenal ridge extends from the ventral midline of the postzygapophyses down to the dorsal margin of the posterior neural canal. The possession of a hyposphenal ridge in anterior caudal vertebrae is the plesiomorphic condition in sauropods, but is lost in some somphospondylans and many rebbachisaurids (Upchurch, 1998; Mannion et al., 2011, 2012, 2013). Wamweracaudia differs from most non-macronarians in which the hyposphene forms a slender ridge (Mannion et al., 2013), although Omeisaurus shares the presence of a blocklike hyposphene (He, Li \& Cai, 1988: fig. 34b). A small, shallow, subcircular fossa is present either side of the postzygapophyses on the posterior surface of the neural arch, bounded anteriorly by a short and rounded PODL. The neural spine projects dorsally and slightly posteriorly. The anterior surface of the base of the neural spine is poorly preserved, but there is a remnant of a SPRL. This seems to merge into the anterolateral margin of the neural spine dorsally. Although poorly preserved, the remaining anterior face of the neural spine is composed mainly of a rugose surface that expands mediolaterally towards the dorsal tip of the neural spine. At the base of the neural spine, on the anterior half of the lateral surface, is a small, shallow SDF that opens mainly dorsally. This SDF is bounded anteriorly by the SPRL, posteriorly by the PODL, and ventrolaterally by the PRDL. Anteroposteriorly elongate, mediolaterally thin SPOLs form the posterolateral margins of the neural spine, and define an extremely deep postspinal fossa. At approximately one-third of the way up the neural spine, this fossa begins to be 'infilled' by a postspinal rugosity that extends all of the way to the neural spine apex. The lack of distinct pre- and postspinal ridges differs from most diplodocoids and many titanosaurs (Mannion et al., 2013). The lateral surfaces of the spine lack any ridges or fossae, but at approximately two-thirds of the way up the spine, at the 
point where the SPOLs begin to fade out, the spine increases in mediolateral thickness, with the lateral surfaces developing a rugose texture similar to that of the pre- and postspinal rugosities. The dorsal margin of the spine is strongly convex transversely in anterior view, and gently convex in lateral view. The latter is the condition in most sauropods, whereas the dorsal margins of the anterior caudal neural spines of several Middle Jurassic eusauropods are strongly concave or sinuous in lateral view, e.g. Cetiosauriscus (NHMUK R3078: PDM \& PU pers. obs. 2011) from the UK, Jobaria (MNN specimens: PDM pers. obs. 2012) and Spinophorosaurus (Remes et al., 2009: fig. 3c) from northern Africa, and MACN-CH 1299 (PDM \& PU pers. obs. 2013) from Argentina.

Although also incomplete and crushed, the centrum of Cd XXIX is clearly strongly procoelous, with a deeply concave anterior surface and a strongly developed posterior ball. Prominent procoely in anterior caudal vertebrae is a feature otherwise restricted to derived titanosaurs, the basal somphospondylan Dongbeititan, turiasaurs, Bellusaurus, and the mamenchisaurids Chuanjiesaurus, Klamelisaurus and Mamenchisaurus (McIntosh, 1990; Upchurch, 1995, 1998; Salgado et al., 1997; Wilson, 2002; Sekiya, 2011; Whitlock, D’Emic \& Wilson, 2011; Mannion et al., 2013). Furthermore, the highest Condylar Convexity Index (CCl $=$ anteroposterior length of posterior condylar ball divided by mean radius of the condyle [i.e. mediolateral width + dorsoventral height of articular surface, divided by 4]; Mannion et al., 2013) value of Wamweracaudia (0.62) is closest to the condition in derived titanosaurs, as well as Moabosaurus and Mamenchisaurus (see Table 10). The caudal rib curves strongly anterolaterally, as well as projecting ventrally, and extends beyond the anterior margin of the centrum. Among Sauropoda, the Gondwanan diplodocids Tornieria (Remes, 2006: fig. 5) and Leinkupal (Gallina et al., 2014) also show anteriorly curved anterior caudal ribs, but only in the Late Cretaceous Chinese titanosaur Xianshanosaurus is this as strongly developed as in Wamweracaudia (Lü et al., 2009; Mannion et al., 2013); as such, this feature is regarded as an autapomorphy of Wamweracaudia (see also 'Discussion: Turiasaurs outside of Euamerica?'). Although the caudal rib is deflected ventrally, it contrasts with the condition in diplodocids whereby the distal tip of the rib projects ventrally, giving the ventral margin a concave profile in anterior/posterior view (Gallina et al., 2014). As in Cd XXX, the caudal rib has a dorsoventrally concave anterior surface and dorsoventrally convex posterior surface. There is evidence for a probable ACDL, and the PRDL remains prominent. On the dorsal surface of the caudal rib, level with the lateral margin of the centrum, is a small circular fossa. This fossa appears to have been formed from abnormal suturing of the neural arch and caudal rib, and is not present in the other caudal vertebrae. A tubercle is also present on the lateral surface of the lower part of the prezygapophysis/uppermost part of the PRDL (the 'upper process for muscular attachment' of Bonaparte et al. [2000]) (see the description of $\mathrm{Cd}$ XXVI for further discussion of this feature). The neural canal has undergone some crushing, but appears to form a dorsoventrally compressed ellipse. Prezygapophyses do not differ significantly from those in $\mathrm{Cd} X \mathrm{XX}$, although they are slightly more robust structures, with a D-shaped cross section. The articular surfaces of the postzygapophyses are mildly concave dorsoventrally, and a block-like hyposphenal ridge is present. The fossae described on the anterior and posterior surfaces of the neural arch in Cd XXX are all present in Cd XXIX, but there is no longer a SDF on the lateral surface at the base of the neural spine. A prespinal fossa is present, and is infilled by a prespinal rugosity. SPRLs form the anterolateral margins of the neural spine, gradually fading out dorsally. As in Cd XXX, there is a SPOL-bounded, deep postspinal fossa infilled with a postspinal rugosity.

The caudal rib of Cd XXVIII is slightly mediolaterally shorter than in preceding caudal vertebrae, but still projects strongly anterolaterally, reaching or slightly surpassing the 
anterior margin of the procoelous centrum. The gently concave distal articular surface of the rib forms an anteroposteriorly elongate D-shape, with a flat dorsal margin in lateral view. This cross section is maintained throughout the length of the rib. No centrodiapophyseal laminae are present, although there is a reduced PRDL. The fossae on the anterior and posterior surfaces of the neural arches are extremely shallow. Prezygapophyses project anteriorly and a little dorsally, extending well beyond the anterior margin of the centrum. The tubercle on the lateral surface of the prezygapophysis is still present. The neural spine does not differ greatly from that of preceding vertebrae, although the ventral portions of the pre- and postspinal fossa are not infilled with rugosities. In addition, the posterior deflection of the neural spine is more marked, such that the anterodorsal margin of the neural spine is posterior to the posterior margin of the postzygapophyses. This morphology is shared with the anterior caudal vertebrae of several non-neosauropod eusauropods (e.g. Chuanjiesaurus, Mamenchisaurus and Shunosaurus), diplodocids, and some derived titanosaurs (Salgado et al., 1997; Mannion et al., 2013).

Cd XXVII includes an incomplete, but largely undistorted centrum. As in preceding vertebrae, the centrum is deeply concave anteriorly and has a strongly convex posterior surface. The lateral surface of the centrum is dorsoventrally convex and gently concave anteroposteriorly; it lacks ridges or fossae. The caudal rib curves strongly anterolaterally, although it does not extend as far as the anterior margin of the centrum. There is no longer any ventral deflection of the caudal rib, and it has an elliptical cross section, with the long axis of this ellipse oriented anteroposteriorly. Only a very weakly developed PRDL remains, and there is no evidence for centrodiapophyseal laminae. CPRLs are no longer present as distinct laminae; instead, the anterior surface of the neural arch, below the prezygapophyses, is composed of flat, almost featureless bone. The tubercle on the lateral surface of the prezygapophysis is slightly more posteriorly placed than in preceding caudal vertebrae, and forms an anteroposteriorly elongate rugosity. SPRLs are mediolaterally thin, anteroposteriorly prominent structures forming the anterolateral margins of the neural spine. In between them is a deep prespinal fossa, with the dorsal two-thirds infilled with a rugosity. The SPRLs disappear at the level of the mediolateral expansion of the neural spine, near its apex. The postspinal fossa is deep at the base of the neural spine, but is rapidly infilled with a rugosity. SPOLs are bifid, with the medial branches forming the posterolateral margins of the neural spine, fading out at approximately midheight. The lateral branches extend dorsally and slightly anteriorly onto the lateral surfaces of the neural spine, although they extend dorsally only a short distance. A bifid SPOL is a potentially unusual feature in caudal vertebrae, but its unambiguous presence (see below) in only one caudal vertebra precludes its inclusion in our diagnosis. A vertical groove separates the postspinal rugosity and the lateral rugosities along the mediolaterally expanded, dorsal portion of the neural spine: this combination of lateral rugosity and groove is regarded as an autapomorphy of Wamweracaudia.

The caudal rib of Cd XXVI projects mainly laterally, although there is a small degree of anterior curvature. The neural canal is subcircular at its anterior end, but forms a dorsoventrally elongate ellipse at its posterior opening. A PRDL is now entirely absent. As well as the rugosity on the lateral surface of the prezygapophysis, there is also a second tubercle on the dorsal surface of the caudal rib, situated approximately equidistant between the prezygapophysis and the lateral tip of the caudal rib (the lower process for muscular attachment' of Bonaparte et al. [2000]). A similar pair of upper and lower tubercles develops along the sequence in the somphospondylans Baurutitan (Kellner et al., 2005: fig. 13) and Huabeisaurus (D'Emic et al., 2013), and the lower tubercle is present in the anterior caudal 
vertebrae of several other titanosaurs (e.g. Epacthosaurus [Martínez et al., 2004: figs 6-7] and Mendozasaurus [D'Emic et al., 2013]), the diplodocid Tornieria (Remes, 2006: fig. 5a), the turiasaur Losillasaurus (MCNV Lo: PDM \& PU pers. obs. 2009), and also in Mamenchisaurus (Ouyang \& Ye, 2002: fig. 30). An upper tubercle also appears to be present in Omeisaurus (He et al., 1988: fig. 34). We regard the presence of these paired tubercles as a local autapomorphy of Wamweracaudia. There is some evidence for a bifid SPOL, but this region is too poorly preserved to be certain. In Cd XXV, the caudal rib projects only laterally, and the prezygapophyses project anterodorsally and extend well beyond the anterior margin of the centrum. In other regards, these vertebrae do not differ significantly from Cd XXVII. The two additional caudal neural spines (MB.R.3817.1 and MB.R.3817.2) are from approximately this position in the tail, based on their size and morphology. Both display the posterolateral groove separating the posterior and lateral rugosities on the dorsal portion of the neural spine.

The neural canal of Cd XXIV is elliptical at both ends, but is dorsoventrally compressed anteriorly and dorsoventrally elongate posteriorly. The dorsal surface of the centrum (i.e. floor of the neural canal), towards the posterior end of the neural canal, is excavated, forming a moderately deep, elliptical fossa. This fossa is present in subsequent caudal vertebrae too, and it might represent an opening for the basivertebral veins (Manning, Egerton \& Romano, 2015) or possibly the arterial channels (Wintrich, Scaal \& Sander, 2017). A similar fossa has also been described in the anterior caudal vertebrae of two Middle Jurassic eusauropods: an isolated vertebra from the Aalenian of the UK (Manning et al., 2015), and material from the Malagasy Bathonian taxon 'Bothriospondylus madagascariensis' (Mannion, 2010). However, as neither of these occurrences resemble the anterior caudal vertebrae of Wamweracaudia (for example, both forms have amphicoelous centra), we include this feature in our diagnosis of the Tendaguru taxon. The rugosities described on the lateral surfaces of prezygapophyses in preceding vertebrae are still present, but now form anteroposteriorly elongate ridges and are situated more posteriorly along the arch, less directly associated with the prezygapophyses. The lower tubercle, on the caudal rib, is still present, though less prominently developed. Again, there is some evidence for a bifid SPOL, but poor preservation precludes confirmation of this feature.

A hyposphenal ridge is completely absent from Cd XXIII onwards. There is a very weakly developed lateral branch of the SPOL in Cd XXIII, suggesting that the SPOL is probably genuinely bifid on Cds XXVI-XXIV. Cd XXII is the first vertebra to preserve a complete centrum. The anterior surface of the centrum is concave, whereas the posterior surface is flat to irregularly convex. Thus, Wamweracaudia does not retain procoely throughout all of its anterior caudal vertebrae (defined as those retaining a caudal rib), in contrast to Mamenchisaurus and derived titanosaurs (Mannion et al., 2013). A shallow fossa is present on the lateral surface of the centrum, ventral to the caudal rib. A lateral fossa in anterior caudal vertebrae otherwise appears to be restricted to diplodocoids, brachiosaurids, and some derived titanosaurs (McIntosh, 1990; Upchurch, 1995; Upchurch \& Mannion, 2009; D’Emic, 2012; Ibiricu et al., 2012; Mannion \& Barrett, 2013; Mannion et al., 2013; Poropat et al., 2016). The ventral surface of the centrum is slightly distorted, but preserves remnants of posterior chevron facets. The posterior half of the ventral surface appears to be mildly concave mediolaterally, whereas anteriorly this surface is flat to convex. The neural canal is subcircular at both openings, and the neural spine is a little more posteriorly directed than in preceding caudal vertebrae.

Numerous small, shallow vascular foramina pierce the lateral and ventral surfaces of the centrum of $\mathrm{CdXXI}$, and the fossa ventral to the caudal rib is again present. On the dorsal 
surface of the centrum, a deep, anteroposteriorly elongate fossa is again present along the posterior half of the neural canal. The anterior surface of the centrum of $\mathrm{Cd} X X$ is concave, whereas the posterior surface is mildly concave with some irregular bulges. The ventral surface is mildly concave mediolaterally along the midline of the posterior two-thirds of the centrum, but there are no ventrolateral ridges, contrasting with the condition in several diplodocoids and many derived somphospondylans (McIntosh, 1990; Upchurch, 1995, 1998; Upchurch et al., 2004; Mannion \& Barrett, 2013; Mannion et al., 2013). Only a very gentle fossa is present on the lateral surface of the centrum of $\mathrm{CdXX}$, and the caudal rib is greatly reduced. $\mathrm{Cd}$ XIX does not differ greatly from $\mathrm{Cd} \mathrm{XX}$, although there is no longer a lateral fossa on the centrum, and a horizontal ridge is formed along the arch-centrum junction.

The anterior and posterior articular surfaces of the centrum of Cd XVIII are gently concave. Anterior and posterior chevron facets are present, and the ventral surface is very mildly concave mediolaterally. A ridge-like, greatly reduced caudal rib is present, and the horizontal ridge at the arch-centrum junction is more sharply defined than on $\mathrm{Cd}$ XIX. The ridge on the lateral surface of the neural arch is also still present, although it is less prominent than in preceding vertebrae. The neural spine projects posterodorsally, with the postzygapophyses situated beyond the posterior margin of the centrum. There is no longer a well-defined postspinal fossa.

The ventral surface of the centrum of $\mathrm{Cd} X \mathrm{VII}$ is flat, lacking the concavity seen in preceding vertebrae. The caudal rib is almost entirely absent, and a weak horizontal ridge is present at approximately two-thirds of the way up the centrum, extending for most of the centrum length. The latter is a feature of the middle caudal centra of a number of eusauropods, including Cetiosauriscus and Omeisaurus (Upchurch \& Martin, 2003), diplodocids, the rebbachisaurid Demandasaurus (Pereda Suberbiola et al., 2003), basal macronarians (Mannion et al., 2013), and the titanosaur Andesaurus (Mannion \& Calvo, 2011). By Cd XVI, the caudal rib is fully absent and the neural spine starts to become more elongate anteroposteriorly, with a less pronounced mediolateral expansion near the dorsal end. The neural arch of this element, and all middle caudal vertebrae, is situated at an approximately equal distance from the anterior and posterior margins of the centrum, differing from the anteriorly biased middle caudal neural arches of Galveosaurus + Titanosauriformes (Calvo \& Salgado, 1995; Upchurch, 1995, 1998; Salgado et al., 1997; Mannion et al., 2013).

The ridge present on the neural arch in preceding vertebrae, level with the prezygapophyses, is absent from Cd XV onwards. In dorsal view, the neural spine gently increases in mediolateral width towards its posterior margin, and in lateral view is approximately rectangular. The neural spine is anteroposteriorly shortest at its base, expanding dorsally so that it approximately doubles in length. Mannion et al. (2013) noted that this morphology is otherwise known only in the Chinese mamenchisaurids Chuanjiesaurus and Mamenchisaurus, as well as the subsequently described Qijianglong (Xing et al., 2015: fig. 14e), although it is also present in at least one middle caudal vertebra of the Chinese non-mamenchisaurid eusauropod Bellusaurus (Mo, 2013: pl. 42), and in the distal-most middle caudal vertebrae of Cetiosauriscus (NHMUK R3078: PDM pers. obs. 2017).

In Cd XIV, the ventral surface of the centrum becomes gently convex mediolaterally. Prezygapophyses do not extend beyond the anterior margin of the centrum, and prespinal and postspinal rugosities are still present. By Cd XIII, the ridge at the arch-centrum junction is reduced to a low bulge, and the dorsalmost point of the anterior surface of the neural spine forms a bulbous projection. In Cd XII, there is a bulbous projection at the dorsalmost 
portion of the posterior surface of the neural spine. The prespinal fossa is bifurcated by a mediolaterally thin, vertical ridge.

In $\mathrm{Cd} \mathrm{XI}$, the ventral surface begins to become strongly constricted mediolaterally. The ridge on the lateral surface of the centrum, two-thirds up from the ventral margin, is more prominently developed than in preceding vertebrae. There is a gentle ridge on the lateral surface of the neural spine, just above the level of the postzygapophyses, oriented posterodorsally at an angle of approximately $30^{\circ}$ to the horizontal. Incipient versions of this ridge appear to be present in $\mathrm{Cd}$ XIII and $\mathrm{Cd}$ XII, and its presence is considered an autapomorphy of Wamweracaudia. The posterodorsal bulge on the neural spine is again present, but an anterodorsal one is absent.

The anterior and posterior articular surfaces of the centrum of $\mathrm{Cd} X$ are gently concave, particularly centrally, with the anterior concavity slightly deeper than the posterior one. Chevron facets are present, but weakly developed, and there is no ridge along the archcentrum junction. Postzygapophyses form facets on the posteroventral margin of the spine; their anterior margins are level with the posterior margin of the centrum. A ridge situated above the postzygapophysis, as described in $\mathrm{Cd}$ XI, is still present, as are a postspinal rugosity and posterodorsal bulge on the neural spine. The neural spine expands anteroposteriorly towards its dorsal end and extends to approximately midlength of the subsequent caudal centrum. This posterior extension to the midlength of the subsequent vertebra is also a feature of several non-neosauropods (Remes et al., 2009; Mannion et al., 2013), including the Chinese taxa Chuanjiesaurus, Mamenchisaurus, Omeisaurus, Shunosaurus and Qijianglong (Sekiya, 2011: figs 32-33; Mannion et al., 2013; Xing et al., 2015: fig. 14f), the African genera Jobaria and Spinophorosaurus (Remes et al., 2009), and the Indian taxon Barapasaurus (Bandyopadhyay et al., 2010: fig. 7).

The prezygapophyses on Cd IX extend a short distance beyond the anterior margin of the centrum, and the ridge above the postzygapophyses extends further anteriorly than in preceding vertebrae, where it becomes mainly horizontally oriented. The prespinal fossa appears to be absent in Cd VIII and there are no longer distinct SPRLs. Similarly, there is no longer a postspinal fossa or SPOLs, although the posterior surface of the neural spine remains rugose. In Cd VII, the lateral ridge on the centrum is poorly defined and the ventral surface of the centrum is more strongly constricted mediolaterally than in preceding vertebrae, such that it forms a near ridge-like structure along the middle third. This ventral morphology is unknown in other sauropods and is thus considered an autapomorphy of Wamweracaudia.

The anterior articular surface of the centrum of $\mathrm{Cd} \mathrm{VI}$ is mildly concave, with a more deeply developed central concavity. In contrast, the posterior articular surface is irregularly flat to mildly convex in parts, becoming concave centrally. Weakly developed posterior chevron facets are still present. Prezygapophyses do not extend beyond the anterior margin of the centrum, contrasting with the anteriorly extensive processes of somphospondylans (Mannion et al., 2013). The autapomorphic ridge on the lateral surface of the neural spine, above the level of the postzygapophyses, now extends for most of the anteroposterior length of the neural spine. Cds V-III provide no additional anatomical information. There is no well-developed ridge on the lateral surface of the centrum of $\mathrm{Cd} I \mathrm{I}$, and there is a mediolaterally narrow ridge along the ventral half of the anterior surface of the neural spine. $\mathrm{Cd}$ I does not differ in any substantial way to preceding caudal vertebrae.

Chevrons 
Two distally incomplete chevrons, along with a fragment of distal blade that possibly belongs to one of them, are preserved (Fig. 30; see Table 11 for measurements). They are labelled as G28 and G27, and their size approximately matches those positions in the caudal sequence. The proximal ends of both are bridged by bone, differing from the open condition that characterises the majority of neosauropods (Upchurch, 1998; Wilson, 2002; Mannion \& Calvo, 2011). The proximal articular surface of each chevron is strongly convex anteroposteriorly, forming a roller surface rather than distinct anterior and posterior facets. In G28, the haemal canal is a dorsoventrally elongate oval shape, transversely thinning distally. In contrast, the haemal canal in G27 is much shorter dorsoventrally and closer to a circular shape. Although both chevrons are incomplete, the haemal canal depth to chevron length ratio is clearly less than 0.4 , contrasting with the deep haemal canals seen in proximal chevrons of most titanosauriforms (Wilson, 2002; Mannion et al., 2013). Neither haemal canal is situated within a fossa, differing from the condition present in the turiasaur Zby (Mateus et al., 2014). There are no ridges or bulges on the lateral surfaces of the proximal rami, in contrast to those seen in some derived titanosaurs (Santucci \& Arruda-Campos, 2011; Poropat et al., 2016). The distal blade possesses the mediolaterally compressed morphology that is typical of most sauropods. A proximodistally oriented midline ridge is present along the anterior and posterior surfaces of the blade, but there are no ridges along the lateral surfaces.

\section{DESCRIPTION OF MATERIAL REFERABLE TO WAMWERACAUDIA KERANJEI}

MB.R.2094 is a nearly complete anterior caudal vertebra (Fig. 31) from Quarry Oa (see also Janensch, 1929: fig. 16), missing its left caudal rib, right prezygapophysis and left postzygapophysis. Although mostly well preserved, some regions have been eroded. It is from a much larger animal than the type individual of Wamweracaudia (see Table 9 for measurements).

The internal tissue structure is fine and spongy, lacking camellae. The centrum is procoelous, with a deeply concave anterior surface and strongly convex posterior ball $(\mathrm{CCl}=$ 0.5). Ventrally, the centrum is transversely flat and gently concave anteroposteriorly, whereas the lateral surface is dorsoventrally convex and anteroposteriorly concave. Both surfaces lack ridges or excavations. There is some evidence for anterior chevron facets, and so it is likely that the absent posterior chevron facets have been eroded away. A simple caudal rib projects laterally and curves anteriorly, as in the anterior caudal vertebrae of Wamweracaudia, although it does not extend as far as the anterior margin of the centrum.

At its anterior end, the neural canal appears to be semicircular, with a flat ventral margin, whereas the posterior opening is subcircular. There is a small fossa on the neural canal floor, close to the posterior end. Similar excavations are also present in the holotype of Wamweracaudia. There is no evidence for a distinct PRDL, which probably indicates that this vertebra is not one of the anteriormost caudal vertebrae in the tail (see also Bonaparte et al., 2000), but might alternatively reflect the abraded nature of the element. A prominent tubercle is situated on the dorsal surface of the caudal rib, at approximately midlength. Prezygapophyses project strongly anterodorsally, extending only a short distance beyond the anterior margin of the centrum. Their flat articular surfaces face dorsomedially. A bulge-like rugosity is situated on the lateral surface of the prezygapophysis. As such, this vertebra also shows the upper and lower tubercles present in the holotype of Wamweracaudia. Based on the position of their development along the holotypic sequence, MB.R.2094 is probably approximately $\mathrm{Cd} 5$. The postzygapophyseal articular surfaces are mediolaterally concave and 
face mainly ventrally and slightly laterally. A shallow subcircular fossa is present on the posterolateral surface of the neural arch, lateral to the postzygapophysis. A block-like hyposphenal ridge appears to be present, but is partly concealed by plaster.

The neural spine projects mainly dorsally. SPRLs are anteroposteriorly expanded at their bases but are reduced dorsally, gradually merging into the anterolateral margins of the neural spine. Single SPOLs form the posterolateral margins of the spine. Pre- and postspinal fossae are present and both are infilled with rugosities, except at their ventral ends. Towards its apex, the neural spine expands mediolaterally. The lateral surface of the neural spine lacks ridges or fossae. At the point at which the SPOLs start to merge into the posterolateral margin of the neural spine, a vertical groove is present between the postspinal rugosity and posterolateral margin, as was also observed in Wamweracaudia. The dorsal surface of the neural spine is convex in all directions.

\section{INDETERMINATE TENDAGURU MATERIAL PREVIOUSLY REFERRED TO JANENSCHIA}

Sauropod remains are known from dozens of localities within the Tendaguru Formation (Janensch, 1929a; Heinrich, 1999b; Maier, 2003), although sites producing elements that have been attributed to Janenschia are much less common (Bonaparte et al., 2000). As well as the type locality (Quarry B), and quarries Oa (referred material of Wamweracaudia) and P, material has been referred to Janenschia from quarries dd, G, IX, NB, Nr. 5 and Nr. 22 (Janensch, 1922, 1925, 1929a, 1961; Bonaparte et al., 2000), but cannot be unequivocally referred to that taxon, Tendaguria, or Wamweracaudia. Below, we describe and discuss the taxonomic status of these materials.

\section{Quarry Nr. 5}

A near complete, articulated right manus (MB.R.2093; Figs 32-35) was collected from site Nr. 5, which was described as near to the Janenschia type locality (Janensch, 1922, 1961; Bonaparte et al., 2000). Its referral to Janenschia was based on its proximity to the type and comparisons to material from Quarry $P$ (Janensch, 1922; Bonaparte et al., 2000). Unfortunately, as outlined above (see also Bonaparte et al., 2000), almost all of the manual material from Quarry $P$ has been destroyed, and these elements were never described or fully figured. The remaining manual material lacks autapomorphies. Thus, it is not possible to confidently refer the manus to Janenschia, and it clearly differs from the only other Tendaguru taxon to preserve assignable manual material, i.e. Giraffatitan (Janensch, 1922, 1961).

In articulation, the metacarpals display a 'horseshoe'-shaped structure in proximal view (Fig. 34), forming the vertically-oriented 'colonnade' arrangement that characterises most eusauropods (Upchurch, 1995, 1998, Wilson \& Sereno, 1998; Wilson, 2002, 2005a). Metacarpal II is the longest metacarpal, followed by metacarpal III and then IV, although metacarpal III is damaged and its length might be slightly underestimated. Metacarpal V is the shortest element, but is only very slightly shorter than metacarpal I. The ratios of the lengths of metacarpals I to III and IV are less than 1.0, comparable to most eusauropods, with the exception of some titanosaurs (Upchurch, 1998; Wilson \& Sereno, 1998; Poropat et al., 2015b). The phalangeal formula is $2-2-1-1-1$, as is the case in most neosauropods (sometimes further reduced to 2-1-1-1-1), with the exception of titanosaurs, which have lost their manual phalanges (Upchurch, 1998; Tschopp et al., 2015b). Non-neosauropod eusauropods typically retain two phalanges on at least digits I-IV (Upchurch, 1998; Wilson \& Sereno, 1998; Wilson, 2002), including Turiasaurus (Royo-Torres et al., 2006). The non- 
ungual phalanges of MB.R.2093 are mediolaterally wider than proximodistally long, as in other eusauropods (Wilson \& Sereno, 1998). Below, the manus is described as if the constituent elements were held in a horizontal position, rather than their in vivo vertical position. Metacarpals are described with the long axis of the distal end oriented mediolaterally. Measurements are provided in Tables 12 and 13.

The proximal end of metacarpal I is ' $D$ '-shaped, with a flat lateral margin that is dorsoventrally taller than the convex medial margin. It lacks the strongly dorsoventrally compressed morphology seen in several titanosauriforms (Apesteguía, 2005a; Mannion \& Calvo, 2011). Its gently rugose proximal articular surface is flat to very mildly convex. The dorsal and medial surfaces curve strongly into one another for most of the length of the metacarpal, only becoming truly distinct towards the distal end. Along the proximal half, the subtriangular shaped lateral surface is concave and striated. The dorsal and ventral margins of this subtriangular lateral region form low ridges. The dorsal ridge fades out at midlength, but the ventral ridge expands to form a prominent projection at approximately two-thirds of the metacarpal length. Ventrally, the metacarpal is flat to mildly concave along its proximal half and flat distally. The metacarpal is also twisted along its axis, such that the proximal half of the ventral surface faces ventrally whereas the distal half faces ventrolaterally. The lateral distal condyle is dorsoventrally shorter than the medial one, but extends further distally, giving the metacarpal a strongly bevelled distal end in dorsal view. This contrasts with the condition in titanosauriforms, whereby the distal end is perpendicular to the long axis of the shaft (Wilson, 2002). The dorsolateral margin of the distal end lacks the rounded bulges that characterise metacarpal I of Turiasaurus (Mateus et al., 2014). There is a fairly prominent ventral notch separating the two distal condyles, although no concavity extends proximally along the ventral surface, differing from the condition in the titanosaur Argyrosaurus (Mannion \& Otero, 2012). The distal articular surface is strongly convex dorsoventrally and concave mediolaterally. It extends onto the dorsal surface, with this extension most prominent along the medial half. This dorsal extension contrasts with the condition observed in most titanosauriforms, in which the articular surface is restricted to the distal end (Salgado et al., 1997; D’Emic, 2012).

Metacarpal II has a triangular shape in proximal view, with the apex of this approximately isosceles triangle pointing ventrally. This triangular cross section is maintained until midlength, at which point the metacarpal becomes elliptical. The lateral and medial surfaces of the proximal half are striated and rugose. Ridges are present along the dorsomedial and dorsolateral margins for much of the metacarpal length, and there is also a ridge along the ventrolateral margin of the proximal half. A rugosity is present on the ventromedial margin, a short distance from the distal end. The lateral distal condyle projects slightly further distally than the medial condyle, but the two condyles are more equidimensional than in metacarpal I. A ventral notch is present at the distal end. The distal articular surface is gently concave mediolaterally and dorsoventrally convex, with a medially biased extension onto the dorsal surface of the metacarpal.

Metacarpal III has been glued back together after breakage around midlength; however, some material is now clearly missing from the shaft and thus its length is probably very slightly underestimated. The proximal end of metacarpal III is triangular, with the apex pointing ventromedially. As such, it lacks the mediolaterally wide trapezoidal shape that characterizes brachiosaurids (Mannion et al., 2017). The triangular cross section is maintained along the proximal half, with a bulbous ventral margin. A ridge is present along the dorsomedial and dorsolateral margins of the proximal two thirds of the metacarpal. The distal condyles project approximately the same distance distally. The distal articular surface 
is dorsoventrally convex and mildly concave mediolaterally, with a prominent, ventral notch. There is a medially biased dorsal extension of this surface. In distal end view, the metacarpal decreases in dorsoventral height along its lateral half, and has a flat medial margin and convex lateral margin.

Metacarpal IV has a subtriangular or wedge-shaped proximal outline, with the lateral and medial margins comprising the longest dimensions of this shape. As such, it lacks the 'chevron' shape seen in brachiosaurids (D'Emic, 2012) and the titanosaur Saltasaurus (PVL 4017-123, -133 \& -183: PDM \& PU pers. obs. 2013). The ventral margin is the shortest diameter of this wedge shape and the dorsal margin is convex. This cross section is maintained along the proximal half of the metacarpal. The ventral tip of this triangle extends as a thickened, ridge-like surface along the ventromedial margin of the metacarpal, fading out just beyond midlength. Along the proximal third, there is a striated triangular, mildly concave lateral surface, bounded by a dorsal ridge, and an irregular medial surface. Just before midlength, at the point where the metacarpal twists along its axis, ridges form along the dorsomedial and dorsolateral margins, extending a short distance distally. As in previously described metacarpals, there is a ventral notch and medially biased dorsal expansion of the distal surface. The distal end has an unusual morphology, similar to that observed in Mc. III. The medial margin is flat and the metacarpal decreases in dorsoventral height towards its lateral margin, such that it has a subtriangular shape in distal end view. Although we do not erect a new taxon, we regard this distal end morphology in metacarpals III and IV as a probable autapomorphy of MB.R.2093.

Metacarpal $V$ has a D-shaped proximal end, with a flat, ventromedially facing margin forming the long axis of this shape. This D-shape is maintained along the proximal half, before the metacarpal becomes more elliptical. The medial surface of the proximal half is heavily striated and is bounded by a dorsomedial ridge that continues in a reduced form along most of the metacarpal length. A broad, prominent ventromedial ridge also bounds this striated region. This ridge is reduced at approximately midlength, where the metacarpal twists along its axis, and fades out a short distance before the distal end, at which point it is situated approximately on the midline of the ventral surface. There is a small bulge on the ventral surface, near to the very distal end, close to the medial margin. The distal end is not divided into two condyles. The distal articular surface is fairly flat mediolaterally and dorsoventrally convex, extending onto the dorsal surface with a slight medial bias.

Manual phalanx I-1 is proximodistally short, with the medial margin shorter than the lateral one. Its proximal articular surface is irregular, but overall it is mediolaterally convex and dorsoventrally flat. In proximal end view, the medial, dorsal and lateral margins all meet at approximately right angles, whereas the ventrolateral corner is convex. As such, the lateral margin is much shorter dorsoventrally than the medial one. There is no lapet-like projection from the proximodorsal margin, overhanging the proximal articular surface, in contrast to other basal eusauropods, including turiasaurs (e.g. Mateus et al., 2014). The dorsal surface of the phalanx is irregularly flat, whereas the ventral surface is proximodistally concave. A wide ventral concavity divides the distal end into two condyles. The distal articular surface is fairly flat dorsoventrally and mediolaterally, although it becomes convex close to the lateral margin.

The proximal end of phalanx II-1 is approximately D-shaped, with a flat medial margin; it decreases in dorsoventral height laterally. The proximal articular surface is irregularly convex. In dorsal view, the lateral margin of the phalanx is concave as a result of the lateral expansion of the distal end. The ventral surface is proximodistally concave. In distal end view, the phalanx is subrectangular, with the long axis oriented mediolaterally. The distal 
articular surface is mildly concave mediolaterally, becoming gently convex towards its margins. It is not divided into distinct condyles.

Phalanx III-1 has a semicircular proximal end, with a relatively flat ventral margin. The proximal articular surface is mildly concave and the ventral surface is concave in all directions. In dorsal view, the medial and lateral margins of the phalanx are concave as a result of mediolateral expansion of the distal end. The distal articular surface is strongly convex mediolaterally, although centrally this surface is flat to mildly concave.

Whereas the proximal end surface of phalanx IV-1 is flat to mildly convex, all remaining surfaces are convex, and there is no distinct distal articular surface. The phalanx decreases in mediolateral width distally, such that it has a semicircular shape in dorsal view.

Phalanx V-1 is considerably larger than IV-1; thus, it is possible that the two phalanges are incorrectly positioned. The proximal end of phalanx $\mathrm{V}-1$ has an elliptical outline, with an irregularly flat articular surface. In dorsal view, the phalanx decreases in mediolateral width distally. The ventral surface is proximodistally concave and the distal end is strongly convex.

Phalanx I-2 is a large ungual claw that in life articulation would have been deflected laterally. It is 0.66 of the length of metacarpal I, differing from the reduced ungual claws seen in titanosauriforms (Upchurch et al., 2004). It decreases in dorsoventral height distally, although this shortening is mainly restricted to the dorsal margin. The ventral margin is only very mildly concave in lateral view. In general, the ungual is not particularly compressed mediolaterally. The proximal articular surface is mediolaterally flat and dorsoventrally concave; the latter is largely a result of the overhanging dorsal margin of the ungual. A foramen pierces the proximal articular surface, close to the lateral margin just above midheight. The medial surface of the ungual is dorsoventrally convex, whereas the lateral surface is dorsoventrally concave. Vascular foramina are present on both the lateral and medial surfaces.

Phalanx II-2 is a small, incomplete nubbin of bone with a convex distal surface. The distal surface of this element, along with those of phalanges III-1, IV-1 and V-1, indicate that no additional phalanges are missing from MB.R.2093.

In summary, the manus clearly belongs to a eusauropod and, based on the expansion of the distal articular surfaces onto the dorsal surfaces of the metacarpals and bevelled distal end of metacarpal I, it seems unlikely that it represents a titanosauriform. Diplodocoid synapomorphies are not currently known for the manus (Wilson, 2002; Upchurch \& Mannion, 2009; Whitlock, 2011a), and well preserved manual material confidently attributed to non-titanosauriform macronarians is restricted to Camarasaurus (Tschopp et al., 2015b). The low phalangeal formula hints at possible neosauropod affinities, but ultimately our knowledge of manual morphology of taxa around the onset of the neosauropod radiation is poor. Consequently, the manus could conceivably belong to Janenschia, as proposed by Janensch (1922), but to avoid the potential danger of creating a chimeric taxon, it is here regarded as an indeterminate eusauropod.

\section{Quarry G}

Quarry $\mathrm{G}$ is a multitaxic assemblage, with 98 elements originally listed by Janensch from this site (Remes, 2007). As well as material originally referred to Janenschia, including the caudal sequence MB.R.2091.1-30 (now the holotype of Wamweracaudia), sauropod elements from this quarry were also attributed to Dicraeosaurus, Giraffatitan, and Tornieria (Janensch, 1961; Remes, 2007). More recently, two cervical vertebrae were described as a new taxon, Australodocus (Remes, 2007), and a third cervical vertebra (MB.R.2091.31) was tentatively referred to Tendaguria (Bonaparte et al., 2000). Unfortunately, no map of Quarry 
$\mathrm{G}$ has survived (Heinrich, 1999b; Remes, 2007), and most elements are now lost (Remes, 2007), but it is clear from Janensch's notes that skeletal materials were widely distributed and that multiple individuals were present. Of this material, Janensch (1929a) originally referred a cervical vertebra (listed as a dorsal vertebra in his table of materials), caudal vertebrae, and ribs to Janenschia, and an ischium was subsequently also referred (Janensch, 1961). However, only the ischium (MB.R.2091.32 [G77]) could potentially be referred to Janenschia based on existing overlapping elements: no cervical vertebrae have ever been known from quarries $B$ or $P$, and the lost caudal vertebrae from Quarry $\mathrm{P}$ appear to comprise middle-posterior caudal centra (Bonaparte et al., 2000: fig. 3), making recognition of diagnostic features unlikely. Currently, the cervical vertebra (MB.R.2091.31 [G45]) is tentatively referred to Tendaguria, and the caudal vertebrae (MB.R.2091.1-30) comprise the holotype of Wamweracaudia. The ribs could not be located in the MfN collections. Although it is possible that Wamweracaudia is the same taxon or even individual as the elements comprising Australodocus or Tendaguria, and all might be referable to Janenschia, the current lack of overlapping material precludes any comparison (see 'Oversplitting of Tendaguru sauropods?' below). One piece of evidence indicates that they are unlikely to represent the same individual at least: the colour and preservation of all three differs, suggesting that they were from different parts and/or stratigraphic levels of the quarry. Below we discuss the remaining appendicular sauropod material from Quarry $G$ and assess its taxonomic affinities.

The right ischium (MB.R.2091.32) is damaged at its proximal end and along the ventral margin of the proximal portion of the blade (Fig. 36; see Table 14 for measurements). The lack of autapomorphies for the ischium of Janenschia, coupled with the poor preservation of MB.R.2091.32, makes assignment of the latter difficult. However, the lateral ridge for attachment of M. flexor tibialis internus III is very weakly developed in MB.R.2091.32, and appears to be associated with a groove, contrasting with the condition in the topotypic ischia of Janenschia. The blade of MB.R.2091.32 is also orientated at a shallower angle than those of the Janenschia topotypes. As such, there is no reason to refer this ischium to Janenschia, and we assign it to an indeterminate eusauropod.

Most of the additional material listed from Quarry $\mathrm{G}$ is now lost. All that remains are: (1) two humeri originally referred to Tornieria (MB.R.2656 [G81] and 2709 [G?]; see Remes, 2009); (2) a left radius originally referred to Dicraeosaurus (MB.R.2622 [G83]); (3) a right tibia (MB.R.2595 [G82]); (4) and two pedal unguals (MB.R.2321 [G98] and 2332 [G90], the latter originally referred to Giraffatitan). The two humeri display a strong degree of torsion between their proximal and distal ends, and lack the well rounded proximal outline seen in the humerus of Janenschia (MB.R.2095.7). The distal end of the radius (MB.R.2622) is only gently bevelled, and the tibia (MB.R.2595) lacks the autapomorphic tuberosity on the cnemial crest or a 'second cnemial crest'. The pedal unguals of Janenschia lack diagnostic features, precluding the referral of MB.R.2321 or MB.R.2332.

In summary, there is no evidence for the presence of Janenschia in Quarry G based on comparisons with overlapping appendicular elements from quarries $\mathrm{B}$ and $\mathrm{P}$, and thus there is no a priori reason to assume that any of the vertebrae from Quarry $G$ belong to Janenschia.

\section{Quarry IX}

Janensch (1961) referred a right radius and right femur to Janenschia from Quarry IX, which is a multitaxic, disarticulated assemblage situated approximately $1.4 \mathrm{~km}$ northeast of Tendaguru Hill (Janensch, 1961; Heinrich, 1999b; Bonaparte et al., 2000). This site was 
described as situated in the 'Upper Transitional Sands' (Janensch, 1961; Heinrich, 1999b), i.e. at the base of the Tithonian Upper Dinosaur Member (Bussert et al., 2009).

Originally described as a left element (Janensch, 1961; Bonaparte et al., 2000), the right radius (MB.R.2096.2 [IXv3]) is complete (Fig. 37; see Table 15 for measurements). Its proximal end is oval, with a slight medial projection as a result of anteroposterior shortening towards the medial margin. The proximal articular surface is rugose and concave. Three ventrolaterally directed ridges are present along the distal half to two-thirds of the posterior surface. The most laterally situated of these ridges extends along the posterolateral margin and expands anteroposteriorly along the distal third, such that it is visible in anterior view. In anterior view, the distal end is bevelled along the lateral two-thirds. The rugose distal end has an elliptical to subtriangular outline, with its long axis oriented transversely. Although it shares some features with the radius of Janenschia (MB.R.2095.9), including the distal bevelling, there are no shared autapomorphies, and so we assign MB.R.2096.2 to an indeterminate eusauropod.

The right femur (MB.R.2096.1 [IX c1]) is complete (Fig. 38; see Table 15 for measurements), although the proximal third has undergone some distortion. The proximolateral margin, above the lateral bulge, is deflected medially with respect to the lateral margin of the distal half of the shaft. There is no trochanteric shelf, and the fourth trochanter is not visible in anterior view. The distal condyles curve up strongly on to the posterior surface of the femur, but do not extend on to the anterior surface. The central third of the anterior portion of the distal surface slopes to face anteroventrally. The tibial condyle is larger anteroposteriorly than the fibular condyle (relative length ratio $=1.2$ ), whereas this ratio is 1.1 in the holotype (SMNS 12144) and referred (MB.R.2707) femora of Janenschia. In all other regards, MB.R2096.1 does not differ in any substantial way from the femora of Janenschia; however, the lack of clear autapomorphic features of the Janenschia femur precludes referral of MB.R.2096.1, and so we regard it as belonging to an indeterminate eusauropod.

\section{Quarry dd}

Janensch (1925a) mentioned material from Quarry dd that he referred to Janenschia, but did not state what this material comprised, and there is no further mention in subsequent publications describing the Tendaguru dinosaurs (e.g. Janensch, 1961). No elements from Quarry dd labelled as Janenschia could be located in the MfN collections and, as such, we cannot confirm the presence of this taxon at that locality.

\section{Quarry Nr. 22}

Janensch (1961: table 14) listed a left femur from site 'Nr. 22' under measurements for Janenschia, but this element was not mentioned in the text, nor was it figured. It also went unmentioned in Bonaparte et al. (2000) and it could not be located in the MfN collections. It is possible that this is the same element as MB.R.2707 (see 'Description and comparisons of material referable to Janenschia robusta from Quarry P'), which is labelled as P22 and has very similar dimensions to those provided by Janensch (1961). Although it is possible that MB.R.2707 is from an isolated quarry (i.e. ' $\mathrm{Nr} .222^{\prime}$ ), there is no mention of this locality in any other of Janensch's papers and ' $\mathrm{Nr}$ ' is not marked anywhere on the element. Furthermore, a femur of the same length is listed from Quarry $P$ in Janensch (1929a: p. 26). As such, we tentatively accept the provenance of MB.R.2707 as Quarry P.

\section{NHMUK material from the British expeditions}


Numerous undescribed elements collected by the British expeditions to Tendaguru (Migeod, 1931; Maier, 2003) are stored in the NHMUK; however, an examination of this material (PDM pers. obs. 2013) revealed no elements that could be confidently attributed to Australodocus, Janenschia, Tendaguria or Wamweracaudia.

\section{TAXONOMIC AFFINITIES OF MATERIAL REFERRED TO JANENSCHIA FROM THE LATE JURASSIC OF ZIMBABWE}

Raath \& McIntosh (1987) referred numerous fragmentary materials from the Upper Jurassic Kadzi Formation of Zimbabwe to Tendaguru taxa. Amongst these materials are a caudal sequence, humerus, and partial femur referred to Janenschia ('Tornieria' in Raath \& McIntosh 1987). One of the most anterior caudal vertebrae is figured (presumably Cd1-3? based on the possession of a 'wing'-like rib) and is procoelous, although the posterior convexity is much less developed than in Wamweracaudia. Furthermore, as preserved, the ventral margin of the caudal rib is deflected dorsally. As such, it seems likely that this tail represents a flagellicaudatan, and probably a dicraeosaurid, based on the combination of procoely coupled with a dorsally deflected ventral margin of the caudal rib (Mannion et al., 2011; Whitlock, 2011a). The overall morphology of the humerus, including the rounded proximal end, is reminiscent of that of Janenschia, but there is no unambiguous basis for its referral. The femur is proximally and distally incomplete and, like the humerus, its affinities cannot be ascertained beyond Sauropoda. Consequently, there is no unequivocal evidence for the presence of Janenschia or Wamweracaudia in the Late Jurassic of Zimbabwe.

\section{INTERNAL TISSUE STRUCTURE OF AUSTRALODOCUS BOHETII}

Australodocus bohetii is known from only two cervical vertebrae - MB.R.2455 (holotype) and MB.R.2454 (paratype) (Remes, 2007). Although largely complete, both vertebrae are compressed and are broken in several areas.

CT scans reveal that both vertebrae are filled with a dense network of pneumatic structures, which invade the complete centrum, as well as the neural arch (including the zygapophyses) and neural spine (Figs 39, 40). Although several of the thin pneumatic cavity walls have collapsed and the shape of the camellae is partly not preserved, contrast between fossilized bone and sediment infill of the cavities is sufficient to allow separation of these structures. These pneumatic cavities are entirely formed by angular camellae, which have straight and very thin bone walls (Figs 39A-C, 40A, B). The camellae continue in a network of equally large or only slightly larger succeeding chambers (Figs $39 \mathrm{M}, 40 \mathrm{~K}, \mathrm{~L}$ ), but none of the pneumatic hollows branches out into significantly larger chambers. The condyle is filled by a regular pattern of similarly small camellae that expand into slightly larger and more or less radially ordered camellae at the transition to the main body of the centrum. MB.R.2455 has fewer, but larger camellae in this region than MB.R.2454. In the condyle of MB.R.2455, the bone walls are internally hollowed out by very small pneumatic cavities (Fig. 39B, M). The parapophyses are heavily pneumatised with small camellae.

At the level of the diapophyseal table, the vertebral body of MB.R.2454 has a massive centre, to which some isolated pneumatic camellae are positioned ventrally. Dorsal to this massive centre, a zone of equally large and regular camellae encircles the neural canal and invades most of the area of the vertebra, leaving only thin bone walls. Camellae around the neural canal are partly connected to the latter via small canals. The floor of the neural canal is underlain by a concentration of small, rounded camellae (Figs 39E, 40F). Posterior to the 
diapophyses, the vertebral body is reduced to a median, narrow strut of bone, which is itself further hollowed out by camellae (Figs 39F-I, 40E, F). The vertebral body is more massive in its posterior half at the neural arch, and completely hollowed out by a regular pattern of pneumatic camellae twice as large as those in the vertebral condyle.

The prezygapophyses are hollowed out by regular camellae, which expand within the SPRL into a larger, tubular pneumatic chamber divided medially by a narrow septum (Figs $39 \mathrm{C}-\mathrm{E}, 40 \mathrm{C}-\mathrm{E}$ ). On each side of the vertebral body, these tubular chambers are retained until the ends of the diapophyses, and form a pneumatic camera within them. The diapophyses are also hollowed out by small camellae, and house the large CDF beneath them. The postzygapophyses are completely hollowed out by small camellae, as is the bifid neural spine. Towards the apex of the neural spine, the camellae become smaller and rounded, and are surrounded by more massive bone walls (Figs 39G-I, 40G, H).

The CT sections confirm the observations of Whitlock (2011b; based on broken outer surfaces) that both vertebrae have an irregular branching pattern of $<20 \mathrm{~mm}$, angular, intravertebral pneumatic camellae, forming a 'honey-comb' internal pattern. This pneumatization pattern corresponds to a fully camellate or somphosphondylous pneumatization type (Wedel et al., 2000; Wedel, 2003, 2005), invading the centrum, neural arch, zygapophyses, and neural spine. The somphospondylous pattern found in the cervical vertebrae of Australodocus is more developed than in basal titanosauriforms such as Giraffatitan (Schwarz \& Fritsch, 2006) or Sauroposeidon (Wedel et al., 2000), and is morphologically closer to the condition seen in derived titanosaurs such as Saltasaurus (Powell, 1992; Wedel, 2003; Cerda et al., 2012), Neuquensaurus (Cerda et al., 2012), and possibly Alamosaurus (Woodward \& Lehman, 2009). This supports the re-interpretation of Whitlock (2011b) that Australodocus is a titanosauriform, rather than a diplodocid, as originally proposed by Remes (2007). Furthermore, it provides additional support for a somphospondylan placement within Titanosauriformes (see also the Discussion), as recovered in the analyses of Mannion et al. (2013).

\section{PHYLOGENETIC ANALYSIS OF TENDAGURU SAUROPODS}

\section{DATA SET}

Whereas there is consensus regarding the flagellicaudatan placements of Dicraeosaurus and Tornieria (e.g. Upchurch et al., 2004; Remes, 2006; Whitlock, 2011a), as well as the brachiosaurid affinities of Giraffatitan (e.g. Taylor, 2009; D'Emic, 2012; Mannion et al., 2013), the phylogenetic positions of the remaining four Tendaguru sauropod taxa (Australodocus, Janenschia, Tendaguria, Wamweracaudia) have proven more problematic and controversial (see Introduction). We investigated their positions via an updated version of the only data matrix to already include all of these taxa (Mannion et al., 2013; revised by Poropat et al., 2015a, b, 2016; Upchurch et al., 2015; Mannion et al., 2017; González Riga et al., 2018). With the exception of modifications to characters, none of the existing scores for these four Tendaguru taxa have been revised following our study, although we restricted Tendaguria to its holotypic dorsal vertebrae. To thoroughly test the placements of these enigmatic taxa, we added 33 taxa and 118 characters to the most recent iteration of this matrix (González Riga et al., 2018), as well as revising existing characters and scores. In particular, we focused on diplodocoids, as well as taxa generally recovered close to Neosauropoda, as documented below. 
Both Dicraeosaurus and Tornieria were added so that all seven named Tendaguru sauropod genera are included in our data matrix. Scores for Dicraeosaurus, the clade specifier for Dicraeosauridae, were based on Janensch $(1929,1935-36,1961)$ and SchwarzWings \& Böhm (2014), whilst the diplodocine diplodocid Tornieria was scored following Janensch (1935-36) and Remes (2006, 2007, 2009). Information on both taxa was supplemented via personal observations (MfN MB.R. and SMNS specimens) by PU (1992) and PDM (2014).

Lingwulong shenqi is a dicraeosaurid diplodocoid from either the late Early or early Middle Jurassic Yanan Formation of China, and is the stratigraphically oldest neosauropod (Xu et al., 2018). It was scored based on Xu et al. (2018) and personal observations by PDM and PU (LM V001a, LGP V001-006, and IVPP V23704, in 2016). The late Middle Jurassic UK sauropod Cetiosauriscus stewarti, from the Oxford Clay Formation (Woodward, 1905), has been included in only a small number of phylogenetic analyses, but has been recovered either as a 'basal' diplodocoid (e.g. Tschopp et al., 2015a), or non-neosauropod eusauropod, with possibly close affinities to Omeisaurus and Mamenchisaurus (Rauhut et al., 2005). Our scores for Cetiosauriscus stewarti are based on firsthand observation of the type specimen (NHMUK R3078) by PDM and PU $(2012,2017)$. This excludes the series of distal caudal vertebrae (NHMUK R1967) that were erroneousaly referred by Woodward (1905) (see Upchurch et al., 2004; Naish \& Martill, 2007). The Middle Jurassic Chinese mamenchisaurid Chuanjiesaurus anaensis, from the Chuanjie Formation, was scored based on Sekiya (2011). Bellusaurus sui, from the late Middle Jurassic/early Late Jurassic section of the Shishugou Formation of China (Dong, 1990), has been recovered as a non-neosauropod eusauropod (e.g. Wilson \& Upchurch, 2009; Royo-Torres \& Upchurch, 2012; Mo, 2013) or 'basal' macronarian (e.g. Upchurch et al., 2004; Carballido et al., 2015; Moore et al., 2015) in previous analyses. Our scores for this taxon are based on Mo (2013), personal observations (IVPP specimens) by PU $(2007,2010)$ and PDM (2012), as well as photographs provided by JL Carballido.

The Late Jurassic sauropod Haplocanthosaurus priscus, from the Morrison Formation of the USA, has been recovered as a non-neosauropod eusauropod, a 'basal' diplodocoid, and a non-titanosauriform macronarian in different analyses (see Mannion et al. [2012] for a summary). It was scored using CM 572 and CM 879 (PDM pers. obs. 2013), as well as information provided in Hatcher (1903). Other remains previously referred to Haplocanthosaurus priscus (McIntosh, 1981) cannot currently be confidently attributed to this taxon. A second, contemporaneous species, Haplocanthosaurus delfsi, was named by McIntosh \& Williams (1988), but its affinities have received little attention. In the rare instances in which it has been included in a phylogenetic analysis, Haplocanthosaurus delfsi has been recovered as either a 'basal' diplodocoid (Calvo \& Salgado, 1995) or a nontitanosauriform macronarian (Gallina \& Apesteguía, 2005). In neither analysis were the two Haplocanthosaurus species recovered as sister taxa. Our scores for Haplocanthosaurus delfsi are based on McIntosh \& Williams (1988) and firsthand observations of the type specimen CMNH 10380 (PU \& PDM, 2008). Amphicoelias altus, also from the Morrison Formation, has been regarded as either a 'basal' diplodocoid (Whitlock, 2011a) or diplodocid (Tschopp et al., 2015a; Tschopp \& Mateus, 2017). It was scored here based on the type specimen AMNH 5764 (PDM pers. obs. 2015; note that this excludes the tooth, scapula, coracoid, and ulna [see Whitlock, 2011a; Mannion et al., 2012]) and information presented in Osborn \& Mook (1921). Lourinhasaurus alenquerensis, from the Late Jurassic Lourinhã Formation of Portugal, is generally considered to be a non-titanosauriform macronarian (Mocho et al., 2014). Its OTU was restricted to the lectotype material collected from the type locality, and our scores 
are based on Mocho et al. (2014) and personal observations by PDM and PU (MG specimens in 2009).

Early analyses of the affinities of Suuwassea emilieae, from the US Morrison Formation, supported a 'basal' diplodocid placement (Gallina \& Apesteguía, 2005; Rauhut et al., 2005; Remes, 2006; Lovelace et al., 2008), but all recent studies have recovered it as the sister taxon of all other dicraeosaurids (Salgado et al., 2006; Whitlock, 2011a; Carballido et al., 2012; Mannion et al., 2012; Tschopp et al., 2015a; Xu et al., 2018). Our scores for Suuwassea are based on Harris \& Dodson (2004), Harris (2006a,b,c, 2007), Whitlock \& Harris (2010), and Whitlock (2011a). We also included the Argentinean dicraeosaurids Brachytrachelopan mesai and Amargasaurus cazaui from the Late Jurassic Cañadón Calcáreo Formation and Early Cretaceous La Amarga Formation, respectively. Brachytrachelopan was scored based on firsthand observations (MPEF PV 1716: PDM \& PU, 2013), as well as information in Rauhut et al. (2005). Scores for Amargasaurus were also based on firsthand study (MACN N-15: PDM \& PU, 2013), supplemented by information presented in Salgado \& Bonaparte (1991), Salgado \& Calvo (1992), and Paulina Carabajal (2012).

The Morrison Formation diplodocine Supersaurus vivianae was scored following Jensen (1985), Curtice \& Stadtman (2001), and Lovelace et al. (2008), augmented by firsthand observations of the referred specimen WDC DMJ-021 (Lovelace et al., 2008) by PDM (2008). Dinheirosaurus lourinhanensis, from the Late Jurassic Lourinhã Formation of Portugal (Bonaparte \& Mateus, 1999), has been recovered as the sister taxon to Supersaurus in recent phylogenetic analyses (Mannion et al., 2012), with Tschopp et al. (2015a) recombining it as a second species of the latter genus. Our scores are based on Bonaparte \& Mateus (1999), Mannion et al. (2012), and firsthand study of the type specimen ML 414 (PDM \& PU, 2009). Mannion et al. (2012) stated that Dinheirosaurus definitely possesses unbifurcated posterior cervical neural spines. However, re-examination of the type specimen (PDM pers. obs. 2012) suggests that it is not clear which state is present, and thus this feature is coded as a "?" here. We also included the earliest Cretaceous diplodocine Leinkupal laticauda, from the Bajada Colorada Formation of Argentina, following information presented in Gallina et al. (2014).

All named rebbachisaurid taxa were incorporated (note that Nigersaurus was already present in earlier versions of the data matrix), as well as two currently unnamed specimens that provide additional anatomical information. The late Early Cretaceous Brazilian taxon Amazonsaurus maranhensis (Carvalho, Avilla \& Salgado, 2003), from the Itapecuru Formation, has been recovered in a range of placements, including a basal flagellicaudatan (Salgado et al., 2004), basal macronarian (Rauhut et al., 2005), and basal diplodocoid (Whitlock, 2011a) position. However, other authors have found it to fall within Rebbachisauridae (Salgado et al., 2006; Carballido et al., 2010), where it has generally been recovered as the sister taxon of all other members of this clade (e.g. Carballido et al., 2012; Mannion et al., 2012; Wilson \& Allain, 2015). Our scores for Amazonsaurus are based on Carvalho et al. (2003) and personal observations by PDM (MN 4555-4564 and UFRJ-DG 58 in 2009). Scores for the Early Cretaceous Croatian taxon Histriasaurus boscarollii (unnamed stratigraphic unit) follow information provided in Dalla Vecchia $(1998,1999,2005)$. As a result of the present day location of Croatia, Histriasaurus has tended to be considered as a European taxon by many authors (Sereno et al., 2007; Carballido et al., 2010, 2012; Whitlock, 2011a; Mannion et al., 2012; Fanti et al., 2013, 2015). However, as detailed by Dalla Vecchia (2005), this region was actually a fragment of Gondwana during the Early Cretaceous, and thus the provenance of Histriasaurus should be more accurately regarded as Afro-Arabian. The late Early Cretaceous Argentinean rebbachisaurids Comahuesaurus 
windhauseni (Lohan Cura Formation) and Zapalasaurus bonapartei (La Amarga Formation) are scored based on personal observations by PDM (MOZ-PV specimens in 2009), as well as information presented in Salgado et al. $(2004,2006)$ and Carballido et al. (2012).

The rebbachisaurids Cathartesaura anaerobica (Huincul Formation), Katepensaurus goicoecheai (Bajo Barreal Formation), Limaysaurus tessonei, Nopcsaspondylus alarconensis, Rayososaurus agrioensis (last three all from the Candeleros Formation), and an unnamed specimen from the Huincul Formation briefly described by Haluza et al. (2012), all come from the early Late Cretaceous of Argentina. Cathartesaura is scored based on Gallina \& Apesteguía (2005) and personal observations by PDM (MPCA 232, in 2009), supplemented by photographs provided by JA Whitlock \& V Zurriaguz. Scores for Katepensaurus follow firsthand observations of the type specimen (UNPSJB-PV 1007) by PDM \& PU (2013), along with information provided in Ibiricu et al. (2013, 2015). Limaysaurus is scored based on firsthand study (MUCPv-205 and referred material accessioned under MUCPv), as well as information presented in Calvo \& Salgado (1995), supplemented by photographs provided by JA Whitlock. The sole specimen of Nopcsaspondylus is unfortunately lost (Apesteguía, 2007), but was scored based on the figures provided in Nopcsa (1902). Scores for Rayososaurus follow Carballido et al. (2010) and personal observations of the type specimen (MACN PV-N41) by PDM $(2009,2014)$. The aforementioned unnamed rebbachisaurid (herein referred to as the 'El Chocon rebbachisaurid') is scored based on the information presented by Apesteguía et al. (2010) and Haluza et al. (2012), supplemented by firsthand study of the figured remains (MMCH PV 49: PDM pers. obs. 2009).

Demandasaurus darwini is a rebbachisaurid from the late Early Cretaceous Castrillo de la Reina Formation of Spain and is scored based on Pereda Suberbiola et al. (2003), Torcida Fernández-Baldor et al. (2011), Torcida Fernández-Baldor (2012), and firsthand study (MDSRVII: PDM \& PU pers. obs., 2009). A partial caudal vertebra from the contemporaneous Wessex Formation in the UK shows a number of similarities to Demandasaurus and is included here as an OTU following Mannion et al. (2011a) and personal observations of the specimen (MIWG 5384) in 2010 (PDM \& PU). It is herein referred to as the 'IOW rebbachisaurid caudal'. The Tunisian rebbachisaurid Tataouinea hannibalis is also from the late Early Cretaceous (Aïn el Guettar Formation) and was scored based on Fanti et al. (2013, 2015). Scores for Rebbachisaurus garasbae, from the early Late Cretaceous Kem Kem Beds of Morocco, follow Wilson \& Allain (2015), supplemented by firsthand study of the type material by PDM (MNHN MRS 1958 and additional MNHN MRS specimens) in 2011 and 2014.

Moabosaurus utahensis is a eusauropod from the late Early Cretaceous Cedar Mountain Formation of the USA, that Britt et al. (2017) suggested shared affinities with some of the Tendaguru taxa. Royo-Torres et al. (2017a) scored Moabosaurus for an earlier iteration of this matrix (Mannion et al., 2017), although many of these scores are revised based on Britt et al. (2017) and personal observations by PU (BYU specimens in 2013). A second taxon (Mierasaurus bobyoungi) from the same formation was described by RoyoTorres et al. (2017a) and was also included in the Mannion et al. (2017) matrix. Both Mierasaurus and Moabosaurus were recovered as turiasaurs by Royo-Torres et al. (2017a). Our scores for Mierasaurus follow those authors, although many of these are revised based on personal observations by PU (UMNH.VP.26004 in 2013). Finally, Royo-Torres et al. (2017b) described a new brachiosaurid, Soriatitan golmayensis, from the Early Cretaceous Golmayo Formation of Spain, and included this in the matrix of Mannion et al. (2017). Our scores for Soriatitan are based on Royo-Torres et al. (2017b), although we have made a small number of changes to the existing scores (see Appendix 1). 
Royo-Torres et al. (2017a) also proposed a number of changes to existing scores in the Mannion et al. (2017) matrix. They revised scores of three dental characters for the late Middle Jurassic Moroccan non-neosauropod eusauropod Atlasasaurus imelakei (Guettioua Formation), based on Monbaron et al. (1999). However, we reject all three changes: two (C105 and C110) cannot be confidently determined from information provided in Monbaron et al. (1999) and are retained as missing data here. The third character (C108) pertains to the morphology of tooth crowns in labial view. Royo-Torres et al. (2017a) revised the score of Mannion et al. (2017) from the derived state (little expansion of the crown above the root) to the plesiomorphic condition (constricted at the base relative to midheight of the crown), but there is no evidence for any constriction at the base (Monbaron et al., 1999: fig. 1f, g) and so we retain the original score. Several scores were also changed for the latest Jurassic/earliest Cretaceous Spanish turiasaurs Losillasaurus and Turiasaurus (both from the Villar del Arzobispo Formation). We follow many of these recommendations, but disagree with some. For example, these authors scored the maxilla of Turiasaurus as possessing a preantorbital fenestra (see Royo-Torres \& Upchurch, 2012), but the relevant region of this element does not appear to be preserved (PU pers. obs. 2010). Royo-Torres et al. (2017a) also scored Losillasaurus for features pertaining to posteriormost cervical-anteriormost dorsal neural spines and the proximal ulna, but these regions of the skeleton are not preserved (Casanovas, Santafe \& Sanz, 2001; PDM \& PU pers. obs. 2009). Although other remains have been referred to Turiasaurus (Royo-Torres et al., 2009), their referral was based on shared plesiomorphies, rather than clear autapomorphies. As such, we continue to restrict Turiasaurus to the type individual, pending unambiguous referral of new material. Royo-Torres et al. (2017b) also proposed a number of changes to the scores of the late Early Cretaceous Spanish somphospondylan Tastavinsaurus (Xert Formation), and we follow most of these recommendations. We also made a small number of changes to our scores for two non-titanosaurian somphospondylan OTUs ('Paluxysaurus' and the 'Cloverly titanosauriform') based on a pers. comm. and photographs from MD D'Emic (2016).

Although this part of the tree was not the focus of our study, we also augmented scores for the latest Cretaceous Mongolian titanosaur Nemegtosaurus (Nemegt Formation) based on newly discovered postcranial remains belonging to the type individual, recently described by Currie et al. (2018). As part of that study, those authors briefly described a fragmentary partial anterior caudal centrum, which is convex at one end. They interpreted this centrum as opisthocoelous, a feature otherwise known only in the contemporaneous titanosaur Opisthocoelicaudia. However, Currie et al. (2018) presented no information to support their interpretation that the convexity is anterior rather than posterior (i.e. procoely: the condition in nearly all other titanosaurs); as such, we err on the side of caution and have not scored Nemegtosaurus for characters pertaining to either opisthocoely or procoely of anterior caudal centra.

We also revised 21 existing character definitions and added in 118 characters. The latter are primarily modified from previous authors (e.g. Wilson, 2002; Whitlock, 2011a; Tschopp et al., 2015a), supplemented by novel characters emanating from a thorough review of the literature and our personal observations. Our final data set comprises 117 taxa scored for 542 characters, which is the largest data set for sauropods, both in terms of numbers of taxa and characters. Of these 117 taxa, 83 have been studied firsthand by at least one of the two lead authors of the current work. All changes to existing characters and scores are documented in Appendix 1, and the complete character list is provided in Appendix 2. We also provide our full data matrix as both a Mesquite and TNT file (Supporting Information S1 and S2). 


\section{ANALYTICAL PROTOCOL AND RESULTS}

Characters 11, 14, 15, 27, 40, 51, 104, 122, 147, 148, 195, 205, 259, 297, 426, 435, 472, and 510 were treated as ordered multistate characters. Following previous versions of this data matrix, and preliminary analyses, several fragmentary and unstable taxa were identified (Astrophocaudia, Australodocus, Brontomerus, Fukuititan, Fusuisaurus, Liubangosaurus, Malarguesaurus). With the exception of the Tendaguru sauropod Australodocus, these unstable taxa were excluded a priori. Using equal weighting of characters, the pruned data matrix was analysed using the 'Stabilize Consensus' option in the 'New Technology Search' in TNT vs 1.5 (Goloboff, Farris \& Nixon, 2008; Goloboff \& Catalano, 2016). Searches were carried out employing sectorial searches, drift and tree fusing, with the consensus stabilized five times, prior to using the resultant trees as the starting trees for a 'Traditional Search' utilising Tree Bisection-Reconstruction. This produced 365,013 MPTs of length 2556 steps, with limited resolution in many parts of the tree, although Australodocus was recovered within Somphospondyli, as a member of Euhelopodidae. We then repeated this analytical protocol with Australodocus also excluded. This results in 22,704 MPTs of length 2551 steps, and a relatively well resolved strict consensus (with no results contrasting with the previous analysis), with the exception of a large polytomy within Titanosauria (Fig. 41). Bremer supports are low for most nodes (values of 1 or 2 ).

Finally, we ran the analysis using extended implied weighting (Goloboff, 2014), with the default settings in TNT. We excluded the six unstable taxa listed above a priori, retaining Australodocus. This produced 234 MPTs of length 237.2 steps, and a well resolved strict consensus, with the exception of a large polytomy within Brachiosauridae (including Australodocus). Excluding Australodocus a priori too, this polytomy is partly resolved, and our analysis results in 27 MPTs of length 237 steps (Fig. 42).

\section{BIOGEOGRAPHIC ANALYSIS}

\section{TAXON AGES AND RELATIONSHIPS}

We analysed the biogeographic history of eusauropods, using the $\mathrm{R}$ package BioGeoBEARS (Matzke, 2013, 2014) to estimate the areas occupied at ancestral nodes (see Supporting Information S3 for R script). Such analyses require a fully resolved and dated phylogenetic topology. We therefore constructed agreement subtrees from our equal weights parsimony (EWP) and extended implied weights (EIW) analyses, resulting in trees containing 95 and 99 taxa respectively. These taxa were dated using the Paleobiology Database (https://paleobiodb.org/), with modifications based on the primary literature. The ages of several Jurassic stratigraphic units from China are in a state of flux (e.g. Xing et al., 2015); for example, Wang et al. (2018) recently dated the Shaximiao (=Dashanpu) Formation as Oxfordian, rather than the late Middle Jurassic (Bathonian-Callovian) age that has been the consensus for some time. Although our taxon ages generally represent the latest literature, in this case our ages differ from Wang et al. (2018); however, this only potentially affects our outgroup taxon Shunosaurus (and the second most 'basal' taxon, Omeisaurus), and thus has no impact upon our biogeographic results. Taxa (e.g. Apatosaurus, Camarasaurus) known from multiple specimens, at several stratigraphic horizons, were given true stratigraphic ranges and so have different First Appearance Datum (FAD) and Last Appearance Datum (LAD) values. However, most taxa are known only from a single 
specimen (or several specimens from a single horizon), so that their 'age range' reflects a lack of temporal resolution rather than a true stratigraphic range. These taxa were given midpoint ages, as in other recent studies (e.g. Loewen et al., 2013; Poropat et al., 2016; Xu et al., 2018). All taxon ages were converted into absolute ages, using the 2017 version of the International Commission on Stratigraphy Chronostratigraphic Chart (Cohen et al., 2013), and are provided in Supporting Information files S4 (EWP) and S5 (EIW).

The EWP and EIW trees were calibrated against time using the R package strap (Bell \& Lloyd, 2015), via the DatePhylo command. The root length was set at 5 million years, and adjacent zero-length branches were distributed using the 'equal' method (a modified version of the approach proposed by Brusatte et al. [2008]). The time-calibrated phylogenies (in newick format) are presented in Supporting Information files S6 (EWP) and S7 (EIW) and are shown in Figures 43 and 44.

\section{TAXON GEOGRAPHIC RANGES}

BioGeoBEARS requires a geographic range file. Here, we assign each taxon to one or more of eight continental areas: A, Asia; E, Europe; F, Africa; I, India; M, Madagascar; N, North America; S, South America; and U, Australia. In previous applications of BioGeoBEARS to dinosaurs (e.g. Gorscak \& O'Connor, 2016; Poropat et al., 2016; Xu et al., 2018), taxa are assigned ' 0 ' for all areas where they have not been found to date. However, some of these absences might be the result of inadequate sampling (see also Turner, Smith \& Callery [2009] for discussion of sampling in palaeobiogeographic analysis). Here, therefore, we have developed some 'rules' that specify when a taxon should be assigned ' 0 ' or '?' in the geographic ranges files. These rules are:

1. Taxa of Middle Jurassic age are assigned '?' for North America. This is because of the virtually non-existent terrestrial fossil record from that continent at that time (Weishampel et al., 2004; Peters \& Heim, 2010).

2. Taxa of Early Cretaceous age are assigned '?' for India and Madagascar. This is because there are currently no known dinosaur-bearing deposits from these areas for the Early Cretaceous (Weishampel et al., 2004).

3. Taxa are scored with '?' for Australia except when they are of Middle Jurassic or AptianTuronian age. This is because there are no dinosaur-bearing deposits from Australia for the Late Triassic-Early Jurassic, Late Jurassic, Berriasian-Barremian, or latest Cretaceous (Nair \& Salisbury, 2012; Poropat et al., 2016).

Of course, other more or less stringent rules could be developed. However, we view the three rules outlined above as relatively conservative, being established on well-documented and profound spatiotemporal gaps in the dinosaurian fossil record. The geographic ranges files are presented in Supporting Information files S8 (EWP) and S9 (EIW).

\section{DISPERSAL MULTIPLIER MATRICES}

BioGeoBEARS analysis can be applied solely to a time-calibrated tree and geographic range file; however, this would omit information on the relative positions and connectedness of palaeogeographic regions. The addition of such data is likely to have a positive impact on ancestral area estimation, especially when dealing with large-bodied terrestrial taxa that are unlikely to have dispersed across oceanic barriers (e.g. see 
discussions in Poropat et al. [2016] and Xu et al. [2018]). We have therefore employed the dispersal multiplier matrices used by $X u$ et al. (2018). These matrices control the probability of dispersing between any two of the eight available areas. Since palaeogeography changes through time, Xu et al. (2018) used 25 different dispersal multiplier matrices, one for each of a series of time slices ranging from the Late Triassic to the end of the Cretaceous. These sets of matrices have been constructed in two forms, known as 'harsh' and 'relaxed' (see Poropat et al., 2016). Essentially, the harsh and relaxed versions are identical with respect to well established and generally agreed palaeogeographic relationships, but differ when there is uncertainty in the sequence and timing of connection/disconnection events. When such uncertainty occurs, the harsh matrices assume that dispersal between two areas was very improbable, whereas in the relaxed version the two affected areas are considered to be in contact. In this way, the sensitivity of the biogeographic results to palaeogeographic uncertainty can be assessed (Poropat et al., 2016; Xu et al., 2018). The relaxed and harsh dispersal multiplier matrices are presented in Supporting Information files S10 and S11, respectively, and the time period file (which specifies the starting dates for each of the 25 time slices) is given in Supporting Information file S12 (see Poropat et al. [2016] for an extended and more detailed discussion of the assumptions underpinning the construction of dispersal multiplier matrices for dinosaurs).

\section{BIOGEOBEARS: METHODS AND ASSUMPTIONS}

BioGeoBEARS uses Maximum Likelihood to estimate the areas occupied by the ancestors at nodes in phylogenetic trees (Matzke, 2013, 2014). This implements six models of how the geographic ranges occupied by ancestors and lineages might evolve on a tree: DEC, DEC+J, DIVALIKE, DIVALIKE+J, BAYAREALIKE, and BAYAREALIKE+J. DEC and DIVALIKE allow different forms of vicariance to occur at nodes, whereas BAYAREALIKE does not allow vicariance, and instead constrains daughter lineages to inherit the ranges of their immediate ancestor (Matzke, 2013). The $+\mathrm{J}$ versions of each model have the same rules as their non $+\mathrm{J}$ versions, except that the former allow founder-event speciation, i.e. long-distance geodispersal (Matzke, 2013, 2014). Log likelihood ratio tests and AIC values were then used to identify which of these models best fits the data. We allowed ancestors to occupy up to the full eight geographic areas available. We ran four analyses, using the relaxed and harsh versions of our dispersal multiplier matrices applied to the dated EWP and EIW agreement subtrees. BioGeoBEARS was run in R version 3.2.3 (R Core Development Team, 2015).

\section{BIOGEOGRAPHIC ANALYSES AND RESULTS}

The results of the log likelihood ratio tests and AIC values are presented in Tables 16 (EWP) and 17 (EIW). In all four analyses, the log likelihood ratio tests demonstrate that the $+J$ versions of the biogeographic models are very strongly significantly better fits to the data than are the non $+J$ versions ( $p$-values range from 0.0001 to $3.1 \mathrm{e}^{-20}$; Tables 16,17 ). Moreover, the AIC values for BAYAREALIKE+J are 107.6 (EWP relaxed), 85.6 (EWP harsh), 105.7 (EIW relaxed), and 131.7 (EIW harsh) units lower than the next best supported model (i.e. DEC+J), which suggests that the BAYAREALIKE $+J$ model can be considered to outperform the other five models very strongly (Burnham \& Anderson, 2002). These results indicate that the biogeographic history of the sauropods in this study is best explained in terms of a mix of sympatry, early occurrences of widespread ancestral stocks followed by regional extinction, and founder-event speciation. The lack of support for DEC, DIVALIKE, etc., also means that 
the data provide no clear evidence for continent-scale vicariance (see also Poropat et al., 2016 and Xu et al., 2018). This might reflect the true biogeographic processes controlling the distributions of sauropods, but it is also possible that sampling biases, incorrect phylogenetic topology, and/or errors in the dating of cladogenetic and palaeogeographic events, have obscured any vicariance signal (e.g. see discussion in Upchurch [2008] concerning the special requirements for detecting vicariance, and Poropat et al. [2016] regarding interpretation of BioGeoBEARS results and sampling issues). The ancestral area estimations for the best supported models, i.e. BAYAREALIKE+J for the EWP relaxed, EWP harsh, EIW relaxed, and EIW harsh, are shown in Supporting Information files S13, S14, S15, and S16, respectively.

\section{DISCUSSION}

\section{PHYLOGENETIC AFFINITIES OF TENDAGURU TAXA}

\section{Janenschia}

Mannion et al. (2013) recovered Janenschia as a non-titanosauriform macronarian, and this position was retained in subsequent revisions of this dataset, where it formed a clade with Haestasaurus, Tehuelchesaurus, and Camarasaurus. This placement at the base of Macronaria was also supported in the analysis of Carballido et al. (2011), although their Janenschia OTU included the caudal sequence (Wamweracaudia) and the Nr. 5 manus, neither of which can be confidently referred to Janenschia (see above). D'Emic (2012) tentatively suggested non-somphospondylan titanosauriform affinities for Janenschia, with a position within Titanosauriformes supported by the absence of a raised medial triangular scar on the proximal end of the fibula. However, this feature is present in the holotype fibula, but the relevant region is poorly preserved. Here, we recover Janenschia as a nonneosauropod eusauropod. It is placed as the sister taxon to Haestasaurus, from the earliest Cretaceous Hastings Beds Group of the UK (Upchurch et al., 2015), in both sets of analyses. Features uniting these two taxa include a mediolaterally expanded distal humerus, and a robust ulna with a prominent olecranon process. Both sets of analysis also place the Middle/Late Jurassic Chinese sauropod Bellusaurus in this clade. In our EWP analysis, these three taxa form a clade with the Late Jurassic Argentinean sauropod Tehuelchesaurus (Cañadón Calcáreo Formation), with this grouping forming the sister taxon to all other turiasaurs. However, in our EIW analysis, these three taxa are more closely related to Neosauropoda than Turiasauria, and Tehuelchesaurus is recovered as a non-titanosauriform macronarian. The latter result is consistent with most recent analyses (e.g. Carballido et al., 2011; D’Emic, 2012; Mannion et al., 2013).

\section{Tendaguria}

Tendaguria was positioned as the sister taxon to Neosauropoda or as a basal diplodocoid in the analyses of Mannion et al. (2013), and as a non-neosauropod or nontitanosauriform macronarian by Carballido et al. (2011). In contrast, Carballido et al. (2012) recovered Tendaguria as a somphospondylan (see also Carballido \& Sander, 2014), although Upchurch et al. (2015) demonstrated that this was the most unstable taxon in that data matrix. Both sets of analyses of the updated data matrix employed here place Tendaguria in a novel position, as a member of the non-neosauropod eusauropod clade Turiasauria, forming a clade with the Early Cretaceous North American taxa Moabosaurus (its sister taxon) and Mierasaurus. Tendaguria and Moabosaurus are united based on the presence of the following features in their anterior dorsal vertebrae: (1) a fossa on the anterior surface 
of the diapophysis, close to the distal end; (2) prominent epipophyses; and (3) an extremely low, non-bifid neural spine.

Bonaparte et al. (2000) also erected a new family to include Tendaguria, the monogeneric Tendaguriidae. However, this has largely been ignored by subsequent authors, with monogeneric higher taxa often regarded as being of little utility in taxonomic or systematic work (Upchurch et al., 2004). A case could be made to re-rank Tendaguriidae as a clade uniting Tendaguria with Moabosaurus and Mierasaurus, to the exclusion of the European turiasaurs, but we prefer to wait until the relationships proposed herein are tested with new data and further analysis. As such, we recommend the continued disuse of Tendaguriidae.

\section{Australodocus}

Australodocus was recovered as a titanosaur in the analyses of Mannion et al. (2013), but the revised matrices presented in Poropat et al. (2015a, b, 2016) resulted in a placement outside of Titanosauria, as a basal somphospondylan. Both sets of analyses presented here support a non-titanosaurian titanosauriform placement; however, whereas our EWP analysis recovers Australodocus as a somphospondylan (within Euhelopodidae), our EWI analysis places it within Brachiosauridae (Figs 41, 42). As such, a titanosauriform placement appears secure for Australodocus (Whitlock, 2011b), and it clearly does not represent a diplodocid (contra Remes, 2007), but its precise affinities within Titanosauriformes cannot currently be confirmed. Based on the extent and nature of the camellae (not fully captured in existing phylogenetic characters), we argue that a somphospondylan identification is the most likely.

We are cautious about the likelihood of Australodocus representing a euhelopodid. This relationship is primarily driven by these taxa all possessing bifurcated cervical neural spines, but this feature has evolved independently in several sauropod lineages (e.g. Wilson, 2002; Upchurch et al., 2004). Furthermore, there are currently no unambiguous occurrences of Euhelopodidae outside of East Asia (see D'Emic, 2012; Mannion et al., 2013). However, given that Euhelopodidae is here recovered as the most basal clade within Somphospondyli, and that the Mengyin Formation (which yielded the clade specifier Euhelopus) is now thought to be earliest Cretaceous (Berriasian-Valanginian) in age (Xu \& Li, 2015), a close relationship between Australodocus and this clade might not be so unlikely. Furthermore, teeth from the Barremian of Spain possess a morphology that is otherwise only known in East Asian Cretaceous sauropods (Canudo et al., 2002), including Euhelopus (Wilson \& Upchurch, 2009). Coupled with our recovered position for Australodocus, these teeth hint at the possibility that Euhelopodidae was more widespread, but could alternatively indicate that Australodocus and Euhelopodidae both retain the plesiomorphic somphospondylan condition. Finally, regardless of which of these positions for Australodocus within Titanosauriformes is correct, our analyses indicate that it is not a titanosaur: as such, there is no unambiguous evidence for the pre-Cretaceous presence of this clade (D'Emic, 2012).

\section{Wamweracaudia}

In the analysis of Mannion et al. (2013), Wamweracaudia was placed as a mamenchisaurid. This position is supported here in both sets of analyses, in which it is recovered as the sister taxon of Mamenchisaurus, forming a clade that also contains Chuanjiesaurus. Features uniting Wamweracaudia with these mamenchisaurid taxa include the strong degree of procoely in anterior caudal centra, and the doubling of middle caudal neural spine length from base to summit. 


\section{OVERSPLITTING OF TENDAGURU SAUROPODS?}

Dicraeosaurus, Giraffatitan and Tornieria are each known from abundant material from across much of the skeleton, facilitating comparisons with other taxa. Although Australodocus, Janenschia, Tendaguria and Wamweracaudia are clearly distinct from Dicraeosaurus, Giraffatitan and Tornieria, it is much harder to compare them to one another, leading to the possibility of taxonomic oversplitting. Australodocus and the cervical vertebra tentatively referred to Tendaguria are based on overlapping elements and can easily be distinguished from one another, but no other direct comparisons between taxa can be made. Based on its recovered position as a titanosauriform, with clear differences to Giraffatitan, it seems likely that Australodocus represents a distinct taxon.

Although they are not found to be closely related to one another, the other three taxa are all recovered as non-neosauropod eusauropods. It remains possible that Janenschia retains plesiomorphic presacral vertebrae (i.e. Tendaguria is a junior synonym) and/or an unusual caudal morphology (i.e. Wamweracaudia is a junior synonym) (see also Britt et al., 2017). Similarly, it is possible that Tendaguria and Wamweracaudia represent one taxon, although the presacral vertebrae of Mamenchisaurus (the sister taxon of Wamweracaudia in our analysis) are very different to those of Tendaguria. Janensch considered all of these remains as pertaining to one taxon, although he did not provide explicit reasons for their referral. It seems likely that he noted anatomical features in elements, now lost/destroyed, that he considered linked these individuals from different localities (e.g. caudal vertebrae from quarries $P$ and G). However, even if Janensch did observe 'unique' features justifying referrals, we have to consider to what extent these features have now decayed into widely distributed synapomorphies through the process of character obsolescence (Wilson \& Upchurch, 2003). For example, the lost caudal vertebrae from Quarry P might have been strongly procoelous, suggesting a close affinity with those from Quarry $G$, but we now know that procoely is widely distributed across several eusauropod lineages. Therefore, without clearly stated and/or figured autapomorphies for us to examine, it is not possible to assign non-overlapping remains to Janenschia without the risk of creating a chimera. Furthermore, whereas there was a greater tendency to lump taxa in the past, based on overall similarities, our increased sampling of taxa and therefore morphology means that splitting is more often advocated in modern taxonomy. For example, whereas Janensch regarded the Tendaguru brachiosaurid to belong to the North American genus Brachiosaurus, it is now considered to be a distinct genus (i.e. Giraffatitan; Taylor, 2009).

To test the effect of alternative phylogenetic placements (i.e. possible synonymies), we ran several constraints, forcing different combinations of these three taxa together (Table 18). In each case, the seven most unstable taxa were excluded a priori. Using equal weights, all of these constraints resulted in much poorer overall resolution and a greater number of steps (2-10) than our original analysis (Table 18). However, there is essentially no difference in resolution or numbers of steps when extended implied weights are applied (Table 18). When Wamweracaudia is constrained as the sister taxon of Janenschia, this grouping occupies the original (i.e. unconstrained) position of Janenschia. In contrast, when Tendaguria is constrained as the sister taxon of Janenschia, this grouping is placed in the original position of Tendaguria (and Haestasaurus also moves to this part of the tree). The original placement of Wamweracaudia is retained when this taxon is constrained as the sister taxon of Tendaguria. Finally, when all three taxa are constrained together, this grouping is placed in the original position of Janenschia. As such, most alternative positions 
are only slightly suboptimal, but there is no clear 'answer' as to whether these taxa are synonymous with one another.

Augmenting our current Janenschia OTU with scores for the manus (MB.R.2093) has no impact on numbers of MPTs or tree topology, and only minimal increase in tree length (one step for equal weights, and one decimal place for extended implied weights). As such, there is a strong possibility that the manus does indeed belong to Janenschia, as proposed by Janensch (1922), but it cannot be unequivocally referred. In summary, pending the discovery of new, associated individuals preserving overlapping elements, it is impossible to fully determine whether the Tendaguru sauropods are oversplit, but based on current information there is evidence for at least seven distinct genera.

\section{EUSAUROPOD PHYLOGENETIC RELATIONSHIPS}

Below, we focus on additional results from our phylogenetic analyses that are different to previous studies. Although there are some differences in our titanosauriform topology from previous iterations of this matrix (primarily within lithostrotian titanosaurs) (see Figs 41, 42), we do not discuss this part of the tree further given that character revision for Titanosauriformes was not the focus of this study. Any unusual results in that part of the tree should thus be treated with caution.

As noted in the preceding section, the Middle/Late Jurassic Chinese sauropod Bellusaurus lies outside of Neosauropoda in both sets of analyses (see also Mo, 2013), whereas some previous studies have recovered it as a non-titanosauriform macronarian (Upchurch et al., 2004; Carballido et al., 2012). However, it remains possible that the juvenile nature of the Bellusaurus skeletal remains impacts upon this position, given that immature individuals (and adult taxa retaining paedomorphic traits) can appear in a more basal position in the tree than their adult counterparts (e.g. Tsuihiji et al., 2011; Campione et al., 2013; Carballido \& Sander, 2014). Haestasaurus and Tehuelchesaurus have also been regarded as non-titanosauriform macronarians by most authors (e.g. Carballido et al., 2011; Upchurch et al., 2015), but the former lies outside of Neosauropoda in both of our analyses, and the latter is a non-neosauropod eusauropod in our EWP analysis.

As noted above, Haplocanthosaurus has a long history of being recovered as either a non-neosauropod eusauropod, a 'basal' diplodocoid, or a non-titanosauriform macronarian. Here, the two species of Haplocanthosaurus are placed as the most 'basal' members of Diplodocoidea in both of our analyses. In our EWP analysis, the two Haplocanthosaurus species ( $H$. priscus and $H$. delfsi) are recovered as sister taxa for the first time, whereas they are paraphyletic in our EIW analysis. Amphicoelias is also recovered as a 'basal' diplodocoid, outside of Diplodocimorpha (i.e. Flagellicaudata + Rebbachisauridae), in both analyses. This position is consistent with the analyses of Rauhut et al. (2005) and Whitlock (2011a), whereas Amphicoelias was recovered as an apatosaurine diplodocid in Tschopp \& Mateus (2017).

Within Diplodocinae, we recover Leinkupal and Tornieria as sister taxa, with this Gondwanan clade more closely related to Diplodocus than to Dinheirosaurus + Supersaurus, contrasting with the topology presented in Tschopp \& Mateus (2017). Our Dicraeosauridae largely maintains the same topology as that presented by $\mathrm{Xu}$ et al. (2018), with the Late Jurassic taxa Brachytrachelopan and Dicraeosaurus forming a clade to the exclusion of Amargasaurus (although they form a polytomy in our EIW analysis), and Lingwulong and Suuwassea recovered as successively more distant outgroups to these 'advanced' dicraeosaurids. However, in our EWP analysis, we also recover Cetiosauriscus as the sister 
taxon to Lingwulong. In contrast, Cetiosauriscus is positioned as a non-neosauropod eusauropod in our EIW analysis, forming a clade with the late Middle Jurassic North African taxon Jobaria. Although Cetiosauriscus has occasionally been recovered as a diplodocoid before (e.g. Tschopp et al., 2015a), it has always been placed near the base of the clade. As such, both a dicraeosaurid placement and a sister taxon relationship with Jobaria are novel to our analysis.

In general, our two rebbachisaurid topologies are similar to those of recent analyses (e.g. Whitlock, 2011a; Carballido et al., 2012; Mannion et al., 2012; Fanti et al., 2015; Wilson \& Allain, 2015). Amazonsaurus is the most 'basal' member of Rebbachisauridae in both analyses, with Histriasaurus its sister taxon in our EWP analysis. Comahuesaurus is also placed outside of Khebbashia (Limaysaurinae + Nigersaurinae) in both analyses. A fully resolved Nigersaurinae recovers Demandasaurus and the Isle of Wight caudal vertebra as sister taxa, with Tataouinea, Rebbachisaurus, and Nigersaurus as successively more 'basal' nigersaurines. The recovery of Rebbachisaurus within Nigersaurinae prompted Wilson and Allain (2015) to propose that Rebbachisaurinae should replace Nigersaurinae (Whitlock, 2011a), following Article 36 of the International Code of Zoological Nomenclature, which states that: "coordinate nominal taxa of the family group have the same type genus". However, the position of Nigersaurus as the sister taxon of all other members of Nigersaurinae (see also Fanti et al., 2015) means that Nigersaurinae and Rebbachisaurinae do not necessarily have to have the same composition, i.e. Rebbachisaurinae does not have to include Nigersaurus. As Nigersaurinae is now commonly used, and the position of Rebbachisaurus could change in future analyses, we recommend the continued use of Nigersaurinae as all rebbachisaurids more closely related to Nigersaurus than to Limaysaurus (Whitlock, 2011a). Nigersaurinae appears to have been restricted to North Africa and Europe. The remaining rebbachisaurid taxa form a polytomy with Nigersaurinae in our EWP analysis that is resolved when Katepensaurus and Zapalasaurus are pruned a posteriori. Limaysaurinae is composed exclusively of Argentinean taxa (see also Fanti et al., 2015), comprising Cathartesaura, Limaysaurus, Nopscaspondylus, and the El Chocon rebbachisaurid in both sets of analyses. Katepensaurus and Rayososaurus are additional limaysaurines in our EWP analysis, but lie outside of Khebbashia in our EIW analysis (along with Histriasaurus). Zapalasaurus forms a polytomy with Limaysaurinae and Nigersaurinae in our EWP analysis, but is placed as one of the most 'basal' rebbachisaurids in our EIW analysis.

The Middle Jurassic Moroccan sauropod Atlasaurus is recovered as a macronarian in both of our analyses: it is the sister taxon of all other members of this clade in our EWP analysis, whereas it is a brachiosaurid in our EIW analysis. Whereas recent analyses have placed Atlasaurus outside of Neosauropoda (e.g. D'Emic et al., 2016; Mannion et al., 2017), it has been recovered as a non-titanosauriform macronarian previously (Upchurch et al., 2004), and was originally suggested to be a brachiosaurid by Monbaron et al. (1999). However, all of these placements should be treated with caution: until Atlasaurus is properly described, it will not be possible to determine its phylogenetic affinities with any confidence.

Lourinhasaurus is the sister taxon to Camarasaurus in both analyses, supporting most previous studies that have tended to find them as closely related (see Mocho et al., 2014). Whereas this clade (Camarasauridae) occupies its traditional non-titanosauriform macronarian placement in our EWP analysis, it is the sister taxon to Neosauropoda in our EIW analysis. Although some authors have proposed that Lourinhasaurus represents a nonneosauropod (e.g. Upchurch et al., 2004), Camarasaurus has been universally regarded as a member of Macronaria, making this result extremely unexpected. 
TURIASAURS OUTSIDE OF EURAMERICA?

Royo-Torres \& Cobos (2009) suggested that some of the remains referred to Janenschia might belong to Turiasauria, a clade of non-neosauropod eusauropods previously known only from the latest Jurassic-earliest Cretaceous of western Europe (Royo-Torres et al., 2006; Royo-Torres et al., 2009; Néraudeau et al., 2012; Royo-Torres \& Upchurch, 2012; Mateus et al., 2014; Mocho et al., 2016) and late Early Cretaceous of North America (RoyoTorres et al., 2017a). In particular, they discussed the caudal sequence (now the holotype of Wamweracaudia), the humerus and an astragalus from Quarry P (referable to Janenschia), and the manus from Nr. 5 (regarded here as Eusauropoda indet.). Most of the features proposed by Royo-Torres \& Cobos (2009) to support a turiasaurian identification are plesiomorphic for Sauropoda or a slightly more exclusive clade (i.e. caudal centra without lateral pneumatic foramina; strongly posteriorly projecting caudal neural spines; proximally bridged chevrons; anteriorly projecting humeral deltopectoral crest; short metacarpal I; long manual ungual on digit I), and thus cannot be used to assign these remains to Turiasauria.

Royo-Torres \& Cobos (2009) also commented upon the shared presence of procoelous anterior caudal centra, but the degree of convexity is much weaker in Losillasaurus (Casanovas et al., 2001) and remains tentatively referred to Turiasaurus (Royo-Torres et al., 2009) than in Wamweracaudia (see Table 10). On the other hand, the degree of convexity in the recently described turiasaur Moabosaurus is equivalent (Britt et al., 2017) but, regardless, the presence of procoelous anterior caudal centra is widespread among an array of eusauropod lineages (Bonaparte et al., 2000; Whitlock et al., 2011; Mannion et al., 2013; Mocho et al., 2017a).

The remaining feature used by Royo-Torres \& Cobos (2009) to unite Turiasaurus and Wamweracaudia pertains to the anterior orientation of the caudal ribs (note that Losillasaurus has posterolaterally curving caudal ribs [Casanovas et al., 2001; MCNV Lo: PDM \& PU pers. obs. 2009]). However, whereas the caudal ribs of Wamweracaudia curve strongly anterolaterally, those of Turiasaurus merely decrease in breadth towards their lateral tips along their posterior margin, with the anterior margin remaining straight in dorsal view (Royo-Torres et al., 2009: fig. 3q). Furthermore, three unrelated taxa (the diplodocids Leinkupal and Tornieria, and the titanosaur Xianshanosaurus) also have anteriorly deflected caudal ribs.

Although both sets of our analyses suggest that Wamweracaudia lies outside of Turiasauria, they indicate a novel position for Tendaguria as a turiasaur. Furthermore, our EWP analysis also recovers Janenschia within Turiasauria. As such, it seems that at least one turiasaur was present in the Late Jurassic Tendaguru Formation, indicating that the clade was not restricted to Euramerica. Some of our analyses also recover Tehuelchesaurus and Bellusaurus, from Argentina and China respectively, as turiasaurs. If correct, this would mean that Turiasauria was a near-global radiation (see 'Biogeographic origins of the Tendaguru sauropod fauna') below.

\section{MAMENCHISAURIDS OUTSIDE OF EAST ASIA?}

Mamenchisauridae is defined as the most inclusive clade that includes Mamenchisaurus constructus but excludes Saltasaurus loricatus (Naish \& Martill, 2007), and has generally been regarded as endemic to East Asia (Russell \& Zheng, 1993). The type species, Mamenchisaurus constructus, from the late Middle Jurassic of Sichuan (Xing et al., 2015), in southwest China, was named by Young (1954). Additional remains attributed to 
Mamenchisaurus, including multiple new species, were subsequently described from the Middle-Late Jurassic of Sichuan (e.g. Young \& Zhao, 1972; Ouyang \& Ye, 2002), with further occurrences also documented from contemporaneous outcrops in neighbouring Chongqing (Xing et al., 2015), northwest China (e.g. Young, 1958; Russell \& Zheng, 1993), and western Mongolia (Graham et al., 1997). Fragmentary remains have also been referred to Mamenchisaurus from the Tithonian/Berriasian of northeast Thailand (e.g. Suteethorn et al., 2013). A putative occurrence of Mamenchisaurus from the Early Cretaceous of Japan (Hasegawa et al., 1991) has since been regarded as an indeterminate sauropod (Azuma \& Tomida, 1998; Barrett et al., 2002). As well as numerous species of Mamenchisaurus (of which several might not belong to this genus [Sekiya, 2011; Xing et al., 2015]), a number of additional East Asian genera have been included within Mamenchisauridae. Of these, the mamenchisaurid affinities of Chuanjiesaurus (Sekiya, 2011; this study) and Qijianglong (Xing et al., 2015) seem most secure. Other putative East Asian Middle-Late Jurassic mamenchisaurids comprise Eomamenchisaurus (Lü et al., 2008), Huangshanlong (Huang et al., 2014), Klamelisaurus (Moore, Xu \& Clark, 2017; IVPP V9492: PU pers. obs. 2010), Omeisaurus (e.g. He et al., 1988; Wilson, 2002), Tienshanosaurus (Sekiya, 2011), Yuanmousaurus (Sekiya, 2011), and Xinjiangtitan (Wu et al., 2013), and are all restricted to western China. Tonganosaurus, from the Early Jurassic of Sichuan, has been suggested to represent an earlier mamenchisaurid occurrence (Li et al., 2010; Xing et al., 2015), but this placement was based on overall similarities with Omeisaurus (which itself has sometimes been recovered outside Mamenchisauridae [e.g. Upchurch et al., 2004; this study]), rather than through phylogenetic analysis.

If correct, the recovery of the newly erected southern Gondwanan Late Jurassic taxon Wamweracaudia within Mamenchisauridae indicates that this clade was not endemic to East Asia. Given that most evidence indicates that East Asia was separated from the rest of Pangaea during the Late Jurassic (see reviews in Russell, 1993; Upchurch, 1995; Barrett et al., 2002; Upchurch, Hunn \& Norman, 2002; Wilson \& Upchurch, 2009), the phylogenetic position of Wamweracaudia suggests an earlier and more widespread diversification of mamenchisaurids prior to this geographical isolation (see 'Biogeographic origins of the Tendaguru sauropod fauna' below). Evidence for additional non-East Asian mamenchisaurids comes from a phylogenetic analysis presented by Rauhut et al. (2005). These authors recovered the Tithonian/Berriasian Spanish genus Losillasaurus (Casanovas et al. 2001) and the late Middle Jurassic UK taxon Cetiosauriscus (Woodward, 1905) as mamenchisaurids. Remes et al. (2009) also commented upon similarities between the Middle Jurassic North African sauropod Spinophorosaurus and East Asian mamenchisaurids. Losillasaurus has since been recovered as a member of Turiasauria (Royo-Torres, Cobos \& Alcalá, 2006) in nearly all studies, and a non-mamenchisaurid, turiasaurian placement is supported in our analysis. Both our analyses and those of Tschopp et al. (2015a) recovered Cetiosauriscus as either a diplodocoid or non-neosauropod eusauropod, and in neither case did it cluster with mamenchisaurids. Lastly, mamenchisaurid affinities for Spinophorosaurus have yet to receive support when this taxon is included in phylogenetic analyses (Remes et al., 2009; Nair \& Salisbury, 2012). Consequently, Wamweracaudia is currently the only extra-Asian taxon whose placement within Mamenchisauridae is well supported.

\section{TENDAGURU SAUROPOD FAUNAL COMPOSITION AND GLOBAL COMPARISONS}

The Late Jurassic (Kimmeridgian-Tithonian) Tendaguru Formation has yielded evidence for at least seven sauropod genera, comprising one dicraeosaurid (Dicraeosaurus), a 
diplodocid (Tornieria), a brachiosaurid (Giraffatitan), a second titanosauriform (Australodocus), a turiasaur (Tendaguria), a mamenchisaurid (Wamweracaudia), and a second non-neosauropod eusauropod (Janenschia). Additional unnamed material might indicate higher diversity (Remes, 2009; see also the Nr. 5 manus). Several other geographical regions with Middle Jurassic-earliest Cretaceous deposits preserve diverse eusauropod faunas (Fig. 45), and brief comparisons are made below (see also Mateus, 2006; Whitlock, 2011b; Mocho et al., 2014).

\section{North America}

Whereas there is almost no terrestrial record for the Middle Jurassic of North America, the Late Jurassic (Oxfordian-Tithonian) Morrison Formation of the western USA has an extremely high diversity of sauropods. Most of these are diplodocids, comprising the apatosaurines Apatosaurus and Brontosaurus, as well as several diplodocine genera: Barosaurus, Diplodocus, Galeamopus, Kaatedocus, and Supersaurus (McIntosh, 1990, 2005; Upchurch et al., 2004; Lovelace et al., 2008; Whitlock, 2011a; Tschopp \& Mateus, 2013; Tschopp et al., 2015a). There is also the dicraeosaurid Suuwassea (Salgado, Carvalho \& Garrido, 2006; Whitlock, 2011a), the titanosauriform Brachiosaurus (Taylor, 2009), the nontitanosauriform macronarian Camarasaurus (Upchurch, 1998; Wilson \& Sereno, 1998), and the 'basal' diplodocoids Amphicoelias and Haplocanthosaurus (based on our analyses; see also Whitlock, 2011a).

The earliest Cretaceous North American sauropod record is limited to fragmentary remains of a forelimb, possibly representing a basal macronarian, from a late BerriasianValanginian section of the Lakota Formation, in the western USA (D'Emic \& Foster, 2016). The next stratigraphically youngest North American sauropods are from the lower sections of the Cedar Mountain Formation (Yellow Cat and Poison Strip Sandstone members) in the western USA, which are generally regarded as Barremian-Aptian, although there is some evidence to suggest that they might be earliest Cretaceous (Royo-Torres et al., 2017a). These units have yielded the brachiosaurids Cedarosaurus (Tidwell, Carpenter \& Brooks, 1999) and Venenosaurus (Tidwell, Carpenter \& Meyer, 2001), and the turiasaurs Mierasaurus (RoyoTorres et al., 2017a) and Moabosaurus (Britt et al., 2017). Stratigraphically younger deposits (Aptian-early Cenomanian) in the USA have yielded further brachiosaurids (including Abydosaurus [Chure et al., 2010] and Sonorasaurus [D'Emic, Foreman \& Jud, 2016]), as well as remains of numerous somphospondylans, including Astrophocaudia and Sauroposeidon (e.g. Wedel et al., 2000; Rose, 2007; D’Emic \& Foreman, 2012; D’Emic, 2013).

\section{Europe}

The best records of Middle Jurassic-earliest Cretaceous European sauropods are from the UK and Iberia. In addition to Cetiosauriscus, from the Callovian Oxford Clay Formation (Woodward, 1905), Middle Jurassic UK sauropods are represented by the non-neosauropod eusauropod Cetiosaurus, from several Bajocian-Bathonian localities (Upchurch \& Martin, 2002,2003 ), along with teeth that have been suggested to belong to turiasaurs (Royo-Torres \& Upchurch, 2012). Several Middle Jurassic remains from the UK have been suggested to represent diplodocoids or titanosauriforms, but these and additional specimens are too fragmentary to refer them to particular taxa, and can only be regarded as indeterminate eusauropods (e.g. Upchurch \& Martin, 2003; Naish \& Martill, 2007; Whitlock, 2011a; Mannion et al., 2012, 2013; Manning et al., 2015).

In the Late Jurassic, all UK sauropod remains are fragmentary and largely nondiagnostic beyond Eusauropoda, with the probable titanosauriform Duriatitan 
humerocristatus (Kimmeridgian) the only potentially valid taxon (Barrett, Benson \& Upchurch, 2010). Mocho et al. (2016) have also suggested that some Late Jurassic teeth from the UK might be referable to Turiasauria. In the earliest Cretaceous (BerriasianHauterivian) sections of the Wealden Supergroup, UK sauropods are represented by Haestasaurus (the sister taxon of Janenschia), several titanosauriforms (probably belonging to Somphospondyli, e.g. Pelorosaurus conybeari [Upchurch, Mannion \& Barrett, 2011a]), and Xenoposeidon (Taylor \& Naish, 2007). The affinities of the latter remain enigmatic, but it is possible that it represents a rebbachisaurid (Taylor, 2018). Stratigraphically younger deposits in the Wealden Supergroup (Barremian-early Aptian) demonstrate the unambiguous presence of rebbachisaurids (Mannion et al., 2011), as well as multiple somphospondylans (Upchurch et al., 2011a; Mannion et al., 2013), including the oldest known titanosaur (D’Emic, 2012).

In Portugal, the Late Jurassic (late Kimmeridgian-early Tithonian) Lourinhã Formation (and other contemporaneous Portuguese deposits) has produced at least one diplodocid (Dinheirosaurus [Bonaparte \& Mateus, 1999; Mannion et al., 2012]), a brachiosaurid (Lusotitan [Mannion et al., 2013; Mocho, Royo-Torres \& Ortega, 2017b]), a nontitanosauriform macronarian (Lourinhasaurus [Royo-Torres et al., 2006; Mocho et al., 2014]; though see Upchurch et al. [2004] and our EIW analysis for a non-neosauropod eusauropod identification), and a turiasaur (Zby [Mateus et al., 2014]). Also in Iberia, the Spanish Villar Del Arzobispo Formation (Tithonian/Berriasian) records the non-titanosauriform macronarian Galveosaurus (Barco, 2006; Carballido et al., 2011; Mannion et al., 2013, 2017; though see Royo-Torres et al. [2006] and Royo-Torres \& Upchurch [2012] for a turiasaurian placement), an unnamed diplodocid (Royo-Torres et al., 2009), 'basal' titanosauriform remains (Suñer, Santisteban \& Galobart, 2009; Royo-Torres et al., 2014), and the turiasaurs Losillasaurus and Turiasaurus (Royo-Torres et al., 2006). The stratigraphic position of the Spanish non-titanosauriform macronarian Aragosaurus (Sanz et al., 1987; Mannion et al., 2013; Royo-Torres et al., 2014) has been the subject of debate (Canudo et al., 2012; RoyoTorres et al., 2014), but it has most recently been placed in the lower section of the Galve Formation and assigned a probable late Berriasian-early Valanginian age (Aurell et al., 2016). Stratigraphically younger Spanish deposits (late Hauterivian-early Aptian) record the presence of brachiosaurid (Soriatitan) and somphospondylan (Europatitan, Tastavinsaurus) titanosauriforms (Canudo et al., 2008; Royo-Torres et al., 2017b; Torcida Fernández-Baldor et al., 2017), as well as the rebbachisaurid Demandasaurus (Pereda-Suberbiola et al., 2003; Torcida Fernández-Baldor et al., 2011).

Asia

Most of the Middle Jurassic-earliest Cretaceous Asian sauropod record is from East Asia, primarily China. The Chinese Shishigou Formation (Callovian-Oxfordian), Shaximiao (=Dashanpu) Formation (generally regarded as Bathonian-Callovian, but recently dated as Oxfordian by Wang et al. [2018]), and other contemporaneous Middle-Late Jurassic East Asian outcrops, are dominated by mamenchisaurids and other non-neosauropods (Russell \& Zheng, 1993; Upchurch et al., 2004; Wings, Schwarz-Wings \& Fowler, 2011; Xing et al., 2015). Although a number of authors have stated that no unambiguous neosauropods are present in the pre-Cretaceous of Asia (e.g. Wilson, 2005b; Mannion et al., 2013), several taxa have been suggested to represent macronarians. In addition to Bellusaurus (see above), these include Abrosaurus (Upchurch et al., 2004) and Daanosaurus (Ye, Gao \& Jiang, 2005; D'Emic, 2012). However, neither of these latter two taxa have been described in detail, or included in a phylogenetic analysis, and thus their neosauropod affinities should be treated with 
caution (Upchurch et al., 2004; Mannion et al., 2013). Ferganasaurus, from the Callovian Balabansai Formation of Kyrgyzstan, was also described as a neosauropod (Alifanov \& Averianov, 2003). However, this was based on Ferganasaurus being placed in a polytomy with diplodocoids, macronarians, and Jobaria (Alifanov \& Averianov, 2003), and a subsequent analysis recovered it as a non-neosauropod eusauropod (Läng \& Mahammed, 2010). Most recently, Xu et al. (2018) described the dicraeosaurid Lingwulong, from the latest Early or early Middle Jurassic of northern China, demonstrating that Neosauropoda was present in the Jurassic of Asia. Regardless, in terms of the dominance of nonneosauropods, the composition of East Asian Middle-Late Jurassic sauropod faunas is quite different to that of contemporaneous European and North and South American formations, but shows a potentially unique overlap with that of the Tendaguru Formation in terms of the shared presence of mamenchisaurids. In addition, the recovery here of a non-neosauropod clade including Janenschia and Bellusaurus (plus Tehuelchesaurus in our EIW analysis) also suggests a possible closer relationship between East Asia and southern Gondwana than previously known.

In the Early Cretaceous, Asian sauropods appear to be entirely represented by somphospondylans (Whitlock et al., 2011; D'Emic, 2012; Mannion et al., 2013; Averianov \& Sues, 2017). Although most of these remains come from deposits dated as Barremian or younger, the revised age (Berriasian-Valanginian) for Euhelopus (see above) indicates that Somphospondyli was present in Asia by the earliest Cretaceous. Some of these Early Cretaceous somphospondylans might represent titanosaurs, with the stratigraphically oldest candidate being Tengrisaurus starkovi from the Barremian-Aptian Murtoi Formation of eastern Russia (Averianov \& Skutschas, 2017).

\section{South America}

Middle Jurassic South American sauropods are restricted to a small number of localities in the upper sections of the Cañadón Asfalto Formation (Aalenian-Bajocian) of southern Argentina (Cúneo et al., 2013). These remains are primarily attributed to Patagosaurus (Bonaparte, 1986a; Rauhut, 2003), which is often recovered as the sister taxon to Cetiosaurus (e.g. Upchurch et al., 2004; Carballido et al., 2012). A second sauropod taxon, Volkheimeria (Bonaparte, 1986a), is also present in this formation, although its affinities remain uncertain (Upchurch et al., 2004). A greater diversity is indicated by teeth (Holwerda, Pol \& Rauhut, 2015), and Rauhut (2003) noted that some remains referred to Patagosaurus likely represent a distinct taxon. A further non-neosauropod eusauropod has been recovered from the lower section of the Cañadón Asfalto Formation, which is dated to the Toarcian (late Early Jurassic [Cúneo et al., 2013]), although it is yet to be described (Pol, Rauhut \& Carballido, 2009).

The Late Jurassic (Kimmeridgian-early Tithonian) Cañadón Calcáreo Formation of southern Argentina has so far yielded a dicraeosaurid (Brachytrachelopan [Rauhut et al., 2005]), a non-titanosauriform macronarian or non-neosauropod eusauropod (Tehuelchesaurus [Carballido et al., 2011]), and indeterminate remains belonging to a 'basal' titanosauriform (possibly a brachiosaurid; Rauhut, 2006; Mannion et al., 2013) and diplodocid (Rauhut, Carballido \& Pol, 2015). A locality in the Tithonian Toqui Formation of southern Chile also demonstrates the presence of a diplodocine in the Late Jurassic of South America (Salgado et al., 2015). A second diplodocine, Leinkupal, was described from the earliest Cretaceous (late Berriasian-Valanginian) Bajada Colorada Formation of southern Argentina, and indeterminate dicraeosaurid remains were also reported from this locality (Gallina et al., 2014). Indeterminate diplodocine and dicraeosaurid remains have also been 
documented from a nearby locality in the Valanginian Mulichinco Formation (Gnaedinger et al., 2017; Paulina Carabajal et al., 2018). These Late Jurassic-earliest Cretaceous South American outcrops are the closest palaeogeographically to the Tendaguru Formation, and are situated at approximately the same palaeolatitude; thus, we might expect the greatest similarity between these sauropod faunas. Indeed, Brachytrachelopan has been recovered as the sister taxon to Dicraeosaurus by most authors (e.g. Rauhut et al., 2005; Whitlock, 2011a; this study), and a close relationship between Janenschia and Tehuelchesaurus is recovered in our EWP analysis. Leinkupal is also recovered as the sister taxon to Tornieria in our analyses. Consequently, poor sampling of these South American formations might account for their lower diversity relative to that of the Tendaguru Formation, and new discoveries might fill the 'gaps' in their faunal lists.

Recently described remains (including Triunfosaurus leonardii) from the Rio Piranhas Formation (Hauterivian-Barremian) of northeastern Brazil have been assigned to Titanosauria (Ghilardi et al., 2016; Carvalho et al., 2017), although Poropat et al. (2017) argued that these could not be confidently referred beyond Somphospondyli. The discovery of Padillasaurus in the late Barremian Paja Formation of Colombia (Carballido et al., 2015) confirms the presence of Somphospondyli in the pre-Aptian of South America (Mannion et al., 2017). The Barremian-early Aptian La Amarga Formation of southern Argentina records the dicraeosaurids Amargasaurus (Salgado \& Bonaparte, 1991) and Amargatitanis (Gallina, 2016), the rebbachisaurid Zapalasaurus (Salgado et al., 2006), and remains of indeterminate titanosauriforms (Apesteguía, 2007).

\section{Africa}

The Middle Jurassic sauropod record of Africa is sparse, limited to three regions (Nicholl, Mannion \& Barrett, 2018). The Bathonian of Morocco has yielded the phylogenetically unstable (see above) eusauropod Atlasaurus (Guettioua Formation; Monbaron et al., 1999), as well as the non-neosauropod eusauropod 'Cetiosaurus' mogrebiensis (El Mers Formation; Lapparent, 1955; see also Upchurch \& Martin, 2003; Läng \& Mahammed, 2010). Another non-neosauropod eusauropod, Chebsaurus, is known from the Callovian Aïssa Formation of Algeria (Mahammed et al., 2005; Läng \& Mahammed, 2010). Two sauropods have been described from the Irhazer Group in Niger, comprising the non-eusauropod sauropod Spinophorosaurus (Bajocian-Bathonian; Remes et al., 2009), and the non-neosauropod eusauropod Jobaria (Bathonian-Callovian; Sereno et al., 1999; Rauhut \& López-Arbarello, 2009). Madagscar was still in contact with Africa until the Late Jurassic (Seton et al., 2012), and so is discussed here too. The Bathonian-aged Isalo III Formation of northwest Madagascar has yielded remains of several non-neosauropod eusauropods, comprising Lapparentosaurus, Archaeodontosaurus, and 'Bothriospondylus madagascariensis' (Bonaparte, 1986b; Buffetaut, 2005; Mannion, 2010).

Few African sauropod remains can be confidently dated to the Late Jurassic outside of the Tendaguru and Kadzi formations, and these are generally too incomplete to assign to particular clades. The earliest Cretaceous (Berriasian-Valanginian [or possibly latest Jurassic]) Kirkwood Formation of South Africa preserves fragmentary remains attributed to Diplodocidae, Dicraeosauridae, and Brachiosauridae, all of which appear closely related to taxa from the Tendaguru Formation (McPhee et al., 2016). An additional specimen from this stratigraphic unit was tentatively considered to represent either a non-titanosauriform macronarian or a non-neosauropod eusauropod (McPhee et al., 2016). Although recovered from present-day Croatia, the late Hauterivian-early Barremian deposits that yielded the 'basal' rebbachisaurid Histriasaurus were part of Afro-Arabia at the time (Dalla Vecchia, 
2005). The remainder of the pre-Aptian Cretaceous African sauropod record primarily comprises fragmentary and poorly dated remains from Saharan Africa (Lapparent, 1960). These indicate the presence of titanosauriforms and rebbachisaurids, but are in need of revision (Mannion \& Barrett, 2013).

Australia

The pre-Cretaceous sauropod record of Australia is restricted to Rhoetosaurus brownei, from the Bathonian-Callovian Walloon Coal Measures of Queensland (Nair \& Salisbury, 2012). Rhoetosaurus still awaits a detailed description, but Nair \& Salisbury (2012) recovered it as the sister taxon to Eusauropoda. The stratigraphically oldest Cretaceous Australian sauropod body fossils are from the late Albian, and appear to be entirely represented by somphospondylans (Poropat et al., 2016, 2017).

\section{Summary}

The Late Jurassic Tendaguru Formation shares representatives of the majority of eusauropod lineages with late Middle Jurassic to earliest Cretaceous global faunas. However, it displays a greater range of diversity than each of the other formations/regions by themselves. The exception is the lack of Rebbachisauridae, and clear evidence for basal neosauropods (either non-titanosauriform macronarians or non-diplodocimorph diplodocoids) in the Tendaguru Formation. However, the pre-Barremian fossil evidence for Rebbachisauridae is tentative, based solely on a fragmentary vertebra (Taylor, 2018), and it should be noted that the absent basal neosauropods represent paraphyletic grades, rather than distinct lineages.

\section{BIOGEOGRAPHIC ORIGINS OF THE TENDAGURU SAUROPOD FAUNA}

The results of our four BioGeoBEARS analyses provide an opportunity to assess the events which led to the assembly of the Tendaguru fauna. Before examining this issue, however, it is important to acknowledge some caveats and limitations on our interpretations. In particular, despite our modification to the geographic ranges that take into account some aspects of uneven spatiotemporal sampling, it remains the case that the latter is likely to have distorted the true biogeographic history of sauropods. For example, although we present the largest phylogenetic trees for sauropods thus far, our agreement subtrees include only 95 (EWP) or 99 (EIW) taxa, which is still less than $50 \%$ of currently known valid sauropodomorph species (Upchurch et al., 2011b). Moreover, it is clear from comparisons of the results of the harsh and relaxed analyses of the EWP and EIW trees that differences in both palaeogeographic constraints and in phylogenetic topology result in different ancestral area estimations (see below). As with all similar macroevolutionary analyses, the hypotheses presented below will need to be tested further through the discovery of more taxa, integration of existing taxa into larger phylogenies, refinement of palaeogeographic constraints, improvements in methodologies (especially with regard to the treatment of the sampling issue), and more precise dating of true (rather than minimum) divergence times.

The agreement subtrees include six of the seven Tendaguru taxa: (1) the dicraeosaurid Dicraeosaurus; (2) the brachiosaurid Giraffatitan; (3) the non-neosauropod eusauropod Janenschia; (4) the turiasaur Tendaguria; (5) the diplodocid Tornieria; and (6) the mamenchisaurid Wamweracaudia. The biogeographic origin of each of these taxa is discussed below. 
Dicraeosaurus is the sister taxon of Brachytrachelopan in the EWP subtree, but of Amargasaurus in the EIW tree. This difference, however, reflects the pruning of unstable taxa, with Brachytrachelopan being absent from the EIW subtree because of its instability. In either case, however, Dicraeosaurus has a South American sister taxon. In both the harsh and relaxed EWP analyses, the most recent common ancestor (MRCA) of Dicraeosaurus and Brachytrachelopan is present in South America, and the lineage leading to the former then dispersed into Africa during, or prior to, the Kimmeridgian. The harsh and relaxed EIW analyses produce a similar result, except that the MRCA of Dicraeosaurus and Amargasaurus occurs in Asia and South America prior to the dispersal into Africa. These results indicate that tree topology (and perhaps especially the pruning of Brachytrachelopan) has affected the ancestral area estimations, whereas the alternative palaeogeographic constraints make no difference. At no point during the Mesozoic did Asia and South America form a coherent geographic unit to the exclusion of other regions (e.g. see Xu et al. [2018] and references therein). Thus, the EIW results potentially reflect the impact of sampling biases, with the Asia-South America ancestral area estimation instead indicating the presence of a more widespread distribution that would have also involved intervening areas such as Europe and Africa. This is consistent with the evidence that dicraeosaurids formed a widespread clade across much of Pangaea during the Middle and Late Jurassic, with Suuwassea in North America, Lingwulong in Asia, Dicraeosaurus in Africa, Brachytrachelopan in South America, and possibly Cetiosauriscus in Europe (the latter is only supported by the EWP results). In short, the presence of Dicraeosaurus in the Tendaguru fauna is currently best explained as the result of a dispersal event (most probably from South America), as part of the near global expansion of Dicraeosauridae during the Middle and Late Jurassic. According to our results, the underlying biogeographic process responsible for the presence of Dicraeosaurus was dispersal followed by founder-event speciation.

Giraffatitan is the sister taxon of Sonorasaurus in the EWP tree, and Brachiosaurus in the EIW tree: thus, it has a North American sister taxon in both topologies. Unlike Dicraeosaurus, the relationships of Giraffatitan are genuinely different in the two phylogenetic analyses, rather than being the result of a posteriori taxon pruning. Here, the choice of palaeogeographic constraint affects the ancestral area estimations more than tree topology. In the EWP and EIW harsh analyses, the MRCA of Giraffatitan and its sister taxon is depicted as occurring in North America and Europe, and then dispersing into Africa during or before the Kimmeridgian to give rise to Giraffatitan. In contrast, the EWP and EIW relaxed analyses estimate that the MRCA was present in Africa, and that dispersal into North America during or before the Kimmeridgian gave rise to Brachiosaurus, or dispersal during the Late Jurassic or Early Cretaceous gave rise to Sonorasaurus. Interestingly, both scenarios suggest faunal exchange between Laurasia and Gondwana during the Late Jurassic, a result that is consistent with older hypotheses concerning the Tendaguru and Morrison formations that were developed when Giraffatitan and Brachiosaurus were regarded as congeneric (e.g. Galton, 1977; Sereno et al., 1994). However, any land connection between North America and Africa (which would have to have been via South America after the Early Jurassic) was probably severed in the late Middle Jurassic (Smith, Smith \& Funnell, 1994; see also the review of palaeogeographic events during the Mesozoic in Poropat et al. [2016]), and it seems probable that any Europe-Africa landbridge did not form until the late Early Cretaceous (e.g. Dal Sasso et al., 2016; see below). The absence of evidence for a Late Jurassic land route between North America and Africa (usually via Europe) (e.g. Seton et al., 2012), the results of cladistic biogeographic analyses (e.g. Upchurch et al., 2002), and the decreased faunal similarity between Tendaguru and the Morrison formations resulting from 
taxonomic revisions (e.g. the restriction of Brachiosaurus to the North American material and its generic separation from Giraffatitan [Taylor, 2009]), have all combined to lead subsequent authors to cast doubt on faunal exchange between North America and Africa during the Late Jurassic (Upchurch et al., 2002). Moreover, it should be remembered that the timing of this putative North America-Africa faunal exchange can only be constrained as occurring during or before the Kimmeridgian. Thus, it is conceivable that the presence of these brachiosaurids in Africa and North America during the Late Jurassic is the result of a widespread distribution that occurred during the Middle Jurassic when Laurasia and Gondwana were still in contact (e.g. see Upchurch et al., 2002). Given that the earliest known unambiguous brachiosaurid, Vouivria, is found in the Oxfordian (early Late Jurassic) of Europe (Mannion et al., 2017), and the possibility that the late Middle Jurassic north African sauropod Atlasaurus might be a brachiosaurid (as suggested by our EIW analysis), the latter biogeographic history is plausible. The higher diversity of brachiosaurids in North America and Europe, the European forms often being placed in the 'basal' part of the brachiosaurid tree, and the observation that the earliest known unequivocal brachiosaurid material comes from Europe, all combine to suggest that this clade originated in Euramerica during the Middle Jurassic and then subsequently dispersed into Africa to give rise to Giraffatitan, as seen in the EWP and EIW harsh results (though see the above caveat regarding the possible brachiosaurid affinities of Atlasaurus, which could imply a northern Gondwanan origin instead). This scenario predicts that brachiosaurids should also be present in the Middle and/or Late Jurassic of South America and perhaps also Asia. Despite claims that South American brachiosaurids are known (i.e. Rauhut, 2006; Carballido et al., 2015), this view has not been supported by recent phylogenetic analyses and taxonomic revisions focussed on titanosauriforms: (1) specimens from the Kimmeridgian-early Tithonian Cañadón Calcáreo Formation of Argentina might ultimately be shown to represent a brachiosaurid (Rauhut, 2003), but lack clear synapomorphies of that clade (Mannion et al., 2013); and (2) Padilasaurus, from the late Barremian Paja Formation of Colombia, was originally identified as a brachiosaurid (Carballido et al., 2015), but appears to be represent a somphospondylan instead (Mannion et al., 2017). Although several sauropod specimens from Asia have been suggested to represent brachiosaurids (e.g. You \& Li, 2009), these have all been refuted (Ksepka \& Norell, 2010; Mannion, 2011; Mannion et al., 2013). Isolation of Asia potentially prevented brachiosaurids from invading this region, explaining their absence (e.g. see Ksepka \& Norell, 2010), but a sampling-based explanation cannot be ruled out (e.g. Xu et al., 2018). In short, the history leading to the presence of Giraffatitan in the Tendaguru fauna is uncertain at present, but we hypothesise a Middle Jurassic Brachiosauridae that originated in Euramerica and then dispersed to Africa. This might then have been followed by further dispersals of closer relatives of Giraffatitan back into North America (at least until the proto-North Atlantic Ocean precluded dispersal between Europe and North America possibly from the Barremian [Seton et al., 2012]), if the EPW and EIW relaxed results are correct. Such a dispersal northward from Africa is perhaps most likely to have occurred during the Early Cretaceous, when there is some evidence for Africa-Europe dispersals among other taxa (e.g. Gheerbrant \& Rage, 2006; Ezcurra \& Agnolin, 2012), and palaeogeographic support for a terrestrial connection between these regions via a series of emergent carbonate platforms during the Barremian-Albian (and possibly earlier) (e.g. Dalla Vecchia, 2005; Canudo et al., 2009; Dal Sasso et al., 2016).

Janenschia is the sister taxon of the earliest Cretaceous European Haestasaurus, and this pair are placed in a clade along with the Middle/Late Jurassic Chinese sauropod Bellusaurus as non-neosauropod eusauropods in both the EWP and EIW trees. However, the 
wider relationships of this clade differ in the two analyses. In the EWP tree, this clade is placed within Turiasauria, whereas in the EIW tree this clade is more closely related to Neosauropoda than is Turiasauria. The EWP harsh and relaxed analyses estimate that the MRCA of Janenschia and Haestasaurus occurred in Africa, and then dispersed into Europe during or before the Valanginian, resulting in the presence of the latter genus. The EIW harsh and relaxed analyses also agree that the MRCA was present in Africa, but suggest that this ancestor was more widespread, also occupying Asia. Although the Haestasaurus lineage appears to have dispersed from Africa to Europe during the Late Jurassic or Early Cretaceous, such an inference is problematic for several reasons. As noted above, there is no clear palaeogeographic mechanism available for such a direct dispersal. Laurasia and Gondwana probably lost contact during the late Middle Jurassic, and a Europe-Africa land connection is unlikely to have formed until the Barremian-Albian (Dal Sasso et al., 2016), postdating the Berriasian-Valanginian-aged Haestasaurus. One possibility is that the Haestasaurus lineage dispersed from Asia into Europe in the Early Cretaceous, given that Europe-Asia separation by the Russian Platform Sea was briefly interrupted in the early Berriasian (Baraboshkin et al., 2003; see Poropat et al., 2016; Xu et al., 2018). However, this dispersal event is supported (and that equivocally) by only one of our four analyses (EIW relaxed). Alternatively, given that the clade containing Bellusaurus, Janenschia, and Haestasaurus was potentially more widespread during the Middle Jurassic, it is possible that the latter genus represents a lineage descended from European members of that clade that diverged from African and Asian forms prior to the Late Jurassic. Thus, the apparent incongruence between the timing of the 'dispersal event' from Africa to Europe could be caused by missing data that obscures the true older divergence time for these lineages. In short, these results generally support the view that a clade of non-neosauropod eusauropods (perhaps also including Tehuelchesaurus) became widespread across a substantial portion of Pangaea (i.e. South America, Asia, Africa, and probably Europe) during the Middle Jurassic. Janenschia therefore potentially represents a relict species left over from this radiation.

Tendaguria is the sister taxon of the North American Moabosaurus, within Turiasauria, in both the EWP and EIW trees. The ancestral area estimations for the MRCA of Tendaguria and Moabosaurus are affected by both tree topology and palaeogeographic constraints, resulting in several competing biogeographic histories. The EPW harsh analysis suggests that the MRCA was widespread across North America and Africa during, or before, the Kimmeridgian, with subsequent regional extinctions reducing the range of the lineages leading to the two terminal taxa. The EPW relaxed analysis places the MRCA in North and South America, followed by a dispersal into Africa during or before the Kimmeridgian, to account for the presence of Tendaguria in the latter region. Finally, the EIW harsh and relaxed analyses estimate that the MRCA was widespread across Europe and North America, followed by dispersal into Africa during or before the Kimmeridgian: this latter scenario is essentially identical to that proposed for Giraffatitan by both the EWP and EIW harsh analyses. These results generally confirm the presence of turiasaurs in North America, Europe, and Africa (e.g. Royo-Torres et al., 2006, 2017a; Mocho et al., 2016). Although the EPW relaxed analysis also suggests that this clade occurred in South America (which is not unreasonable given their presence in Africa), there is currently no fossil evidence to confirm this. Direct dispersal between Euramerica and Africa during the Late Jurassic is unlikely palaeogeographically (see above), so we hypothesise that turiasaurs were probably widespread across at least these three areas, and perhaps also South America, during the Middle Jurassic. This inference receives some support from the proposal that 'heart'-shaped teeth from the Middle Jurassic of Europe belong to turiasaurs (Royo-Torres \& Upchurch, 
2012; Mocho et al., 2016). In short, the occurrence of Tendaguria in the Tendaguru fauna is likely to be the result of range expansion during the Middle Jurassic, followed by founderevent speciation in Africa at that time or later.

The diplodocines Tornieria from Tendaguru, and Leinkupal from South America, are sister taxa in both the EWP and EIW trees. The EWP harsh analysis suggests that the MRCA of these two taxa was present in South America. This was followed by dispersal into Africa (during or before the Kimmeridgian), and founder-event speciation in order to produce the lineage leading to Tornieria. However, the other three analyses all agree that this MRCA was present in Africa and that dispersal into South America gave rise to the lineage leading to Leinkupal. Lower nodes in the diplodocid trees are often estimated to have had ancestral ranges encompassing North and South America, and sometimes Asia. This suggests that diplodocids (which must have diverged from dicraeosaurids by the Bajocian or earlier, given the age of Lingwulong), achieved a widespread distribution across much of Pangaea during the Middle Jurassic. Given the inclusion of South America (and absence of Africa) in the ranges estimated for the lower diplodocid nodes, combined with the probability that the final land connection between Laurasia and Gondwana in the Jurassic was via North and South America in the Callovian (Smith et al., 1994; Ford \& Golonka, 2003; Iturralde-Vinent, 2006; Pindell \& Kennan, 2009; Seton et al., 2012), Leinkupal and Tornieria potentially represent the descendants of the southern hemisphere component of this Middle Jurassic radiation.

Wamweracaudia is a non-neosauropod eusauropod and the sister taxon of Mamenchisaurus in both the EWP and EIW trees. The EWP harsh and EIW relaxed analyses estimate that the MRCA of Wamweracaudia and Mamenchisaurus was present in Asia and Africa, and this is generally also the case for lower nodes within Mamenchisauridae. The EWP relaxed and EIW harsh results are similar to those just described, except that South America is substituted for Africa. These ancestral area estimations suggest that the mamenchisaurid clade had become widespread across Asia, Africa, and/or South America during the Middle Jurassic (i.e. Bathonian or earlier). This also implies the presence of mamenchisaurids in at least some of the intervening areas, such as Europe and North America at this time. If Wamweracaudia is truly a mamenchisaurid, and our biogeographic hypothesis is correct, the absence of direct fossil evidence would have to be explained as severe sampling failures during the Middle Jurassic, an interpretation supported by other studies of dinosaur diversity and fossil record quality such as Starrfelt and Liow (2016). Moreover, these results seriously undermine the view that mamenchisaurids represent an endemic East Asian radiation of non-neosauropod eusauropods produced by geographic isolation during the Middle and/or Late Jurassic (e.g. see Russell, 1993; Upchurch, 1995; Barrett et al., 2002; Wilson \& Upchurch, 2009; Xing et al., 2015), and thus reinforces the biogeographic conclusions of $\mathrm{Xu}$ et al. (2018). Our ancestral area estimations provide no clear indication of the probable centre of origin of mamenchisaurids, or of the directions and timings of their subsequent dispersals. Potentially, however, the presence of Wamweracaudia in the Tendaguru fauna reflects the persistence of a lineage in Africa since the Middle Jurassic, or a dispersal from South America into Africa at that time or during the early Late Jurassic. Interestingly, Wamweracaudia is not the only Tendaguru dinosaur that is the sister taxon of an East Asian form: the ceratosaurian theropod Elaphrosaurus from Tendaguru clusters with the Oxfordian Limusaurus from China in several recent phylogenies (e.g. Xu et al., 2009; Tortosa et al. 2014; Rauhut \& Carrano, 2016). In the absence of a quantitative biogeographic analysis for ceratosaurs, it would be premature to suggest that the Elaphrosaurus+Limusaurus lineage had a biogeographic history similar to that for 
mamenchisaurids. Nevertheless, this pairing of Late Jurassic ceratosaurs lends additional credence to the idea that the isolation of East Asia, and associated endemism, might not have been as profound as previously supposed (see Xu et al. [2018] for further discussion of this issue).

The results outlined above suggest that the Tendaguru sauropod fauna was assembled through a diverse and complex series of biogeographic events. At least some of this complexity is probably artefactual, resulting from phylogenetic errors and sampling failures. Nevertheless, several broad patterns can be discerned, and there are also some wider implications for our understanding of Jurassic terrestrial biogeography. Several nonneosauropod and neosauropod lineages had acquired very widespread, or perhaps global, distributions by the early Middle Jurassic. This is consistent with the view that sauropods, as large-bodied terrestrial animals, were unlikely to cross oceanic barriers, and so were constrained to disperse across Pangaea prior to the severing of land routes between Laurasia and Gondwana during the late Middle Jurassic. Within this broad over-arching pattern of multiple clades becoming widely distributed during the Middle Jurassic, three different biogeographic histories potentially explain the composition of the Tendaguru sauropod fauna. First, brachiosaurids and turiasaurs represent Euramerican radiations that dispersed into Gondwana during the Middle and/or early Late Jurassic. These groups then both suffered regional extinctions in the Early Cretaceous, resulting in their persistence solely in Euramerica (e.g. Chure et al., 2010; Royo-Torres et al., 2017a,b). Second, although the Diplodocoidea as a whole was a nearly global radiation that must have dispersed across much of Pangaea during the late Early or early Middle Jurassic (Xu et al., 2018), there is a distinct SamAfrica (=west Gondwanan) aspect to its representatives in the Tendaguru fauna. In particular, Dicraeosaurus clusters with the South American Amargasaurus and Brachytrachelopan, and Tornieria is the sister taxon of the South American Leinkupal. Moreover, although currently unknown from the Jurassic, the majority of rebbachisaurids are also restricted to South America and Africa during the Early Cretaceous and earliest Late Cretaceous, with the few forms reaching Europe from Africa via dispersal across a landbridge during the Barremian or later (e.g. Carballido et al., 2012; Fanti et al., 2015; though this scenario would require some revision if the late Berriasian-early Valanginian UK sauropod Xenoposeidon is a rebbachisaurid - see Taylor, 2018). This suggests that several separate diplodocoid lineages reached west Gondwana during the Middle Jurassic, and were then isolated from their northern relatives during the Late Jurassic and earliest Cretaceous, resulting in a series of endemic southern hemisphere radiations. Third, and finally, the two non-neosauropod eusauropod lineages comprising the mamenchisaurids and the Bellusaurus+Janenschia+Haestasaurus clade, potentially also became widespread across Pangaea during the Middle Jurassic, with their representatives in Tendaguru being 'relicts' that persisted after their clades underwent regional extinction and substantial range contractions in the Late Jurassic. The apparent phylogenetic partitioning of these biogeographic patterns, with brachiosaurids and turiasaurs as 'northern invaders', dicraeosaurids and Tornieria+Leinkupal as 'SamAfrican endemics', and the two other nonneosauropod lineages as 'relicts' of wider Middle Jurassic distributions, hints at the possible effects of differences in dispersal ability, and/or ecological requirements. These different histories might also reflect the impact of where and when each clade originated and its subsequent opportunities to disperse into other regions. However, at present, the nature of these differences remain obscure.

As well as undermining support for the East Asian Isolation hypothesis, and emphasising the poverty of the Middle Jurassic sauropod fossil record, our results have one 
additional wider implication for our understanding of Jurassic terrestrial biogeography. Recently, there has been increasing interest in the impact of climatic zonation on dinosaur distributions (e.g. Whiteside et al., 2011; Benson et al., 2012). In particular, a large arid area (the Central Gondwanan Desert [CGD]) might have hampered dispersal between northern and southern Gondwana in the Middle and Late Jurassic (Volkheimer 1969; Parrish 1993; Scotese, Boucot \& McKerrow, 1999; Rauhut \& Lopez-Arbarello, 2008, 2009; Remes et al., 2009; Pol and Rauhut 2012; Souto \& Fernandes, 2017). The CGD stretched across Africa and South America between $15^{\circ}$ and $30^{\circ} \mathrm{S}$ during the Late Jurassic and Early Cretaceous at least (Souto \& Fernandes, 2017). It probably formed during the Early Jurassic (Rauhut \& LopezArbarello, 2009; Remes et al., 2009), and persisted through to the Early Cretaceous (Souto \& Fernandes, 2017). Remes et al. (2009) proposed that the CGD divided early sauropod faunas into two, producing closer phylogenetic relationships among north African, European, and Asian forms to the north, and a distinct monophyletic lineage in Patagonia (see also Rauhut \& Lopez-Arbarello, 2009). Other studies have suggested that the CGD created a 'filter barrier', allowing some clades to develop global distributions, while others were restricted to southern Gondwana (Pol \& Rauhut, 2012). Dinosaur groups that apparently display a mixture of virtually globally distributed clades and others limited to southern Gondwana during the Jurassic include: tetanuran theropods (with 'basal' forms south of the CGD, and derived megalosauroids widespread across north Africa and Europe [Rauhut \& LopezArbarello, 2009); 'basal' eusauropods (Remes et al., 2009); and heterodontosaurid ornithischians (Pol, Rauhut \& Becerra, 2011) (see also Apesteguía, Gómez \& Rougier [2012] for an example from the Rhynchocephalia). One problem with this view, however, is that the absence of members of the southern Gondwanan groups in regions north of the CGD (and vice versa) might merely result from sampling failure. Indeed, it transpires that this is the case for ceratosaurs, which were initially believed to be present in the Middle Jurassic of north Africa and Asia, and absent in southern Gondwana (Rauhut \& Lopez-Arbarello, 2009), but have recently been recovered from the Aalenian of the latter area (Pol \& Rauhut, 2012).

Our biogeographic results suggest that several of the Tendaguru sauropods were derived from clades that probably originated in Laurasia (brachiosaurids, turiasaurs, mamenchisaurids), and/or became widespread across much of Pangaea (diplodocoids, Janenschia and its relatives) during the Middle Jurassic. Minimum divergence time estimates, coupled with the probable separation of Laurasia from Gondwana from the Callovian onwards, suggest that these sauropod lineages must have reached eastern Africa prior to the late Middle Jurassic. Given that Tendaguru was located south of the CGD, this would mean that multiple sauropod lineages would have crossed this apparent barrier during the Middle Jurassic. This is more consistent with the 'filter barrier' interpretation than with a stringent barrier that resulted in vicariance north and south of the CGD. A similar history of trans-CGD dispersals has also been postulated for amphilestherian mammals (Gaetano \& Rougier, 2012). The particular location of Tendaguru, on the east coast of Gondwana, might also have facilitated dispersals between northern and southern Gondwana. Fluctuations in climate potentially facilitated such dispersals, especially along the more humid eastern margin of the CGD (Sellwood \& Valdes, 2008; Volkheimer et al., 2008; Rauhut \& Lopez-Arbarello, 2009). In particular, the CGD seems to have contracted during the late Middle Jurassic (Remes et al., 2009), which is congruent with the requirement that sauropod lineages must have dispersed into or out of the Tendaguru region prior to the Callovian. If the CGD was not a major obstacle to the dispersal of sauropods across Gondwana, then the absence of neosauropod lineages, turiasaurs, and mamenchisaurids in the Middle Jurassic of Patagonia is even more anomalous. It is possible that local or regional 
differences in habitat led to the development of a sauropod fauna dissimilar to those prevailing elsewhere in Pangaea during this interval. Alternatively, and perhaps more plausibly given the scarcity of Middle and Late Jurassic sauropod remains from South America (see above), it might simply be that this reflects the random effects of random sampling failures.

\section{CONCLUSIONS}

Revision of a number of enigmatic sauropod dinosaur taxa and remains from the Late Jurassic Tendaguru Formation of Tanzania clarifies their taxonomy and phylogenetic affinities. We demonstrate the validity and non-neosauropod eusauropod placement of Janenschia robusta, and show its close relationship with the earliest Cretaceous UK sauropod Haestasaurus. Tendaguria tanzaniensis is recovered as a turiasaur, making it the first known Gondwanan representative of this clade. We erect a new taxon for the caudal vertebral sequence MB.R.2091.1-30, Wamweracaudia keranjei gen. et sp. nov, which represents a non-Asian representative of Mamenchisauridae. Australodocus most likely represents a nontitanosaurian somphospondylan, and is the only pre-Cretaceous occurrence of this clade known at present. The Tendaguru Formation shares representatives of nearly all sauropod lineages with contemporaneous global faunas, and indicates that some clades thought to be endemic to East Asia must have diversified prior to its Late Jurassic isolation from the rest of Pangaea. The results of our biogeographic analyses indicate that the Tendaguru fauna has complex origins, with each of its constituent taxa having a different biogeographic history. Nevertheless, it is clear that many non-neosauropod and neosauropod lineages radiated across much of Pangaea during the late Early and Middle Jurassic, resulting in their presence at Tendaguru. Moreover, three broad and phylogenetically distinct scenarios for the origins of the Tendaguru sauropod fauna can be identified: (1) clades such as brachiosaurids and turiasaurs that potentially originated in Euramerica and then dispersed into Africa; (2) nearglobally distributed diplodocoids that underwent an endemic radiation in SamAfrica; and (3) widespread non-neosauropod eusauropod lineages that underwent regional extinction in the Late Jurassic and left 'relicts' in Africa. Minimum divergence times and ancestral range estimations demonstrate that multiple sauropod lineages must have dispersed across the Central Gondwanan Desert during the Middle Jurassic, suggesting that this geographic feature was probably not responsible for vicariance between Laurasia+northern Gondwana and southern Gondwanan.

\section{REFERENCES}

Aberhan M, Bussert R, Heinrich W-D, Schrank E, Schultka S, Sames B, Kriwet J, Kapilima S. 2002. Palaeoecology and depositional environments of the Tendaguru Beds (Late Jurassic to Early Cretaceous, Tanzania). Mitteilungen aus dem Museum für Naturkunde in Berlin, Geowissenschaftliche Reihe 5: 17-42.

Alifanov VR, Averianov AO. 2003. Ferganasaurus verzilini, gen. et sp. nov., a new neosauropod (Dinosauria, Saurischia, Sauropoda) from the Middle Jurassic of Fergana Valley, Kirghizia. Journal of Vertebrate Paleontology 23: 358-372.

Apesteguía S. 2005a. Evolution of the titanosaur metacarpus. In: Tidwell V, Carpenter K, eds. Thunder-lizards: the Sauropodomorph Dinosaurs. Bloomington; Indianapolis: Indiana University Press, 321-345. 
Apesteguía S. 2005b. Evolution of the hyposphene-hypantrum complex within Sauropoda. In: Tidwell V, Carpenter K, eds. Thunder-lizards: the Sauropodomorph Dinosaurs. Bloomington, Indianapolis: Indiana University Press, 248-267.

Apesteguía S. 2007. The sauropod diversity of the La Amarga Formation (Barremian), Neuquén, (Argentina). Gondwana Research 12: 533-546.

Apesteguía S, Gallina PA, Haluza A. 2010. Not just a pretty face: anatomical peculiarities in the postcranium of rebbachisaurids (Sauropoda: Diplodocoidea). Historical Biology 22: 165-174.

Apesteguía S, Gómez RO, Rougier GW. 2012. A basal sphenodontian (Lepidosauria) from the Jurassic of Patagonia: new insights on the phylogeny and biogeography of Gondwanan rhynchocephalians. Zoological Journal of the Linnean Society 166: 342-360.

Arratia G, Kriwet J, Heinrich W-D. 2002. Selachians and actinopterygians from the Upper Jurassic of Tendaguru, Tanzania. Mitteilungen aus dem Museum für Naturkunde in Berlin, Geowissenschaftliche Reihe 5: 205-228.

Aurell M, Bádenas B, Gasca JM, Canudo JI, Liesa CL, Soria AR, Moreno-Azanza M, Najes L. 2016. Stratigraphy and evolution of the Galve sub-basin (Spain) in the middle Tithonianearly Barremian: Implications for the setting and age of some dinosaur fossil sites. Cretaceous Research 65: 138-162.

Averianov A, Skutschas P. 2017. A new lithostrotian titanosaur (Dinosauria, Sauropoda) from the Early Cretaceous of Transbaikalia, Russia. Biological Communications 62: 6-18.

Averianov A, Sues H-D. 2017. Review of Cretaceous sauropod dinosaurs from Central Asia. Cretaceous Research 69: 184-197.

Azuma Y, Tomida Y. 1998. Japanese dinosaurs. In: Curie PJ, Padian K, eds. Encyclopaedia of Dinosaurs. Academic Press, San Diego, 375-379.

Bandyopadhyay S, Gillette DD, Ray S, Sengupta DP. 2010. Osteology of Barapasaurus tagorei (Dinosauria: Sauropoda) from the Early Jurassic of India. Palaeontology 53: 533569.

Baraboshkin EY, Alekseev AS, Kopaevich LF. 2003. Cretaceous palaeogeography of the North-Eastern Peri-Tethys. Palaeogeography, Palaeoclimatology, Palaeoecology 196: 177208.

Barco JL, Canudo JI, Cuenca-Bescós G. 2006. Descripción de las vértebras cervicales de Galvesaurus herreroi Barco, Canudo, Cuenca-Bescós \& Ruiz-Omeñaca, 2005 (Dinosauria, Sauropoda) del tránsito Jurásico-Cretácico en Galve (Teruel, España). Revista Española de Paleontología 21: 189-205.

Barrett PM, Benson RBJ, Upchurch P. 2010. Dinosaurs of Dorset: part II, the sauropod dinosaurs (Saurischia, Sauropoda) with additional comments on the theropods. Proceedings of the Dorset Natural History and Archaeological Society 131: 113-126.

Barrett PM, Hasegawa Y, Manabe M, Isaji S, Matsuoka H. 2002. Sauropod dinosaurs from the Lower Cretaceous of eastern Asia: taxonomic and biogeographical implications. Palaeontology 45: 1197-1217.

Bell MA, Lloyd GT. 2015. strap: an R package for plotting phylogenies against stratigraphy and assessing their stratigraphic congruence. Palaeontology 58: 379-389.

Benson RBJ, Rich TH, Vickers-Rich P, Hall M. 2012. Theropod Fauna from Southern Australia Indicates High Polar Diversity and Climate-Driven Dinosaur Provinciality. PLOS ONE 7: e37122.

Berman DS, McIntosh JS. 1978. Skull and relationships of the Upper Jurassic Sauropod Apatosaurus (Reptilia, Saurischia). Carnegie Museum Bulletin 8: 1-35. 
Bonaparte JF. 1986a. Les Dinosaures (Carnosaures, Allosauridés, Sauropodes, Cétiosauridés) du Jurassiquemoyen de Cerro Condor (Chubut, Argentine) (Dinosaurs (carnosaurs, allosaurids, sauropods, cetiosaurids) of the Middle Jurassic of Cerro Cóndor, Chubut, Argentina). Annales de Paléontologie 72: 325-386.

Bonaparte JF. 1986b. The early radiation and phylogenetic relationships of the Jurassic sauropod dinosaurs, based on vertebral anatomy. In: Padian K, ed. The Beginning of the Age of Dinosaurs. Cambridge; Cambridge University Press, 247-258.

Bonaparte JF, Coria RA. 1993. Un nuevo y gigantesco saurópodo titanosaurio de la Formación Río Limay (Albiano-Cenomaniano) de la Provincia del-Neuquen, Argentina. Ameghiniana 30: 271-282.

Bonaparte JF, Mateus O. 1999. A new diplodocid, Dinheirosaurus lourinhanensis gen. et sp. nov., from the Late Jurassic beds of Portugal. Revista del Museo Argentino de Ciencias Naturales "Bernardino Rivadavia" e Instituto Nacional de Investigación de las Ciencias Naturales, Paleontología 5: 13-29.

Bonaparte JF, Heinrich W-D, Wild R. 2000. Review of Janenschia Wild, with the description of a new sauropod from the Tendaguru beds of Tanzania and a discussion on the systematic value of procoelous caudal vertebrae in the Sauropoda. Palaeontographica, Abteilung A 256: 25-76.

Bonaparte JF, González Riga BJ, Apesteguía S. 2006. Ligabuesaurus leanzai gen. et sp. nov. (Dinosauria, Sauropoda), a new titanosaur from the Lohan Cura Formation (Aptian, Lower Cretaceous) of Neuquén, Patagonia, Argentina. Cretaceous Research 27: 364-376.

Bonnan MF. 2000. The presence of a calcaneum in a diplodocid sauropod. Journal of Vertebrate Paleontology 20: 317-323.

Borsuk-Bialynicka M. 1977. A new camarasaurid sauropod Opisthocoelicaudia skarzynskii gen. n., sp. n. from the Upper Cretaceous of Mongolia. Palaeontologica Polonica 37: 5-63.

Britt BB, Scheetz RD, Whiting MF, Wilhite DR. 2017. Moabosaurus utahensis, n. gen., n. sp., a new sauropod from the Early Cretaceous (Aptian) of North America. Contributions from the Museum of Paleontology, University of Michigan 32: 189-243.

Broschinski A. 1999. Ein Lacertilier (Scincomorpha, Paramacellodidae) aus dem Oberen Jura von Tendaguru (Tansania). Mitteilungen aus dem Museum für Naturkunde Berlin, Geowissenschaftliche Reihe 2: 155-158.

Brusatte SL, Benton MJ, Ruta M, Lloyd GT. 2008. The first 50 Myr of dinosaur evolution: macroevolutionary pattern and morphological disparity. Biology Letters 4: 733-736.

Buffetaut E. 2005. A new sauropod dinosaur with prosauropod-like teeth from the Middle Jurassic of Madagascar. Bulletin de la Société Géologique de France 176: 483-489.

Buffetaut E. 2012. An early spinosaurid dinosaur from the Late Jurassic of Tendaguru (Tanzania) and the evolution of the spinosaurid dentition. Oryctos 10: 1-8.

Burnham KP, Anderson DR. 2002. Model selection and multimodel inference: a practical information-theoretic approach, 2nd ed. Springer-Verlag New York, 488 pp.

Bussert R, Heinrich W-D Aberhan M. 2009. The Tendaguru Formation (Late Jurassic to Early Cretaceous, southern Tanzania): definition, palaeoenvironments, and sequence stratigraphy. Fossil Record 12: 141-174.

Calvo JO. 1994. Jaw mechanics in sauropod dinosaurs. Gaia 10: 183-193.

Calvo JO, Salgado L. 1995. Rebbachisaurus tessonei sp. nov. a new Sauropoda from the Albian-Cenomanian of Argentina; new evidence on the origin of the Diplodocidae. Gaia 11: 13-33.

Calvo JO, Porfiri JD, González Riga BJ, Kellner AWA. 2008. Anatomy of Futalognkosaurus dukei Calvo, Porfiri, González Riga \& Kellner, 2007 (Dinosauria, Titanosauridae) from the 
Neuquén Group (Late Cretaceous), Patagonia, Argentina. Arquivos do Museu Nacional, Rio de Janeiro 65: 511-526.

Campione NE, Brink KS, Freedman EA, McGarrity CT, Evans DC. 2013. 'Glishades ericksoni', an indeterminate juvenile hadrosaurid from the Two Medicine Formation of Montana: implications for hadrosauroid diversity in the latest Cretaceous (Campanian-Maastrichtian) of western North America. Palaeobiodiversity and Palaeoenvironments 93: 65-75.

Campos DA, Kellner AWA, Bertini RJ, Santucci RM. 2005. On a titanosaurid (Dinosauria, Sauropoda) vertebral column from the Bauru Group, Late Cretaceous of Brazil. Arquivos do Museu Nacional, Rio de Janeiro 63: 565-593.

Canudo JI, Ruiz-Omeñaca JI, Barco JL, Royo-Torres R. 2002. ¿Saurópodos asiáticos en el Barremiense inferior (Cretácico Inferior) de España? Ameghiniana 39: 443-452.

Canudo Jl, Royo-Torres R, Cuenca-Bescós G. 2008. A new sauropod: Tastavinsaurus sanzi gen. et sp. nov. from the Early Cretaceous (Aptian) of Spain. Journal of Vertebrate Paleontology 28: 712-731.

Canudo JI, Barco JL, Pereda-Suberbiola X, Ruiz-Omeñaca JI, Salgado L, Torcida Fernández-Baldor F, Gasulla JM. 2009. What Iberian dinosaurs reveal about the bridge said to exist between Gondwana and Laurasia in the Early Cretaceous. Bulletin de la Société Géologique de France 180: 5-11.

Canudo JI, Gasca JM, Moreno-Azanza M, Aurell M. 2012. New information about the stratigraphic position and age of the sauropod Aragosaurus ischiaticus from the Early Cretaceous of the Iberian Peninsula. Geological Magazine 149: 252-263.

Carballido JL, Sander PM. 2014. Postcranial axial skeleton of Europasaurus holgeri (Dinosauria, Sauropoda) from the Upper Jurassic of Germany: implications for sauropod ontogeny and phylogenetic relationships of basal Macronaria. Journal of Systematic Palaeontology 12: 335-387.

Carballido JL, Garrido AC, Canudo JI, Salgado L. 2010. Redescription of Rayososaurus agrioensis Bonaparte (Sauropoda, Diplodocoidea), a rebbachisaurid from the early Late Cretaceous of Neuquén. Geobios 43: 493-502.

Carballido JL, Rauhut OWM, Pol D, Salgado L. 2011. Osteology and phylogenetic relationships of Tehuelchesaurus benitezii (Dinosauria, Sauropoda) from the Upper Jurassic of Patagonia. Zoological Journal of the Linnean Society 163: 605-662.

Carballido JL, Salgado L, Pol D, Canudo JI, Garrido A. 2012. A new basal rebbachisaurid (Sauropoda, Diplodocoidea) from the Early Cretaceous of the Neuquén Basin; evolution and biogeography of the group. Historical Biology 24: 631-654.

Carballido JL, Pol D, Parra Ruge ML, Padilla Bernal S, Páramo-Fonseca ME, Etayo-Serna F. 2015. A new Early Cretaceous brachiosaurid (Dinosauria, Neosauropoda) from northwestern Gondwana (Villa de Leiva, Colombia). Journal of Vertebrate Paleontology 35: e980505.

Carballido JL, Pol D, Otero A, Cerda IA, Salgado L, Garrido AC, Ramezani J, Cúneo NR, Krause JM. 2017. A new giant titanosaur sheds light on body mass evolution among sauropod dinosaurs. Proceedings of the Royal Society of London B 284: 20171219.

Carvalho IS, Avilla LS, Salgado L. 2003. Amazonsaurus maranhensis gen. et sp. nov. (Sauropoda, Diplodocoidea) from the Lower Cretaceous (Aptian-Albian) of Brazil. Cretaceous Research 24: 697-713.

Carvalho IS, Salgado L, Lindoso RM, Araújo-Júnior HID, Nogueira FCC, Soares JA. 2017. A new basal titanosaur (Dinosauria, Sauropoda) from the Lower Cretaceous of Brazil. Journal of South American Earth Sciences 75: 74-84. 
Casanovas ML, Santafe JV, Sanz JL. 2001. Losillasaurus giganteus, un nuevo sauropodo del transito Jurasico-Cretacico de la cuenca de "Los Serranos" (Valencia, España). Paleontologia i Evolucio 32-33: 99-122.

Cerda IA, Salgado L, Powell JE. 2012. Extreme postcranial pneumaticity in sauropod dinosaurs from South America. Paläontologische Zeitschrift 86: 441-449.

Chure D, Britt BB, Whitlock JA, Wilson JA. 2010. First complete sauropod dinosaur skull from the Cretaceous of the Americas and the evolution of sauropod dentition. Naturwissenschaften 97: 379-391.

Cohen KM, Finney SC, Gibbard PL, Fan J-X. 2013. The ICS International Chronostratigraphic Chart. Episodes 36: 199-204.

Cooper MR. 1981. The prosauropod dinosaur Massospondylus carinatus Owen from Zimbabwe: its biology, mode of life and phylogenetic significance. Occasional Papers of the National Museums and Monuments of Rhodesia, Series B, Natural Sciences 6: 689-840.

Coria RA, Filippi LS, Chiappe LM, García R, Arcucci AB. 2013. Overosaurus paradasorum gen. et sp. nov., a new sauropod dinosaur (Titanosauria: Lithostrotia) from the Late Cretaceous of Neuquén, Patagonia, Argentina. Zootaxa 3683: 357-376.

Costa FR, Kellner AWA. 2009. On two pterosaur humeri from the Tendaguru beds (Upper Jurassic, Tanzania). Anais da Academia Brasileira de Ciências 81: 813-818.

Cúneo R, Ramezani J, Scasso R, Pol D, Escapa I, Zavattieri AM, Bowring SA. 2013. Highprecision $\mathrm{U}-\mathrm{Pb}$ geochronology and a new chronostratigraphy for the Cañadón Asfalto Basin, Chubut, central Patagonia: Implications for terrestrial faunal and floral evolution in Jurassic. Gondwana Research 24: 1267-1275.

Currie PJ, Wilson JA, Fanti F, Mainbayar B, Tsogtbaatar K. 2018. Rediscovery of the type localities of the Late Cretaceous Mongolian sauropods Nemegtosaurus mongoliensis and Opisthocoelicaudia skarzynskii: Stratigraphic and taxonomic implications. Palaeogeography, Palaeoclimatology, Palaeoecology 494: 5-13.

Curry Rogers K. 2005. Titanosauria: a phylogenetic overview. In: Curry Rogers K, Wilson JA, eds. The Sauropods: Evolution and Paleobiology. Berkeley; Los Angeles: University of California Press, 50-103.

Curry Rogers K. 2009. The postcranial osteology of Rapetosaurus krausei (Sauropoda: Titanosauria) from the Late Cretaceous of Madagascar. Journal of Vertebrate Paleontology 29: 1046-1086.

Curry Rogers K, Forster CA. 2001. The last of the dinosaur titans: a new sauropod from Madagascar. Nature 412: 530-534.

Curry Rogers K, Forster C. 2004. The skull of Rapetosaurus krausei (Sauropoda: Titanosauria) from the Late Cretaceous of Madagascar. Journal of Vertebrate Paleontology 24: 121-144.

Curtice BD, Stadtman KL. 2001. The demise of Dystylosaurus edwini and a revision of Supersaurus vivianae. Mesa Southwest Museum Bulletin 8: 33-40.

Dal Sasso C, Pierangelini G, Famiani F, Cau A, Nicosia U. 2016. First sauropod bones from Italy offer new insights on the radiation of Titanosauria between Africa and Europe. Cretaceous Research 64: 88-109.

Dalla Vecchia FM. 1998. Remains of Sauropoda (Reptilia, Saurischia) in the lower Cretaceous (upper Hauterivian/lower Barremian) limestones of SW Istria (Croatia). Geologia Croatica 51: 105-134.

Dalla Vecchia FM. 1999. Atlas of the sauropod bones from the upper Hauterivian-lower Barremian of Valle/Bale (SW Istria, Croatia). Natura Nacosta 18: 6-41. 
Dalla Vecchia FM. 2005. Between Gondwana and Laurasia: Cretaceous sauropods in an intraoceanic carbonate platform. In: Tidwell V, Carpenter $\mathrm{K}$, eds. Thunder-lizards: the sauropodomorph dinosaurs. Bloomington, Indianapolis: Indiana University Press, 395-429.

D'Emic MD. 2012. The early evolution of titanosauriform sauropod dinosaurs. Zoological Journal of the Linnean Society 166: 624-671.

D'Emic MD. 2013. Revision of the sauropod dinosaurs of the Lower Cretaceous Trinity Group, southern USA, with the description of a new genus. Journal of Systematic Palaeontology 11: 707-726

D'Emic MD, Foreman BZ. 2012. The beginning of the sauropod dinosaur hiatus in North America: insights from the Lower Cretaceous Cloverly Formation of Wyoming. Journal of Vertebrate Paleontology 32: 883-902.

D'Emic MD, Foster JR. 2016. The oldest Cretaceous North American sauropod dinosaur. Historical Biology 28: 470-478.

D'Emic MD, Wilson JA. 2011. New remains attributable to the holotype of Neuquensaurus australis (Dinosauria: Sauropoda): implications for saltasaurine systematics. Acta Palaeontologia Polonica 56: 61-73.

D'Emic MD, Wilson JA, Williamson TE. 2011. A sauropod dinosaur pes from the latest Cretaceous of North America and the validity of Alamosaurus sanjuanensis (Sauropoda, Titanosauria). Journal of Vertebrate Paleontology 31: 1072-1079.

D’Emic MD, Mannion PD, Upchurch P, Benson RBJ, Pang Q, Cheng Z. 2013. Osteology of Huabeisaurus allocotus (Sauropoda: Titanosauriformes) from the Upper Cretaceous of China. PLOS ONE 8: e69375.

D'Emic MD, Foreman BZ, Jud NA. 2016. Anatomy, systematics, paleoenvironment, growth, and age of the sauropod dinosaur Sonorasaurus thompsoni from the Cretaceous of Arizona, USA. Journal of Paleontology 90: 102-132.

Dong Z. 1990. On remains of the sauropods from Kelamaili region, Junggar Basin, Xinjiang, China. Vertebrata PalAsiatica 28: 43-58.

Ezcurra MD, Agnolín FL. 2012. A new global palaeobiogeographical model for the late Mesozoic and early Tertiary. Systematic Biology 61: 553-566.

Fanti F, Cau A, Hassine M, Contessi M. 2013. A new sauropod dinosaur from the Early Cretaceous of Tunisia with extreme avian-like pneumatization. Nature Communications 4: 1-7.

Fanti F, Cau A, Cantelli L, Hassine M, Auditore M. 2015. New information on Tataouinea hannibalis from the Early Cretaceous of Tunisia and implications for the tempo and mode of rebbachisaurid sauropod evolution. PLOS ONE 10: e0123475.

Ford D, Golonka J. 2003. Phanerozoic paleogeography, paleoenvironment and lithofacies maps of the circum-Atlantic margins. Marine and Petroleum Geology 20: 249-285.

Fraas E. 1908. Ostafrikanische Dinosaurier. Palaeontographica 55: 105-144.

Gaetano LC, Rougier GW. 2012. First Amphilestid from South America: A Molariform from the Jurassic Cañadón Asfalto Formation, Patagonia, Argentina. Journal of Mammalian Evolution 19: 235-248.

Gallina PA. 2011. Notes on the axial skeleton of the titanosaur Bonitasaura salgadoi (Dinosauria-Sauropoda). Anais da Academia Brasileira de Ciências 83: 235-245.

Gallina PA. 2016. Reappraisal of the Early Cretaceous sauropod dinosaur Amargatitanis macni (Apesteguía, 2007), from northwestern Patagonia, Argentina. Cretaceous Research 64: 79-87. 
Gallina PA, Apesteguía S. 2005. Cathartesaura anaerobica gen. et sp. nov., a new rebbachisaurid (Dinosauria, Sauropoda) from the Huincul Formation (Upper Cretaceous), Río Negro, Argentina. Revista del Museo Argentino de Ciencias Naturales n.s. 7: 153-166.

Gallina PA, Apesteguía S. 2011. Cranial anatomy and phylogenetic position of the titanosaurian sauropod Bonitasaura salgadoi. Acta Palaeontologica Polonica 56: 45-60.

Gallina PA, Apesteguía S, Haluza A, Canale JI. 2014. A diplodocid sauropod survivor from the Early Cretaceous of South America. PLoS ONE 9: e97128.

Galton PM. 1977. The ornithopod dinosaur Dryosaurus and a Laurasia-Gondwanaland connection in the Upper Jurassic. Nature 268: 230-232.

Galton PM. 1982. The postcranial anatomy of stegosaurian dinosaur Kentrosaurus from the Upper Jurassic of Tanzania, East Africa. Geologica et Palaeontologica 15: 139-160.

Gauthier J. 1986. Saurischian monophyly and the origin of birds. Memoirs of the Californian Academy of Sciences 8: 1-55.

Gheerbrant E, Rage J-C. 2006. Paleobiogeography of Africa: how distinct from Gondwana and Laurasia? Palaeogeography, Palaeoclimatology, Palaeoecology 241: 224-246.

Ghilardi AM, Aureliano T, Duque RRC, Fernandes MA, Barreto AMF, Chinsamy A. 2016. A new titanosaur from the Lower Cretaceous of Brazil. Cretaceous Research 67: 16-24.

Gilmore CW. 1932. On a newly mounted skeleton of Diplodocus in the United States National Museum. Proceedings of the United States National Museum 81: 1-21.

Gilmore CW. 1936. Osteology of Apatosaurus with special reference to specimens in the Carnegie Museum. Memoirs of the Carnegie Museum 11: 175-300.

Gnaedinger S, Coria RA, Koppelhus E, Casadío S, Tunik M, Currie P. 2017. First Lower Cretaceous record of Podocarpaceae wood associated with dinosaur remains from Patagonia, Neuquén Province, Argentina. Cretaceous Research 78: 228-239.

Goloboff PA. 2014. Extended implied weighting. Cladistics 30: 260-272.

Goloboff PA, Catalano SA. 2016. TNT version 1.5, including a full implementation of phylogenetic morphometrics. Cladistics 32: 221-238.

Goloboff PA, Farris JS, Nixon KC. 2008. TNT, a free program for phylogenetic analysis. Cladistics 24: 1-13.

Gomani EM. 2005. Sauropod dinosaurs from the Early Cretaceous of Malawi. Palaeontologia Electronica 8: 1-37.

González Riga BJ. 2003. A new titanosaur (Dinosauria, Sauropoda) from the Upper Cretaceous of Mendoza Province, Argentina. Ameghiniana 40: 155-172.

González Riga BJ. 2005. Nuevos restos fósiles de Mendozasaurus neguyelap (Sauropoda, Titanosauridae) del Cretácico tardío de Patagonia. Ameghiniana 43: 535-548.

González Riga BJ, Ortiz David L. 2014. A New Titanosaur (Dinosauria, Sauropoda) from the Upper Cretaceous (Cerro Lisandro Formation) of Mendoza Province, Argentina. Ameghiniana 51: 3-25.

González Riga BJ, Calvo JO, Porfiri J. 2008. An articulated titanosaur from Patagonia (Argentina): new evidence of neosauropod pedal evolution. Palaeoworld 17: 33-40.

González Riga BJ, Previtera E, Pirrone CA. 2009. Malarguesaurus florenciae gen. et sp. nov., a new titanosauriform (Dinosauria, Sauropoda) from the Upper Cretaceous of Mendoza, Argentina. Cretaceous Research 30: 135-148.

González Riga BJ, Lamanna MC, Ortiz David LD, Calvo JO, Coria JP. 2016. A gigantic new dinosaur from Argentina and the evolution of the sauropod hind foot. Scientific Reports 6: 19165.

González Riga BJ, Mannion PD, Poropat SF, Ortiz David L, Coria JP. 2018. Osteology of the Late Cretaceous Argentinean sauropod dinosaur Mendozasaurus neguyelap: implications 
for basal titanosaur relationships. Zoological Journal of the Linnean Society (doi: 10.1093/zoolinnean/zlx103).

Gorscak E, O'Connor PM. 2016. Time-calibrated models support congruency between Cretaceous continental rifting and titanosaurian evolutionary history. Biology Letters 12: 20151047.

Graham SA, Hendrix MS, Barsbold R, Badamgarav D, Sjostrom D, Kirschner W, McIntosh JS. 1997. Stratigraphic occurrence, paleoenvironment, and description of the oldest known dinosaur (Late Jurassic) from Mongolia. Palaios 12: 292-297.

Haluza A, Canale JI, Otero A, Peréz LM, Scanferla CA. 2012. Changes in vertebral laminae across the cervicodorsal transition of a well-preserved rebbachisaurid (Dinosauria, Sauropoda) from the Cenomanian of Patagonia, Argentina. Journal of Vertebrate Paleontology 32: 219-224.

Harris JD. 2006a. The axial skeleton of the dinosaur Suuwassea emilieae (Sauropoda: Flagellicaudata) from the Upper Jurassic Morrison Formation of Montana, USA. Palaeontology 49: 1091-1121.

Harris JD. 2006b. The significance of Suuwassea emilieae (Dinosauria: Sauropoda) for flagellicaudatan intrarelationships and evolution. Journal of Systematic Palaeontology 4: 185-198.

Harris JD. 2006c. Cranial osteology of Suuwassea emilieae (Sauropoda: Diplodocoidea: Flagellicaudata) from the Upper Jurassic Morrison Formation of Montana, USA. Journal of Vertebrate Paleontology 26: 88-102.

Harris JD. 2007. The appendicular skeleton of Suuwassea emilieae (Sauropoda: Flagellicaudata) from the Upper Jurassic Morrison Formation of Montana (USA). Geobios 40: 501-522.

Harris JD, Dodson P. 2004. A new diplodocoid sauropod dinosaur from the Upper Jurassic Morrison Formation of Montana, USA. Acta Palaeontologica Polonica 49: 197-210.

Hasegawa Y, Manabe M, Hanai T, Kase T, Oji T. 1991. A diplodocoid dinosaur from the Early Cretaceous Miyako Group of Japan. Bulletin of the National Science Museum, Tokyo, Series C 17: 1-9.

Hatcher JB. 1901. Diplodocus (Marsh): Its osteology, taxonomy, and probable habits, with a restoration of the skeleton. Memoirs of the Carnegie Museum 1: 1-63.

Hatcher JB. 1903. Osteology of Haplocanthosaurus, with description of a new species, and remarks on the probable habits of the Sauropoda and the age and origin of the Atlantosaurus beds. Memoirs of the Carnegie Museum 2: 1-72.

Haughton SH. 1928. On some reptilian remains from the Dinosaurian Beds of Nyasaland. Transactions of the Royal Society of South Africa 16: 67-75.

He X-L, Li K, Cai K-J. 1988. The Middle Jurassic Dinosaur Fauna from Dashanpu, Zigong, Sichuan. Vol IV. Sauropod Dinosaurs (2). Omeisaurus tianfuensis. Sichuan Publishing House of Science and Technology, Chengdu, China, 143 pp. [In Chinese, English summary].

Heinrich W-D. 1998. Late Jurassic mammals from Tendaguru, Tanzania, East Africa. Journal of Mammalian Evolution 5: 269-290.

Heinrich W-D. 1999a. First haramiyid (Mammalia, Allotheria) from the Mesozoic of Gondwana. Mitteilungen aus dem Museum für Naturkunde Berlin, Geowissenschaftliche Reihe 2: 159-170.

Heinrich W-D. 1999b. The taphonomy of dinosaurs from the Upper Jurassic of Tendaguru (Tanzania) based on field sketches of the German Tendaguru Expedition (19091913). Mitteilungen aus dem Museum für Naturkunde in Berlin, Geowissenschaften Reihe 2: 25-61. 
Heinrich W-D. 2001. New records of Staffia aenigmatica (Mammalia, Allotheria, Haramiyida) from the Upper Jurassic of Tendaguru in southeastern Tanzania, East Africa. Mitteilungen aus dem Museum für Naturkunde in Berlin, Geowissenschaftliche Reihe 4: 239-255.

Heinrich W-D, Bussert R, Aberhan M, Hampe O, Kapilima S, Schrank E, Schultka S, Maier G, Msaky E, Sames B, Chami R. 2001. The German-Tanzanian Tendaguru Expedition 2000. Mitteilungen aus dem Museum für Naturkunde in Berlin, Geowissenschaftliche Reihe 4: 223-237.

Hennig E. 1915. Kentrosaurus aethiopicus, der Stegosauridae des Tendaguru. Sitzungsberichte der Gesellschaft Naturforschender Freunde zu Berlin 1915: 219-247.

Holwerda FM, Pol D, Rauhut OWM. 2015. Using dental enamel wrinkling to define sauropod tooth morphotypes from the Cañadón Asfalto Formation, Patagonia, Argentina. PLoS ONE 10: e0118100.

Huang J-D, You H-L, Yang J-T, Ren X-X. 2014. A new sauropod dinosaur from the Middle Jurassic of Huangshan, Anhui Province. Vertebrata PalAsiatica 52: 390-400.

Hübner TR, Rauhut OWM. 2010. A juvenile skull of Dysalotosaurus lettowvorbecki (Ornithischia: Iguanodontia), and implications for cranial ontogeny, phylogeny, and taxonomy in ornithopod dinosaurs. Zoological Journal of the Linnean Society 160: 366-396.

Ibiricu LM, Casal GM, Lamanna MC, Martínez RD, Harris JD, Lacovara KJ. 2012. The southernmost records of Rebbachisauridae (Sauropoda: Diplodocoidea), from early Late Cretaceous deposits in central Patagonia. Cretaceous Research 34: 220-232.

Ibiricu LM, Casal GA, Martínez RD, Lamanna MC, Luna M, Salgado L. 2013. Katepensaurus goicoecheai, gen. et sp. nov., a Late Cretaceous rebbachisaurid (Sauropoda, Diplodocoidea) from central Patagonia, Argentina. Journal of Vertebrate Paleontology 33: 1351-1366.

Ibiricu LM, Casal GA, Martínez RD, Lamanna MC, Luna M, Salgado L. 2015. New material of Katepensaurus goicoecheai (Sauropoda: Diplodocoidea) and its significance for the morphology and evolution of Rebbachisauridae. Ameghiniana 52: 430-446.

Iturralde-Vinent M. 2006. Meso-Cenozoic Caribbean paleogeography: implications for the historical biogeography of the region. International Geology Review 48: 791-827.

Jacobs LL, Winkler DA, Downs WR, Gomani EM. 1993. New material of an Early Cretaceous titanosaurid sauropod dinosaur from Malawi. Palaeontology 36: 523-534.

Janensch W. 1914a. Übersicht über der Wirbeltierfauna der Tendaguru-Schichten nebst einer kurzen Charakterisierung der neu aufgefuhrten Arten von Sauropoden. Archiv fur Biontologie 3: 81-110.

Janensch W. 1914b. Bericht über den Verlauf der Tendaguru-Expedition. Archiv fur Biontologie 3: 17-58.

Janensch W. 1920. Ueber Elaphrosaurus bambergi und die Megalosaurier aus den Tendaguru-Schichten Deutsch-Ostafrikas. Sitzungsberichte der Gesellschaft Naturforschender Freunde zu Berlin 1920: 225-235.

Janensch W. 1922. Das Handskelett von Gigantosaurus robustus u. Brachiosaurus Brancai aus den Tendaguru-Schichten Deutsch-Ostafrikas. Centralblatt für Mineralogie, Geologie und Paläontologie 15: 464-480.

Janensch W. 1925a. Die Coelurosaurier und Theropoden der Tendaguru-Schichten DeutschOstafrikas. Palaeontographica (Supplement VII) 1: 1-100.

Janensch W. 1925b. Die Grabungsstellen der Tendaguru-Gegend. Palaeontographica (Supplement VII) 1: 17-19.

Janensch W. 1929a. Material und Formegehalt der Sauropoden in der Ausbeute der Tendaguru-Expedition, 1909-1912. Palaeontographica (Supplement VII) 2: 3-34. 
Janensch W. 1929b. Die Wirbelsäule der Gattung Dicraeosaurus. Palaeontographica (Supplement VII) 2: 37-133.

Janensch W. 1950. Die wirbelsäule von Brachiosaurus brancai. Palaeontographica (Supplement VII) 3: 27-93.

Janensch W. 1961. Die Gliedmaszen und Gliedmaszengürtel der Sauropoden der TendaguruSchichten. Palaeontographica (Supplement VII) 3: 177-235.

Jensen JA. 1985. Three new sauropod dinosaurs from the Upper Jurassic of Colorado. The Great Basin Naturalist 45: 697-709.

Kellner AWA, Campos DA, Trotta MNF. 2005. Description of a titanosaurid caudal series from the Bauru Group, Late Cretaceous of Brazil. Arquivos do Museu Nacional, Rio de Janeiro 63: 529-564.

Ksepka DT, Norell MA. 2010. The illusory evidence for Asian Brachiosauridae: new material of Erketu ellisoni and a phylogenetic reappraisal of basal Titanosauriformes. American Museum Novitates 3700: 1-27.

Läng E, Mahammed F. 2010. New anatomical data and phylogenetic relationships of Chebsaurus algeriensis (Dinosauria, Sauropoda) from the Middle Jurassic of Algeria. Historical Biology 22: 142-164.

Lapparent AF. 1955. Étude paléontologique des vertébrés du Jurassique d'El Mers (Moyen Atlas). Notes et Mémoires du Service Géologique du Maroc 124: 1-36.

Lapparent AF. 1960. Les dinosauriens du "Continental Intercalaire" du Sahara central. Mémoires de la Société Géologique de France 88A: 1-56.

Li K, Yang C-Y, Liu J, Wang Z-X. 2010. A new sauropod from the Lower Jurassic of Huili, Sichuan, China. Vertebrata PalAsiatica 48: 185-202.

Loewen MA, Irmis RB, Sertich JJW, Currie PJ, Sampson SD. 2013. Tyrant dinosaur evolution tracks the rise and fall of Late Cretaceous oceans. PLOS ONE 8: e79420.

Lovelace DM, Hartman SA, Wahl WR. 2008. Morphology of a specimen of Supersaurus (Dinosauria, Sauropoda) from the Morrison Formation of Wyoming, and a re-evaluation of diplodocoid phylogeny. Arquivos do Museu Nacional, Rio de Janeiro 65: 527-544.

Lü J, Li T, Zhong S, Ji Q, Li S. 2008. A new mamenchisaurid dinosaur from the Middle Jurassic of Yuanmou, Yunnan Province, China. Acta Geologica Sinica 82: 17-26.

Lü J, Xu L, Zhang X, Hu W, Wu Y, Jia S, Ji Q. 2007. A new gigantic sauropod dinosaur with the deepest known body cavity from the Cretaceous of Asia. Acta Geologica Sinica 81: 167176.

Lü J, Xu L, Jiang X, Jia S, Li M, Yuan C, Zhang X, Ji Q. 2009. A preliminary report on the new dinosaurian fauna from the Cretaceous of the Ruyang Basin, Henan Province of Central China. Journal of the Paleontological Society of Korea 25: 43-56.

Lydekker R. 1888. Catalogue of the Fossil Reptilia and Amphibia in the British Museum (Natural History). Part I. Containing the Orders Ornithosauria, Crocodilia, Dinosauria, Squamata, Rhynchocephalia, and Proterosauria. British Museum (Natural History): London, 1-309.

Madsen J, McIntosh JS, Berman DS. 1995. Skull and atlas-axis complex of the Upper Jurassic sauropod Camarasaurus Cope (Reptilia: Saurischia). Bulletin of the Carnegie Museum of Natural History 31: 1-115.

Mahammed F, Läng E, Mami L, Mekahli L, Benhamou M, Bouterfa B, Kacemi A, Chérief S, Chaouati H, Taquet P. 2005. The "Giant of Ksour", a Middle Jurassic sauropod dinosaur from Algeria. Comptes Rendus Palevol 4: 707-714.

Maier G. 2003. African Dinosaurs Unearthed: The Tendaguru Expeditions. Indiana University Press, Bloomington, $380 \mathrm{pp}$. 
Manning PL, Egerton VM, Romano M. 2015. A New Sauropod Dinosaur from the Middle Jurassic of the United Kingdom. PLOS ONE 10: e0128107.

Mannion PD. 2009. A rebbachisaurid sauropod from the lower Cretaceous of the Isle of Wight, England. Cretaceous Research 30: 521-526.

Mannion PD. 2010. A revision of the sauropod dinosaur genus "Bothriospondylus" with a redescription of the type material of the Middle Jurassic form "B. madagascariensis". Palaeontology 53: 277-296.

Mannion PD. 2011. A reassessment of Mongolosaurus haplodon Gilmore, 1933, a titanosaurian sauropod dinosaur from the Early Cretaceous of Inner Mongolia, People's Republic of China. Journal of Systematic Palaeontology 9: 355-378.

Mannion PD, Barrett PM. 2013. Additions to the sauropod dinosaur fauna of the Cenomanian (early Late Cretaceous) Kem Kem beds of Morocco: palaeobiogeographical implications of the mid-Cretaceous African sauropod fossil record. Cretaceous Research 45: 49-59.

Mannion PD, Calvo JO. 2011. Anatomy of the basal titanosaur (Dinosauria, Sauropoda) Andesaurus delgadoi from the mid-Cretaceous (Albian-early Cenomanian) Río Limay Formation, Neuquén Province, Argentina: implications for titanosaur systematics. Zoological Journal of the Linnean Society 163: 155-181.

Mannion PD, Otero A. 2012. A reappraisal of the Late Cretaceous Argentinean sauropod dinosaur Argyrosaurus superbus, with a description of a new titanosaur genus. Journal of Vertebrate Paleontology 32: 614-638.

Mannion PD, Allain R, Moine 0. 2017. The earliest known titanosauriform sauropod dinosaur and the evolution of Brachiosauridae. PeerJ 5: e3217.

Mannion PD, Upchurch P, Hutt S. 2011. New rebbachisaurid (Dinosauria: Sauropoda) material from the Wessex Formation (Barremian, Early Cretaceous), Isle of Wight, United Kingdom. Cretaceous Research 32: 774-780.

Mannion PD, Upchurch P, Mateus O, Barnes R, Jones MEH. 2012. New information on the anatomy and systematic position of Dinheirosaurus lourinhanensis (Sauropoda: Diplodocoidea) from the Late Jurassic of Portugal, with a review of European diplodocoids. Journal of Systematic Palaeontology 10: 521-551.

Mannion PD, Upchurch P, Barnes RN, Mateus O. 2013. Osteology of the Late Jurassic Portuguese sauropod dinosaur Lusotitan atalaiensis (Macronaria) and the evolutionary history of basal titanosauriforms. Zoological Journal of the Linnean Society 168: 98-206.

Marpmann JS, Carballido JL, Sander PM, Knötschke N. 2015. Cranial anatomy of the Late Jurassic dwarf sauropod Europasaurus holgeri(Dinosauria, Camarasauromorpha): ontogenetic changes and size dimorphism. Journal of Systematic Palaeontology 13: 221263.

Marsh OC. 1878. Principal characters of American Jurassic dinosaurs. Part I. American Journal of Science 16: 411-416.

Martínez RD, Giménez O, Rodríguez J, Luna M, Lamanna MC. 2004. An articulated specimen of the basal titanosaurian (Dinosauria: Sauropoda) Epachthosaurus sciuttoi from the early Late Cretaceous Bajo Barreal Formation of Chubut province, Argentina. Journal of Vertebrate Paleontology 24: 107-120.

Mateus 0. 2006. Late Jurassic dinosaurs from the Morrison Formation (USA), the Lourinhã and Alcobaça formations (Portugal), and the Tendaguru Beds (Tanzania): a comparison. New Mexico Museum of Natural History and Science Bulletin 36: 223-231. 
Mateus O, Mannion PD, Upchurch P. 2014. Zby atlanticus, a new turiasaurian sauropod (Dinosauria, Eusauropoda) from the Late Jurassic of Portugal. Journal of Vertebrate Paleontology 34: 618-634.

Matzke NJ. 2013. Probabilistic historical biogeography: new models for founder-event speciation, imperfect detection, and fossils allow improved accuracy and model-testing. Frontiers of Biogeography 5: 242-248.

Matzke NJ. 2014. Model selection in historical biogeography reveals that founder-event speciation is a crucial process in island clades. Systematic Biology 63: 951-970.

McIntosh JS. 1981. Annotated catalogue of the dinosaurs (Reptilia, Archosauria) in the collections of Carnegie Museum of Natural History. Bulletin of Carnegie Museum of Natural History 18: 1-67.

McIntosh JS. 1990. Sauropoda. In: Weishampel DB, Dodson P, Ósmolska H, eds. The Dinosauria. First edn. Berkeley, CA: University California Press, 345-401.

McIntosh JS. 2005. The genus Barosaurus Marsh (Sauropoda, Diplodocidae). In: Tidwell V, Carpenter K, eds. Thunder-lizards: the sauropodomorph dinosaurs. Bloomington, Indianapolis: Indiana University Press, 38-77.

McIntosh JS, Williams ME. 1988. A new species of sauropod dinosaur, Haplocanthosaurus delfsi sp. nov., from the Upper Jurassic Morrison Formation of Colorado. Kirtlandia 43: 326.

McIntosh JS, Coombs WP, Russell DA. 1992. A new diplodocid sauropod (Dinosauria) from Wyoming, U.S.A. Journal of Vertebrate Paleontology 12: 158-167.

McIntosh JS, Miller WE, Stadtman KL, Gillette DD. 1996. The osteology of Camarasaurus lewisi (Jensen, 1988). Brigham Young University Geology Studies 41: 73-115.

McPhee BW, Mannion PD, de Klerk WJ, Choiniere JN. 2016. High diversity in the sauropod dinosaur fauna of the Lower Cretaceous Kirkwood Formation of South Africa: implications for the Jurassic-Cretaceous transition. Cretaceous Research 59: 228-248.

Migeod FWH. 1931. British Museum East Africa Expedition. Account of the work done in 1930. Natural History Magazine 3: 87-103.

Mo J. 2013. Bellusaurus sui. Henan Science and Technology Press, Zhengzhou, China, 154 pp.

Mocho P, Royo-Torres R, Ortega F. 2014. Phylogenetic reassessment of Lourinhasaurus alenquerensis, a basal Macronaria (Sauropoda) from the Upper Jurassic of Portugal. Zoological Journal of the Linnean Society 170: 875-916.

Mocho, P, Royo-Torres R, Malafaia E, Escaso F, Silva B, Ortega F. 2016. Turiasauria-like teeth from the Upper Jurassic of the Lusitanian Basin, Portugal. Historical Biology 28: 861880.

Mocho, P, Royo-Torres R, Malafaia E, Escaso F, Ortega F. 2017a. First occurrences of nonneosauropod eusauropod procoelous caudal vertebrae in the Portuguese Upper Jurassic record. Geobios 50: 23-36.

Mocho P, Royo-Torres R, Ortega F. 2017b. New data of the Portuguese brachiosaurid Lusotitan atalaiensis (Sobral Formation, Upper Jurassic). Historical Biology 29: 789-817.

Moore AJ, Mo J, Clark J, Xu X. 2015. New cranial material of Bellusaurus sui (Dinosauria: Sauropoda) from the Middle-Late Jurassic Shishugou Formation of China supports neosauropod affinities. Journal of Vertebrate Paleontology (Supplement) 35: 183A.

Moore AJ, Xu X, Clark J. 2017. Anatomy and systematics of Klamelisaurus gobiensis, a mamenchisaurid sauropod from the Middle-Late Jurassic Shishugou Formation of China. Journal of Vertebrate Paleontology (Supplement) 37: 165A. 
Nair JP, Salisbury SW. 2012. New anatomical information on Rhoetosaurus brownei Longman, 1926, a gravisaurian sauropodomorph dinosaur from the Middle Jurassic of Queensland, Australia. Journal of Vertebrate Paleontology 32: 369-394.

Naish D, Martill DM. 2001. Saurischian dinosaurs 1: Sauropods. In: Martill DM, Naish D, eds. Dinosaurs of the Isle of Wight. London: Palaeontological Association, 185-241.

Naish D, Martill DM. 2007. Dinosaurs of Great Britain and the role of the Geological Society of London in their discovery: basal Dinosauria and Saurischia. Journal of the Geological Society, London 164: 493-510.

Néraudeau $D$, Allain R, Ballèvre $M$, Batten DJ, Buffetaut $E$, Colin JP, Dabard MP, DavieroGomez V, El Albani A, Gomez B, Grosheny D, Le Loeuff J, Leprince A, Martín-Closas C, Masure E, Mazin J-M, Phillipe M, Pouech J, Tong H, Tournepiche JF, Vullo R. 2012. The Hautevarian-Barremian lignitic bone bed of Angeac (Charente, south-west France): stratigraphical, palaeobiological and palaeogeographical implications. Cretaceous Research 37: 1-14.

Nicholl CSC, Mannion PD, Barrett PM. 2018. Sauropod dinosaur remains from a new Early Jurassic locality in the Central High Atlas of Morocco. Acta Palaeontologica Polonica 63: 147-157.

Nopcsa F. 1902. Notizen uber Cretacischen Dinosaurier. Pt. 3. Wirbel eines sudamerikanischen Sauropoden. Akademie der Wissenschaften 3: 108-114.

Novas FE, Salgado L, Calvo J \& Agnolin F. 2005. Giant titanosaur (Dinosauria, Sauropoda) from the Late Cretaceous of Patagonia. Revisto del Museo Argentino de Ciencias Naturales, n.s. 7: 37-41.

Osborn HF, Mook CC. 1921. Camarasaurus, Amphicoelias, and other sauropods of Cope. Memoirs of the American Museum of Natural History New Series 3: 247-387.

Otero A. 2010. The appendicular skeleton of Neuquensaurus, a Late Cretaceous saltasaurine sauropod from Patagonia, Argentina. Acta Palaeontologica Polonica 55: 399-426.

Ouyang H, Ye Y. 2002. The first mamenchisaurian skeleton with complete skull: Mamenchisaurus youngi. Sichuan Science and Technology Press, Chengdu: 1-111.

Parrish J. 1993. Climate of the Supercontinent Pangea. Journal of Geology 101: 215-233.

Paulina Carabajal A. 2012. Neuroanatomy of titanosaurid dinosaurs from the Upper Cretaceous of Patagonia, with comments on endocranial variability within Sauropoda. The Anatomical Record 295: 2141-2156.

Paulina Carabajal A, Coria RA, Currie PJ, Koppelhus EB. 2018. A natural cranial endocast with possible dicraeosaurid (Sauropoda, Diplodocoidea) affinities from the Lower Cretaceous of Patagonia. Cretaceous Research 84: 437-441.

Pereda-Suberbiola X, Torcida F, Izquierdo LA, Huerta P, Montero D, Perez G. 2003. First rebbachisaurid dinosaur (Sauropoda, Diplodocoidea) from the early Cretaceous of Spain: palaeobiogeographical implications. Bulletin de la Societie Géologiques de France 174: 471-479.

Peters SE, Heim NA. 2010. The geological completeness of paleontological sampling in North America. Paleobiology 36: 61-79.

Pindell JL, Kennan L. 2009. Tectonic evolution of the Gulf of Mexico, Caribbean and northern South America in the mantle reference frame: an update. Geological Society, London, Special Publications 328: 1-55.

Pol D, Rauhut OWM. 2012. A Middle Jurassic abelisaurid from Patagonia and the early diversification of theropod dinosaurs. Proceedings of the Royal Society London B 279: 3170-3175. 
Pol D, Rauhut OWM, Becerra M. 2011. A Middle Jurassic heterodontosaurid dinosaur from Patagonia and the evolution of heterodontosaurids. Naturwissenschaften 98: 369-379.

Pol D, Rauhut O, Carballido JL. 2009. Skull anatomy of a new basal eusauropod from the Middle Jurassic of Patagonia. Journal of Vertebrate Paleontology 29 (supplement to no. 3): 165 A.

Poropat SF, Upchurch P, Mannion PD, Hocknull SA, Kear BP, Sloan T, Sinapius GHK, Elliott DA. 2015a. Revision of the sauropod dinosaur Diamantinasaurus matildae Hocknull et al. 2009 from the middle Cretaceous of Australia: implications for Gondwanan titanosauriform dispersal. Gondwana Research 27: 995-1033.

Poropat SF, Mannion PD, Upchurch P, Hocknull SA, Kear BP, Elliott DA. 2015b. Reassessment of the non-titanosaurian somphospondylan Wintonotitan wattsi (Dinosauria: Sauropoda: Titanosauriformes) from the mid-Cretaceous Winton Formation, Queensland, Australia. Papers in Palaeontology 1: 59-106.

Poropat SF, Mannion PD, Upchurch P, Hocknull SA, Kear BP, Kundrát M, Tischler TT, Sloan T, Sinapius GHK, Elliott JA, Elliott DA. 2016. New Australian sauropods shed light on Cretaceous dinosaur palaeobiogeography. Scientific Reports 6:34467.

Poropat SF, Nair JP, Syme CE, Mannion PD, Upchurch P, Hocknull SA, Cook AG, Tischler TR, Holland T. 2017. Reappraisal of Austrosaurus mckillopi Longman, 1933 from the Allaru Mudstone of Queensland, Australia's first named Cretaceous sauropod dinosaur. Alcheringa 41: 543-580.

Powell JE. 1986. Revision de los Titanosauridos de America del Sur. Unpublished Ph.D. dissertation, Universidad Nacional de Tucumán, Tucumán, Argentina.

Powell JE. 1992. Osteologia de Saltasaurus loricatus (Sauropoda-Titanosauridae) del Cretacico Superior del Noroeste argentino. In: Sanz JL, Buscalioni AD, eds. Los dinosaurios y su entorno biotico. Cuenca: Instituto Juan de Valdes, Serie Actas Academicas, 165-230.

Powell JE. 2003. Revision of South American titanosaurid dinosaurs: palaeobiological, palaeobiogeographical and phylogenetic aspects. Records of the Queen Victoria Museum 111: $1-173$.

R CORE TEAM. 2015. R: A language and environment for statistical computing, v. 3.3.2. $R$ Foundation for Statistical Computing, Vienna, Austria (http://www.R-project.org/).

Raath MA, McIntosh JS. 1987. Sauropod dinosaurs from the central Zambesi valley, Zimbabwe, and the age of the Kadzi Formation. South African Journal of Geology 90: 107119.

Rauhut OWM. 2003. A dentary of Patagosaurus (Sauropoda) from the Middle Jurassic of Patagonia. Ameghiniana 40: 425-432.

Rauhut OWM. 2005. Post-cranial remains of 'coelurosaurs' (Dinosauria, Theropoda) from the Late Jurassic of Tanzania. Geological Magazine 142: 97-107.

Rauhut OWM. 2006. A brachiosaurid sauropod from the Late Jurassic Cañadón Calcàreo Formation of Chubut, Argentina. Fossil Record 9: 226-237.

Rauhut OWM. 2011. Theropod dinosaurs from the Late Jurassic of Tendaguru (Tanzania). Special Papers in Palaeontology 86: 195-239.

Rauhut OWM, López-Arbarello A. 2008. Archosaur evolution during the Jurassic: a southern perspective. Revista de la Asociación Geológica Argentina 63: 557-585.

Rauhut OWM, López-Arbarello A. 2009. Considerations on the age of the Tiouaren Formation (Iullemmeden Basin, Niger, Africa): Implications for Gondwanan Mesozoic terrestrial vertebrate faunas. Palaeogeography, Palaeoclimatology, Palaeoecology 271: 259-267. 
Rauhut OWM, Carballido JL, Pol D. 2015. A diplodocid sauropod dinosaur from the Late Jurassic Cañadón Calcáreo Formation of Chubut, Argentina. Journal of Vertebrate Paleontology 35: e982798.

Rauhut OWM, Carrano MT. 2016. The theropod dinosaur Elaphrosaurus bambergi Janensch, 1920, from the Late Jurassic of Tendaguru, Tanzania. Zoological Journal of the Linnean Society 178: 546-610.

Rauhut OWM, Remes K, Fechner R, Cladera G, Puerta P. 2005. Discovery of a short-necked sauropod dinosaur from the Late Jurassic period of Patagonia. Nature 435: 670-672.

Remes K. 2006. Revision of the Tendaguru sauropod Tornieria africana (Fraas) and its relevance for sauropod paleobiogeography. Journal of Vertebrate Paleontology 26: 651669.

Remes K. 2007. A second Gondwanan diplodocid dinosaur from the Upper Jurassic Tendaguru Beds of Tanzania, East Africa. Palaeontology 50: 653-667.

Remes K. 2009. Taxonomy of Late Jurassic diplodocid sauropods from Tendaguru (Tanzania). Fossil Record 12: 23-46.

Remes K, Ortega F, Fierro I, Joger U, Kosma R, Ferrer JMM, PALDES, SNHM, Ide OA, Maga A. 2009. A new basal sauropod dinosaur from the Middle Jurassic of Niger and the early evolution of Sauropoda. PLOS ONE 4: e6924.

Rose PJ. 2007. A new titanosauriform sauropod (Dinosauria: Saurischia) from the Early Cretaceous of central Texas and its phylogenetic relationships. Palaeontologica Electronica 10: $1-65$.

Royo-Torres R. 2009. El saurópodo de Peñarroya de Tastavins. Instituto de Estudios Turolenses-Fundación Conjunto Paleontológico de Teruel-Dinópolis, Monografías Turolenses 6: 1-548.

Royo-Torres R, Alcalá L, Cobos A. 2012. A new specimen of the Cretaceous sauropod Tastavinsaurus sanzi from El Castellar (Teruel, Spain), and a phylogenetic analysis of the Laurasiformes. Cretaceous Research 34: 61-83.

Royo-Torres R, Cobos A, Alcalá L. 2006. A giant European dinosaur and a new sauropod clade. Science 314: 1925-1927.

Royo-Torres R, Cobos A. 2009. Turiasaur sauropods in the Tendaguru Beds of Tanzania. Journal of Vertebrate Paleontology 29 (supplement to no. 3): 173A.

Royo-Torres R, Upchurch P. 2012. The cranial anatomy of the sauropod Turiasaurus riodevensis and implications for its phylogenetic relationships. Journal of Systematic Palaeontology 10: 553-583.

Royo-Torres R, Cobos A, Luque L, Aberasturi A, Espílez E, Fierro I, González ANA, Mampel L, Alcalá L. 2009. High European sauropod dinosaur diversity during Jurassic-Cretaceous transition in Riodeva (Teruel, Spain). Palaeontology 52: 1009-1027.

Royo-Torres R, Upchurch P, Mannion PD, Mas R, Cobos A, Gascó F, Alcalá L, Sanz JL. 2014. The anatomy, phylogenetic relationships and stratigraphic position of the TithonianBerriasian Spanish sauropod dinosaur Aragosaurus ischiaticus. Zoological Journal of the Linnean Society 171: 623-655.

Royo-Torres R, Upchurch P, Kirkland JI, DeBlieux DD, Foster JR, Cobos A, Alcalá L. 2017a. Descendants of the Jurassic turiasaurs from Iberia found refuge in the Early Cretaceous of western USA. Scientific Reports 7: 14311.

Royo-Torres R, Fuentes C, Meijide M, Meijide-Fuentes F, Meijide-Fuentes M. 2017b. A new Brachiosauridae Sauropod dinosaur from the lower Cretaceous of Europe (Soria Province, Spain). Cretaceous Research 80: 38-55. 
Russell DA. 1993. The role of central Asia in dinosaurian biogeography. Canadian Journal of Earth Science 30: 2002-2012.

Russell DA, Zheng Z. 1993. A large mamenchisaurid from the Junggar Basin, Xinjiang, People's Republic of China. Canadian Journal of Earth Sciences 30: 2082-2095.

Salgado L, Bonaparte JF. 1991. Un nuevo saurópodo Dicraeosauridae, Amargasaurus cazaui gen. et sp. nov., de la Formacion La Amarga, Neocomiano de la provincia del Neuquen, Argentina. Ameghiniana 28: 333-346.

Salgado L, Calvo JO. 1992. Cranial osteology of Amargasaurus cazaui Salgado and Bonaparte (Sauropoda, Dicraeosauridae) from the Neocomian of Argentina. Ameghiniana 29: 337346.

Salgado L, Calvo JO. 1997. Evolution of titanosaurid sauropods. II: The cranial evidence. Ameghiniana 34: 33-47.

Salgado L, Carvalho IS. 2008. Uberabatitan ribeiroi, a new titanosaur from the Marília Formation (Bauru Group, Upper Cretaceous), Minas Gerais, Brazil. Palaeontology 51: 881901.

Salgado L, Powell JE. 2010. Reassessment of the vertebral laminae in some South American titanosaurian sauropods. Journal of Vertebrate Paleontology 30: 1760-1772.

Salgado L, Apesteguía S, Heredia SE. 2005. A new specimen of Neuquensaurus australis, a Late Cretaceous saltasaurine titanosaur from north Patagonia. Journal of Vertebrate Paleontology 25: 623-634.

Salgado L, Carvalho IS, Garrido AC. 2006. Zapalasaurus bonapartei, a new sauropod dinosaur from La Amarga Formation (Lower Cretaceous), northwestern Patagonia, Neuquén Province, Argentina. Geobios 39: 695-707.

Salgado L, Coria RA, Calvo JO. 1997. Evolution of titanosaurid sauropods. I: phylogenetic analysis based on the postcranial evidence. Ameghiniana 34: 3-32.

Salgado L, Garrido AC, Cocca SE, Cocca JR. 2004. Lower Cretaceous rebbachisaurid sauropods from Cerro Aguada del León, Neuquén Province, northwestern Patagonia, Argentina. Journal of Vertebrate Paleontology 24: 903-912.

Salgado L, Novas FE, Suarez M, Cruz R, Isasi M, Rubilar-Rogers D, Vargas A. 2015. Late Jurassic sauropods in Chilean Patagonia. Ameghiniana 52: 418-429.

Sander PM, Mateus O, Laven T, Knötschke N. 2006. Bone histology indicates insular dwarfism in a new Late Jurassic sauropod dinosaur. Nature 441: 739-741.

Santucci RM, Arruda-Campos AC. 2011. A new sauropod (Macronaria, Titanosauria) from the Adamantina Formation, Bauru Group, Upper Cretaceous of Brazil and the phylogenetic relationships of Aeolosaurini. Zootaxa 3085: 1-33.

Santucci RM, Bertini RJ. 2006. A new titanosaur from western São Paulo State, Upper Cretaceous Bauru Group, south-east Brazil. Palaeontology 49: 59-66.

Sanz JL, Buscalioni AD, Casanovas ML, Santafé JV. 1987. Dinosaurios del Cretacico Inferior de Galve (Teruel, España). Estudios Geológicos Volumen Extraordinario, Galve-Tremp: 4564.

Sanz JL, Powell JE, Le Loueff J, Martinez R, Pereda Suberbiola X. 1999. Sauropod remains from the Upper Cretaceous of Laño (northcentral Spain). Titanosaur phylogenetic relationships. Estudios del Museo de Ciencias Naturales de Alava 14: 235-255.

Schwarz D, Fritsch G. 2006. Pneumatic structures in the cervical vertebrae of the Late Jurassic (Kimmerigian-Tithonian) Tendaguru sauropods Brachiosaurus brancai and Dicraeosaurus. Eclogae Geologicae Helvetiae 99: 65-78.

Schwarz D, Frey E, Meyer CA. 2007. Pneumaticity and soft-tissue reconstructions in the neck of diplodocid and dicraeosaurid sauropods. Acta Palaeontologica Polonica 52: 167-188. 
Schwarz-Wings D, Böhm N. 2014. A morphometric approach to the specific separation of the humeri and femora of Dicraeosaurus from the Late Jurassic of Tendaguru/Tanzania. Acta Palaeontologica Polonica 59: 81-98.

Scotese CR, Boucot AJ, McKerrow WS. 1999. Gondwanan paleogeography and paleoclimatology. Journal of African Earth Sciences 28: 99-114.

Seeley HG. 1869. Index to the fossil remains of Aves, Ornithosauria and Reptilia, from the Secondary system of strata arranged in the Woodwardian Museum of the University of Cambridge. With a prefatory notice by the Rev. Adam Sedgwick. Deighton, Bell and Co., Cambridge, 143 pp.

Sekiya T. 2011. Re-examination of Chuanjiesaurus anaensis (Dinosauria: Sauropoda) from the Middle Jurassic Chuanjie Formation, Lufeng County, Yunnan Province, southwest China. Memoir of the Fukui Prefectural Dinosaur Museum 10: 1-54.

Sellwood BW, Valdes PJ. 2008. Jurassic Climates. Proceedings of the Geologists' Association 119: 5-17.

Sereno PC, Forster CA, Rogers RR, Monetta AM. 1993. Primitive dinosaur skeleton from Argentina and the early evolution of Dinosauria. Nature 361: 64-66.

Sereno PC, Wilson JA, Larsson HCE, Dutheil DB, Sues HD. 1994. Early Cretaceous dinosaurs from the Sahara. Science 265: 267-271.

Sereno PC, Beck AL, Dutheil DB, Larsson HCE, Lyon GH, Moussa B, Sadleir RW, Sidor CA, Varricchio DJ, Wilson GP, Wilson JA. 1999. Cretaceous sauropods from the Sahara and the uneven rate of skeletal evolution among dinosaurs. Science 286: 1342-1347.

Sereno PC, Wilson JA, Witmer LM, Whitlock JA, Maga A, Ide O, Rowe TA. 2007. Structural extremes in a Cretaceous dinosaur. PLOS ONE 2: e1230.

Seton M, Müller RD, Zahirovic S, Gaina C, Torsvik T, Shepard G, Talsma A, Gurnis M, Turner M, Maus S, Chandler M. 2012. Global continental and ocean basin reconstructions since 200 Ma. Earth-Science Reviews 113: 212-270.

Smith AG, Smith DG, Funnell BM. 1994. Atlas of Mesozoic and Cenozoic Coastlines. Cambridge University Press, Cambridge, UK.

Smith JB, Lamanna MC, Lacovara KJ, Dodson P, Smith JR, Poole JC, Giegengack R, Attia Y. 2001. A giant sauropod dinosaur from an Upper Cretaceous mangrove deposit in Egypt. Science 292: 1704-1706.

Souto PRF, Fernandes MA. 2017. Paleobiogeographical significance of the Late Jurassic continental fauna from western Gondwana. Palaeoworld 26: 230-240.

Starrfelt J, Liow LH. 2016. How many dinosaur species were there? Fossil bias and true richness estimated using a Poisson sampling model. Philosophical Transactions of the Royal Society Series B 371: 20150219.

Sternfeld R. 1911. Zur Nomenklatur der Gattung Gigantosaurus Fraas. Sitzungsberichte der Gesellschaft Naturforschender Freunde zu Berlin 1911: 398.

Suñer M, Santisteban C, Galobart À. 2009. Direct evidence of Titanosauriformes found in a Upper Jurassic site of Alpuente (Los Serranos, Valencia, Spain). In: Delgado-Buscalioni A, Fregenal-Martínez M, eds. Abstracts of the Tenth International Symposium on Mesozoic Terrestrial Ecosystems and Biota, UAM Ediciones, 139-140.

Suteethorn S, Le Loeuff J, Buffetaut E, Suteethorn V. 2010. Description of topotypes of Phuwiangosaurus sirindhornae, a sauropod from the Sao Khua Formation (Early Cretaceous) of Thailand, and their phylogenetic implications. Neues Jahrbuch für Geologie und Paläontologie - Abhandlungen 256: 109-121. 
Suteethorn S, Loeuff JL, Buffetaut E, Suteethorn V, Wongko K. 2013. First evidence of a mamenchisaurid dinosaur from the Late Jurassic/Early Cretaceous Phu Kradung Formation of Thailand. Acta Palaeontologica Polonica 58: 459-469.

Taylor MP. 2009. A re-evaluation of Brachiosaurus altithorax Riggs 1903 (Dinosauria, Sauropoda) and its generic separation from Giraffatitan brancai (Janensch 1914). Journal of Vertebrate Paleontology 29: 787-806.

Taylor MP. 2018. Xenoposeidon is the earliest known rebbachisaurid sauropod dinosaur. PeerJ 6: e5212.

Taylor MP, Naish D. 2007. An unusual new neosauropod dinosaur from the Lower Cretaceous Hastings Beds Group of East Sussex, England. Palaeontology 50: 1547-1564.

Tidwell V, Carpenter K, Brooks W. 1999. New sauropod from the Lower Cretaceous of Utah, USA. Oryctos 2: 21-37.

Tidwell V, Carpenter K, Meyer S. 2001. New titanosauriform (Sauropoda) from the Poison Strip Member of the Cedar Mountain Formation (Lower Cretaceous), Utah. In: Tanke DH, Carpenter K, ed. Mesozoic Vertebrate Life. Indiana University Press, 139-165.

Torcida Fernández-Baldor F. 2012. Sistemática, filogenia y análisis paleobiogeográfico de Demandasaurus darwini (Sauropoda, Rebbachisauridae) del Barremiense SuperiorAptiense de Burgos (España). Unpublished Ph.D dissertation, Universidad Zaragoza, Spain.

Torcida Fernández-Baldor F, Canudo JI, Huerta P, Montero D, Pereda Suberbiola X, Salgado L. 2011. Demandasaurus darwini, a new rebbachisaurid sauropod from the Early Cretaceous of the Iberian Peninsula. Acta Palaeontologica Polonica 56: 535-552.

Torcida Fernández-Baldor F, Canudo JI, Huerta P, Moreno-Azanza M, Montero D. 2017. Europatitan eastwoodi, a new sauropod from the lower Cretaceous of Iberia in the initial radiation of somphospondylans in Laurasia. PeerJ 5: e3409.

Tortosa T, Buffetaut E, Vialle N, Dutour Y, Turini E, Cheylan G. 2014. A new abelisaurid dinosaur from the Late Cretaceous of southern France: Palaeobiogeographical implications. Annales de Paléontologie 100: 63-86.

Tschopp E, Mateus O. 2013. The skull and neck of a new flagellicaudatan sauropod from the Morrison Formation and its implication for the evolution and ontogeny of diplodocid dinosaurs. Journal of Systematic Palaeontology 11: 853-888.

Tschopp E, Mateus O. 2017. Osteology of Galeamopus pabsti sp. nov. (Sauropoda: Diplodocidae), with implications for neurocentral closure timing, and the cervico-dorsal transition in diplodocids. PeerJ 5: e3179.

Tschopp E, Mateus O, Benson RBJ 2015a. A specimen-level phylogenetic analysis and taxonomic revision of Diplodocidae (Dinosauria, Sauropoda). PeerJ 3: e857.

Tschopp E, Wings O, Frauenfelder T, Brinkmann, W. 2015b. Articulated bone sets of manus and pedes of Camarasaurus (Sauropoda, Dinosauria). Palaeontologia Electronica 18: 1-65.

Tsuihiji T, Watabe M, Tsogtbaatar K, Tsubamoto T, Barsbold R, Suzuki S, Lee AH, Ridgely RC, Kawahara Y, Witmer LM. 2011. Cranial osteology of juvenile spacimens of Tarbosaurus bataar (Theropoda, Tyrannosauridae) from the Nemegt Formation (Upper Cretaceous of Bugin Tzav, Mongolia). Journal of Vertebrate Paleontology 31: 497-517.

Turner AH, Smith ND, Callery JA. 2009. Gauging the effects of sampling failure in biogeographic analysis. Journal of Biogeography 36: 612-625.

Unwin DM, Heinrich W-D. 1999. On a pterosaur jaw from the Upper Jurassic of Tendaguru (Tanzania). Mitteilungen aus dem Museum für Naturkunde in Berlin, Geowissenschaftliche Reihe 2: 121-134.

Upchurch P. 1995. The evolutionary history of sauropod dinosaurs. Philosophical Transactions of the Royal Society of London, Series B 349: 365-390. 
Upchurch P. 1998. The phylogenetic relationships of sauropod dinosaurs. Zoological Journal of the Linnean Society 124: 43-103.

Upchurch P. 1999. The phylogenetic relationships of the Nemegtosauridae (Saurischia, Sauropoda). Journal of Vertebrate Paleontology 19: 106-125.

Upchurch P. 2008. Gondwanan break-up: legacies of a lost world? Trends in Ecology and Evolution 23: 229-236.

Upchurch P, Mannion PD. 2009. The first diplodocid from Asia and its implications for the evolutionary history of sauropod dinosaurs. Palaeontology 52: 1195-1207.

Upchurch P, Martin J. 2002. The Rutland Cetiosaurus: The anatomy and relationships of a Middle Jurassic British sauropod dinosaur. Palaeontology 45: 1049-1074.

Upchurch P, Martin J. 2003. The anatomy and taxonomy of Cetiosaurus (Saurischia: Sauropoda) from the Middle Jurassic of England. Journal of Vertebrate Paleontology 23: 208-231.

Upchurch P, Barrett PM, Dodson P. 2004. Sauropoda. In: Weishampel DB, Dodson P, Osmólska H, eds. The Dinosauria, 2nd edn. Berkeley: University of California Press, 259324.

Upchurch P, Barrett PM, Galton PM. 2007. The phylogenetic relationships of basal sauropodomorphs: implications for the origin of sauropods. Special Papers in Palaeontology 77: 57-90.

Upchurch P, Hunn CA, Norman DB. 2002. An analysis of dinosaurian biogeography: evidence for the existence of vicariance and dispersal patterns caused by geological events. Proceedings of the Royal Society of London, Series B 269: 613-621.

Upchurch P, Mannion PD, Barrett PM. 2011a. Sauropod dinosaurs. In: Batten DJ, ed. Field Guide to English Wealden Fossils. London: Palaeontological Association, 476-525.

Upchurch P, Mannion PD, Butler RJ, Benson RBJ, Carrano MT. 2011b. Geological and anthropogenic controls on the sampling of the terrestrial fossil record: a case study from the Dinosauria. Geological Society, London, Special Publications 358: 209-240.

Upchurch P, Mannion PD, Taylor MP. 2015. The Anatomy and Phylogenetic Relationships of "Pelorosaurus" becklesii (Neosauropoda, Macronaria) from the Early Cretaceous of England. PLOS ONE 10: e0125819.

Virchow H. 1919. Atlas und epistropheus bei den Schildkröten. Sitzungsberichte der Gesellschaft Naturforschender Freunde zu Berlin 1919: 303-332.

Volkheimer W. 1969. Palaeoclimatic Evolution in Argentina and Relations with other Regions of Gondwana. In: Amos AJ, ed. Gondwana Stratigraphy (1st IUGS Gondwana Symposium, Buenos Aires, 1967). UNESCO, Paris, 551-587.

Volkheimer W, Rauhut OWM, Quattrocchio ME, Martinez MA. 2008. Jurassic Paleoclimates in Argentina, a review. Revista de la Asociación Geológica Argentina 63: 549-556.

Wang J, Ye Y, Pei R, Tian Y, Feng C, Zheng D, Chang S-C. 2018. Age of Jurassic basal sauropods in Sichuan, China: A reappraisal of basal sauropod evolution. Geological Society of America Bulletin (doi: 10.1130/B31910.1).

Wedel MJ. 2003. The evolution of vertebral pneumaticity in sauropod dinosaurs. Journal of Vertebrate Paleontology 23: 344-357.

Wedel MJ. 2005. Postcranial skeletal pneumaticity in sauropods and its implications for mass estimates. In: Curry Rogers KA, Wilson JA, eds. The sauropods: evolution and paleobiology. Berkeley; Los Angeles: University of California Press, 201-228.

Wedel MJ, Cifelli RL, Sanders RK. 2000. Osteology, paleobiology, and relationships of the sauropod dinosaur Sauroposeidon. Acta Palaeontologica Polonica 45: 343-388. 
Weishampel DB, Barrett PM, Coria RA, Le Loeuff J, Gomani ES, Zhao Z, Xu X, Sahni A, Noto C. 2004. Dinosaur distribution. In: Weishampel DB, Dodson $\mathrm{P}$, Osmólska $\mathrm{H}$, eds. The Dinosauria, 2nd edn. Berkeley: University of California Press, 517-606.

Whiteside JH, Grogan DS, Olsen PE, Kent DV. 2011. Climatically driven biogeographic provinces of Late Triassic tropical Pangea. Proceedings of the National Academy of Sciences, USA 108: 8972-8977.

Whitlock JA. 2011a. A phylogenetic analysis of Diplodocoidea (Saurischia: Sauropoda). Zoological Journal of the Linnean Society 161: 872-915.

Whitlock JA. 2011b. Re-evaluation of Australodocus bohetii, a putative diplodocoid sauropod from the Tendaguru Formation of Tanzania, with comment on Late Jurassic sauropod faunal diversity and palaeoecology. Palaeogeography, Palaeoclimatology, Palaeoecology 309: 333-341.

Whitlock JA Harris, JD. 2010. The dentary of Suuwassea (Sauropoda: Diplodocoidea). Journal of Vertebrate Paleontology 30: 1637-1641.

Whitlock JA, D'Emic MD, Wilson JA. 2011. Cretaceous diplodocids in Asia? Re-evaluating the phylogenetic affinities of a fragmentary specimen. Palaeontology 54: 351-364.

Whitlock JA, Wilson JA, Lamanna MC. 2010. Description of a nearly complete juvenile skull of Diplodocus (Sauropoda: Diplodocoidea) from the Late Jurassic of North America. Journal of Vertebrate Paleontology 30: 442-457.

Wild R. 1991. Janenschia n. g. robusta (E. Fraas 1908) pro Tornieria robusta (E. Fraas 1908) (Reptilia, Saurischia, Sauropodomorpha). Stuttgarter Beiträge zur Naturkunde, Serie B (Geologie und Paläontologie) 173: 1-4.

Wilson JA. 1999. A nomenclature for vertebral laminae in sauropods and other saurischian dinosaurs. Journal of Vertebrate Paleontology 19: 639-653.

Wilson JA. 2002. Sauropod dinosaur phylogeny: critique and cladistic analysis. Zoological Journal of the Linnean Society 136: 217-276.

Wilson JA. 2005a. Integrating ichnofossils and body fossil records to estimate locomotor posture and spatiotemporal distribution of early sauropod dinosaurs: a stratocladistic approach. Paleobiology 31: 400-423.

Wilson JA. 2005b. Redescription of the Mongolian sauropod Nemegtosaurus mongoliensis Nowinski (Dinosauria: Saurischia) and comments on Late Cretaceous sauropod diversity. Journal of Systematic Palaeontology 3: 283-318.

Wilson JA. 2012. New vertebral laminae and patterns of serial variation in vertebral laminae of sauropod dinosaurs. Contributions from the Museum of Paleontology, University of Michigan 32: 91-110.

Wilson JA, Allain R. 2015. Osteology of Rebbachisaurus garasbae Lavocat, 1954, a diplodocoid (Dinosauria, Sauropoda) from the early Late Cretaceous-aged Kem Kem beds of southeastern Morocco. Journal of Vertebrate Paleontology 35: e1000701.

Wilson JA, Carrano MT. 1999. Titanosaurs and the origin of 'wide-gauge' trackways: a biomechanical and systematic perspective on sauropod locomotion. Paleobiology 25:252267.

Wilson JA, Sereno PC. 1998. Early evolution and higher-level phylogeny of sauropod dinosaurs. Society of Vertebrate Paleontology Memoir 5: 1-68.

Wilson JA, Upchurch P. 2003. A revision of Titanosaurus Lydekker (Dinosauria-Sauropoda), the first dinosaur genus with a 'Gondwanan' distribution. Journal of Systematic Palaeontology 1: 125-160. 
Wilson JA, Upchurch P. 2009. Redescription and reassessment of the phylogenetic affinities of Euhelopus zdanskyi (Dinosauria: Sauropoda) from the Early Cretaceous of China. Journal of Systematic Palaeontology 7: 199-239.

Wilson JA, D'Emic MD, Ikejiri, T, Moacdieh EM, Whitlock JA. 2011. A nomenclature for vertebral fossae in sauropods and other saurischian dinosaurs. PLOS ONE 6: e17114.

Wings O, Schwarz-Wings D, Fowler DW. 2011. New sauropod material from the Late Jurassic part of the Shishugou Formation (Junggar Basin, Xinjiang, NW China). Neues Jahrbuch für Geologie und Paläontologie Abhandlungen 262: 129-150.

Woodward AS. 1905. On parts of the skeleton of Cetiosaurus leedsi, a sauropodous dinosaur from the Oxford Clay of Peterborough. Proceedings of the Zoological Society of London 1905: 232-243.

Woodward HN, Lehman TM. 2009. Bone histology and microanatomy of Alamosaurus sanjuanensis (Sauropoda: Titanosauria) from the Maastrichtian of Big Bend National Park, Texas. Journal of Vertebrate Paleontology 29: 807-821.

Wu W-H, Zhou C-F, Wings O, Sekiya T, Dong Z-M. 2013. A new gigantic sauropod dinosaur from the Middle Jurassic of Shanshan, Xinjiang. Global Geology 32: 437-446.

Xing L, Miyashita T, Zhang J, Li D, Ye Y, Sekiya T, Wang F, Currie PJ 2015. A new sauropod dinosaur from the Late Jurassic of China and the diversity, distribution, and relationships of mamenchisaurids. Journal of Vertebrate Paleontology 35: e889701.

Xu J, Li Z. 2015. Middle-Late Mesozoic sedimentary provenances of the Luxi and Jiaolai areas: Implications for tectonic evolution of the North China Block. Journal of Asian Earth Sciences 111: 284-301.

Xu X, Clark JM, Mo J, Choiniere J, Forster CA, Erickson GM, Hone DWE, Sullivan C, Eberth DA, Nesbitt S, Zhao Q, Hernandez R, Jia C-K, Han F-I, Guo Y. 2009. A Jurassic ceratosaur from China helps clarify avian digital homologies. Nature 459: 940-944.

Xu X, Upchurch P, Mannion PD, Barrett PM, Regalado-Fernandez OR, Mo J, Ma J, Liu H. 2018. A new Middle Jurassic diplodocoid suggests an earlier dispersal and diversification of sauropod dinosaurs. Nature Communications 9: 2300.

Yates AM. 2007. The first complete skull of the Triassic dinosaur Melanorosaurus Haughton (Sauropodomorpha: Anchisauria). Special Papers in Palaeontology 77: 9-55.

Yates AM, Kitching J. 2003. The earliest known sauropod dinosaur and the first steps towards sauropod locomotion. Proceedings of the Royal Society of London, Series B 270: 1753-1758.

Ye Y, Gao Y-H, Jiang S. 2005. A new genus of sauropod from Zigong, Sichuan. Vertebrata PalAsiatica 43: 175-181.

You H-L, Li D-Q. 2009. The first well-preserved Early Cretaceous brachiosaurid dinosaur in Asia. Proceedings of the Royal Society of London, Series B 276: 4077-4082.

You H-L, Tang F, Luo Z. 2003. A new basal titanosaur (Dinosauria: Sauropoda) from the Early Cretaceous of China. Acta Geologica Sinica 77: 424-429.

Young CC. 1954. On a new sauropod from Yiping, Szechuan, China. Acta Paleontologica Sinica 2: 355-369.

Young CC. 1958. New sauropods from China. Vertebrata PalAsiatica 2: 1-28.

Young CC, Zhao X-J. 1972. Mamenchisaurus hochuanensis sp. nov. Institute of Vertebrate Paleontology and Paleoanthropology Monographs (Series A) 8: 1-30.

Zaher H, Pol D, Carvalho AB, Nascimento PM, Riccomini C, Larson P, Juarez-Valieri R, PiresDomingues R, Silva NJ, Campos DA. 2011. A complete skull of an Early Cretaceous sauropod and the evolution of advanced titanosaurians. PLOS ONE 6: e16663. 
Zhang Y-H. 1988. The Middle Jurassic dinosaur fauna from Dashanpu, Zigong, Sichuan. Sauropod dinosaurs. Shunosaurus. Sichuan Publishing House of Science and Technology, Chengdu, China, 106 pp.

Tables

Table 1. Measurements of the right hindlimb elements of the holotype of Janenschia robusta (SMNS 12144). All measurements are in millimetres.

\begin{tabular}{llc}
\hline Element & Dimension & Measurement \\
\hline Femur & Mediolateral width of shaft & 244 \\
& Anteroposterior width of shaft & 163 \\
& Mediolateral width of distal end & 385 \\
& Anteroposterior width of distal end (measured on tibial condyle) & 315 \\
Tibia & Anteroposterior width of distal end (measured on fibular condyle) & 288 \\
& Proximodistal length & 823 \\
& Mediolateral width of proximal end & 307 \\
& Anteroposterior width of proximal end & 275 \\
& Length of lateral projection of cnemial crest & 60 \\
& Long axis diameter at midshaft & 140 \\
& Diameter perpendicular to long axis diameter at midshaft & 87 \\
& Minimum shaft circumference & 397 \\
& Mediolateral width of distal end & 265 \\
& Anteroposterior width of distal end (measured on medial malleolus) & 204 \\
& Anteroposterior width of distal end (measured on lateral malleolus) & 145 \\
Fibula & Proximodistal length & 845 \\
& Mediolateral width of proximal end & 74 \\
& Anteroposterior width of proximal end & 204 \\
& Mediolateral width at midshaft & 63 \\
& Anteroposterior width at midshaft & 97 \\
& Minimum shaft circumference & 260 \\
& Mediolateral width of distal end & 108 \\
& Anteroposterior width of distal end & 130 \\
& Maximum mediolateral width & 276 \\
& Anteroposterior width (measured on posterior expansion) & 157 \\
& Anteroposterior width (measured on lateral margin) & 134 \\
& Maximum proximodistal height & 129 \\
\hline
\end{tabular}

Table 2. Measurements of the right metatarsals of the holotype of Janenschia robusta (SMNS 12144). All measurements are in millimetres.

\begin{tabular}{l|ccccc}
\hline Dimension & Mt.I & Mt.II & Mt.III & Mt.IV & Mt.V \\
\hline Maximum proximodistal length & 143 & 161 & 167 & 153 & 107 \\
Proximal end dorsoventral height & 133 & 116 & 95 & 104 & 66 \\
Proximal end mediolateral width & 117 & 103 & 81 & 71 & 125 \\
Midshaft dorsoventral height & 73 & 61 & 48 & 49 & 41 \\
Midshaft mediolateral width & 105 & 62 & 42 & 35 & 66 \\
Distal end dorsoventral height & 82 & 83 & 65 & 53 & 54 \\
\hline
\end{tabular}




\begin{tabular}{l|lllll}
\hline Distal end mediolateral width & 127 & 109 & 95 & 76 & 77 \\
\hline
\end{tabular}

Table 3. Measurements of the right pedal phalanges of the holotype of Janenschia robusta (SMNS 12144). An asterisk $\left({ }^{*}\right)$ denotes a measurement based on an incomplete element. 'Length as restored' reflects the restoration of the distally incomplete unguals - these measurements represent estimates of the lengths of the complete elements. All measurements are in millimetres.

\begin{tabular}{|c|c|c|c|c|c|c|c|c|c|c|c|}
\hline Dimension & $\mathrm{I}-1$ & $\mathrm{I}-2$ & II-1 & II-2 & II-3 & III-1 & III-2 & III-3 & IV-1 & IV-2 & V-1 \\
\hline Maximum proximodistal length & 68 & $200 *$ & 76 & 28 & $138^{*}$ & 59 & 28 & $104^{*}$ & 57 & 49 & 32 \\
\hline Proximal end dorsoventral height & 75 & 120 & 78 & 59 & 85 & 55 & 42 & 58 & - & 35 & 34 \\
\hline Proximal end mediolateral width & 87 & 65 & 85 & 76 & 54 & 66 & 56 & 45 & 58 & 62 & 55 \\
\hline Distal end dorsoventral height & 84 & - & 88 & - & - & - & - & - & 45 & - & - \\
\hline Distal end mediolateral width & 79 & - & 85 & - & - & - & - & - & 65 & - & - \\
\hline Length as restored & - & 255 & - & - & 181 & - & - & 122 & - & - & - \\
\hline
\end{tabular}

Table 4. Measurements of topotypic elements of the holotype of Janenschia robusta, from Quarry B (MB.R.2090). An asterisk $\left({ }^{*}\right)$ denotes a measurement based on an incomplete element. All measurements are in millimetres.

\begin{tabular}{llc}
\hline Element & Dimension & Measurement \\
\hline Left pubis (B8) & Length & 939 \\
& Anteroposterior length of iliac articular surface & 293 \\
& Maximum mediolateral width of iliac articular surface & 173 \\
& Approximate dorsoventral height of ischiadic articulation & $\sim 415^{*}$ \\
& Anteroposterior length of distal end & 327 \\
& Maximum mediolateral width of distal end & 221 \\
Right ischium (B11) & Preserved length & $965^{*}$ \\
Right ischium (B13) & Length & 903 \\
& Maximum anteroposterior length of iliac peduncle & 140 \\
& Maximum mediolateral width of iliac peduncle & 150 \\
& Anteroposterior length of acetabulum & 205 \\
& Anteroposterior length of proximal plate & 293 \\
& Maximum dorsoventral height of distal blade & 240 \\
& Maximum mediolateral width of distal blade & 90 \\
& Preserved length (distal half only) & $420^{*}$ \\
& Long axis dimension of shaft & 165 \\
& Dimension of shaft perpendicular to long axis & 87 \\
& Minimum shaft circumference (as preserved) & 412 \\
& Distal end mediolateral width & $255^{*}$ \\
& Distal end maximum anteroposterior length & $177^{*}$ \\
\hline
\end{tabular}

Table 5. Measurements of referred forelimb material of Janenschia robusta from Quarry P. An asterisk $\left({ }^{*}\right)$ denotes a measurement based on an incomplete element. All measurements are in millimetres. 
Left humerus (P8)

Length

905

Proximal end maximum mediolateral width

395

Distance from proximal end to distal tip of dtp crest 430

Minimum shaft circumference

425

Mediolateral width at midshaft

148

Anteroposterior width at midshaft

$\sim 100$

Distal end maximum mediolateral width

330

Distal end maximum anteroposterior width 194

Left radius ( $P 10)$

Length

606

Left radius $(P 11)$

Length as preserved

$605^{*}$

Proximal end mediolateral width

178

Proximal end anteroposterior width 163

Distal end mediolateral width 203

Distal end anteroposterior width 130

Left ulna (P9)

Length

676

Proximal end mediolateral width

318

Proximal end anteroposterior width

195*

Distal end mediolateral width

107

Distal end anteroposterior length

176

Length

659

Proximal end mediolateral width

316

Proximal end anteroposterior length

267*

Distal end mediolateral width

Distal end anteroposterior length

204

Maximum mediolateral width

159

Maximum proximodistal height

73

Maximum anteroposterior length

105

Left manual ungual Length

(P32)

Proximal end dorsoventral height

92

Proximal end mediolateral width

70

Manual phalanx Maximum proximodistal length

50

(P54)

Maximum dorsoventral height

Maximum mediolateral width

Table 6. Measurements of referred hindlimb material of Janenschia robusta from Quarry P. An asterisk $\left({ }^{*}\right)$ denotes a measurement based on an incomplete element. All measurements are in millimetres.

\begin{tabular}{llcc}
\hline Element & Dimension & Measurement \\
\hline Left femur (P22) & Length & $\sim 1249$ \\
& Distance from proximal end to proximal tip of $4^{\text {th }}$ & 480 \\
& trochanter & & \\
& Proximodistal length of $4^{\text {th }}$ trochanter & & \\
& Minimum shaft circumference & 566 \\
& Mediolateral width at midshaft & 222 \\
& Anteroposterior width at midshaft & 129 \\
\hline
\end{tabular}


Maximum mediolateral width of distal end

Anteroposterior length of distal end (tibial condyle)

Anteroposterior length of distal end (fibular condyle)

265

Left tibia (P2)

Proximodistal length

790

Mediolateral width of proximal end

275

Anteroposterior width of proximal end

256

Mediolateral width of distal end

296

Maximum anteroposterior width of distal end

$186^{*}$

Proximodistal length

846

Mediolateral width of proximal end

289

Anteroposterior width of proximal end

283

Long axis dimension of shaft

158

Dimension of shaft perpendicular to long axis

87

Mediolateral width of distal end

310

Maximum anteroposterior width of distal end 181*

Left fibula (P3)

Length

$\sim 880$

Proximal end anteroposterior length

182

Proximal end maximum mediolateral width

73

Distal end anteroposterior length

138

Distal end maximum mediolateral width

105

Length as preserved

$627^{*}$

Proximal end anteroposterior length

193

Proximal end maximum mediolateral width 78

Left astragalus (P4)

Maximum mediolateral width

260

103

Maximum proximodistal height

165

Maximum anteroposterior length

302

Maximum mediolateral width

127

Maximum proximodistal height

175

Table 7. Measurements of the holotypic dorsal vertebrae (MB.R.2092.1-2) and tentatively referred cervical vertebra (MB.R.2091.31) of Tendaguria tanzaniensis. Neural arch height is measured from the dorsal margin of the centrum up to the base of the postzygapophyses. Neural spine height is measured from the base of the postzygapophyses up to the neural spine summit. All measurements are in millimetres.

\begin{tabular}{l|c|c|c}
\hline Dimension & 2092.2 (Dv2) & 2092.1 (Dv3) & $\mathbf{2 0 9 1 . 3 1}$ \\
\hline Centrum length (excluding ball) & 175 & 200 & 242 \\
Centrum length (including ball) & 244 & 250 & 288 \\
Posterior centrum height & 249 & 228 & 142 \\
Posterior centrum width & 378 & 351 & 181 \\
Neural arch height & 116 & 112 & 70 \\
Total mediolateral width across prezygapophyses & 494 & 448 & - \\
Neural spine height & 170 & 162 & 76 \\
Maximum mediolateral width of neural spine & 61 & - & 71 \\
Maximum anteroposterior length of neural spine & 34 & 34 & 72 \\
Total width across diapophyses & 1020 & 1020 & - \\
Total vertebra height & 494 & 496 & 288 \\
\hline
\end{tabular}


Table 8. Ratios of mediolateral width to dorsoventral height of posterior articular surfaces of anteriormost dorsal centra (Dv1-3) for an array of eusauropod taxa, arranged in order of increasing values.

\begin{tabular}{l|c}
\hline Taxon & Ratio \\
\hline Jobaria tiguidensis (MNN F249: PDM pers. obs. 2012) & 0.75 \\
Euhelopus zdanskyi (Wilson \& Upchurch, 2009) & 0.90 \\
Galveosaurus herreroi (Barco, Canudo \& Cuenca-Bescós, 2006) & 0.91 \\
Nigersaurus taqueti (MNN unnumbered: PDM pers. obs. 2010) & 0.95 \\
Comahuesaurus windhauseni (Carballido et al., 2012) & 1.00 \\
Dicraeosaurus hansemanni (Janensch, 1929b) & 1.05 \\
Europasaurus holgeri (Carballido \& Sander, 2014) & 1.07 \\
Haplocanthosaurus priscus (Hatcher, 1903) & 1.09 \\
Diplodocus carnegii (Hatcher, 1901) & 1.11 \\
Patagosaurus fariasi (PVL 4170: PDM \& PU pers. obs. 2013) & 1.14 \\
Sauroposeidon proteles (Rose, 2007) & 1.15 \\
Mamenchisaurus youngi (Ouyang \& Ye, 2002) & 1.18 \\
Bellusaurus sui (Mo, 2013) & 1.19 \\
Camarasaurus supremus (Osborn \& Mook, 1921) & 1.20 \\
Omeisaurus tianfuensis (He et al., 1988) & 1.23 \\
Amargasaurus cazaui (MACN N-15: PDM \& PU pers. obs. 2013) & 1.23 \\
Brachytrachelopan mesai (MPEF-PV 1716: PDM \& PU pers. obs. 2013) & 1.30 \\
Moabosaurus utahensis (Britt et al., 2017) & 1.35 \\
Puertasaurus reuili (Novas et al., 2005) & 1.36 \\
Lourinhasaurus alenquerensis (Mocho et al., 2014) & 1.37 \\
Apatosaurus louisae (Gilmore, 1936) & 1.40 \\
Mendozasaurus neguyelap (González Riga, 2003) & 1.40 \\
Malawisaurus dixeyi (Gomani, 2005) & 1.45 \\
Rapetosaurus krausei (Curry Rogers, 2009) & 1.47 \\
Opisthocoelicaudia skarzynskii (Borsuk-Białynicka, 1977) & 1.48 \\
Tendaguria tanzaniensis (this study) & 1.53 \\
Mierasaurus bobyongi (Royo-Torres et al., 2017a) & 1.80 \\
Ligabuesaurus leanzai (Bonaparte, González Riga \& Apesteguía, 2006) & 2.22 \\
\hline & \\
\hline
\end{tabular}

Table 9. Measurements of the caudal vertebrae of the holotype and referred individual of Wamweracaudia keranjei (MB.R.2091.1-30, 3817.1, 3817.2 and 2094). Note that the caudal vertebrae are numbered inversely, such that MB.R.2091.30 is the most anterior preserved element and MB.R.2091.1 is the most posterior. Abbreviations: Cd, caudal vertebra; CL, centrum length (excluding ball); CLB, centrum length (including ball); $\mathrm{ACH}$, anterior centrum dorsoventral height; $\mathrm{ACW}$, anterior centrum mediolateral width; $\mathrm{PCH}$, posterior centrum dorsoventral height; PCW, posterior centrum mediolateral width; DFA, distance from anterior end of centrum to anterior margin of neural arch; DFP, distance from posterior end of centrum to posterior margin of neural arch; $\mathrm{NAH}$, neural arch dorsoventral height (measured from dorsal margin of centrum up to the base of the postzygapophyses); NSH, neural spine dorsoventral height (measured from base of postzygapophyses up to neural spine summit); NSL, neural spine maximum anteroposterior length (measured above SPOLs); NSW, neural spine maximum mediolateral width; CRW, caudal rib width (measured from 
distal tip of rib to midline of vertebra); $\mathrm{CCl}$, condylar convexity index (anteroposterior length of posterior condylar ball divided by mean radius of the condyle [i.e. mediolateral width + dorsoventral height of articular surface, divided by 4]; Whitlock et al., 2011; Mannion et al., 2013). A hash sign (\#) denotes a measurement based only on the right half of the centrum, and an asterisk $\left({ }^{*}\right)$ denotes a measurement based on an incomplete element. All measurements are in millimetres.

\begin{tabular}{|c|c|c|c|c|c|c|c|c|c|c|c|c|c|c|}
\hline $\mathrm{Cd}$ & $\mathrm{CL}$ & CLB & $\mathrm{ACH}$ & ACW & $\mathrm{PCH}$ & PCW & DFA & DFP & NAH & NSH & NSL & NSW & CRW & $\mathrm{CCl}$ \\
\hline 30 & - & - & - & - & - & - & - & - & - & 245 & 68 & 80 & 235 & - \\
\hline 29 & $\sim 105$ & - & - & - & - & - & - & - & 80 & 241 & 61 & 67 & 205 & $\sim 0.62$ \\
\hline 28 & 119 & 174 & - & - & - & - & - & - & 83 & 210 & 65 & 64 & 202 & $\sim 0.57$ \\
\hline 27 & 131 & 176 & 167 & - & - & - & - & - & 77 & 199 & 70 & 60 & 187 & $\sim 0.37$ \\
\hline 26 & 130 & 157 & 158 & 82\# & - & - & - & - & 65 & 196 & 64 & 58 & 180 & 0.34 \\
\hline 25 & - & - & - & $90 \#$ & - & - & - & - & - & - & - & - & 160 & - \\
\hline 24 & 117 & 136 & 132 & $78 \#$ & - & - & - & - & 66 & 155 & 50 & 62 & - & 0.26 \\
\hline 23 & 117 & - & 133 & 79\# & - & - & - & - & 50 & 146 & 57 & 72 & - & - \\
\hline 22 & 117 & - & 147 & 131 & 135 & 126 & - & - & 52 & 143 & 51 & 51 & - & - \\
\hline 21 & 125 & - & 145 & 139 & 133 & 131 & - & - & 47 & 140 & 55 & 55 & - & - \\
\hline 20 & 125 & - & $\sim 143$ & 150 & 131 & 142 & - & - & - & - & 53 & 54 & - & - \\
\hline 19 & 124 & - & 143 & 150 & 135 & 140 & - & - & - & - & 57 & 49 & - & - \\
\hline 18 & 125 & - & 136 & 144 & 130 & 126 & - & - & 30 & 118 & 65 & 51 & - & - \\
\hline 17 & 129 & - & 136 & 136 & 130 & 124 & - & - & - & - & 73 & 45 & - & - \\
\hline 16 & 130 & - & 128 & 123 & 128 & 116 & 20 & 26 & 36 & 111 & 78 & 38 & - & - \\
\hline 15 & 137 & - & 129 & 119 & 121 & 110 & 21 & 26 & 44 & 92 & 87 & 39 & - & - \\
\hline 14 & 138 & - & 122 & 113 & 122 & 106 & 22 & 27 & 30 & 102 & 102 & 37 & - & - \\
\hline 13 & 140 & - & 128 & 110 & 120 & 109 & 20 & 25 & 29 & 94 & 110 & 38 & - & - \\
\hline 12 & 143 & - & 121 & 106 & 112 & 90 & 20 & 26 & 32 & 83 & 112 & 31 & - & - \\
\hline 11 & 144 & - & 115 & 95 & 110 & 94 & 21 & 26 & 32 & 84 & 118 & 29 & - & - \\
\hline 10 & 140 & - & 110 & 90 & 98 & 81 & 21 & 26 & 30 & 89 & 104 & - & - & - \\
\hline 9 & 131 & - & 100 & 85 & 95 & 76 & 16 & 23 & - & - & 100 & 25 & - & - \\
\hline 8 & 129 & - & 98 & 81 & 90 & 71 & 18 & 21 & 33 & 77 & 116 & 26 & - & - \\
\hline 7 & 126 & - & 90 & 76 & 84 & 68 & 17 & 21 & 33 & 72 & 116 & 26 & - & - \\
\hline 6 & 122 & - & 80 & 71 & 77 & 61 & 16 & 20 & 30 & 73 & 112 & 28 & - & - \\
\hline 5 & 118 & - & 74 & 61 & 73 & 57 & 14 & 19 & 32 & 70 & 102 & 28 & - & - \\
\hline 4 & 114 & - & 68 & 57 & 67 & 52 & 18 & 21 & 26 & 66 & 96 & 23 & - & - \\
\hline 3 & 110 & - & 60 & 48 & 64 & 56 & 21 & 19 & 22 & 63 & 85 & 23 & - & - \\
\hline 2 & 100 & - & 56 & 48 & 54 & 42 & 15 & 17 & 23 & 66 & 80 & 16 & - & - \\
\hline 1 & 95 & - & 49 & 45 & 52 & 43 & 17 & 18 & 25 & 63 & 75 & 20 & - & - \\
\hline 3817.1 & - & - & - & - & - & - & - & - & - & $143^{*}$ & 54 & 60 & - & - \\
\hline 3817.2 & - & - & - & - & - & - & - & - & - & $130 *$ & 50 & 70 & - & - \\
\hline 2094 & 151 & 210 & 234 & 240 & - & - & - & - & 115 & 196 & 79 & 71 & 240 & 0.50 \\
\hline
\end{tabular}

Table 10. Condylar convexity index (CCl) values for a range of eusauropod taxa with procoelous anterior caudal vertebrae, arranged in order of increasing values. For each taxon, the highest known $\mathrm{CCl}$ value is provided. $\mathrm{CCl}$ values without a reference are reproduced from the supplementary materials in Mannion et al. (2013). Non-somphospondylan taxa are emboldened. 


\begin{tabular}{l|c}
\hline Taxon & Ratio \\
\hline Tastavinsaurus sanzi & 0.13 \\
Tornieria africana (Remes, 2006) & $\mathbf{0 . 1 4}$ \\
Suuwassea emilieae (Harris, 2006a) & $\mathbf{0 . 1 8}$ \\
Apatosaurus louisae & $\mathbf{0 . 2 6}$ \\
Andesaurus delgadoi & 0.28 \\
Diplodocus carnegii & $\mathbf{0 . 3 0}$ \\
Turiasaurus riodevensis (Royo-Torres et al., 2009) & $\mathbf{0 . 3 4}$ \\
Bellusaurus sui (IVPP unnumbered: PDM \& PU pers. obs. 2007) & $\mathbf{0 . 3 7}$ \\
Paralititan stromeri (Smith et al., 2001) & 0.42 \\
Chuanjiesaurus anaensis (Sekiya, 2011) & $\mathbf{0 . 4 2}$ \\
Losillasaurus giganteus & $\mathbf{0 . 4 3}$ \\
Dicraeosaurus hansemanni (Janensch, 1929b) & $\mathbf{0 . 4 9}$ \\
Trigonosaurus pricei (Campos et al., 2005) & 0.50 \\
Elaltitan lilloi (Mannion \& Otero, 2012) & 0.55 \\
Aeolosaurus rionegrinus (Powell, 2003) & 0.61 \\
Mendozasaurus neguyelap (González Riga et al., 2018) & 0.61 \\
Wamweracaudia keranjei (this study) & $\mathbf{0 . 6 2}$ \\
Bonatitan reigi (MACN 821: PDM \& PU pers. obs. 2013) & 0.64 \\
Moabosaurus utahensis (Britt et al., 2017) & $\mathbf{0 . 6 5}$ \\
Xianshanosaurus shijiagouensis & 0.67 \\
Rapetosaurus krausei & 0.72 \\
Epachthosaurus sciuttoi (UNPSJB-PV 920: PDM \& PU pers. obs. 2013) & 0.72 \\
Mamenchisaurus hochuanensis (Young \& Zhao, 1972) & $\mathbf{0 . 7 4}$ \\
Mamenchisaurus youngi (Ouyang \& Ye, 2002) & $\mathbf{0 . 7 5}$ \\
Neuquensaurus australis (Salgado, Apesteguía \& Heredia, 2005) & 0.77 \\
Adamantisaurus mezzalirai (Santucci \& Bertini, 2006) & 0.78 \\
Saltasaurus loricatus & 0.80 \\
Dongbeititan dongi & 0.81 \\
Malawisaurus dixeyi & 0.84 \\
Baurutitan britoi (Kellner et al., 2005) & 0.88 \\
Futalognkosaurus dukei (MUCPv-323: PDM pers. obs. 2009) & 0.90 \\
Alamosaurus sanjuanensis & 0.96 \\
Daxiatitan binglingi & 1.05 \\
Lirainosaurus astibiae (Sanz et al., 1999) & 1.05 \\
\hline
\end{tabular}

Table 11. Measurements of the chevrons of the holotype of Wamweracaudia keranjei (MB.R.2091.1-30). Note that the chevrons are distally incomplete. All measurements are in millimetres.

\begin{tabular}{l|c|c}
\hline Dimension & G28 & G27 \\
\hline Total preserved dorsoventral height & 147 & 127 \\
Mediolateral width of proximal end & 83 & 83 \\
Maximum anteroposterior width of proximal end & 31 & 32 \\
Dorsoventral height of haemal canal & 60 & 34 \\
Maximum mediolateral width of haemal canal & 25 & 25 \\
Maximum mediolateral width of distal blade & 31 & 32 \\
Maximum preserved anteroposterior width of distal blade & 52 & 47 \\
\hline
\end{tabular}


Table 12. Measurements of the metacarpals of MB.R.2093 from Site Nr. 5 previously referred to Janenschia robusta. An asterisk $\left({ }^{*}\right)$ denotes a measurement based on an incomplete element. All measurements are in millimetres.

\begin{tabular}{l|ccccc}
\hline Dimension & Mc.I & Mc.II & Mc.III & Mc.IV & Mc.V \\
\hline Maximum proximodistal length & 243 & 279 & $272^{*}$ & 248 & 239 \\
Proximal end dorsoventral height & 132 & 114 & 103 & 110 & 118 \\
Proximal end mediolateral width & 93 & 98 & 84 & 80 & 74 \\
Midshaft circumference & 226 & 195 & 169 & 148 & 199 \\
Midshaft dorsoventral height & 66 & 64 & 45 & 50 & 65 \\
Midshaft mediolateral width & 69 & 71 & 50 & 45 & 68 \\
Distal end dorsoventral height & 97 & 80 & 68 & 74 & 85 \\
Distal end mediolateral width & 107 & 102 & 82 & 90 & 116 \\
\hline
\end{tabular}

Table 13. Measurements of the manual phalanges of MB.R.2093 from Site Nr. 5 previously referred to Janenschia robusta. All measurements are in millimetres.

\begin{tabular}{l|ccccccc}
\hline Dimension & I-1 & II-1 & III-1 & IV-1 & V-1 & I-2 & II-2 \\
\hline Maximum proximodistal length & 58 & 49 & 64 & 40 & 45 & 160 & 20 \\
Maximum dorsoventral height & 78 & 69 & 50 & 39 & 59 & 106 & 30 \\
Maximum mediolateral width & 90 & 72 & 78 & 69 & 84 & 69 & 43 \\
\hline
\end{tabular}

Table 14. Measurements of right ischium (MB.R. 2091.32) from Quarry G previously referred to Janenschia robusta. All measurements are in millimetres.

\begin{tabular}{lc}
\hline Dimension & Measurement \\
\hline Preserved length & $680^{*}$ \\
Anteroposterior length of iliac peduncle & 135 \\
Maximum mediolateral width of iliac peduncle & 52 \\
Dorsoventral height of pubic articulation & 205 \\
Anteroposterior length of proximal plate & 183 \\
Minimum dorsoventral height of ischial blade & 111 \\
Maximum dorsoventral height of ischial blade (at distal end) & 208 \\
Maximum mediolateral width of ischial blade (at distal end) & 95 \\
\hline
\end{tabular}

Table 15. Measurements of material from Quarry IX previously referred to Janenschia robusta. All measurements are in millimetres.

\begin{tabular}{llc}
\hline Element & Dimension & Measurement \\
\hline Right radius (v3) & Length & 491 \\
& Proximal end mediolateral width & 140 \\
& Proximal end maximum anteroposterior width & 100 \\
& Midshaft mediolateral width & 62 \\
& Midshaft anteroposterior width & 61 \\
& Distal end mediolateral width & 120 \\
& Distal end maximum anteroposterior width & 70 \\
\hline
\end{tabular}




\begin{tabular}{llc}
\hline Right femur (c1) & Length & 1320 \\
& Distance from proximal end to proximal tip of $4^{\text {th }}$ trochanter & 480 \\
& Proximodistal length of $4^{\text {th }}$ trochanter & $155^{*}$ \\
Minimum shaft circumference & 605 \\
Mediolateral width at midshaft & 244 \\
Anteroposterior width at midshaft & 135 \\
Maximum mediolateral width of distal end & 448 \\
Anteroposterior length of distal end (tibial condyle) & 345 \\
Anteroposterior length of distal end (fibular condyle) & 286 \\
\hline
\end{tabular}

Table 16. Summary of results and statistical comparisons between the six biogeographic models applied in the BioGeoBEARS analyses for the equal weights agreement subtree. The 'Ratio' in the AIC analyses is the ratio of the AIC weight for the $+\mathrm{J}$ version of the same model (e.g. DEC+J/DEC). An asterisk $\left({ }^{*}\right)$ marks those models that are regarded as best fitting the data in each analysis (see supplementary text for details).

\begin{tabular}{|c|c|c|c|c|c|c|c|}
\hline Analysis & Model & LnL & Ln likeliho & od ratio & & C analys & \\
\hline \multirow[t]{2}{*}{ Relaxed } & DEC & -335.4 & D statistic & $p$-value & $\begin{array}{l}\text { AIC } \\
674.7\end{array}$ & $\begin{array}{l}\mathrm{AIC}_{\mathrm{wt}} \\
1.3 \mathrm{e}^{-12}\end{array}$ & Ratio \\
\hline & $\mathrm{DEC}+\mathrm{J}$ & -307.0 & 56.67 & $5.2 e^{-14}$ & 620.0 & 1.00 & $7.44 e^{11}$ \\
\hline \multirow{10}{*}{ Harsh } & DIVALIKE & -370.5 & & & 744.9 & $9.8 e^{-19}$ & \\
\hline & DIVALIKE+J & -328.0 & 84.93 & $3.1 e^{-20}$ & 662.0 & 1.00 & $1.0 e^{18}$ \\
\hline & BAYAREALIKE & -290.8 & & & 585.6 & $1.3 e^{-16}$ & \\
\hline & *BAYAREALIKE+J & -253.2 & 75.16 & $4.3 e^{-18}$ & 512.4 & 1.00 & $7.7 e^{15}$ \\
\hline & DEC & -351.9 & \multirow{3}{*}{42.34} & \multirow{3}{*}{$7.7 e^{-11}$} & 707.7 & $1.7 e^{-9}$ & \multirow{3}{*}{$5.74 e^{7}$} \\
\hline & $\mathrm{DEC}+\mathrm{J}$ & -330.7 & & & 667.4 & 1.00 & \\
\hline & DIVALIKE & -404.8 & & & 813.6 & $4.1 e^{-14}$ & \\
\hline & DIVALIKE+J & -373 & \multirow[t]{2}{*}{63.64} & $1.5 e^{-15}$ & 752.0 & 1.00 & \multirow[t]{2}{*}{$2.43 e^{13}$} \\
\hline & BAYAREALIKE & -306.1 & & & 616.2 & $2.4 e^{-8}$ & \\
\hline & *BAYAREALIKE+J & -287.6 & 37.07 & $1.1 e^{-9}$ & 581.2 & 1.00 & $4.12 e^{8}$ \\
\hline
\end{tabular}

Table 17. Summary of results and statistical comparisons between the six biogeographic models applied in the BioGeoBEARS analyses for the extended implied weights agreement subtree.

\begin{tabular}{llllcccc}
\hline Analysis & Model & LnL & \multicolumn{2}{c}{ Ln likelihood ratio } & \multicolumn{3}{c}{ AIC analysis } \\
\hline \multirow{3}{*}{ Relaxed } & & & D statistic & $\boldsymbol{p}$-value & AIC & AIC $_{\text {wt }}$ & Ratio \\
& DEC & -337.9 & & & 679.6 & $9.6 \mathrm{e}^{-6}$ & \\
& DEC+J & -325.3 & 25.11 & $5.4 \mathrm{e}^{-7}$ & 656.5 & 1.00 & $1.94 \mathrm{e}^{5}$ \\
& DIVALIKE & -369.2 & & & 742.4 & $4.3 \mathrm{e}^{-7}$ & \\
& DIVALIKE+J & -353.6 & 31.32 & $2.2 \mathrm{e}^{-8}$ & 713.1 & 1.00 & $2.33 \mathrm{e}^{6}$ \\
& BAYAREALIKE & -293.1 & & & 590.2 & $2.8 \mathrm{e}^{-9}$ & \\
& *BYYREALIKE+J & -272.4 & 41.41 & $1.2 \mathrm{e}^{-10}$ & 550.8 & 1.00 & $3.60 \mathrm{e}^{8}$ \\
& & & & & & & \\
\multirow{5}{*}{ Harsh } & DEC & -353.5 & & & 711.0 & 0.0018 & \\
& DEC+J & -346.2 & 14.60 & 0.0001 & 698.4 & 1.00 & 545.2 \\
& DIVALIKE & -404.5 & & & 813.0 & $2.4 \mathrm{e}^{-9}$ & \\
\hline
\end{tabular}


Table 18. Constraint tests of alternative phylogenetic placements of enigmatic Tendaguru taxa. The phylogenetic position notes the placement of the constrained taxa relative to the original analysis (i.e. 'Janenschia position' corresponds to the position of Janesnchia in the unconstrained tree).

\begin{tabular}{|c|c|c|c|c|c|}
\hline \multirow[t]{2}{*}{ Constraint } & \multicolumn{2}{|c|}{ EWP } & \multicolumn{2}{|c|}{ EIW } & \multirow[t]{2}{*}{ Phylogenetic position } \\
\hline & MPTs & Steps & MPTs & Steps & \\
\hline No constraint & 22,704 & 2551 & 27 & 237 & $\mathrm{~N} / \mathrm{A}$ \\
\hline Janenschia + Tendaguria & 7568 & 2553 & 27 & 237.3 & Tendaguria \\
\hline Janenschia + Wamweracaudia & 742,048 & 2555 & 27 & 237 & Janenschia \\
\hline Tendaguria + Wamweracaudia & 22,704 & 2556 & 27 & 237.3 & Wamweracaudia \\
\hline $\begin{array}{l}\text { Janenschia + Tendaguria + } \\
\text { Wamweracaudia }\end{array}$ & 198,352 & 2561 & 27 & 237.5 & Janenschia \\
\hline
\end{tabular}

Figures

Figure 1. Map of the Tendaguru region, showing its location within Tanzania (inset) and the Tendaguru Formation localities yielding sauropod remains discussed in the text $(B, P, N B, G$, Oa, IX, dd). Solid lines represent rivers, and dashed lines are paths/roads through the area. Maps drawn based on information in: Janensch (1925b), Bonaparte et al. (2000), Aberhan et al. (2002), Remes (2006, 2009), and Bussert et al. (2009) [intended for double column width].

Figure 2. Janenschia robusta holotype distal right femur (SMNS 12144) in (A) proximal, (B) distal, (C) anterior, (D) medial, (E) lateral, and (F) posterior views. Abbreviations: dac, distal anterior concavity; fc, fibular condyle; fcg, fibular condylar groove; tc, tibial condyle. Scale bar $=100 \mathrm{~mm}$ [intended for double column width].

Figure 3. Janenschia robusta holotype right tibia (SMNS 12144) in (A) proximal, (B) anterior, (C) medial, (D) posterior, (E) lateral, and (F) distal views. Abbreviations: cc, cnemial crest; Im, lateral malleolus; $\mathrm{mm}$, medial malleolus; scc, second cnemial crest; tf, tuberculum fibularis. Scale bar $=200 \mathrm{~mm}$ [intended for double column width].

Figure 4. Janenschia robusta holotype right fibula (SMNS 12144) in (A) anterior, (B) medial, (C) posterior, (D) lateral, (E) proximal (anterior margin at top), and (F) distal (anterior margin at top) views. Abbreviations: Itr, lateral trochanter; sa, striated area; $\mathrm{tt}$, tuberculum tibialis. Scale bar $=200 \mathrm{~mm}$ [intended for double column width].

Figure 5. Janenschia robusta holotype right astragalus (SMNS 12144) in (A) in articulation with the the distal ends of the tibia and fibula in anterior view, (B) anterior, (C) medial, (D) posterior, (E) dorsal/proximal, (F) lateral, and (G) ventral/distal views. Abbreviations: ap, ascending process; $\mathrm{df}$, dorsal fossa; If, lateral fossa; pp, posterior process. Scale bar $=100$ $\mathrm{mm}$ [intended for double column width]. 
Figure 6. Janenschia robusta holotype right metatarsals I-V (SMNS 12144) in (A) proximal, (B) dorsal, (C) medial, (D) ventral, (E) lateral, and (F) distal views. Dorsal margin at top in parts $A$ and F; proximal end at top in parts B-E. Abbreviation: vlp, ventrolateral projection. Scale bar $=100 \mathrm{~mm}$ [intended for double column width].

Figure 7. Janenschia robusta holotype right pedal phalanges (SMNS 12144). Phalanx I-1 in (A) proximal, (B) dorsal, (C) ventral, and (D) distal views; phalanx I-2 in (E) medial, (F) proximal), and (G) lateral views; phalanx II-1 in (H) proximal, (I) dorsal, (J) ventral, and (K) distal views; phalanx II-2 in (L) proximal, (M) dorsal, and (N) distal views; phalanx II-3 in (O) medial, (P) proximal, and $(\mathrm{Q})$ lateral views; phalanx III-1 in (R) proximal, $(\mathrm{S})$ dorsal, $(\mathrm{T})$ ventral, and $(\mathrm{U})$ distal views; phalanx III-2 in (V) proximal, (W) dorsal, (X) ventral, and (Y) distal views; phalanx III-3 in (Z) medial, (AA) proximal, and (AB) lateral views; phalanx IV-1 in (AC) proximal, (AD) dorsal, $(A E)$, ventral, and (AF) distal views; phalanx IV-2 in (AG) proximal, (AH) dorsal, (AI) ventral, and (AJ) distal views; and phalanx V-1 in (AK) proximal, (AL) dorsal, (AM) ventral, and (AN) dorsal views. Scale bar $=100 \mathrm{~mm}$ [intended for double column width].

Figure 8. Janenschia robusta holotype right pes (SMNS 12144) in approximate articulation in dorsal view. Scale bar $=100 \mathrm{~mm}$ [intended for double column width].

Figure 9. Janenschia robusta topotype pelvic girdle elements: left pubis (MB.R.2090.2) in (A) medial and (B) lateral views; right ischium (MB.R.2090.4) in (C) lateral view; right ischium (MB.R.2090.3) in (D) medial and (E) lateral views. Abbreviations: amb, ambiens process; ia, ischiadic articulation; obf, obturator foramen; rtfi, ridge for M. flexor tibialis internus III. Scale bar $=200 \mathrm{~mm}$ [intended for double column width].

Figure 10. Tendaguru Formation Quarry $\mathrm{P}$ excavation sketch of Janenschia robusta referred material. Remaining material in the MfN is infilled with grey. Roman numerals refer to the four individuals designated by Bonaparte et al. (2000), and the other numbers correspond to the $P$ Quarry field numbers assigned by Janensch (see text for more details). Redrawn from Bonaparte et al. (2000), based on the field notebook of Janensch. Scale bar $=1000 \mathrm{~mm}$ [intended for double column width].

Figure 11. Janenschia robusta Quarry $P$ left humerus (MB.R.2095.7) in (A) proximal, (B) distal, (C) anterior, (D) medial, (E) posterior, and (F) lateral views. Abbreviations: acf, anconeal fossa; ddc, divided distal condyle; dpc, deltopectoral crest; pp, posterior process. Scale bar $=200 \mathrm{~mm}$ [intended for double column width].

Figure 12. Janenschia robusta Quarry P left radius (MB.R.2095.9) in (A) proximal, (B) distal, (C) anterior, and (D) posterior views. Abbreviations: bde, bevelled distal end; $\mathrm{mp}$, medial process; plr, posterolateral ridge. Scale bar $=200 \mathrm{~mm}$ [intended for single column width].

Figure 13. Janenschia robusta Quarry P left ulnae: MB.R.2095.8 in (A) anterior, (B) medial, (C) posterior, (D) lateral, (E) proximal, and (F) distal views; MB.R.2095.11 in (G) proximal, (H) distal, (I) anterior, (J) medial, (K) posterior, and (L) lateral views. Abbreviations: alp, anterolateral process; amp, anteromedial process; conp, concave profile; op, olecranon process; pex, posterior expansion; ras, radial articular surface. Scale bar $=200 \mathrm{~mm}$ [intended for double column width]. 
Figure 14. Janenschia robusta Quarry P carpometacarpus: left carpal (MB.R.2095.10) in (A) proximal/dorsal, (B) distal/ventral, (C) anterior, and (D) posterior views; left manual ungual (MB.R.2095.13) in (E) proximal and (F) medial views (dorsal margin at top); manual phalanx (MB.R.2245) in (G) proximal (dorsal margin at top) and (H) dorsal (proximal margin at left) views. Scale bar $=100 \mathrm{~mm}$ [intended for double column width].

Figure 15. Janenschia robusta Quarry $P$ left femur (MB.R.2707) in (A) proximal (anterior margin at top), (B) distal, (C) anterior, (D) medial, (E) posterior, and (F) lateral views. Abbreviations: 4tr, fourth trochanter; dac, distal anterior concavity; fc, fibular condyle; lb, lateral bulge; md, medial deflection; tc, tibial condyle. Scale bar $=200 \mathrm{~mm}$ [intended for double column width].

Figure 16. Janenschia robusta Quarry P left tibiae: MB.R.2095.1 in (A) proximal, (B) anterior, (C) posterior, and (D) distal views; MB.R.2095.4 in (E) proximal (anterior margin at top), (F) anterior, $(\mathrm{G})$ posterior, and $(\mathrm{H})$ distal views. The anterior margin is at the top in all proximal and distal views. Abbreviations: $c c$, cnemial crest; scc, second cnemial crest. Scale bar $=200$ $\mathrm{mm}$ [intended for double column width].

Figure 17. Janenschia robusta Quarry P left fibulae: MB.R.2095.2 in (A) anterior, (B) medial, (C) posterior, (D) lateral, (E) proximal, and (F) distal views; MB.R.2095.5 in (G) medial view. The anterior margin is at the top in the proximal and distal views. Scale bar $=200 \mathrm{~mm}$ [intended for double column width].

Figure 18. Janenschia robusta Quarry $P$ left astragalus (MB.R.2095.6) in (A) anterior, (B) medial, (C) posterior, (D) lateral, (E) dorsal/proximal, and (F) ventral/distal views. Abbreviations: If, lateral fossa; $p p$, posterior process. Scale bar $=100 \mathrm{~mm}$ [intended for double column width].

Figure 19. Tendaguria tanzaniensis holotypic dorsal vertebra 2 (MB.R.2092.2) in (A) anterior (slightly oblique), (B) left lateral, (C) posterior, (D) right lateral, (E) dorsal, and (F) ventral views. Abbreviations: acl, accessory lamina; acr, accessory ridge; afos, anterior fossa; CDF, centrodiapophyseal fossa; CPOL, centropostzygapophyseal lamina; CPRL, centroprezygapophyseal lamina; dfos, dorsal fossa; dp, diapophysis; epi, epipophysis; Ipf, lateral pneumatic foramen; ISPRL, lateral spinoprezygapophyseal lamina; mSPRL, medial spinoprezygapophyseal lamina; ns, neural spine; PCDL, posterior centrodiapophyseal lamina; PODL, postzygodiapophyseal lamina; posf, postspinal fossa; poz, postzygapophysis; pp, parapophysis; PPDL, paradiapophyseal lamina; PRCDF, prezygapophyseal centrodiapophyseal fossa; PRDL, prezygodiapophyseal lamina; PRSL, prespinal lamina; prz, prezygapophysis; SPOL, spinopostzygapophyseal lamina; TPOL, interpostzygapophyseal lamina; TPRL, interprezygapophyseal lamina. Scale bar $=200 \mathrm{~mm}$ [intended for double column width].

Figure 20. Tendaguria tanzaniensis holotypic dorsal vertebra 3 (MB.R.2092.1) in (A) anterior (slightly oblique), (B) left lateral, (C) posterior, (D) right lateral, (E) dorsal, and (F) ventral views. Abbreviations: acr, accessory ridge; afos, anterior fossa; divr, dividing ridge; epi, epipophysis; Ipf, lateral pneumatic foramen; PCDL, posterior centrodiapophyseal lamina; PODL, postzygodiapophyseal lamina; POSL, postspinal lamina; pp, parapophysis; PRCDF, prezygapophyseal centrodiapophyseal fossa; PRDL, prezygodiapophyseal lamina; PRSL, 
prespinal lamina; SDF, spinodiapophyseal fossa; SPDL, spinodiapophyseal lamina. Scale bar = $200 \mathrm{~mm}$ [intended for double column width].

Figure 21. C-T scan images of Tendaguria tanzaniensis holotypic dorsal vertebrae (MB.R.2092.2 and 2092.1). Part A is a reconstruction of MB.R.2092.1 in left lateral view, showing the positions of transverse cross-sections that are illustrated in posterior view (Parts B-E). Part F is a reconstruction of MB.R.2092.2 in left lateral view, showing the positions of transverse cross-sections that are illustrated in posterior view (Parts $G-M$ ). Scale bars $=100 \mathrm{~mm}$ [intended for double column width].

Figure 22. cf. Tendaguria cervical vertebra (MB.R.2091.31) in (A) anterior, (B) left lateral, (C) posterior, (D) right lateral, (E) dorsal, and (F) ventral views. Abbreviations: acr, accessory ridge; CPRL, centroprezygapophyseal lamina; epi, epipophysis; fos, fossa; Ipf, lateral pneumatic foramen; ns, neural spine; PCDL, posterior centrodiapophyseal lamina; $p p$, parapophysis; PRDL, prezygodiapophyseal lamina; SPRL, spinoprezygapophyseal lamina; vmr, ventral midline ridge. Scale bar $=100 \mathrm{~mm}$ [intended for double column width].

Figure 23. C-T scan images of cf. Tendaguria cervical vertebra (MB.R.2091.31). Part A is a reconstruction of MB.R.2091.31 in left lateral view, showing the positions of transverse cross-sections that are illustrated in posterior view (Parts $B-L)$. Part $M$ shows a medial crosssection, and parts $\mathrm{N}-\mathrm{P}$ show horizontal cross-sections illustrated in dorsal view. Scale bars = $50 \mathrm{~mm}$ [intended for double column width].

Figure 24. Wamweracaudia keranjei gen. et sp. nov holotypic caudal vertebrae (MB.R.2091.1-30) in anterior view. Roman numerals refer to their sequence as described in the text, with XXX and I representing the anteriormost and posteriormost preserved caudal vertebrae, respectively. Abbreviations: CPRL, centroprezygapophyseal lamina; cr, caudal rib; Iru, lateral rugosity; Itu, lower tuberosity; PRCDF, prezygapophyseal centrodiapophyseal fossa; PRDL, prezygodiapophyseal lamina; prsf, prespinal fossa; prsl, prespinal lamina; prsr, prespinal rugosity; prz, prezygapophysis; SDF, spinodiapophyseal fossa; SPRL, spinoprezygapophyseal lamina; utu, upper tubercle. Scale bar $=100 \mathrm{~mm}$ [intended for double column width].

Figure 25. Wamweracaudia keranjei gen. et $\mathrm{sp}$. nov holotypic caudal vertebrae (MB.R.2091.1-30) in left lateral view. Roman numerals refer to their sequence as described in the text, with XXX and I representing the anteriormost and posteriormost preserved caudal vertebrae, respectively. Abbreviations: hlr, horizontal ridge; Iru, lower rugosity; pdlr, posterodorsal lateral ridge; plgr, posterolateral groove; PRDL, prezygodiapophyseal lamina. Scale bar $=100 \mathrm{~mm}$ [intended for double column width].

Figure 26. Wamweracaudia keranjei gen. et $\mathrm{sp}$. nov holotypic caudal vertebrae (MB.R.2091.1-30) in posterior view. Roman numerals refer to their sequence as described in the text, with XXX and I representing the anteriormost and posteriormost preserved caudal vertebrae, respectively. Abbreviations: hyp, hyposphene; Iru, lateral rugosity; ISPOL, lateral spinopostzygapophyseal lamina; Itu, lower tubercle; mSPOL, medial spinopostzygapophyseal lamina; plgr, posterolateral groove; posf, postspinal fossa; posr, postspinal rugosity; SPOL, spinopostzygapophyseal lamina; utu, upper tubercle. Scale bar $=100 \mathrm{~mm}$ [intended for double column width]. 
Figure 27. Wamweracaudia keranjei gen. et sp. nov holotypic caudal vertebrae (MB.R.2091.1-30) in right lateral view. Roman numerals refer to their sequence as described in the text, with XXX and I representing the anteriormost and posteriormost preserved caudal vertebrae, respectively. Abbreviations: acjr, arch-centrum junction ridge; acr, anteriorly curving rib; adb, anterodorsal bulge; CPRL, centroprezygapophyseal lamina; hlr, horizontal lateral ridge; Ifos, lateral fossa; Iru, lateral rugosity; Itu, lower tubercle; pdb, posterodorsal bulge; pdlr, posterodorsal lateral ridge; plgr, posterolateral groove; posr, postspinal rugosity; PRDL, prezygodiapophyseal lamina; prsr, prespinal rugosity; prz, prezygapophysis; SDF, spinodiapophyseal fossa; SPOL, spinopostzygapophyseal lamina; SPRL, spinoprezygapophyseal lamina; utu, upper tubercle. Scale bar $=100 \mathrm{~mm}$ [intended for double column width].

Figure 28. Wamweracaudia keranjei gen. et sp. nov holotypic caudal vertebrae (MB.R.2091.1-30) in ventral view. Roman numerals refer to their sequence as described in the text, with XXX and I representing the anteriormost and posteriormost preserved caudal vertebrae, respectively. Abbreviations: $\mathrm{ACDL}$, anterior centrodiapophyseal lamina; acf, anterior chevron facet; acr, anteriorly curving rib; con, condyle; $c r$, caudal rib; ns, neural spine; pcf, posterior chevron facet; PRDL, prezygodiapophyseal lamina; prz, prezygapophysis; vf, vascular foramen; vmr, ventral midline ridge. Scale bar $=100 \mathrm{~mm}$ [intended for double column width].

Figure 29. Wamweracaudia keranjei gen. et $\mathrm{sp}$. nov holotypic caudal vertebrae (MB.R.2091.24, 21, 16) in posterodorsal view, showing the foramen on the neural canal floor. Roman numerals refer to their sequence as described in the text. Abbreviations: for, foramen. Scale bar $=50 \mathrm{~mm}$ [intended for double column width].

Figure 30. Wamweracaudia keranjei gen. et sp. nov holotypic chevrons (MB.R.2091): G28 in (A) proximal, (B) anterior, (C) left lateral, and (D) posterior views; G27 in (E) proximal, (F) anterior, $(\mathrm{G})$ left lateral, and $(\mathrm{H})$ posterior views. Abbreviations: amr, anterior midline ridge; hc, haemal canal; pbr, proximal bridge; pmr, posterior midline ridge; rs, roller surface. Scale bar $=100 \mathrm{~mm}$ [intended for double column width].

Figure 31. Wamweracaudia keranjei gen. et sp. nov referred caudal vertebra (MB.R.2094) from Quarry Oa in (A) anterior, (B) left lateral, (C) posterior, (D) right lateral, (E) dorsal, and (F) ventral views. Abbreviations: acr, anteriorly curving rib; hyp, hyposphene; Itu, lower tubercle; plgr, posterolateral groove; posf, postspinal fossa; SPOL, spinopostzygapophyseal lamina; SPRL, spinoprezygapophyseal lamina; utu, upper tubercle. Scale bar $=200 \mathrm{~mm}$ [intended for double column width].

Figure 32. Eusauropoda indet. Nr. 5 right metacarpals I-V (MB.R.2093) in (A) proximal, (B) dorsal/anterior, (C) medial, (D) ventral/posterior, (E) lateral, and (F) distal views. Dorsal/anterior margin at top in parts $\mathrm{A}$ and $\mathrm{F}$; proximal end at top in parts B-E. Scale bar = $100 \mathrm{~mm}$ [intended for double column width].

Figure 33. Eusauropoda indet. Nr. 5 right manual phalanges (MB.R.2093): phalanx I-1 in (A) proximal, (B) distal, and (C) dorsal/anterior views; ungual I-2 in (D) proximal, (E) medial, and 
(F) lateral views. Dorsal/anterior margin at top in parts A, B, D-F; proximal end at top in part C. Scale bar $=100 \mathrm{~mm}$ [intended for double column width].

Figure 34. Eusauropoda indet. Nr. 5 right manus (MB.R.2093) articulated in proximal view. Scale bar $=100 \mathrm{~mm}$ [intended for single column width].

Figure 35. Eusauropoda indet. Nr. 5 right manus (MB.R.2093) in articulation in (A) anterior, (B) medial, and (C) lateral views. Scale bar $=100 \mathrm{~mm}$ [intended for single column width].

Figure 36. Eusauropoda indet. Quarry G right ischium (MB.R.2091.32) in medial view. Abbreviations: gr, groove; rtfi, ridge for M. flexor tibialis internus III. Scale bar $=200 \mathrm{~mm}$ [intended for single column width].

Figure 37. Eusauropoda indet. Quarry IX right radius (MB.R.2096.2) in (A) proximal, (B) distal, (C) anterior, (D) medial, (E) posterior, and (F) lateral views. Abbreviations: bde, bevelled distal end; $\mathrm{mp}$, medial process; plr, posterolateral ridge; $\mathrm{pr}$, posterior ridge. Scale bar $=200$ $\mathrm{mm}$ [intended for double column width].

Figure 38. Eusauropoda indet. Quarry IX right femur (MB.R.2096.1) in (A) anterior, (B) medial, (C) posterior, and (D) lateral views. Abbreviations: 4tr, fourth trochanter; dac, distal anterior concavity; fc, fibular condyle; lb, lateral bulge; md, medial deflection; tc, tibial condyle. Scale bar $=200 \mathrm{~mm}$ [intended for double column width].

Figure 39. C-T scan images of Australodocus bohetii holotypic cervical vertebra (MB.R.2455). Part $A$ is a $3 D$ reconstruction of the vertebra in left lateral view, showing the positions of transverse cross-sections that are illustrated in posterior view (Parts $\mathrm{B}-\mathrm{L}$ ). Part $\mathrm{M}$ shows a medial, longitudinal cross-section, viewed from the right side of the vertebra. Scale bars $=50$ $\mathrm{mm}$ [intended for double column width].

Figure 40. C-T scan images of Australodocus bohetii paratypic cervical vertebra (MB.R.2454) Part $A$ is a $3 D$ reconstruction of the vertebra in left lateral view, showing the positions of transverse cross-sections that are illustrated in posterior view (Parts $B-I$ ). Parts $K$ and $L$ show longitudinal cross-sections, viewed from the left side of the vertebra. Scale bars $=50 \mathrm{~mm}$ [intended for double column width].

Figure 41. Strict consensus cladogram of equal weights analysis, with seven taxa excluded $a$ priori (see text for details). Janenschia, Tendaguria and Wamweracaudia are highlighted in red, and the approximate position of Australodocus is marked with a red star [intended for double column width].

Figure 42. Strict consensus cladogram of extended implied weights analysis, with seven taxa excluded a priori (see text for details). Janenschia, Tendaguria and Wamweracaudia are highlighted in red, and the approximate position of Australodocus is marked with a red star [intended for double column width].

Figure 43. Time-calibrated phylogenetic agreement subtree, based on equal weights analysis. Taxon ranges include both true stratigraphic range and uncertainty. The somphospondylan titanosauriform clade has been collapsed. Note that Lingwulong might be 
as old as late Early Jurassic, but we restrict it to the early Middle Jurassic in this plot for simplicity. Lapparentosaurus, from the Middle Jurassic of Madagascar, is included as an African taxon, for simplicity, given that these palaeocontinents were still connected at this time. Silhouettes of dinosaurs drawn by Scott Hartman, Mike Taylor and Mathew Wedel, and available at Phylopic (http://phylopic.org/) under a Creative Commons AttributionNonCommercial-ShareAlike 3.0 Unported license (https://creativecommons.org/licenses/bync-sa/3.0/) [intended for double column width].

Figure 44. Time-calibrated phylogenetic agreement subtree, based on extended implied weights analysis [intended for double column width].

Figure 45. Paleogeographic reconstruction showing the main Middle Jurassic (MJ), Late Jurassic (LJ), and Early Cretaceous (EK) sauropod faunas discussed in text. Palaeogeographic reconstruction of $150 \mathrm{Ma}$ from Fossilworks (http://fossilworks.org/) [intended for double column width]. 


\section{APPENDIX 1 - REVISED CHARACTERS AND SCORES}

We realised that two characters (C84, C300) from previous iterations of our data matrix are problematic, and thus they have been replaced, as follows:

Character 84, pertaining to whether or not the parietal has an elongate posterolateral process, does not seem to truly capture any genuine morphological variation and is replaced with the following character:

C84. Frontal, position of parietal suture relative to supratemporal fenestra: close to anterior margin of the fenestra (0); at approximately the centre of the fenestra or more posteriorly (1) (Tschopp \& Mateus, 2013).

Character 300 is a near-duplication of C88 (both pertaining to the relative positions of the orbit and lateral temporal fenestra). As such, we have replaced our existing C300 with the following character:

C300. External nares, position: retracted to level of orbit, facing laterally (0); retracted to position between orbits, facing dorsally or dorsolaterally (1) (McIntosh, 1990; Upchurch, 1995; Whitlock, 2011a).

We have also revised 21 of the characters from previous iterations of the matrix, as follows:

C26. Anteriormost caudal centra, lowest average Elongation Index (aEl; centrum anteroposterior length [excluding articular ball] divided by the mean average value of the anterior surface mediolateral width and dorsoventral height) value of: less than $0.6(0) ; 0.6$ or greater (1) (Gauthier, 1986; Upchurch, 1995, 1998; Upchurch et al., 2004; Mannion et al., 2013; revised here to only include the anteriormost caudal vertebrae).

C27. Anterior caudal centra, anteroposterior length of posterior condylar ball to mean average radius ([mediolateral width + dorsoventral height] divided by 4 ) of anterior articular surface of centrum ratio: zero (posterior articular surface of centrum is flat or concave) (0); less than or equal to 0.3 (posterior articular surface of centrum is mildly convex) (1); greater than 0.3 (posterior articular surface of centrum is strongly convex) (2); greater than 0.6 (posterior articular surface of centrum is very strongly convex) (3) (McIntosh, 1990; Upchurch, 1995, 1998; Salgado et al., 1997; Wilson, 2002; Whitlock et al., 2011; Mannion et al., 2013; note that the highest value for a taxon is always used; revised here by adding an extra state to characterize taxa with extremely prominent posterior condyles) [ordered].

C33. Anterior caudal neural spines, mediolateral width to anteroposterior length ratio at base of spine (dorsal to zygapophyses): less than 1.0 (0); 1.0 or greater (1) (Upchurch, 1998; Mannion et al., 2013; revised here so that mediolateral width is measured at the same point as anteroposterior length).

C40. Humerus to femur proximodistal length ratio: 0.7 or less $(0) ;>0.7$ to $<0.8(1) ; 0.8$ to $<0.9$ (2); 0.9-0.95 (3); >0.95 (4) (Wilson, 2002; Upchurch et al., 2004; Tschopp et al., 2015a; Poropat et al., 2016; Mannion et al., 2017; an additional state has been added for taxa with ratios of 0.7 or less) [ordered]. 
C56. Manual ungual on digit I to metacarpal I proximodistal length ratio: 0.5 or greater (0); less than 0.5 (1) (Upchurch et al., 2004; Mannion et al., 2013; revised here so that taxa that have lost their manual phalanges [derived state for C242] are scored as a "?").

C98. Basioccipital, foramen or pit on the posterior surface of the basal tubera: absent (0); present (1) (Wilson, 2002; Mannion, 2011; Mannion et al., 2013; revised here to clarify character statement following Tschopp et al., 2015a).

C100. Basal tubera, posterior surface bordered laterally and ventrally by a raised, thickened lip: absent (0); present (1) (Wilson, 2002, 2005b; modified based on Mannion, 2011).

C140. Cervical ribs, longest shafts extend beneath: fewer than 3 vertebrae (0); 3 vertebrae or more (1) (Wedel et al., 2000; Mannion et al., 2013); revised here so that taxa with short ribs [scored with the plesiomorphic state for C139] are scored as a "?").

C160. Anterior dorsal neural spines, orientation of anterior margin in lateral view: inclined anteriorly or vertical (0); posterodorsally inclined (1) (Upchurch et al., 2004; Mannion et al., 2013; revised here to clarify character statement).

C163. Middle-posterior dorsal neural spines, with triangular aliform processes: weakly developed aliform processes (0); strongly developed triangular aliform processes so that the lateral tips of these processes extend further laterally than the postzygapophyses (1) (Upchurch, 1998; Wilson \& Sereno, 1998; Wilson, 2002; Upchurch et al., 2004; revised here so that only taxa with aliform processes scored for this character).

C177. Anterior caudal centra with posterior convexity: convex in anteriormost caudal vertebrae, changing to flat or concave in more distal anterior caudal vertebrae (0); convex throughout all anterior caudal vertebrae with ribs (1) (Mannion et al., 2013; revised here to only include taxa with some procoely in the anterior caudal sequence - taxa lacking procoelous anterior caudal vertebrae are scored as a "?").

C181. Anterior caudal centra (excluding the anteriormost caudal vertebrae), ventral longitudinal hollow: absent (0); present (1) (McIntosh, 1990; Upchurch, 1995, 1998; Wilson, 2002; revised here to separate anterior and middle caudal vertebrae).

C182. Anterior caudal centra (excluding the anteriormost caudal vertebrae), distinct ventrolateral ridges, extending the full length of the centrum: absent (0); present (1) (McIntosh, 1990; Upchurch, 1995, 1998; Upchurch et al., 2004; Mannion et al., 2013; revised here to separate anterior and middle caudal vertebrae).

C198. Anterior caudal neural spines, spinoprezygapophyseal lamina (SPRL)spinopostzygapophyseal lamina (SPOL) contact: absent (0); present, forming a prominent lateral lamina on the neural spine (1) (Wilson, 1999, 2002; revised here so that only taxa in which the SPRL extends onto the lateral surface are scored).

C206. Anterior caudal ribs: do not extend beyond posterior end of centrum (excluding posterior ball) (0); extend beyond posterior end of centrum (excluding posterior ball) (1) (Mannion \& Calvo, 2011; Mannion et al., 2013; revised here so that only taxa with posterolaterally directed caudal ribs are scored for this character).

C254. Ischium, long-axis of shaft, if projected upwards/proximally: passes through the lower part of the acetabular margin or the upper part of the pubic articular surface (i.e. it is approximately $60^{\circ}$ to the horizontal in lateral view) (0); passes through the upper part of the acetabular margin or even approaches the rim of the iliac articulation (i.e. the shaft is at approximately $80^{\circ}$ to the horizontal) (1); passes through the lower part of the pubic articular surface (i.e. it is approximately horizontally oriented), such that the posterior margin of the iliac peduncle and the dorsal margin of the shaft form a right angle in lateral view (2) (Upchurch, 1995, 1998; Wilson \& Sereno, 1998; revised here to add an extra state) [unordered]. 
C266. Astragalus, ascending process: limited to anterior two-thirds of astragalus (0); extends beyond anterior two-thirds of astragalus (usually to the posterior margin) (1) (Wilson \& Sereno, 1998; Wilson, 2002; revised here following Tschopp et al., 2015a).

C297. Frontal, anteroposterior length to transverse width ratio: 1.0 or greater (0); less than 1.0 and > 0.5 (1); 0.5 or less (2) (Whitlock, 2011a; Tschopp \& Mateus, 2013; Poropat et al., 2016; revised here to add an extra state) [ordered].

C348. Sacral neural spines, all fused, forming a dorsal 'platform': absent (0); present (1) (Martínez et al., 2004; Poropat et al., 2016; revised here so that only taxa with the derived state for $\mathrm{C} 174$ are scored).

C350. Anterior-middle caudal centra (excluding Cd1), comparison of anterior and posterior articular faces of amphicoelous centra: anterior face more concave than posterior one, or these two faces are equally concave (0); posterior face more deeply concave than anterior face (1) (González Riga et al., 2009; Carballido et al., 2012; D'Emic et al., 2013; Poropat et al., 2016; revised here to only score for taxa with at least some amphicoelous caudal centra in the anterior-middle caudal sequence).

C373. Metacarpals, longest metacarpal to radius proximodistal length ratio: less than 0.50 (0); 0.50 or greater (1) (Poropat et al., 2016; revised here so that only taxa scored as a "1" for C52 are scored).

Taxon scores for C1-423 follow those in the data matrix of González Riga et al. (2018), whilst scores for C1-416 for Moabosaurus, Mierasaurus and Soriatitan follow those of Royo-Torres et al. $(2017 a, b)$, with the following changes made (the first number denotes the character and the number/symbol in parentheses denotes the new score):

Shunosaurus: 140 (?); 163 (?); 177 (?); 198 (?); 206 (?); 348 (?); 373 (?)

Omeisaurus: 40 (2); 163 (?); 177 (?); 198 (?); 348 (?); 373 (?)

Mamenchisaurus: 27 (3); 40 (1); 177 (1); 198 (?); 206 (?); 212 (1); 233 (1)

Camarasaurus: 11 (0/1); 33 (0); 40 (1); 118 (0/1); 177 (?); 198 (?); 206 (?); 254 (2); 300 (0); $348(?)$

Nigersaurus: 16 (0); 140 (?); 149 (0); 150 (0); 180 (0); 181 (?); 182 (?); 249 (1); 313 (0)

Apatosaurus: 3 (0); 140 (?); 163 (?); 177 (0); 206 (?); 258 (0); 348 (?)

Diplodocus: 99 (0); 140 (?); 163 (?); 177 (0); 206 (?); 348 (?); 373 (?)

Abydosaurus: 177 (?)

Alamosaurus: 27 (3); 56 (?); 177 (1); 198 (?); 350 (?)

Andesaurus: 163 (?); 177 (0); 198 (?)

Aragosaurus: 40 (2); 177 (?); 198 (?); 206 (?)

Astrophocaudia: 177 (?); 198 (?)

Atlasaurus: 40 (4); 373 (?)

Baotianmansaurus: 177 (?)

Brachiosaurus: 40 (4); 177 (?); 198 (?); 206 (?); 348 (?)

Cedarosaurus: 40 (4); 177 (?); 198 (?)

Chubutisaurus: 40 (2); 177 (?)

Cloverly titanosauriform: 163 (?)

Daxiatitan: 27 (3); 198 (?); 206 (?)

Dongbeititan: 27 (3); 163 (?); 198 (?)

Dongyangosaurus: 163 (?); 177 (0); 198 (?)

Euhelopus: 300 (0); 348 (?)

Europasaurus: 177 (?); 198 (?); 348 (?) 
Vouivria: 40 (3); 177 (?)

Galveosaurus: 177 (?)

Giraffatitan: 40 (4); 177 (?); 198 (?); 300 (0); 348 (?)

Wamweracaudia: 27 (3); 177 (0); 198 (?); 206 (?)

Huanghetitan: 177 (?)

Huanghetitan ruyangensis: 177 (?); 198 (?)

Janenschia: 60 (0)

Jiangshanosaurus: 198 (?)

Lapparentosaurus: 163 (?); 177 (?); 198 (?)

Ligabuesaurus: 40 (2); 163 (?)

Lusotitan: 177 (?); 198 (?)

Malarguesaurus: 26 (?); 177 (?); 180 (0); 181 (0); 198 (?)

Malawisaurus: 27 (3); 33 (0); 163 (?); 177 (1); 198 (?)

Opisthocoelicaudia: 40 (1); 56 (?); 160 (1); 177 (?); 198 (?); 259 (0); 350 (?)

Paluxysaurus: 40 (2); 50 (?); 51 (?); 148 (1/2); 163 (?); 177 (?); 198 (?)

Phuwiangosaurus: 40 (1); 100 (1); 177 (?); 198 (?); 348 (?)

Rapetosaurus: 26 (?); 27 (3); 40 (1); 56 (?); 177 (1); 182 (1); 198 (?); 350 (?)

Saltasaurus: 27 (3); 177 (1); 198 (?); 350 (?)

Sonorasaurus: 177 (?)

Tangvayosaurus: 163 (?); 177 (?); 198 (?)

Tastavinsaurus: 25 (1); 27 (0); 60 (0/1); 61 (0/1); 71 (0/1); 163 (?); 177 (?); 178 (0/1); 179 (0);

$180(0) ; 195(1 / 2) ; 198($ ?); $249(0) ; 256(0)$

Tehuelchesaurus: 40 (1); 163 (?)

Tendaguria: 17 (?); 18 (?); 118 (?); 119 (?); 120 (?); 121 (?); 122 (?); 123 (?); 124 (?); 125 (?);

126 (?); 127 (?); 128 (?); 129 (?); 130 (?); 131 (?); 135 (?)

Venenosaurus: 36 (?); 177 (?); 198 (?)

Xianshanosaurus: 27 (3); 177 (1); 198 (?); 206 (?); 350 (?)

Epachthosaurus: 27 (3); 40 (2); 56 (?); 177 (1); 181 (1); 198 (?); 350 (?)

Nemegtosaurus: 25 (1); 65 (1); 66 (1); 68 (0); 71 (1); 256 (1); 258 (0); 259 (0); 265 (1); 268 (1); 269 (1); 388 (1); 389 (0); 393 (0); 394 (1)

Tapuiasaurus: 163 (?)

Diamantinsaurus: 40 (1)

Savannasaurus: 177 (?); 198 (?)

Wintonotitan: 177 (?); 198 (?)

Futalognkosaurus: 27 (3); 40 (1); 163 (?); 177 (1); 198 (?); 350 (?)

Muyelensaurus: 27 (3); 33 (0); 100 (1); 177 (1); 198 (?); 350 (?)

Aeolosaurus: 27 (3); 177 (1); 350 (?)

Isisaurus: 27 (3); 163 (?); 177 (1); 198 (?); 206 (?); 350 (?)

Jobaria: 40 (1); 64 (1); 163 (?); 177 (?); 181 (1); 182 (1); 198 (?); 348 (?); 373 (?)

Padillasaurus: 177 (?); 198 (?)

Losillasaurus: 49 (0); 100 (1); 132 (0); 141 (0); 198 (?)

Turiasaurus: 18 (0); 19 (1); 44 (0); 72 (1); 134 (0); 141 (0); 174 (0); 193 (?); 300 (0); 348 (?); 373 (?)

Zby: 373 (?)

Mendozasaurus: 27 (3); 177 (1); 350 (?)

Notocolossus: 27 (3); 198 (?); 350 (?)

Pitekunsaurus: 27 (3); 160 (1); 163 (?); 177 (1); 198 (?); 350 (?)

Rinconsaurus: 27 (3); 177 (0); 198 (?) 
Patagotitan: 27 (3); 177 (0); 198 (?)

Soriatitan: 30 (?); 31 (?); 33 (0); 40 (?); 44 (?); 66 (0); 108 (1); 114 (?); 173 (1); 177 (?); 178

(1); 179 (0); 181 (1); 185 (?); 193 (0); 195 (0/1); 198 (?); 231 (?); 232 (?); 353 (?); 410 (1)

Moabosaurus: 3 (1); 9 (?); $16(0 / 1) ; 22(0 / 1) ; 27(3) ; 28(0) ; 30(0) ; 33(0) ; 35(0) ; 43(1) ; 83$ (0); 85 (0); 87 (1); 98 (0); $100(0 / 1) ; 106(0) ; 116(0 / 1) ; 120$ (0/1); 122 (1/2); 133 (0); $150(1)$; 151 (0); 155 (0/1); 167 (0/1); 168 (0); 170 (0); 177 (1); 183 (0); 194 (0); 201 (0); 203 (0); 205 (1/2); 206 (0); $210(0) ; 211(0) ; 221(0) ; 224$ (1); 228 (0); $230(0) ; 233$ (1); 255 (1); $256(0)$; 257 (0); 280 (?); 281 (?); 299 (1); 302 (0); 303 (0); 304 (0); 308 (0); 309 (1); 311 (0); 312 (0); 313 (0); 314 (1); 315 (0); 316 (0); 317 (1); 318 (0); 321 (0); 322 (1); 329 (1); 333 (0); 340 (0); 344 (0/1); 345 (1); 346 (1); 349 (0); 351 (0); 352 (0); 354 (0); 355 (0); $356(0) ; 357$ (0); 369 (0); $398(0) ; 399$ (1); 402 (0/1); 405 (1); $408(0)$

Mierasaurus: 13 (1); 21 (1); 25 (1); 26 (0); 27 (3); 30 (0); 33 (0); 34 (0); 37 (1); 52 (0); 54 (?); 59 (0); 67 (1); 68 (1); 70 (0); 71 (1); 79 (?); 85 (0); 101 (0); 108 (0/1); 117 (?); 120 (1); 121 (1); 133 (?); 138 (?); 151 (0); 155 (0); 170 (0); 171 (?); 177 (?); 183 (1); 184 (0); 186 (?); 194 (0); 195 (0); 197 (0); 199 (0); 200 (1); 214 (1); 236 (0); 244 (1); 250 (1); 255 (1); 258 (?); 259 (2); 260 (?); 261 (?); 266 (1); 271 (?); 272 (0); 274 (0); 280 (?); 281 (?); 284 (0); 297 (1); 298 (0); 299 (0); 300 (0); 303 (0); 304 (0); 311 (0); 314 (1); 315 (0); $316(0) ; 317$ (1); 318 (0); 329 (1); 333 (0); 340 (0); 344 (1); 345 (1); 348 (?); 351 (?); 352 (0); 353 (0); 357 (0); 383 (0); 388 (0); 396 (0); 397 (1); 402 (0/1); 409 (1); $410(0) ; 411$ (0)

\section{APPENDIX 2 - CHARACTER LIST}

Characters 1-423 are the same as those presented in González Riga et al. (2018), except for replaced and revised characters (see Appendix 1). Characters 424-542 are newly added to this matrix.

C1. Premaxillary anterior margin, shape: without step or with anteroposteriorly short 'muzzle', less than 0.25 of skull length (measured up to the anterior point of the ascending process of premaxilla) (0); elongate, boot-shaped snout, equal to or greater than 0.25 of skull length (1) (Wilson, 2002; Mannion et al., 2013).

C2. External naris, greatest diameter to greatest diameter of orbit ratio: greater than $1.0(0)$; 1.0 or less (1) (Mclntosh, 1990; Upchurch, 1995; Upchurch et al., 2004; Mannion et al., 2013).

C3. Parietal occipital process, dorsoventral height to greatest diameter of foramen magnum ratio: greater than 1.0 (0); 1.0 or less (1) (Wilson, 2002; Mannion et al., 2013).

C4. Parietal, distance separating supratemporal fenestrae to long axis of supratemporal fenestra ratio: 1.0 or greater (0); less than 1.0 (1) (Wilson, 2002; Mannion et al., 2013).

C5. Quadratojugal, anterior process to dorsal process length ratio: 1.3 or less (0); greater than 1.3 (1) (Upchurch, 1998; Wilson \& Sereno, 1998; Mannion et al., 2013).

C6. Supraoccipital, dorsoventral height to foramen magnum dorsoventral height ratio: 1.0 or greater (0); less than 1.0 (1) (Wilson, 2002; Mannion et al., 2013).

C7. Occipital condyle, dorsoventral height to combined occipital condyle + basal tubera dorsoventral height ratio: less than 0.6 (0); 0.6 or greater (1) (Mannion, 2011; Mannion et al., 2013).

C8. Basal tubera, mediolateral width to occipital condyle mediolateral width: less than 1.5 (0); 1.5 or greater (1) (Wilson, 2002; Mannion, 2011; Mannion et al., 2013). 
C9. Basipterygoid processes, length to basal diameter ratio: less than 3.0 (0); 3.0 or greater (1) (Wilson, 2002; Upchurch et al., 2004; Mannion et al., 2013; length is measured up to the base of the basal tubera).

C10. Surangular, dorsoventral height to maximum dorsoventral height of angular ratio: 2.0 or greater (0); less than 2.0 (1) (Wilson \& Sereno, 1998; Upchurch et al., 2004; Mannion et al., 2013).

C11. Tooth crowns, Slenderness Index (SI) values (apicobasal length of the tooth crown divided by its maximum mesiodistal width): less than $2.0(0) ; 2.0$ to $<4.0$ (1); 4.0 or greater (2) (Upchurch, 1998; Upchurch et al., 2004; Mannion et al., 2013) [ordered].

C12. Maxillary teeth, number: 17 or more (0); fewer than 17 (1) (Mannion et al., 2013).

C13. Dentary teeth, number: greater than 15 (0); 15 or fewer (1) (Wilson \& Sereno, 1998; Mannion et al., 2013).

C14. Cervical vertebrae, number: 13 or fewer (0); 14-15 (1); more than 15 (2) (Upchurch, 1995, 1998; Wilson \& Sereno, 1998; Mannion et al., 2013) [ordered].

C15. Cervical centra, highest average Elongation Index value (aEl; centrum anteroposterior length [excluding articular ball] divided by the mean average value of the posterior articular surface mediolateral width and dorsoventral height) of: less than $3.0(0)$; between 3.0 and $<4.0$ (1); greater than 4.0 (2) (Upchurch, 1995, 1998; Upchurch et al., 2004; Chure et al., 2010) [ordered].

C16. Anterior cervical centra, posterior articular face dorsoventral height to mediolateral width ratio: 1.0 or greater (0); less than 1.0 (1) (Upchurch, 1998; Upchurch et al., 2004; Mannion et al., 2013).

C17. Middle-posterior cervical centra, posterior articular face dorsoventral height to mediolateral width ratio: 1.0 or less (0); greater than 1.0 (1) (Curry Rogers, 2005; Mannion et al., 2013).

C18. Posterior cervical neural arch to centrum dorsoventral height ratio, measured on anterior face of vertebra (arch height measured from dorsal surface of centrum to base of prezygapophyses): 0.5 or greater (0); less than 0.5 (1) (Bonaparte et al., 2006; Mannion et al., 2013).

C19. Posteriormost cervical and anteriormost dorsal neural spines, dorsoventral height divided by posterior centrum height: 1.0 or greater (0); less than 1.0 (1) (D'Emic, 2012; Mannion et al., 2013).

C20. Dorsal vertebrae, number: 13 or more (0); 12 or fewer (1) (McIntosh, 1990; Upchurch, 1998; Wilson \& Sereno, 1998; Upchurch et al., 2004).

C21. Anterior dorsal centra, posterior articular face mediolateral width to dorsoventral height ratio: less than 1.3 (0); 1.3 or greater (1) (Mannion et al., 2013).

C22. Middle-posterior dorsal centra, posterior articular face mediolateral width to dorsoventral height ratio: less than 1.0 (0); 1.0 or greater (1) (Upchurch, 1998; Upchurch et al., 2004; Mannion et al., 2013).

C23. Posterior dorsal neural spines, dorsoventral height divided by posterior centrum dorsoventral height: 1.0 or greater (0); less than 1.0 (1) (McIntosh, 1990; Upchurch, 1995, 1998; Mannion et al., 2013).

C24. Sacral vertebrae, number: 5 or fewer (0); 6 or more (1) (McIntosh, 1990; Upchurch, 1995, 1998; Wilson \& Sereno, 1998).

C25. Anterior caudal centra, mediolateral width to dorsoventral height (excluding chevron facets) of anterior surface ratio: less than 1.0 (0); 1.0 or greater (1) (Upchurch et al., 2004; Mannion et al., 2013). 
C26. Anteriormost caudal centra, lowest average Elongation Index (aEl; centrum anteroposterior length [excluding articular ball] divided by the mean average value of the anterior surface mediolateral width and dorsoventral height) value of: less than $0.6(0) ; 0.6$ or greater (1) (Gauthier, 1986; Upchurch, 1995, 1998; Upchurch et al., 2004; Mannion et al., 2013; revised here to only include the anteriormost caudal vertebrae).

C27. Anterior caudal centra, anteroposterior length of posterior condylar ball to mean average radius ([mediolateral width + dorsoventral height] divided by 4 ) of anterior articular surface of centrum ratio: zero (posterior articular surface of centrum is flat or concave) (0); less than or equal to 0.3 (posterior articular surface of centrum is mildly convex) (1); greater than 0.3 (posterior articular surface of centrum is strongly convex) (2); greater than 0.6 (posterior articular surface of centrum is very strongly convex) (3) (McIntosh, 1990; Upchurch, 1995, 1998; Salgado et al., 1997; Wilson, 2002; Whitlock et al., 2011; Mannion et al., 2013; note that the highest value for a taxon is always used; revised here by adding an extra state to characterize taxa with extremely prominent posterior condyles) [ordered].

C28. Middle caudal centra, mediolateral width to dorsoventral height (excluding chevron facets) of anterior surface ratio: less than 1.0 (0); 1.0 or greater (1) (Upchurch et al., 2004; Mannion et al., 2013).

C29. Middle caudal centra, average Elongation Index (aEl; centrum anteroposterior length [excluding articular ball] divided by the mean average value of the anterior surface mediolateral width and dorsoventral height [excluding chevron facets]) value: less than 1.4 (0); 1.4 or higher (1) (Upchurch \& Martin, 2003; Upchurch et al., 2004; Whitlock, 2011a; Mannion et al., 2013).

C30. Posterior caudal centra, mediolateral width to dorsoventral height (excluding chevron facets) of anterior surface ratio: less than 1.2 (0); 1.2 or greater (1) (Upchurch et al., 2004; Mannion et al., 2013).

C31. Posterior caudal centra, average Elongation Index (aEl; centrum anteroposterior length [excluding articular ball] divided by the mean average value of the anterior surface mediolateral width and dorsoventral height [excluding chevron facets]) value: less than 1.7 (0); 1.7 or higher (1) (Upchurch \& Martin, 2003; Upchurch et al., 2004; Whitlock, 2011a; Mannion et al., 2013).

C32. Anteriormost caudal neural spines, dorsoventral height divided by centrum height: 1.2 or greater (0); less than 1.2 (1) (McIntosh, 1990; Calvo \& Salgado, 1995; Upchurch, 1995, 1998; Mannion et al., 2013).

C33. Anterior caudal neural spines, mediolateral width to anteroposterior length ratio at base of spine (dorsal to zygapophyses): less than 1.0 (0); 1.0 or greater (1) (Upchurch, 1998; Mannion et al., 2013; revised here so that mediolateral width is measured at the same point as anteroposterior length).

C34. Anterior caudal neural spines, maximum mediolateral width to minimum mediolateral width ratio: less than 2.0 (0); 2.0 or greater (spines expand dorsally, forming 'club'- or 'mace'-shaped spinous processes) (1) (Canudo et al., 2008; Taylor, 2009; Mannion et al., 2013).

C35. Anterior chevrons (excluding first chevron), dorsoventral height of haemal canal divided by total chevron height: less than $0.40(0) ; 0.40$ or greater (1) (Curry Rogers \& Forster, 2001; Wilson, 2002; Mannion et al., 2013; note that dorsoventral height of the haemal canal is measured from the proximal tip of the chevron down to the distal tip of the haemal canal, regardless of whether the chevron is dorsally bridged). 
C36. Scapular acromion process, dorsoventral height to minimum dorsoventral height of scapular blade ratio: less than 3.0 (0); 3.0 or greater (1) (Wilson \& Sereno, 1998; Mannion et al., 2013; dorsoventral height is measured perpendicular to long-axis of scapular blade).

C37. Scapular blade, maximum (measured at or close to distal end) to minimum dorsoventral height ratio: 2.0 or greater (0); less than 2.0 (1) (Wilson, 2002; Rose, 2007; Mannion et al., 2013).

C38. Coracoid, anteroposterior length to dorsoventral height of scapular articulation ratio: 1.0 or greater (0); less than 1.0 (1) (Wilson, 2002; Mannion et al., 2013).

C39. Sternal plate, maximum length divided by humerus proximodistal length: less than 0.65 (0); 0.65 or greater (1) (McIntosh, 1990; Upchurch, 1998; Mannion et al., 2013).

C40. Humerus to femur proximodistal length ratio: 0.7 or less $(0) ;>0.7$ to $<0.8(1) ; 0.8$ to $<0.9$ (2); 0.9-0.95 (3); >0.95 (4) (Wilson, 2002; Upchurch et al., 2004; Tschopp et al., 2015a; Poropat et al., 2016; Mannion et al., 2017; an additional state has been added for taxa with ratios of 0.7 or less) [ordered].

C41. Humerus, maximum mediolateral width of proximal end divided by proximodistal length: 0.4 or greater (0); less than 0.4 (1) (Mannion et al., 2013).

C42. Humerus, minimum mediolateral width divided by proximodistal length: 0.15 or greater (0); less than 0.15 (1) (Curry Rogers, 2005; Mannion et al., 2013).

C43. Humerus shaft eccentricity, mediolateral to anteroposterior width ratio at midshaft: greater than 1.5 (usually close to 1.8$)(0) ; 1.5$ or lower (usually close to 1.3) (1) (Wilson, 2002; Mannion et al., 2012, 2013).

C44. Radius to humerus proximodistal length ratio: 0.65 or greater (0); less than 0.65 (1) (Yates \& Kitching, 2003; Mannion et al., 2013).

C45. Radius, maximum diameter of the proximal end divided by proximodistal length: less than 0.3 (0); 0.3 or greater (1) (McIntosh, 1990; Upchurch, 1995, 1998; Upchurch et al., 2004).

C46. Radius, mediolateral width of proximal to distal end ratio: 1.0 or greater (0); less than 1.0 (1) (Curry Rogers, 2005; Mannion et al., 2013; note that in taxa with a twisted radius, the dimension of the long axis of the distal end is used).

C47. Radius, distal end mediolateral width to midshaft mediolateral width ratio: less than 2.0 (0); 2.0 or greater (1) (Wilson, 2002; Rose, 2007; Mannion et al., 2013; note that in taxa with a twisted radius, the dimension of the long axis of the distal end is used).

C48. Radius, distal end mediolateral to anteroposterior width ratio: 1.5 or greater (0); less than 1.5 (1) (Wilson \& Sereno, 1998; Mannion et al., 2013).

C49. Radius, distal condyle orientation: perpendicular or bevelled less than $20^{\circ}$ to long axis of shaft (0); bevelled at least $20^{\circ}$ to long axis of shaft (1) (Curry Rogers \& Forster, 2001; Wilson, 2002; Mannion et al., 2013; note that in most taxa only the lateral half of the distal end is bevelled, but this is used as the measurement in those instances).

C50. Ulna, ratio of maximum mediolateral width of proximal end to ulna length: gracile, ratio is less than 0.4 (0); stout, ratio is 0.4 or greater (1) (Wilson, 2002; Curry Rogers, 2005; Mannion et al., 2013).

C51. Ulna, ratio of mediolateral width of proximal end (equivalent to anteromedial arm) to anteroposterior width of proximal end (equivalent to anterolateral arm): less than $1.4(0)$; 1.4 to <2.0 (1); 2.0 or greater (2) (Wilson, 2002; Mannion et al., 2013; Upchurch et al., 2015; the long-axes of the anteromedial and anterolateral processes are extrapolated posteriorly so that they intersect close to the position of the olecranon, and each process length is then measured from this intersection to the tip of each process) [ordered]. 
C52. Metacarpals, longest metacarpal to radius proximodistal length ratio: less than $0.40(0)$; 0.40 or greater (1) (Mclntosh, 1990; Calvo \& Salgado, 1995; Upchurch, 1998; Wilson \& Sereno, 1998; Upchurch et al., 2004).

C53. Metacarpals, metacarpal I proximal end dorsoventral height to mediolateral width ratio: less than 1.8 (0); 1.8 or greater (1) (Apesteguía, 2005a; Mannion \& Calvo, 2011; Mannion et al., 2013; note that the metacarpal is measured with the flat surface of the ' $D$ ' shape facing laterally, such that the long axis is dorsoventrally aligned).

C54. Metacarpals, metacarpal I to metacarpal II or III proximodistal length ratio: less than 1.0 (0); 1.0 or greater (1) (Upchurch, 1998; note that an average is taken when both metacarpals II and III are preserved).

C55. Metacarpals, metacarpal I to metacarpal IV proximodistal length ratio: less than $1.0(0)$; 1.0 or greater (1) (Wilson \& Sereno, 1998).

C56. Manual ungual on digit I to metacarpal I proximodistal length ratio: 0.5 or greater (0); less than 0.5 (1) (Upchurch et al., 2004; Mannion et al., 2013; revised here so that taxa that have lost their manual phalanges [derived state for C242] are scored as a "?").

C57. Ilium, pubic peduncle (measured at the articular surface), anteroposterior to mediolateral width ratio: greater than 0.5 (0); 0.5 or less (1) (Taylor, 2009; Mannion et al., 2013).

C58. Pubis, iliac articular surface, anteroposterior to mediolateral width ratio: less than 2.0 (0); 2.0 or greater (1) (Mannion \& Calvo, 2011; Mannion et al., 2013).

C59. Pubis, dorsoventral height of ischial articulation of the pubis divided by pubis proximodistal length is: 0.4 or greater (0); less than 0.4 (1) (Salgado et al., 1997; Wilson \& Sereno, 1998; Upchurch et al., 2004; Mannion et al., 2013).

C60. Ischium to pubis proximodistal length ratio: greater than $0.8(0) ; 0.80$ or less (1) (Calvo \& Salgado, 1995; Salgado et al., 1997; Upchurch, 1998; Mannion et al., 2013).

C61. Ischium, ratio of anteroposterior length of proximal plate to ischium proximodistal length: greater than 0.25 (0); 0.25 or less (1) (Mannion et al., 2013).

C62. Ischium, ratio of anteroposterior length of iliac peduncle to anteroposterior length of proximal plate: less than 0.7 (large ischial contribution to acetabulum) (0); 0.7 or greater (small ischial contribution to acetabulum) (1) (Wilson, 2002; D'Emic, 2012; Mannion et al., 2013).

C63. Ischium, ratio of dorsoventral width across the distal shaft (mediolateral in taxa with fully coplanar shafts) to ischium proximodistal length: 0.2 or greater (0); less than 0.2 (1) (Jacobs et al., 1993; Upchurch, 1998; Upchurch et al., 2004; Mannion et al., 2013).

C64. Ischium, ratio of dorsoventral width of distal end of shaft to minimum shaft dorsoventral width (both dimensions are mediolateral in taxa with fully coplanar shafts): 1.5 or greater (0); less than 1.5 (1) (Berman \& McIntosh, 1978; McIntosh, 1990; Upchurch, 1995, 1998; Mannion et al., 2013).

C65. Femur shaft eccentricity, mediolateral width to anteroposterior width ratio at midshaft: less than 1.85 (0); 1.85 or greater (1) (Wilson, 2002; Mannion et al., 2013).

C66. Femoral distal condyles, tibial to fibular condylar anteroposterior length ratio: less than 1.2 (0); 1.2 or greater (1) (Upchurch et al., 2004; Mannion et al., 2013).

C67. Tibia, distal end mediolateral width to long-axis of a cross section horizontally through the midshaft ratio: 2.0 or greater (0); less than 2.0 (1) (Wilson, 2002; Mannion et al., 2013).

C68. Tibia, distal end, mediolateral to anteroposterior width ratio: 1.5 or greater (0); less than 1.5 (1) (Salgado et al., 1997; Upchurch et al., 2004; Mannion et al., 2013).

C69. Fibula, mediolateral width of distal end to mediolateral width at midshaft ratio: 2.0 or greater (0); less than 2.0 (1) (Wilson, 2002; Mannion et al., 2013). 
C70. Astragalus, mediolateral width to maximum proximodistal height ratio: 1.8 or greater (0); less than 1.8 (1) (Wilson, 2002; Mannion et al., 2013).

C71. Astragalus, mediolateral width to maximum anteroposterior length ratio: 1.5 or greater (0); less than 1.5 (1) (D’Emic, 2012; Mannion et al., 2013).

C72. Metatarsals, metatarsal I to metatarsal V proximodistal length ratio: less than $1.0(0)$; 1.0 or greater (1) (Mannion et al., 2013).

C73. Metatarsals, metatarsal III to tibia proximodistal length ratio: less than $0.25(0) ; 0.25$ or greater (1) (Wilson \& Sereno, 1998; Upchurch et al., 2004; Mannion et al., 2013).

C74. Metatarsals, metatarsal $\mathrm{V}$ proximal end to distal end maximum mediolateral width ratio: 1.6 or greater (0); less than 1.6 (1) (Mannion et al., 2013).

C75. Premaxilla, posterolateral processes and lateral processes of maxilla: without midline contact (0); with midline contact forming marked narial depression, subnarial foramen not visible laterally (1) (Upchurch, 1998; Wilson \& Sereno, 1998).

C76. Premaxillary anterior margin, shape: with step (0); without step (1) (Upchurch, 1995, 1998; Wilson \& Sereno, 1998; Mannion et al., 2013).

C77. Premaxilla-maxilla sutural contact, shape in lateral view: straight (0); sinuous (1) (Chure et al., 2010).

C78. Maxillary ascending process, medial plate-like projections: do not contact each other on the midline (0); contact each other on the midline (1) (Upchurch, 1998).

C79. Maxilla, preantorbital fenestra: absent (0); present (1) (Berman \& Mclntosh, 1978; Upchurch, 1995, 1998; Wilson \& Sereno, 1998).

C80. Lacrimal, anterior process: absent (0); present (1) (Wilson, 2002; polarity reversed here).

C81. Jugal-quadratojugal contact: articulation point includes the posterior margin of jugal (0); posterior margin of jugal excluded from articulation and only the ventral margin of the jugal contributes to articulation (1) (Curry Rogers, 2005; Mannion et al., 2013).

C82. Prefrontal, shape of posterior end in dorsal view: acute, with a subtriangular outline (0); broadly rounded or 'square' (1) (Berman \& McIntosh, 1978; Upchurch, 1998; Mannion et al., 2013).

C83. Frontal, medial convexity in dorsal view: absent (0); present (1) (Curry Rogers, 2005).

C84. Frontal, position of parietal suture relative to supratemporal fenestra: close to anterior margin of the fenestra (0); at approximately the centre of the fenestra or more posteriorly (1) (Tschopp \& Mateus, 2013).

C85. Parietal, contribution to post-temporal fenestra: present (0); absent (1) (Wilson, 2002).

C86. Supratemporal fenestra, lateral exposure: visible laterally, temporal bar shifted ventrally (0); not visible laterally, obscured by temporal bar (1) (Wilson \& Sereno, 1998; Mannion et al., 2013).

C87. Postorbital, ventral process: anteroposterior and mediolateral diameters equal, or mediolaterally compressed (0); anteroposteriorly compressed (1) (Wilson \& Sereno, 1998; Upchurch et al., 2004).

C88. Infratemporal (or laterotemporal) fenestra, anterior extension: reaching midpoint of orbit (0); reaching or surpassing anterior margin of orbit (1) (Upchurch, 1995, 1998; Ksepka \& Norell, 2010; Mannion et al., 2013).

C89. Squamosal-quadratojugal contact: present (0); absent (1) (Gauthier, 1986; Upchurch, 1995, 1998; Wilson \& Sereno, 1998).

C90. Quadratojugal, anterior ramus, ventral triangular projection (close to the anterior tip): absent (0); present (1) (D’Emic, 2012). 
C91. Quadrate, excavation in the posterior surface: absent or shallow (0); deep (1) (Wilson \& Sereno, 1998; Upchurch et al., 2004).

C92. Quadrate fossa, orientation: posterior (0); posterolateral (1) (Wilson, 2002).

C93. Palatobasal contact for basipterygoid articulation has a dorsomedially oriented 'hook'or 'finger'-like projection which curves round to clasp the end of the basipterygoid process: present (0); absent (1) (Madsen et al., 1995; Upchurch, 1998; Wilson \& Sereno, 1998; Wilson, 2002; Mannion et al., 2013).

C94. Palatine, dorsomedial blade (that articulates with maxilla), lateral margin: curved (0); straight (1) (Wilson \& Upchurch, 2009; Mannion et al., 2013).

C95. Vomer, anterior articulation with: maxilla (0); premaxilla (1) (Wilson, 2002).

C96. Paroccipital process, ventral non-articular process: absent (0); present (1) (Wilson, 2002).

C97. Basal tubera, degree of divergence: no divergence, or restricted to ventral half of basal tubera (0); extends into dorsal half of basal tubera, usually fully divergent (1) (Curry Rogers, 2005; Mannion, 2011; Mannion et al., 2013).

C98. Basioccipital, foramen or pit on the posterior surface of the basal tubera: absent (0); present (1) (Wilson, 2002; Mannion, 2011; Mannion et al., 2013; revised here to clarify character statement following Tschopp et al., 2015a).

C99. Basioccipital, foramen/foramina between basal tubera and basipterygoid processes: present (0); absent (1) (Wilson, 2002; Mannion, 2011; Mannion et al., 2013).

C100. Basal tubera, posterior surface bordered laterally and ventrally by a raised, thickened lip: absent (0); present (1) (Wilson, 2002, 2005b; modified based on Mannion, 2011).

C101. Basipterygoid processes, shape in cross-section: elliptical or subtriangular (0); subcircular (1) (Upchurch et al., 2004).

C102. External mandibular fenestra: present (0); absent (1) (Mclntosh, 1990; Upchurch, 1995).

C103. Dentary, posteroventral process, shape: single (0); forked (1) (Chure et al., 2010).

C104. Tooth rows: restricted anterior to orbit (0); restricted anterior to antorbital fenestra (1); restricted anterior to external naris (2); restricted anterior to subnarial foramen (3) (Gauthier, 1986; Upchurch, 1998; Wilson, 2002; Chure et al., 2010; Mannion et al., 2013) [ordered].

C105. Teeth, occlusal (wear) pattern: interlocking, V-shaped facets (0); high angled planar facets (1); low angled planar facets (2) (Wilson \& Sereno, 1998).

C106. Tooth crowns, orientation: aligned anterolingually, tooth crowns overlap (0); aligned along jaw axis, crowns do not overlap (1) (Wilson, 2002; Mannion et al., 2013).

C107. Tooth crowns in upper and lower tooth rows, relative diameters: subequal (0); lower crowns smaller than upper crowns (1) (Chure et al., 2010).

C108. Tooth crowns, shape in labial view: spatulate or 'spoon'-like (i.e. constricted at the base relative to midheight of the crown) (0); parallel-sided (i.e. little expansion above the root) (1) (Calvo, 1994; Upchurch, 1998).

C109. Tooth crowns, cross-sectional shape at mid-crown: 'D'-shaped (0); cylindrical (1) (Wilson \& Sereno, 1998; Mannion et al., 2013).

C110. Tooth crowns, lingual surface: concave or flat (0); convex (1) (Upchurch, 1998; Upchurch et al., 2004; Mannion et al., 2013).

C111. Tooth crowns, apicobasally oriented lingual ridge: present (0); absent (1) (Barrett et al., 2002; Mannion et al., 2013). 
C112. Tooth crowns, distinct mesial and distal carinae (labiolingually thinner than the rest of the tooth crown) along the full crown length: absent (0); present (1) (Mannion, 2011; Mannion et al., 2013).

C113. Tooth serrations/denticles: present (0); absent (1) (Wilson, 2002; Upchurch et al., 2004; Mannion et al., 2013).

C114. Maxillary teeth, shape: straight along axis (0); twisted axially through an arc of 30-45 degrees (1) (Chure et al., 2010; D’Emic, 2012).

C115. Cervical and anteriormost dorsal vertebrae, internal tissue structure: solid (0); camerate (1); camellate (2) (Wilson \& Sereno, 1998; Carballido et al., 2011; Mannion et al., 2013).

C116. Atlantal intercentrum, occipital facet shape: rectangular in lateral view, length of dorsal aspect subequal to that of ventral aspect (0); expanded anteroventrally in lateral view, anteroposterior length of dorsal aspect shorter than that of ventral aspect, producing an anteroventral lip (1) (Wilson, 2002).

C117. Cervical axis, midline ventral keel: absent (0); present (1) (Mannion, 2011; Mannion et al., 2013).

C118. Postaxial cervical centra, anterior half of ventral surfaces are: flat or mildly convex mediolaterally (0); concave mediolaterally (1) (Upchurch, 1998; Mannion et al., 2013).

C119. Postaxial cervical centra, posterior half of ventral surfaces are: flat or mildly convex mediolaterally (0); concave mediolaterally (1) (Upchurch, 1998; Mannion et al., 2013).

C120. Postaxial cervical centra, ventral midline keel: present (0); absent (1) (Upchurch, 1998; Mannion et al., 2013).

C121. Postaxial cervical centra, parapophyses dorsally excavated: absent (0); present (1) (Upchurch, 1998; Mannion et al., 2013).

C122. Postaxial cervical centra, lateral surfaces: lack an excavation or have a shallow fossa (0); possess a deep foramen that is not divided into portions by accessory laminae (1); have a deep foramen that is divided into separate portions by one prominent and occasionally several smaller accessory laminae (2) (McIntosh, 1990; Russell \& Zheng, 1993; Upchurch, 1995, 1998) [ordered].

C123. Middle cervical centra, lateral pneumatic fossa/foramen extends almost to the posterior end of the centrum, leaving only a thin strip of bone: absent (0); present (1) (Wedel et al., 2000; D’Emic, 2013; Mannion et al., 2013).

C124. Middle-posterior cervical centra, parapophyses, dorsal surfaces: face dorsally or slightly dorsolaterally (0); deflected to face strongly dorsolaterally, such that the cervical ribs are displaced ventrally at least the same height as the centrum (1) (Wilson \& Upchurch, 2009; D'Emic, 2012; Mannion et al., 2013).

C125. Middle-posterior cervical centra, parapophyses: restricted to anterior half of centrum (excluding condylar ball) (0); elongate, extending more than half of the centrum length (excluding condylar ball) (D'Emic, 2012; Mannion et al., 2013).

C126. Cervical neural arches, 'pre-epipophyses' present on prezygapophyses: absent (0); present (1) (Wilson \& Upchurch, 2009; Mannion et al., 2013).

C127. Cervical neural arches, epipophyses present on postzygapophyses: absent (0); present (1) (Yates, 2007).

C128. Cervical neural arches (post-Cv3), epipophyses: do not extend beyond the posterior margin of the postzygapophyses (0); extend beyond the posterior margin of the postzygapophyses (usually as prongs) (1) (Sereno et al., 1993; Yates, 2007; D'Emic, 2012; Mannion et al., 2013). 
C129. Cervical neural arches, epipophyseal-prezygapophyseal lamina (EPRL): absent (0); present (1) (Sereno et al., 2007; Wilson \& Upchurch, 2009).

C130. Middle-posterior cervical neural arches, centroprezygapophyseal lamina (CPRL): single (0); bifurcates into medial and lateral branches that both contact the prezygapophysis (1) (Upchurch, 1995, 1998; Wilson, 2002; Whitlock 2011a; Mannion et al., 2013).

C131. Middle-posterior cervical neural arches, intrapostzygapophyseal lamina (TPOL) projects beyond the posterior margin of the neural arch (including the centropostzygapophyseal laminae [CPOL]), forming a prominent subrectangular projection in lateral view: absent (0); present (1) (D’Emic, 2012; Mannion et al., 2013).

C132. Postaxial cervical and anterior dorsal neural spines: unbifurcated (0); bifurcated (1) (Gauthier, 1986; McIntosh, 1990; Upchurch, 1995, 1998; Wilson \& Sereno, 1998).

C133. Cervical bifurcated neural spines (excluding the posteriormost cervical vertebrae), median process at base of 'notch': absent (0); present (1) (Gauthier, 1986; Mclntosh, 1990; Upchurch, 1995, 1998; Wilson \& Sereno, 1998; Mannion et al., 2013).

C134. Middle cervical neural spines, abrupt increase in height (height approximately doubled), following low anterior cervical neural spines (occurs around CV6-8): absent (0); present (1) (Wedel et al., 2000; Rose, 2007; Mannion et al., 2013).

C135. Middle cervical neural spines, dorsal surface with mediolaterally oriented midline ridge flanked by small fossae at its anterior and posterior ends: absent (0); present (1) (D’Emic, 2013; Mannion et al., 2013).

C136. Posterioriormost cervical and anterior dorsal neural arches, spinodiapophyseal lamina (SPDL): single structure (0); divided into anterior and posterior spinodiapophyseal laminae (1) (Salgado et al., 1997; Salgado \& Powell, 2010; D’Emic, 2012).

C137. Posteriormost cervical and anterior dorsal unbifurcated neural spines, prespinal lamina: absent (0); present (1) (Salgado et al., 1997; D’Emic, 2012).

C138. Posteriormost cervical and anterior dorsal bifurcated neural spines, 'trifid' with median tubercle at least as tall as metapophyses: absent (0); present (1) (Wilson \& Upchurch, 2009; D’Emic, 2012; Mannion et al., 2013).

C139. Cervical ribs, longest shafts are: short and do not project far beyond the end of the centrum to which they attach (0); elongate and form overlapping bundles (1) (McIntosh, 1990; Upchurch, 1995, 1998; Mannion et al., 2013).

C140. Cervical ribs, longest shafts extend beneath: fewer than 3 vertebrae (0); 3 vertebrae or more (1) (Wedel et al., 2000; Mannion et al., 2013); revised here so that taxa with short ribs [scored with the plesiomorphic state for C139] are scored as a "?").

C141. Middle-posterior dorsal vertebrae, internal tissue texture: solid (0); camerate (1); camellate (2) (Wilson \& Sereno, 1998; Carballido et al., 2011; Mannion et al., 2013).

C142. Middle-posterior dorsal centra, ventral keel: absent (0); present (1) (Mannion et al., 2012; Poropat et al., 2016).

C143. Dorsal centra, lateral pneumatic foramen: absent (0); present (1) (Upchurch, 1998).

C144. Dorsal centra, lateral pneumatic foramina are: shallow fossae or excavations that do not ramify throughout the centrum (0); deep excavations that ramify throughout the centrum and into the base of the neural arch, often leaving only a thin septum on the midline of the centrum (1) (Upchurch, 1998; Mannion et al., 2013).

C145. Dorsal centra, lateral pneumatic foramina: have margins which are flush with the lateral surface of the centrum (0); are set within a lateral fossa on the lateral surface of the centrum (1) (Bonaparte \& Coria, 1993; Upchurch et al., 2004).

C146. Anterior dorsal centra, lateral pneumatic foramina have: rounded posterior margins (0); acute posterior margins (1) (Upchurch, 1998). 
C147. Middle-posterior dorsal centra, anterior articular face shape: flat or concave (0); mildly convex, with degree of convexity notably reducing along the dorsal sequence (1); strongly convex, with degree of convexity approximately consistent along the dorsal sequence (2) (Salgado et al., 1997; Wilson \& Sereno, 1998; Mannion et al., 2013) [ordered].

C148. Middle-posterior dorsal neural arches, posterior centroparapophyseal lamina (PCPL): absent (0); present as a single lamina (1); two parallel laminae (2) (Upchurch, 1998; Wilson, 2002; D'Emic, 2012; Mannion et al., 2013) [ordered].

C149. Middle-posterior dorsal neural arches, hyposphene-hypantrum system: present (0); absent (1) (Salgado et al., 1997; Upchurch, 1998).

C150. Middle-posterior dorsal neural arches, hyposphene shape: narrow, ventral end subequal to or only slightly wider than dorsal tip (0); wide, triangular shape, with ventral end at least twice width of dorsal tip (1) (Apesteguía, 2005b; Mannion et al., 2013).

C151. Middle-posterior dorsal neural arches, posterior centrodiapophyseal lamina (PCDL): has an unexpanded ventral tip (0); expands and bifurcates towards its ventral tip (1) (Salgado et al., 1997).

C152. Middle-posterior dorsal neural arches, postzygodiapophyseal lamina (PODL): present (0); absent (1) (Salgado et al., 1997; Sanz et al., 1999; Mannion et al., 2013).

C153. Anterior dorsal diapophyses are: directed laterally or slightly upwards (0); directed strongly dorsolaterally at approximately $45^{\circ}$ to the horizontal (1) (Upchurch, 1998; Mannion et al., 2013).

C154. Anterior-middle dorsal diapophyses: short and dorsoventrally tall (0); elongate and dorsoventrally narrow (1) (Janensch, 1950; Taylor, 2009; D'Emic, 2012).

C155. Middle-posterior dorsal diapophyses are: directed strongly dorsolaterally at approximately $45^{\circ}$ to the horizontal (0); directed laterally or slightly upwards (1) (Upchurch, 1998; Mannion et al., 2013).

C156. Middle-posterior dorsal diapophyses, distal end: curves smoothly into the remaining dorsal surface of the process (0); is set off from the remaining dorsal surface by a lip, forming a distinct area (1) (Sanz et al., 1999; Upchurch et al., 2004; D’Emic, 2012).

C157. Posteriormost dorsal diapophyses lie: posterior or posterodorsal to the parapophysis (0); vertically above the parapophysis (1) (Upchurch, 1998).

C158. Dorsal neural spines, height: anterior dorsal neural spines subequal to or dorsoventrally shorter than posterior dorsal neural spines (0); anterior dorsal neural spines dorsoventrally taller than posterior dorsal neural spines (1) (Wilson, 2002; Taylor, 2009; Mannion et al., 2013).

C159. Dorsal neural spines, anteroposterior width: approximately constant along the height of the spine, with subparallel anterior and posterior margins (0); narrows dorsally to form a triangular shape in lateral view, with the base approximately twice the width as the dorsal tip (1) (Taylor, 2009; Mannion et al., 2013).

C160. Anterior dorsal neural spines, orientation of anterior margin in lateral view: inclined anteriorly or vertical (0); posterodorsally inclined (1) (Upchurch et al., 2004; Mannion et al., 2013; revised here to clarify character statement).

C161. Middle dorsal neural spines: unbifurcated (0); bifurcated (dorsal surface excavated transversely) (1) (Mannion et al., 2013).

C162. Middle-posterior dorsal neural spines: tapering or not flaring distally (0); flared distally with triangular aliform processes projecting laterally from the top (formed primarily from the expansion of the spinopostzygapophyseal laminae [SPOLs]) (1) (Upchurch, 1998; Wilson \& Sereno, 1998; Wilson, 2002; Upchurch et al., 2004). 
C163. Middle-posterior dorsal neural spines, with triangular aliform processes: weakly developed aliform processes (0); strongly developed triangular aliform processes so that the lateral tips of these processes extend further laterally than the postzygapophyses (1) (Upchurch, 1998; Wilson \& Sereno, 1998; Wilson, 2002; Upchurch et al., 2004; revised here so that only taxa with aliform processes scored for this character).

C164. Middle-posterior dorsal neural spines, orientation: vertical or slightly posterodorsal (0); strongly posterodorsal, oriented at $45^{\circ}$ to the horizontal or greater (1) (Wilson 2002; Poropat et al., 2016; note that this excludes the posteriormost dorsal neural spines, which usually revert to being dorsally directed).

C165. Middle-posterior dorsal neural spines, spinopostzygapophyseal lamina (SPOL) shape: single (0); divided into medial and lateral branches (1) (Wilson, 1999, 2002).

C166. Middle-posterior dorsal neural spines, spinodiapophyseal lamina (SPDL): absent or restricted to posterior dorsals (0); present on middle and posterior dorsals (1) (Wilson, 2002; Upchurch et al., 2004).

C167. Middle-posterior dorsal neural spines, prespinal and postspinal laminae: form mediolaterally wide surfaces, with little anterior relief, 'infilling' the prespinal and postspinal fossae (0); form distinct mediolaterally narrow ridges or laminae along the midline of the prespinal and postspinal fossae (1) (Mannion et al., 2013).

C168. Middle-posterior dorsal neural spines, midline prespinal lamina (forming distinct ridge) along proximal (lower) half of neural spine: present (0); absent (1) (Salgado et al., 1997; Curry Rogers, 2005; Mannion et al., 2013; note that taxa coded as '0' for C167 are scored as a '?' here).

C169. Middle-posterior dorsal neural spines, postspinal lamina (forming distinct ridge) along proximal (lower) half of neural spine: present (0); absent (1) (Bonaparte, 1986b; Upchurch, 1995, 1998; Curry Rogers, 2005; Mannion et al., 2013; note that taxa coded as '0' for C167 are scored as a '?' here).

C170. Thoracic (dorsal) ribs, pneumatised (with proximal pneumatocoels): absent (0); present (1) (Wilson \& Sereno, 1998).

C171. Anterior thoracic ribs, cross-sectional shape: subcircular (0); plank-like, anteroposterior breadth more than three times mediolateral breadth (1) (Wilson, 2002).

C172. Sacral vertebrae, camellate internal tissue structure: absent (0); present (1) (Mannion et al., 2013).

C173. Sacral centra, lateral pneumatic foramina or very deep depressions: absent (0); present (1) (Upchurch, 1998).

C174. Sacral neural spines, dorsal portions of at least sacral vertebrae 1-4 fused, forming a dorsal 'platform': absent (0); present (1) (Lü et al., 2007; Mannion et al., 2013).

C175. Caudal vertebrae, number: more than 35 (0); 35 or fewer (1) (Wilson 2002; Mannion et al., 2013).

C176. Anteriormost caudal vertebrae, camellate internal tissue structure: absent (0); present (1) (Powell, 1986; Wilson, 2002).

C177. Anterior caudal centra with posterior convexity: convex in anteriormost caudal vertebrae, changing to flat or concave in more distal anterior caudal vertebrae (0); convex throughout all anterior caudal vertebrae with ribs (1) (Mannion et al., 2013; revised here to only include taxa with some procoely in the anterior caudal sequence - taxa lacking procoelous anterior caudal vertebrae are scored as a "?").

C178. Anterior caudal centra, lateral pneumatic fossae or foramina: absent (0); present, (McIntosh, 1990; Upchurch, 1995). 
C179. Anterior caudal centra, lateral pneumatic fossae or foramina: without sharply defined margins (0); with sharply defined margins (1) (Tidwell et al., 2001; Whitlock et al., 2011; Mannion et al., 2013).

C180. Anterior-middle caudal centra, small, shallow vascular foramina pierce the lateral and/or ventral surfaces: absent (0); present (1) (Mannion \& Calvo, 2011; Mannion et al., 2013).

C181. Anterior caudal centra (excluding the anteriormost caudal vertebrae), ventral longitudinal hollow: absent (0); present (1) (McIntosh, 1990; Upchurch, 1995, 1998; Wilson, 2002; revised here to separate anterior and middle caudal vertebrae).

C182. Anterior caudal centra (excluding the anteriormost caudal vertebrae), distinct ventrolateral ridges, extending the full length of the centrum: absent (0); present (1) (McIntosh, 1990; Upchurch, 1995, 1998; Upchurch et al., 2004; Mannion et al., 2013; revised here to separate anterior and middle caudal vertebrae).

C183. Middle caudal centra, anteroposteriorly elongate ridge situated at approximately twothirds of the way up the lateral surface: absent (0); present (1) (Upchurch \& Martin, 2003; Mannion et al., 2013).

C184. Middle-posterior caudal centra (at least some), posterior articular surface: flat or concave (0); convex (1) (Jacobs et al., 1993; Upchurch, 1995, 1998; Salgado et al., 1997; Wilson, 2002).

C185. Middle-posterior caudal centra with convex posterior articular surface: condylar convexity merges smoothly into the lateral surface of the main body of the centrum (0); distinct rim rings the condyle, separating it from the lateral surface of the main body of the centrum (1) (Mannion et al., 2013).

C186. Distal caudal centra, biconvex: absent (0); present (1) (Wilson \& Sereno, 1998).

C187. Anterior caudal neural arches, hyposphenal ridge: present (0); absent (1) (Upchurch, 1998; Mannion et al., 2013; usually only present in the anteriormost region of the tail).

C188. Anterior caudal neural arches, hyposphenal ridge shape: slender ridge (0); block-like hyposphene (1) (Taylor, 2009; Mannion et al., 2013).

C189. Anterior caudal neural arches, distinct prezygodiapophyseal lamina (PRDL): absent (0); present (1) (Chure et al., 2010; usually only present in the anteriormost region of the tail).

C190. Anterior caudal neural arches, sharp lipped lateral coel (postzygapophyseal centrodiapophyseal fossa [POCDF] of Wilson et al., 2011) bounded by posterior centrodiapophyseal lamina (PCDL [or caudal rib itself]), centropostzygapophyseal lamina (CPOL) and postzygodiapophyseal lamina (PODL): absent (0); present (1) (Lü et al., 2008; Whitlock et al., 2011; Mannion et al., 2013).

C191. Anterior-middle caudal neural arches, prezygapophyses switch from projecting anterodorsally, anteriorly and back to anterodorsally along the sequence: absent (0); present (1) (Mannion \& Calvo, 2011; Mannion et al., 2013).

C192. Middle caudal neural arches: situated over the mid-point of the centrum with approximately subequal amounts of the centrum exposed at either end (0); located on the anterior half of the centrum (1) (Calvo \& Salgado, 1995; Upchurch, 1995, 1998; Salgado et al., 1997).

C193. Middle-posterior caudal neural arches, distance that prezygapophyses extend beyond the anterior margin of the centrum: less than $20 \%$ of centrum length (excluding ball), short prezygapophyses (0); $20 \%$ or greater of centrum length (excluding ball), elongate prezygapophyses (1) (González Riga, 2003; Mannion et al., 2013; note that in taxa without anteriorly biased neural arches this prezygapophyseal extension is extrapolated as if the arch was anteriorly positioned on the centrum). 
C194. Anteriormost caudal neural spines, sharp lipped lateral coel (spinodiapophyseal fossa [SDF] of Wilson et al., 2011) bounded by spinoprezygapophyseal lamina (SPRL), spinopostzygapophyseal lamina (SPOL) and postzygodiapophyseal lamina (PODL): absent (0); present (1) (Wilson, 2002; Whitlock et al., 2011; Wilson et al., 2011; Mannion et al., 2013).

C195. Anterior caudal neural spines, project: posterodorsally (0); dorsally (sometimes with a subtle anterior deflection) (1); anterodorsally, such that the anterodorsal margin of the neural spine projects beyond the anterior margin of the centrum (2) (Gonzalez Riga et al., 2009; Mannion et al., 2013; Poropat et al., 2016) [ordered].

C196. Anterior caudal neural spines, anterodorsal margin of neural spine: level with or posterior to posterior margin of postzygapophyses (0); situated anterior to posterior margin of postzygapophyses (usually does not even approach postzygapophyses) (1) (Salgado et al., 1997; Mannion et al., 2013).

C197. Anterior caudal neural spines, prespinal and postspinal laminae: absent or form mediolaterally wide surfaces, with little anterior relief, 'infilling' the prespinal and postspinal fossae (0); form distinct mediolaterally narrow ridges or laminae along the midline of the prespinal and postspinal fossae (1) (Mannion et al., 2013).

C198. Anterior caudal neural spines, spinoprezygapophyseal lamina (SPRL)spinopostzygapophyseal lamina (SPOL) contact: absent (0); present, forming a prominent lateral lamina on the neural spine (1) (Wilson, 1999, 2002; revised here so that only taxa in which the SPRL extends onto the lateral surface are scored).

C199. Middle caudal neural spines, in lateral view, widen anteroposteriorly (approximately doubling) from their base to their summit: absent (0); present (1) (Mannion et al., 2013).

C200. Middle caudal neural spines, extend posteriorly to the mid-point (or beyond) of the proceeding caudal centrum: present (0); absent (usually do not extend beyond the posterior margin of the centrum) (1) (Remes et al., 2009; Mannion et al., 2013; note that in taxa with anteriorly biased neural arches this posterior extension is extrapolated as if the arch was centrally positioned on the centrum).

C201. Caudal ribs: present beyond approximately Cd10 (usually at least up to approximately Cd15) (0); only present through to approximately Cd10 (1) (Upchurch, 1998; Wilson, 2002).

C202. First caudal rib (transverse process), with prominent ventral bulge: absent (0); present (1) (Wilson, 2002; Chure et al., 2010; note that this feature is sometimes present in subsequent caudal vertebrae too: consequently, this character is coded as the derived state if present in any anterior caudal vertebrae, but coded as a '?' if the first caudal vertebra is not preserved and the feature is absent in other anterior caudal vertebrae).

C203. First caudal rib, expands anteroposteriorly towards its distal end, forming an 'anchor' shape in dorsal view: absent (0); present (1) (Suteethorn et al., 2010; Mannion et al., 2013). C204. Anterior caudal ribs, shape in anterior view: triangular, tapering distally (0); wing-like, with a dorsolaterally oriented dorsal margin (1) (Berman \& Mclntosh, 1978; Mclntosh, 1990; Upchurch, 1995, 1998; Whitlock et al., 2011).

C205. Anterior caudal ribs: curve strongly anterolaterally (0); mainly laterally (1); curve strongly posterolaterally (2) (Mannion \& Calvo, 2011; D’Emic, 2012; Mannion et al., 2013) [ordered].

C206. Anterior caudal ribs: do not extend beyond posterior end of centrum (excluding posterior ball) (0); extend beyond posterior end of centrum (excluding posterior ball) (1) (Mannion \& Calvo, 2011; Mannion et al., 2013; revised here so that only taxa with posterolaterally directed caudal ribs are scored for this character). 
C207. First chevron, morphology: Y-shaped and does not differ notably from subsequent chevrons (0); anteroposteriorly flattened and V-shaped, with dorsoventrally reduced distal blade (1) (Gomani, 2005; Rauhut, 2006; Mannion \& Calvo, 2011; Mannion et al., 2013).

C208. Anterior chevrons, proximal ends: open dorsally (0); bridged dorsally by a bar of bone (1) (Powell, 1992; Calvo \& Salgado, 1995; Upchurch, 1995, 1998; Mannion et al., 2013).

C209. Anterior-middle chevrons, lateral bulges close to distal ends of chevron blades: absent (0); present (1) (Mannion et al., 2013).

C210. Middle-posterior chevrons, with anterior expansion of distal blade: present (0); absent (1) (Berman \& Mclntosh, 1978; Upchurch, 1995, 1998; Wilson \& Sereno, 1998; Mannion et al., 2013).

C211. Middle-posterior chevrons, with posterior expansion of distal blade (excluding the natural posteroventral curvature of many chevrons): present (0); absent (1) (Berman \& McIntosh, 1978; Upchurch, 1995, 1998; Wilson \& Sereno, 1998; Mannion et al., 2013).

C212. Scapular acromion (proximal plate), area situated posterior to the acromial ridge: flat or convex (0); forms a separate excavated area (1) (Upchurch et al., 2004).

C213. Scapular glenoid surface, orientation: faces anteroventrally and/or slightly laterally (0); deflected to face anteroventrally and medially (1) (Wilson \& Sereno, 1998; Upchurch et al., 2004).

C214. Scapula, posterior margin of the dorsal part of the acromion: straight and oriented vertically, or sloping to face posterodorsally (0); concave, posterodorsal corner of acromion overhangs the dorsal surface of the scapular blade (1) (Rauhut et al., 2005; Mannion, 2009; Mannion et al., 2013).

C215. Scapular acromion, subtriangular process at posteroventral corner: absent (0); present (1) (Smith et al., 2001; Bonaparte et al., 2006; Carballido et al., 2011; D'Emic et al., 2011; Mannion et al., 2013).

C216. Scapular blade, subtriangular process at anteroventral corner: absent (0); present (1) (Carballido et al., 2011; D'Emic et al., 2011; Mannion et al., 2013).

C217. Scapular blade, cross-sectional shape at base: rectangular (0); D-shaped (lateral surface is strongly convex dorsoventrally and medial surface flat) (1) (Wilson, 2002).

C218. Coracoid, anterior and dorsal margins in lateral view: merge smoothly into each other, giving a rounded profile (0); meet each other at an abrupt angle, making the coracoid quadrangular in outline (1) (Upchurch, 1998).

C219. Coracoid, dorsal margin in lateral view: lies below the level of the scapular acromion plate (separated from the latter by a V-shaped notch) (0); reaches or surpasses the level of the dorsal margin of the scapular acromion plate (1) (Upchurch, 1995, 1998; Upchurch et al., 2004; Mannion et al., 2013).

C220. Coracoid, ventral margin in lateral view forms a notch anterior to the glenoid, resulting in an 'infraglenoid lip' anterior to the notch: absent (0); present (1) (Wilson, 2002; Mannion et al., 2013).

C221. Sternal plate, shape in dorsal view: subcircular or oval (0); triangular (created by an acute anterolateral projection) (1); elliptical with a mildly or strongly concave lateral margin (2) (Calvo \& Salgado, 1995; Upchurch, 1998; Wilson, 2002; Upchurch et al., 2004).

C222. Sternal plate, prominent posterolateral expansion produces a 'kidney'-shaped profile in dorsal view: absent (0); present (1) (McIntosh, 1990; Upchurch, 1998).

C223. Humeral proximolateral corner, shape: rounded, surfaces merge smoothly into each other to produce a transversely rounded proximal end, with the proximalmost point of the lateral margin at a lower level than the remaining lateral half of the proximal surface (0); square, surfaces meet each other at an abrupt angle to produce a 'squared' proximal end in 
anterior view, with the proximalmost point of the lateral margin level with the remaining lateral half of the proximal surface (1) (Upchurch, 1999; Wilson, 2002; Upchurch et al., 2004; Harris, 2006b; Mannion et al., 2013).

C224. Humerus, shape of lateral margin of diaphysis (approximately the middle third of the humerus) in anterior view: concave (0); straight (1) (Curry Rogers, 2005; Mannion et al., 2013).

C225. Humeral deltopectoral crest, projection: anteriorly or anterolaterally (0); anteromedially, extending across the anterior face of the humerus (1) (Mannion et al., 2013; Upchurch et al., 2015; Poropat et al., 2016).

C226. Humerus, strong bulge or tuberosity (site for $\mathrm{M}$. latissimus dorsi) close to the lateral margin of the posterior surface, at approximately the level of the distal tip of the deltopectoral crest: absent (0); present (1) (Borsuk-Białynicka, 1977; Otero, 2010; D'Emic, 2012).

C227. Humerus, anterior surface of distal lateral condyle: divided by a notch, forming two ridges (0); undivided (1) (D’Emic, 2012).

C228. Humerus, distalmost part of the posterior surface (supracondylar fossa) is: flat or shallowly concave (0); deeply concave between prominent lateral and medial vertical condylar ridges (1) (Upchurch et al., 2004).

C229. Humeral distal condyles, articular surface: flat anteroposteriorly and restricted to distal portion of humerus (0); anteroposteriorly convex so that it curves up onto the anterior and posterior faces of the humerus (1) (Wilson, 2002; Upchurch et al., 2004).

C230. Humeral distal articular surface, condyles: undivided (0); divided (1) (Wilson, 2002; Mannion et al., 2013).

C231. Radius, strong twist in axis, such that the long-axes of the proximal and distal ends are not oriented in the same plane: absent (0); present (1) (Mannion et al., 2013).

C232. Radius, well developed interosseous ridge that extends along most of the radius length (at least along the distal two-thirds): absent (0); present (1) (Curry Rogers, 2005; Mannion et al., 2013).

C233. Ulnar olecranon process, development: absent or only rudimentary, i.e. projecting just above the proximal articulation (0); prominent, projecting well above proximal articulation (1) (McIntosh, 1990; Wilson \& Sereno, 1998; Mannion et al., 2013).

C234. Ulna, articular surface of anteromedial process is: flat (0); concave along its length (1) (Upchurch, 1995, 1998).

C235. Ulna, orientation of anteromedial process: flat or sloping downwards less than $40^{\circ}(0)$; sloping downwards at an angle of at least $40^{\circ}$ to the horizontal (1) (Mannion et al., 2013).

C236. Ulna, distal end: prominently expanded posteriorly (0); unexpanded (1) (D'Emic, 2012).

C237. Carpal bones, number: 3 or more (0); fewer than 3 (1) (Upchurch, 1995, 1998).

C238. Carpal bones: at least one carpal present (0); absent (1) (Upchurch, 1995, 1998).

C239. Metacarpals, distal articular surfaces: extend onto dorsal/anterior surface of metacarpal (0); restricted to distal surface (except sometimes in metacarpal IV) (1) (Salgado et al., 1997; D’Emic, 2012).

C240. Metacarpals, metacarpal I distal end mediolateral axis orientation: approximately perpendicular (or only gently bevelled) to long axis of shaft (0); bevelled approximately $20^{\circ}$ proximodistally with respect to axis of shaft (1) (Wilson, 2002; Mannion et al., 2013).

C241. Metacarpals, metacarpal IV has a prominent proximolateral projection that wraps around the dorsal (anterior) face of metacarpal $V$ (metacarpal IV often forms a chevron shape in proximal end view): absent (0); present (1) (D'Emic, 2012; Mannion et al., 2013). 
C242. Manual digits: possess at least some phalanges (0); have lost the phalanges (1) (Wilson, 2002; Upchurch et al., 2004).

C243. Manual phalanx I.1, shape in dorsal view: rectangular (0); wedge-shaped (1) (Wilson, 2002).

C244. Ilium, preacetabular process in dorsal view: projects anteriorly (0); projects anterolaterally (1) (Upchurch et al., 2004).

C245. Ilium, preacetabular process orientation: lies in an approximately vertical plane (0); turns laterally towards its ventral tip to form a horizontal portion (1) (Mclntosh, 1990; Powell, 1992; Upchurch, 1995, 1998).

C246. Ilium, preacetabular process shape: dorsoventrally tapers anteriorly to a point (0); semicircular, or rounded outline, such that it does not continue to taper along its anteriormost portion (1) (Calvo \& Salgado, 1995; Upchurch, 1998; Wilson \& Sereno, 1998; Mannion et al., 2013).

C247. Ilium, preacetabular process, bulge or 'kink' on ventral margin: absent (0); present (1) (D’Emic, 2012).

C248. Ilium, highest point on the dorsal margin: occurs level with or posterior to the anterior margin of the base of the pubic process (0); occurs anterior to the anterior margin of the base of the pubic process (1) (Upchurch, 1998; Mannion et al., 2013).

C249. Ilium, pneumatised: absent (0); present (1) (Wilson \& Upchurch, 2009; Mannion et al., 2013).

C250. Pubis, obturator foramen, in lateral view is: subcircular (0); oval or elliptical, with long axis oriented in same plane as long axis of pubis (1) (Mannion \& Calvo, 2011; Mannion et al., 2013).

C251. Pubis, anterior margin of distal end strongly concave in lateral view, such that the distal end forms a prominent, anteriorly expanded boot: absent (0); present (1) (Naish \& Martill, 2001; Mannion et al., 2013).

C252. Ischium, acetabular margin, in lateral view: flat or mildly concave (0); strongly concave, such that the pubic articular surface forms an anterodorsal projection (1) (D'Emic, 2012; Mannion et al., 2013).

C253. Ischium, symphysis between the ischia: terminates at the base of the proximal plates (emarginate distal to pubic articulation) (0); extends along the ventral edges of the proximal plates as well as the distal shafts, so that there is no V-shaped gap between the anterior ends of the ischia in dorsal view (no emargination distal to pubic articulation) (1) (McIntosh, 1990; Upchurch, 1998; Wilson, 2002).

C254. Ischium, long-axis of shaft, if projected upwards/proximally: passes through the lower part of the acetabular margin or the upper part of the pubic articular surface (i.e. it is approximately $60^{\circ}$ to the horizontal in lateral view) (0); passes through the upper part of the acetabular margin or even approaches the rim of the iliac articulation (i.e. the shaft is at approximately $80^{\circ}$ to the horizontal) (1); passes through the lower part of the pubic articular surface (i.e. it is approximately horizontally oriented), such that the posterior margin of the iliac peduncle and the dorsal margin of the shaft form a right angle in lateral view (2) (Upchurch, 1995, 1998; Wilson and Sereno, 1998; revised here to add an extra state) [unordered].

C255. Femur, proximolateral margin, above the lateral bulge: level with or lateral to the lateral margin of the distal half of the shaft (0); medial to the lateral margin of the distal half of the shaft (1) (Mclntosh, 1990; Calvo \& Salgado, 1995; Salgado et al., 1997; RoyoTorres, 2009; Royo-Torres et al., 2012; Mannion et al., 2013). 
C256. Femur, anteroposterior thickness of lateral margin of proximal third: relatively constant with main body of femur (0); narrows to form a flange-like trochanteric shelf, forming a medially bounding vertical ridge along the posterior surface (1) (Mannion et al., 2013).

C257. Femur, proximodistally elongate midline ridge (linea intermuscularis cranialis) on anterior face, extending along most of shaft length: absent (0); present (1) (Otero, 2010; D’Emic, 2012).

C258. Femur, fourth trochanter: not visible in anterior view (0); visible in anterior view (1) (Gallina \& Apesteguía, 2005; Whitlock, 2011a).

C259. Femoral distal condyles, orientation relative to long axis of femoral shaft: bevelled dorsolaterally approximately $10^{\circ}$ (tibial condyle extends further distally than fibular condyle) (0); perpendicular (tibial and fibular condyles extend approximately the same distance distally) (1); bevelled dorsomedially approximately $10^{\circ}$ (fibular condyle extends further distally than tibial condyle) (2) (Wilson, 2002; Mannion et al., 2013) [ordered].

C260. Tibia, cnemial crest projects: laterally (0); anteriorly or anterolaterally (1) (Wilson \& Sereno, 1998; Mannion et al., 2013; note that the tibia is oriented so that the flat, mediolaterally wide triangular surface of the distal end faces anteriorly).

C261. Tibia, lateral edge of proximal end forms a pinched out projection, posterior to cnemial crest (the "second cnemial crest" of Bonaparte et al., 2000): present (0); absent (1) (Bonaparte et al., 2000; Mannion et al., 2013).

C262. Fibula, proximal end with anteromedialy directed crest extending into a notch behind the cnemial crest of the tibia: absent (0); present (1) (Wilson \& Upchurch, 2009; D'Emic, 2012; Mannion et al., 2013).

C263. Fibula, lateral muscle scar is: oval in outline (0); formed from two vertically elongate, parallel ridges (1) (Powell, 1992; Upchurch, 1998).

C264. Fibula, shaft in lateral view: straight (0); sigmoidal (1) (Canudo et al., 2008; RoyoTorres, 2009).

C265. Astragalus, in dorsal (or proximal) view: rectangular, with anteroposterior lengths of medial and lateral margins subequal (or medial margin greater) (0); wedge-shaped, narrowing anteroposteriorly towards its medial end, such that it has a reduced anteromedial corner (1) (Cooper, 1981; Upchurch, 1995, 1998; Wilson \& Sereno, 1998).

C266. Astragalus, ascending process: limited to anterior two-thirds of astragalus (0); extends beyond anterior two-thirds of astragalus (usually to the posterior margin) (1) (Wilson \& Sereno, 1998; Wilson, 2002; revised here following Tschopp et al., 2015a).

C267. Astragalus, laterally-directed ventral shelf underlies the distal end of the fibula: present (0); absent (1) (Wilson \& Upchurch, 2009; Mannion et al., 2013).

C268. Astragalus: caps most, or all, of the distal end of the tibia (0); reduced so that medial edge of tibia is uncapped (1) (Wilson \& Upchurch, 2009; Ksepka \& Norell, 2010).

C269. Astragalus, posterior margin bears a tongue-like projection posteromedial to the ascending process, which is separated from the latter by a groove: present (0); absent (1) (D'Emic, 2012; Mannion et al., 2013).

C270. Calcaneum: present (0); absent (1) (Mclntosh, 1990; Upchurch, 1995, 1998).

C271. Calcaneum, shape in proximal view: subcircular (0); subrectangular (1) (Wilson \& Upchurch, 2009; Mannion et al., 2013).

C272. Metatarsals, metatarsal I with a prominent ventrolateral expansion along its distal half, such that the distal end expands further laterally than the proximal end: absent (0); present (1) (Berman \& McIntosh, 1978; Mclntosh, 1990; Upchurch, 1995, 1998; D’Emic et al., 2011; Mannion et al., 2013). 
C273. Metatarsals, lateral margin of metatarsal II in proximal view: concave (0); straight (1) (Mannion et al., 2013).

C274. Metatarsals, metatarsal II distal articular surface extends up on to the dorsal surface (extending proximally approximately $25 \%$ of metatarsal length and most prominently along medial half): absent (0); present (1) (D'Emic et al., 2011; Mannion et al., 2013).

C275. Metatarsals, medial surface of the proximal portion of metatarsal IV concave (for reception of metatarsal III): absent (0); present (1) (D'Emic et al., 2011; D'Emic, 2012).

C276. Metatarsals, distal end orientation of metatarsal IV: perpendicular to long axis of bone (0); bevelled to face medially (1) (D'Emic, 2012).

C277. Pedal digit IV: has at least three phalanges (0); has two phalanges or fewer (1) (Upchurch, 1995, 1998; Upchurch et al., 2004).

C278. Pedal unguals, tuberosity on the ventral margin, along distal half: absent (0); present (1) (Canudo et al., 2008; Mannion et al., 2013).

C279. Osteoderms: absent (0); present (1) (Wilson, 2002; note that taxa are only coded as the plesiomorphic state when osteoderms are not found associated with a relatively complete postcranial skeleton or a specimen preserving numerous axial elements).

C280. Humerus, strong bulge or tuberosity (site for $M$. scapulohumeralis anterior) on the lateral margin of the posterior surface (usually visible in anterior view), approximately level with the most prominently developed portion of the deltopectoral crest: absent or weakly developed, and not visible in anterior view (0); present, forms a distinct lateral bulge that interrupts the line of the lateral humeral margin in anterior view (1) (Borsuk-Białynicka, 1977; Upchurch et al., 2015).

C281. Ulna, posterior process of proximal end: weakly developed, so that the proximal profile of the ulna is ' $\mathrm{V}$ '-shaped (formed by the anteromedial and anterolateral processes) (0); strongly developed, so that the proximal profile of the ulna is ' $T$ '- or ' $Y$ '-shaped, and there is a deep fossa between the anteromedial and posterior processes, rivalling the radial fossa in depth (1) (Upchurch et al., 2015).

C282. Ulna, shape of the distal end: comma-shaped, with tapering curved anterior process associated with an anteromedial fossa for reception of the radius (0); elliptical or oval in outline, with the anteromedial fossa strongly reduced or absent (1) (Upchurch et al., 2015).

C283. Radius, profile of proximal end: ' $D$ '-shaped or elliptical (0); oval or subtriangular, with marked tapering towards the medial process (1) (Upchurch et al., 2015).

C284. Radius, ridge or flange on medial margin, near proximal end, for attachment of the $M$. biceps brachii and M. brachialis inferior: absent or very weakly developed (0); present, projecting beyond the medial margin of the main radial shaft (1) (Borsuk-Białynicka, 1977; Upchurch et al., 2015).

C285. Radius, posterior margin of distal end: lacks condylar-like processes and fossa (0); forms two low rounded processes (posteromedial and posterolateral), with a shallow fossa between them (1) (D'Emic, 2013; Upchurch et al., 2015).

C286. Snout, shape in dorsal view, Premaxillary-Maxillary Index (PMI): $60 \%$ or less (0); $>60 \%$ to 75\% (1); greater than 75\% (2) (Upchurch, 1998; Whitlock, 2011a; Poropat et al., 2016; note that this can be calculated using the lower jaw when the skull is incomplete) [ordered].

C287. Premaxilla, shape of ascending process in lateral view: convex (0); concave, with a large dorsal projection (1); sub-rectilinear and directed posterodorsally (2) (Whitlock, 2011a).

C288. Maxilla, anterior (dentigerous) portion of lateral surface excavated by dorsoventrally elongate, deep vascular grooves: absent (0); present (1) (Wilson, 2002, 2005b). 
C289. Maxilla, foramen anterior to the preantorbital fenestra: absent (0); present (1) (Zaher et al., 2011).

C290. Maxilla, position of external opening of the preantorbital fenestra: lies below antorbital fenestra (0); lies anterior to antorbital fenstra, with the posterior margin of the preantorbital fenestra lying entirely anterior to the anterior margin of the antorbital fenestra (1) (Marpmann et al., 2015; Poropat et al., 2016).

C291. Maxilla, preantorbital fenestra development: weakly developed, shallow fossa (difficult to distinguish from posterior maxillary foramen) (0); deep, sharp-lipped fossa (1) (Zaher et al., 2011; Carballido et al., 2012; Poropat et al., 2016).

C292. Maxilla, posterior extent of dorsal (ascending) process: anterior to, or level with, posterior end of main body (0); extending posterior to posterior end of main body (1) (Whitlock, 2011a).

C293. Maxilla, ventral margin of jugal process: reduced in dorsoventral height, such that the ventral margin is strongly emarginated relative to the remainder of the ventral margin of the maxilla (0); continuous with ventral margin of remainder of maxilla, or very gently emarginated (1) (Curry Rogers, 2005; Poropat et al., 2016).

C294. Maxilla, contact with quadratojugal: absent or small (i.e. no more than a point contact) (0); extensive (1) (Upchurch, 1995; Poropat et al., 2016).

C295. Jugal, dorsal process: present (0); absent (1) (Tschopp et al., 2015a).

C296. Jugal, contact with ectopterygoid: present (0); absent, ectopterygoid contacts maxilla instead (1) (Upchurch, 1995).

C297. Frontal, anteroposterior length to transverse width ratio: 1.0 or greater (0); less than 1.0 and $>0.5$ (1); 0.5 or less (2) (Whitlock, 2011a; Tschopp \& Mateus, 2013; Poropat et al., 2016; revised here to add an extra state) [ordered].

C298. Frontal, lateral margin: expands posteriorly, orbital margin concave in dorsal view (0); unexpanded posteriorly, orbital margin straight or convex in dorsal view (1) (Whitlock et al., 2010; Poropat et al., 2016).

C299. Frontal, contribution to margin of supratemporal fenestra: present (0); absent, frontal excluded from anterior margin of fenestra by a postorbital-parietal contact (1) (Wilson \& Sereno, 1998).

C300. External nares, position: retracted to level of orbit, facing laterally (0); retracted to position between orbits, facing dorsally or dorsolaterally (1) (McIntosh, 1990; Upchurch, 1995; Whitlock, 2011a).

C301. Lateral temporal fenestra, shape in lateral view: taller than wide anteroposteriorly and subtriangular (anteroposteriorly broader ventral margin and narrower dorsal apex) (0); linear, slit-like, crescentic (longer anteroposteriorly than high dorsoventrally) (1) (Harris, 2006b; Poropat et al., 2016).

C302. Postorbital, posterior (squamosal) process: present as a distinct process (0); absent (1) (Wilson, 2002, 2005b).

C303. Parietal, relative height of suture with frontal: lies level with or above the dorsal surfaces of the frontals and parietals (0); lies below the dorsal surfaces of the parietals and frontals (i.e. in a deep transverse trough) (1) (Curry Rogers, 2005; Poropat et al., 2016).

C304. Parietal, elevation of anterior margin creates a step-like curving crest transversely, where the parietal meets the frontal: absent (0); present (1) (Curry Rogers \& Forster, 2004; Curry Rogers, 2005; Poropat et al., 2016).

C305. Pterygoid, morphology: robust element (0); plate-like, with its three processes coplanar (1) (Curry Rogers \& Forster, 2004; Wilson, 2005a; Poropat et al., 2016). 
C306. Pterygoid, sutural contact with ectopterygoid: anteroposteriorly elongate, along the medial or lateral surface (0); anteroposteriorly short, restricted to the anterior tip of the pterygoid (1) (Zaher et al., 2011; Poropat et al., 2016).

C307. Pterygoid, palatobasal contact for basipterygoid articulation with a convex, rocker-like articular surface: absent (0); present (1) (Wilson, 2002).

C308. Supraoccipital, longitudinal groove along posterodorsal surface: absent (0); present (i.e. sagittal crest divided into two subparallel parasagittal crests with central groove) (1) (Curry Rogers \& Forster, 2004; Curry Rogers, 2005; González Riga et al., 2009).

C309. Supraoccipital-exoccipital-opisthotic, paired facets for articulation with the proatlas: absent (0); present (1) (Poropat et al., 2016).

C310. Parasphenoid rostrum, cross-sectional shape: triangular (0); transversely thin, sheetlike (1) (Berman \& Mclntosh, 1978; Upchurch, 1995, 1998; Tschopp et al., 2015a; Poropat et al., 2016)

C311. Basipterygoid processes, shape: widely diverging at $30^{\circ}$ or more (0); narrowly diverging at less than $30^{\circ}$ (1) (Upchurch, 1995, 1998; Mannion et al., 2012).

C312. Basipterygoid processes, orientation in lateral view: directed $80^{\circ}$ or more to skull roof (normally perpendicular) (0); angled less than $80^{\circ}$ to skull roof (anteroventrally directed) (1) (Mclntosh, 1990; Wilson, 2002; Whitlock, 2011a; Mannion et al., 2012; Poropat et al., 2016).

C313. Basioccipital, orientation of occipital condyle relative to the horizontal plane (in lateral view with supraoccipital held in a vertical plane): $60^{\circ}$ or less (0); greater than $60^{\circ}$ (typically close to $90^{\circ}$ ) (1) (Upchurch, 1995, 1998).

C314. Basal tubera, angle of divergence in posterior view: less than $50^{\circ}(0)$; more than or equal to $50^{\circ}$ (1) (Curry Rogers, 2005; Poropat et al., 2016).

C315. Basisphenoid, relative position of the external opening for cranial nerve VI: lies ventral, and generally close, to the opening for cranial nerve III (0); lies anteroventral to, and more distant from, the opening for cranial nerve III (1) (Remes, 2009; Poropat et al., 2016).

C316. Basisphenoid, opening for cranial nerve VI: penetrates the pituitary fossa (0); does not penetrate the pituitary fossa (1) (Paulina Carabajal, 2012; Poropat et al., 2016).

C317. Basioccipital+exoccipital-opisthotic, number of exits for cranial nerve XII: 2 (0); 1 (1) (Paulina Carabajal, 2012; Poropat et al., 2016).

C318. Basisphenoid, position of the external foramen of the internal carotid artery: lateral to basipterygoid process (0); medial to basipterygoid process (1) (Paulina Carabajal, 2012; Poropat et al., 2016).

C319. Dentary, angle between the long-axis of the anterior margin (mandibular symphysis) and the long-axis of the main body of the dentary, in lateral view: greater than $90^{\circ}$, with the dorsal margin of the dentary extending further anteriorly than the ventral margin (0); approximately $90^{\circ}$, with the dorsal and ventral margins extending an equal distance anteriorly (1) (Salgado \& Calvo, 1997; Upchurch, 1998; Wilson, 2002; Poropat et al., 2016).

C320. Tooth crowns, longitudinal groove paralleling mesial and distal margins on the labial surface: labial grooves present (0); absent (1) (Upchurch, 1995; Poropat et al., 2016).

C321. Atlantal intercentrum, ventral margin of posterior surface: straight or convex (0); concave, forming ventrolateral projections (1) (González Riga \& Ortíz David, 2014; Poropat et al., 2016).

C322. Axis, aEl (average elongation index: anteroposterior length of centrum (excluding articular ball if present) divided by mean average value of the mediolateral width and 
dorsoventral height of posterior articular surface of centrum): 2.0 or greater (0); less than 2.0 (1) (Upchurch et al., 2007; Poropat et al., 2016).

C323. Postaxial cervical centra, pneumatization of lateral surface: lateral pneumatic opening occupies approximately anterior two-thirds of centrum or more (0); reduced and restricted to less than the anterior two-thirds of the centrum (1) (Whitlock, 2011a; Poropat et al., 2016).

C324. Postaxial cervical centra, midline notch on the dorsal margin of the posterior articular surface: absent (0); present (1) (Carballido et al., 2012).

C325. Postaxial anterior cervical vertebrae, prezygapophyses: extend anterior to the anterior tip of the condyle (0); terminate level with or posterior to the anterior tip of the condyle (1) (Curry Rogers, 2005, 2009; Poropat et al., 2016).

C326. Postaxial anterior cervical neural spines, orientation of posterior margin in lateral view: dorsal (vertical) or anterodorsal (0); posterodorsal (1) (Curry Rogers, 2005).

C327. Middle cervical neural spines, height to arch height ratio: 2.0 or lower (0); greater than 2.0 (1) (Rauhut et al., 2005; Whitlock, 2011a).

C328. Posterior (usually just the posterior-most) cervical neural arches, postzygapophyses: terminate at or beyond the posterior edge of the centrum (0); terminate in front of the posterior edge of the centrum (1) (Tschopp \& Mateus, 2013; Poropat et al., 2016).

C329. Posterior cervical neural spines, horizontal, rugose ridge immediately below spine summit on lateral surface: absent, spinodiapophyseal fossa fades out gradually dorsally (0); present, serves as distinct dorsal edge of the spinodiapophyseal fossa (1) (Tschopp \& Mateus, 2013).

C330. Posteriormost cervical and anteriormost dorsal neural spines, shape in anterior/posterior view: taper dorsally, or mediolateral width remains constant along length (0); expand dorsally, with a strongly convex dorsal margin ('paddle-shaped') (1) (González Riga, 2005; D'Emic, 2012; Poropat et al., 2016).

C331. Cervical ribs, dorsal surface of proximal portion of shaft: excavated, forming a longitudinal groove (0); unexcavated (1) (Poropat et al., 2015a, 2016).

C332. Anterior dorsal centra, ventral keel on midline: absent (0); present (1) (Mannion et al., 2012; Poropat et al., 2016).

C333. Middle-posterior dorsal centra, ventral surface: flat or transversely convex (0); transversely concave, between ventrolateral ridges (1) (Upchurch et al., 2004; Curry Rogers, 2005; Poropat et al., 2016).

C334. Middle-posterior dorsal centra, lateral pneumatic foramina divided by internal ridge/s: absent (0); present (1) (Salgado et al., 1997; Mannion et al., 2012).

C335. Anterior dorsal neural arches, shape of anterior neural canal opening: height greater than or equal to width (0); height is less than width (1) (Curry Rogers, 2005; Poropat et al., 2016).

C336. Anterior-middle dorsal neural arches, vertical midline ridge ('median infrapostzygapophyseal lamina') extending from roof of neural canal to ventral midpoint of postzygapophyses/intrapostzygapophyseal lamina (TPOL): absent (0); present (1) (González Riga, 2003; Curry Rogers, 2005, 2009; Gallina, 2011; Gallina \& Apesteguía, 2011).

C337. Anterior-middle dorsal neural arches, zygapophyseal articulation angle: between horizontal and less than $40^{\circ}$ to the horizontal (0); strongly dorsomedially oriented $\left(40^{\circ}\right.$ or more) (1) (Carballido et al., 2012; Poropat et al., 2016).

C338. Middle-posterior dorsal neural arches, neural canal in anterior view: entirely surrounded by the neural arch (0); enclosed in a deep fossa in the dorsal surface of the 
centrum (i.e. much of the canal is enclosed laterally by pedicels that are part of the centrum rather than the neural arch) (1) (Carballido et al., 2012; Poropat et al., 2016).

C339. Middle-posterior dorsal neural arches, position of parapophysis: posterior to the vertical plane defined by the anterior margin of the centrum (excluding any convex articular condyle) (0); level with, or anterior to, the vertical plane defined by the margin of the centrum (excluding any convex articular condyle) (1) (Tschopp \& Mateus, 2013).

C340. Middle-posterior dorsal neural arches, anterior centradiapophyseal lamina (ACDL): absent (0); present (1) (Mannion et al., 2012; Poropat et al., 2016).

C341. Posterior dorsal neural arches, zygapophyseal articulation angle relative to horizontal line: less than $30^{\circ}$, usually close to horizontal (0); steeply oriented, $30^{\circ}$ or greater (1) (Carballido et al., 2012; Poropat et al., 2016).

C342. Middle-posterior dorsal neural spines (single, not bifid), SPRLs: remain separate or converge at about spine midheight (or above) to form a dorsally restricted median composite lamina (SPRF well-developed and occupies the ventral half of the anterior spine surface) (0); SPRLs, if present, are short and merge into the PRSL close to the base of the spine (the PRSL may extend between the bases of the SPRLs to the top of the TPRL) (1) (Upchurch, 1995; Whitlock, 2011a; Carballido et al., 2012; Poropat et al., 2016).

C343. Middle-posterior dorsal neural spines, postspinal lamina: does not extend ventral to the neural spine (0); extends ventral to the neural spine, beyond the postzygapophyseal articular surfaces (1) (Poropat et al., 2016; note that only taxa scored as "0" for C169 are scored for this character).

C344. Middle-posterior dorsal neural spines, anterior spinodiapophyseal lamina (aSPDL): absent (0); present (1) (Upchurch et al., 2004; Poropat et al., 2016; note that the presence of an aSPDL can only be confirmed when a PSPDL is also present).

C345. Middle-posterior dorsal neural spines, SPDL bifurcates at its dorsal end to create a SPDL-F: absent (0); present (1) (Poropat et al., 2016; note that this refers to a bifurcation of the posterior SPDL when there are two SPDLs, rather than just the presence of an aSPDL and PSPDL).

C346. Sacrum, ratio of mediolateral width across sacral vertebrae and ribs (taken at midlength on the coossified sacrum) to average length of a sacral centrum: less than 4.0 (0); 4.0 or higher (1) (Upchurch, 1998).

C347. Sacral centra, ratio of mediolateral width of middle sacral centra to first and last sacral centra: approximately constant, ratio less than $1.3(0) ; 1.3$ or greater (1) (Salgado et al., 2005; D'Emic \& Wilson, 2011; Poropat et al., 2016).

C348. Sacral neural spines, all fused, forming a dorsal 'platform': absent (0); present (1) (Martínez et al., 2004; Poropat et al., 2016; revised here so that only taxa with the derived state for C174 are scored).

C349. First caudal centrum, anterior articular face shape: flat or concave (0); convex (1) (Wilson, 2002; Whitlock, 2011a; Poropat et al., 2016).

C350. Anterior-middle caudal centra (excluding Cd1), comparison of anterior and posterior articular faces of amphicoelous centra: anterior face more concave than posterior one, or these two faces are equally concave $(0)$; posterior face more deeply concave than anterior face (1) (González Riga et al., 2009; Carballido et al., 2012; D'Emic et al., 2013; Poropat et al., 2016; revised here to only score for taxa with at least some amphicoelous caudal centra in the anterior-middle caudal sequence).

C351. Middle caudal centra with convex posterior articular surface, condyle dorsally displaced: absent (0); present (1) (González Riga, 2003; Poropat et al., 2016). 
C352. Anteriormost caudal neural arches, prezygapophyses curve downwards ('droop') at their distal ends: absent (0); present (1) (Santucci \& Arruda-Campos, 2011).

C353. Anterior caudal neural spines, anterior expansion of lower portion of spinoprezygapophyseal lamina (SPRL): absent (0); present ('SPRL-process') (1) (D'Emic, 2012; Mannion et al., 2012; note that this is best observed in lateral view).

C354. First caudal rib, subtriangular process projects posteriorly at approximately midlength: absent (0); present (1) (Mannion \& Calvo, 2011; Poropat et al., 2016).

C355. Anteriormost caudal ribs, tubercle on dorsal surface at approximately midlength: absent (0); present (1) (Kellner et al., 2005; D'Emic et al., 2013; Poropat et al., 2016).

C356. Anterior-middle chevrons, articular facet surface: flat or anteroposteriorly convex (0); divided into anterior and posterior facets by a furrow (1) (Powell, 1987, 2003; Santucci \& Arruda-Campos, 2011; D'Emic, 2012).

C357. Anterior-middle chevrons, posteroventrally directed ridge or bulge on lateral surface of distal half of proximal ramus: absent (0); present (1) (Santucci \& Arruda-Campos, 2011; Poropat et al., 2016).

C358. Scapula, ventrolateral margin of acromion, anteroposteriorly concave region posterior to glenoid, followed by a flattened area: absent (0); present (1) (Poropat et al., 2015a, 2016).

C359. Scapular blade, ridge on medial surface, close to junction with acromial plate and near dorsal margin: absent (0); present (1) (Sanz et al., 1999; Upchurch et al., 2004).

C360. Scapular blade, orientation of blade long-axis with respect to coracoid articulation: more than $70^{\circ}$ (usually approximately perpendicular) (0); $70^{\circ}$ or less (1) (Wilson, 2002; Poropat et al., 2016).

C361. Coracoid, glenoid: does not expand strongly laterally relative to the lateral surface of the coracoid (0); expands prominently laterally and curves dorsolaterally so that part of the glenoid articular surface can be seen in lateral view (1) (Poropat et al., 2016).

C362. Sternal plate, shape of posterior margin in dorsal/ventral view: convex (0); straight (1) (González Riga, 2003; González Riga et al., 2009).

C363. Sternal plate, anteroposteriorly directed ridge on ventral surface, at the anterior end: absent (0); present (1) (Sanz et al., 1999; Upchurch et al., 2004).

C364. Humerus, proximal margin in anterior/posterior view: straight or convex (0); sinuous, as a result of a prominently developed process (attachment site for $M$. supracoracoideus) on the lateral margin of the proximal end (1) (Upchurch, 1998; González Riga, 2003; González Riga et al., 2009; Poropat et al., 2016).

C365. Humerus, proximal end: expands laterally relative to the shaft, giving the humerus an hourglass outline in anterior view (0); asymmetrical, with no expansion of lateral margin relative to shaft (1) (Tschopp et al., 2015a; Poropat et al., 2016).

C366. Humerus, humeral head forms a prominent subcircular process on the posterior surface of the proximal end: absent (0); present (1) (Bonaparte et al., 2006; Upchurch et al., 2015; Poropat et al., 2016).

C367. Humerus, prominent vertical ridge extends along the lateral margin of the posterior surface, from the proximolateral corner to approximately the level of the deltopectoral crest (this ridge defines the lateral margin of the lateral triceps fossa and causes this fossa to be much deeper than the medial one): absent (0); present (1) (Poropat et al., 2016).

C368. Humerus, tuberosity for attachment of the $M$. coracobrachialis on the anterior surface of the proximal third: absent (0); present (1) (Powell, 2003; Harris, 2007; Otero, 2010; Poropat et al., 2016). 
C369. Humerus, deltopectoral crest, mediolateral thickness of anterior attachment surface: approximately constant along length (0); distal half mediolaterally expanded relative to proximal half (often doubling in thickness) (1) (Wilson, 2002; Poropat et al., 2016; note that the anterior attachment surface of taxa with a medially deflected deltopectoral crest faces primarily medially).

C370. Humerus, ratio of maximum mediolateral width of distal end to proximodistal length: 0.30 or greater (0); less than 0.30 (1) (Poropat et al., 2016).

C371. Radius, beveling of distal end relative to long-axis of shaft: restricted to lateral half $(0)$; extends across the entire distal end (1) (Poropat et al., 2016).

C372. Ulna, angle between long-axes of anteromedial and anterolateral processes in proximal end view: $80^{\circ}$ or greater (usually approximately a right-angle) (0); less than $80^{\circ}$ (acute) (1) (Tschopp et al., 2015a).

C373. Metacarpals, longest metacarpal to radius proximodistal length ratio: less than 0.50 (0); 0.50 or greater (1) (Poropat et al., 2016; revised here so that only taxa scored as a " 1 " for C52 are scored).

C374. Metacarpals, metacarpal II, ratio of minimum transverse width of shaft to metacarpal length: 0.2 or higher (0); less than 0.2 (1) (Sekiya, 2011).

C375. Metacarpals, metacarpal III: longest metacarpal (0); shorter than at least one other metacarpal (1) (Curry Rogers, 2005; Poropat et al., 2016).

C376. Metacarpals, metacarpal IV, distal end profile: subrectangular (0); possesses small pointed lateral and medial projections such that the dorsal margin is longer than the ventral margin, producing a dorsoventrally compressed hexagonal or trapezoidal outline (1) (Poropat et al., 2016).

C377. Metacarpals, metacarpal V, ratio of proximal end long axis diameter to that of metacarpal I: less than 1.0 (0); 1.0 or greater (1) (D'Emic, 2012; Poropat et al., 2016).

C378. Metacarpals, metacarpal $V$, ratio of proximal end long axis diameter to that of metacarpal IV: equal or smaller (0); larger than that for metacarpal IV (1) (Poropat et al., 2015b, 2016).

C379. Metacarpals, metacarpal $\mathrm{V}$ with a medially biased flange-like swelling along proximal half of ventral surface: absent (0); present (1) (Apesteguía, 2005a; Mannion \& Calvo, 2011; Poropat et al., 2016).

C380. Ilium, ratio of dorsoventral height of iliac blade above pubic peduncle to anteroposterior length of ilium: less than 0.35 (0); 0.35 or greater (1) (Tschopp et al., 2015a; Poropat et al., 2016).

C381. Ilium, projected line (chord) connecting articular surfaces of ischiadic and pubic processes: passes ventral to ventral margin of postacetabular portion of ilium (0); passes through or dorsal to ventral edge of postacetabular portion of ilium (1) (Upchurch, 1998).

C382. Ilium, orientation of the pubic peduncle with respect to the long axis of the ilium: anteriorly deflected (0); perpendicular (1) (Salgado et al., 1997).

C383. Ilium, protuberance on the lateral surface of the ischiadic articulation: absent (0); present (1) (Borsuk-Białynicka, 1977; Poropat et al., 2015a, 2016).

C384. Pubis, proximodistally oriented ridge on lateral surface of blade, separated from the anterior margin of the pubis by a longitudinal groove: absent (0) present (1) (Powell, 2003; Salgado and Carvalho, 2008; Otero, 2010; Poropat et al., 2016).

C385. Pubis, distal end transversely expanded along lateral surface relative to shaft: present (0); absent (laminar distal blade) (1) (Curry Rogers, 2005; Poropat et al., 2016).

C386. Ischium, iliac articular surface, anteroposterior length to mediolateral width ratio: 1.0 or greater (0); less than 1.0 (1) (Poropat et al., 2016). 
C387. Ischium, ridge (for attachment of M. flexor tibialis internus III) on lateral surface of the lower part of the proximal plate/proximal portion of shaft, close to the posterior/dorsal margin of ischium: associated with parallel groove, posterior/dorsal to ridge (0); groove absent (1) (Sereno et al., 2007; D'Emic, 2012; Poropat et al., 2016).

C388. Femur, femoral head, projection: directed medially (0); directed dorsomedially (1) (Upchurch et al., 2004; Curry Rogers, 2005; Poropat et al., 2016).

C389. Femur, ratio of mediolateral breadth of tibial condyle to breadth of fibular condyle: greater than 0.8 (0); 0.8 or less (1) (Wilson, 2002; Poropat et al., 2016).

C390. Femur, shape of distal condyles: articular surface restricted to distal portion of femur (0); expanded onto anterior portion of femoral shaft (1) (Wilson \& Carrano, 1999; Wilson, 2002).

C391. Tibia to femur length ratio: less than 0.6 (0); 0.6 or greater (1) (Gauthier, 1986; Poropat et al., 2016).

C392. Fibula, articular surface of lateral trochanter: not visible in anterior view (0); visible in anterior view (1) (Poropat et al., 2016).

C393. Fibula, distal end mediolateral width to anteroposterior width ratio: 0.8 or less (0); greater than 0.8 (1) (Poropat et al., 2016).

C394. Fibula, distal end profile: elliptical or semicircular (with a straight medial margin) (0); subtriangular (with a rounded or sharper apex projecting laterally or anterolaterally where flattened anterolateral and posterolateral margins meet) (1) (Poropat et al., 2016).

C395. Metatarsals, metatarsal V, proximal end: dorsoventrally expanded relative to shaft, with a domed dorsal margin (0); not expanded relative to shaft (1) (Poropat et al., 2016).

C396. Metatarsals, metatarsal V, tubercle or ridge on ventral surface, at approximately midlength, equidistant from the medial and lateral margins: absent (0); present (1) (Poropat et al., 2016).

C397. Pedal digit I, proximal articular surface of ungual (phalanx I-2): perpendicular to long axis of ungual (0); bevelled so that the proximal articular surface faces proximolaterally and thus lies at a distinct angle to the long axis of the ungual (1) (Wilson \& Upchurch, 2009; Poropat et al., 2016).

C398. Basioccipital, fossa on lateral surface, extending from base of occipital condyle to base of basal tubera: absent (0); present (1) (Tschopp et al., 2015a).

C399. Basicranium, cranial nerve opening II (optic foramen): single opening (0); medially divided to form two foramina (1) (Sander et al., 2006).

C400. Surangular, anterior foramen: absent (0); present (1) (Tschopp et al., 2015a).

C401. Splenial, position of anterior end relative to mandibular symphysis: posterior to symphysis (0); participates in symphysis (1) (Upchurch, 1998).

C402. Teeth, D-shaped crown morphology in labial/lingual view: narrows mesiodistally along its apical third (0); narrows mesiodistally along its apical half, giving it a 'heart'-shaped outline (1) (Royo-Torres et al., 2006; Mateus et al., 2014; Mannion et al., 2017).

C403. Middle-posterior cervical neural arches, vertical midline lamina (part of the interprezygapophyseal lamina [TPRL]) divides the centroprezygapophyseal fossa (CPRF) into two fossae: absent (0); present (1) (Upchurch \& Martin, 2002; Curry Rogers, 2009).

C404. Middle-posterior cervical neural arches, vertical midline lamina (part of the interpostzygapophyseal lamina [TPOL]) divides the centropostzygapophyseal fossa (CPOF) into two fossae: absent (0); present (1) (Upchurch \& Martin, 2002; Curry Rogers, 2009).

C405. Middle cervical neural spines, lateral fossa at the base of the prezygapophyseal process bounded by SPRL, PRDL and PODL: absent (0); present (1) (Harris, 2006b; Tschopp \& Mateus, 2013). 
C406. Middle and posterior cervical neural spines, lateral surface between PRDL, PODL, SPOL (i.e. the spinodiapophyseal fossa [SDF]): has 3 or more coels separated from each other by low ridges: absent (0) present (1) (Mannion et al., 2017).

C407. Cervical ribs, anterior projection extends beyond anterior margin of centrum (including condyle): present (0); absent (1) (Mannion et al., 2017).

C408. Sacral ribs, Sv2 ribs: emanate solely from Sv2 (0); emanate from Sv2, with a contribution from Sv1 (1) (Mannion et al., 2017).

C409. Anteriormost caudal centra, ACDL: absent, or represented by no more than a faint ridge (0); present, well defined or sheet-like (1) (Wilson, 2002; Mannion et al., 2017).

C410. Anterior-middle caudal neural arches: spinopostzygapophyseal lamina (SPOL) shape: SPOL grades smoothly toward postzygapophyses (0); SPOL abruptly ends near the anterior margin of the postzygapophyseal facet, and postzygapophyses sharply set off from neural spine, often projecting as distinct processes (1) (D'Emic et al., 2016; note that this feature is usually present in the last few anterior and first few middle caudal vertebrae).

C411. Anterior-middle caudal neural arches, anteroposteriorly oriented ridge and fossa ('shoulder') between prezygapophyses and postzygapophyses: absent (0); present (1) (D'Emic et al., 2016; note that this feature is usually present in the last few anterior and first few middle caudal vertebrae).

C412. Radius, proximal to distal end anteroposterior length ratio: 0.5 or greater (0); less than 0.5 (1) (Mateus et al., 2014; Mannion et al., 2017).

C413. Ulna, vertical groove and ridge structure on posterolateral surface of distal shaft: absent (0); present (1) (Royo-Torres et al., 2006).

C414. Carpal bones, distal carpal mediolateral width to anteroposterior length ratio: less than 1.4 (0); 1.4 or greater (1) (Royo-Torres et al., 2014; Mannion et al., 2017; note that this is the largest carpal element in those taxa with more than one carpal).

C415. Metacarpal III, maximum mediolateral width to dorsoventral height of the proximal end ratio: less than $1.3(0) ; 1.3$ or greater (1) (Mannion et al., 2017).

C416. Tibia, tubercle ('tuberculum fibularis') on posterior (internal) face of cnemial crest: absent (0); present (1) (Harris, 2007; Tschopp et al., 2015a).

C417. Posterior cervical neural arches, spinodiapophyseal fossa, at base of lateral surface of neural spine: absent or shallow fossa (0); deep fossa (1) (González Riga, 2005; González Riga et al., 2009, 2018).

C418. Posterior cervical neural spines, dorsal half laterally expanded as a result of expansion of the lateral lamina (spinodiapophyseal lamina?): absent (0); present (1) (González Riga, 2005; González Riga et al., 2009, 2018; Gallina, 2011; González Riga \& Ortiz David, 2014).

C419. Anteriormost caudal neural spines, medial spinoprezygapophyseal laminae (mSPRLs) merge into the prespinal lamina (PRSL) close to the base of the spine: absent (0); present (1) (Calvo et al., 2008; Carballido et al., 2017; González Riga et al., 2018; note that in Patagotitan these might be the only SPRLs, whereas Futalognkosaurus appears to have more typical lateral SPRLs too).

C420. Metacarpals, metacarpal V, dorsomedial margin of distal third forms a prominent ridge or flange: absent (0); present (1) (González Riga et al., 2018).

C421. Metatarsals, ratio of metatarsal III to metatarsal I proximodistal length: 1.3 or greater (0); less than 1.3 (1) (González Riga et al., 2016, 2018).

C422. Metatarsals, ratio of metatarsal III to metatarsal IV proximodistal length: 1.0 or greater (0); less than 1.0 (1) (González Riga et al., 2016, 2018).

C423. Pedal digit III, number of phalanges: 3 or more (0); 2 or fewer (1) (González Riga et al., 2008, 2016; Nair \& Salisbury, 2012). 
C424. Premaxilla, external surface bears anteroventrally orientated vascular grooves originating from an opening in the maxillary contact: absent or faint (0); present and strongly developed (1) (Wilson, 2002; Sereno et al., 2007).

C425. Subnarial foramen and anterior maxillary foramen, relative position: well distanced from one another (distance equals several subnarial foramen diameters) (0); separated by narrow bony isthmus (distance equals approximately one subnarial foramen diameter) (1) (Wilson, 2002; modified here).

C426. Jugal, contribution to antorbital fenestra: absent (0); present, but small, bordering less than one-third its perimeter (1); present and large, bordering approximately one-third or more of its perimeter (2) (Wilson, 2002; Carballido et al., 2015; modified here) [ordered].

C427. Prefrontal, anterior portion of medial margin curves anteromedially to clasp the anterolateral corner of the frontal between itself and the posterior process of the prefrontal: absent (0); present (1) (Tschopp et al., 2015a).

C428. Frontals, midline contact (symphysis): patent suture (0); fused in adult individuals (1) (Salgado \& Calvo, 1992; Upchurch, 1995).

C429. Frontal, shape of suture with nasal in dorsal view: straight or slightly bowed anteriorly (0); V-shaped, pointing posteriorly (1) (Whitlock, 2011a).

C430. Frontal, dorsal surface: without paired grooves (0); grooves present, usually facing anterodorsally and extending on to nasal (1) (Whitlock, 2011a; modified here).

C431. Parietal, suture with exoccipital-opisthotic: linear, gently curved (0); sinuous (two broad subtriangular processes from the parietal invade the exoccipital-opisthotic) (1); suture not visible because of co-ossification (2) (Harris, 2006b; modified here).

C432. Postparietal foramen: absent (0); present (1) (Upchurch, 1995).

C433. Supratemporal fenestra: present, relatively large (0); almost closed or absent (1) (Wilson, 2002; Mannion et al., 2012).

C434. Quadratojugal, angle between anterior and dorsal processes in lateral view: less than or equal to $90^{\circ}$, so that the quadrate shaft is directed dorsally (0); greater than $90^{\circ}$ (usually approaching $130^{\circ}$ ), so that the quadrate shaft slants posterodorsally (1) (Gauthier, 1986; Upchurch, 1995).

C435. Squamosal, anterior extent: restricted to postorbital region (0); extends well past posterior margin of orbit (1); extends beyond anterior margin of orbit (2) (Whitlock, 2011a) [ordered].

C436. Squamosal, posteroventral margin: smooth, without ventral projection (0); with prominent, ventrally directed 'prong' (1) (Whitlock, 2011a).

C437. Pterygoid, shape of palatine ramus in lateral view: straight, at level of dorsal margin of quadrate ramus (0); stepped, dorsal margin of the palatine ramus is raised above the level of the dorsal margin of the quadrate ramus (1) (Wilson, 2002).

C438. Supraoccipital, sagittal nuchal crest: diminishes strongly in prominence ventrally, so that it barely reaches the dorsal margin of the foramen magnum (0); prominent throughout its length, so that it reaches the dorsal margin of the foramen magnum as a prominent posterior projection (1) (Whitlock, 2011a; modified here).

C439. Exoccipital, dorsolateral margin in posterior view, spur of bone curves dorsolaterally and then ventrolaterally to form the dorsomedial margin of the posttemporal fenestra: absent (0); present (1) (Xu et al., 2018).

C440. Exoccipital, small, deep, horizontally oriented groove immediately lateral to each of the proatlantal facets (where present): absent (0); present (1) (Xu et al., 2018). 
C441. Crista prootica (otosphenoidal ridge), 'leaf'-like dorsolaterally directed process near the base of the basipterygoid process: absent (0); present (1) (Salgado \& Calvo, 1992; Upchurch, 1995).

C442. Basisphenoid, area between the basipterygoid processes and parasphenoid rostrum: is a mildly concave subtriangular region (0); forms a deep slot-like cavity that passes posteriorly between the bases of the basipterygoid processes (1) (Upchurch, 1995, 1998).

C443. Basal tubera, relative prominence: anteroposterior width is approximately $33 \%$, or more, of dorsoventral height of the tubera (such tubera are distinct projections from the basipterygoid) (0); sheet-like, anteroposterior width is less than $33 \%$ (normally around $20 \%$ ) of the dorsoventral height of the tubera (such tubera are reduced to slight swellings on the ventral surface of the basipterygoid) (1) (Wilson, 2002; Whitlock, 2011a; modified here).

C444. Basal tubera, shape of posterior face: convex (0); flat or slightly concave (1) (Whitlock, 2011a; Tschopp et al., 2015a).

C445. Basal tubera, long-axes of their free distal tips in ventral view: subparallel to each other and extending transversely with respect to the long-axis of the basicranium (0); longaxis of each tuberal tip directed posteromedially, creating a ' $\mathrm{V}$ '-shape pointing towards the occipital condyle (1) (Tschopp et al., 2015a).

C446. Basal tubera, ventrolateral tip of each basal tuber projects anteriorly, giving its free distal surface an ' $L$ '-shaped profile in ventral view: absent (0); present (1) (new character: based on Royo-Torres et al., 2017a).

C447. Basisphenoid-quadrate contact: absent (0); present (1) (Wilson, 2002, 2005b; only taxa preserving a quadrate and with a raised ventrolateral lip on the posterior surface of the basal tubera are scored for this character).

C448. Basipterygoid processes, distal end transverse expansion: absent (distal end transverse width is subequal to, or less than, the transverse width at the midlength of the process) (0); expanded (marked and rapid transverse expansion close to the distal end) (1) (Tschopp et al., 2015a).

C449. Dentary, anteroventral margin shape in medial/lateral view: gently rounded (0); sharply projecting triangular process or 'chin' (1) (Upchurch, 1998; Tschopp et al., 2015a).

C450. Dentary, depth of anterior end: maintains dorsoventral height anteriorly, or increases only slightly (i.e. ratio of height of dentary at anterior end to height of dentary at midlength is 1.2 or lower) (0); increases in dorsoventral height anteriorly (i.e. ratio of height of dentary at anterior end to height of dentary at midlength is higher than 1.2) (1) (Upchurch, 1998; Wilson \& Sereno, 1998; Wilson, 2002; modified here).

C451. Dentary, tuberosity on labial surface near symphysis: absent (0); present (1) (Whitlock, 2011a).

C452. Dentary, shape of tooth row in dorsal view: follows curvature of rest of the dentary (0); bows outward relative to rest of the dentary (1) (Whitlock \& Harris, 2010; Tschopp et al., 2015a).

C453. Teeth, number of replacement teeth per alveolus: three or fewer (0); four or more (1) (Wilson, 2002).

C454. Tooth crowns, enamel thickness, asymmetric labiolingually: absent (0); present (1) (Whitlock, 2011a).

C455. Atlantal intercentrum, foramen on ventral surface, close to the posterior margin (between the posterior ventrolateral processes): absent (0); present (1) (Tschopp et al., 2015a). 
C456. Postaxial cervical centra, ridge dividing lateral pneumatic foramen: not confluent with lateral surface of remainder of centrum (0); confluent with lateral surface of remainder of centrum, dorsal and ventral to foramen (1) (new character: based on Mannion et al., 2012).

C457. Postaxial cervical centra, small fossa on posteroventral corner of lateral surface: absent (0); shallow, anteroposteriorly elongate fossa present, posteroventral to main lateral pneumatic opening (1) (Whitlock, 2011b; Tschopp \& Mateus, 2013).

C458. Anterior cervical centra, paired pneumatic fossae on anterior part of ventral surface: absent (0); present (1) (Whitlock, 2011a).

C459. Anterior cervical (unbifurcated) neural spines, prespinal lamina: absent (0); present (1) (Curry Rogers, 2005; polarity reversed here).

C460. Middle cervical neural spines, orientation of anterior margin in lateral view: vertical or sloping posterodorsally (0); anteriorly inclined (1) (Rauhut et al., 2005).

C461. Middle cervical neural spines, angle between PODL and SPOL in lateral view: acute, less than 85 degrees (usually close to 45 degrees) (0); 85 degrees or more (usually 90 degrees) (1) (Rauhut et al., 2005; Whitlock, 2011a).

C462. Posterior cervical neural spines, sharp-lipped dorsoventrally elongate coel on lateral surface, located in anterior part of SDF: absent (0); present (1) (Mannion et al., 2012).

C463. Posterior cervical and/or anterior dorsal bifid neural spines, morphology of metapophyses in anterior view: widely diverging (0); narrow, parallel to converging (1) (Rauhut et al., 2005).

C464. Cervical ribs, distal blade: single (0); bifurcates into dorsal and ventral processes (1) (new character: based on Royo-Torres et al., 2006; Britt et al., 2017).

C465. Anterior-middle cervical ribs, tuberculum in lateral view: nearly vertical (0); oriented posterodorsally (1) (Tschopp et al., 2015a).

C466. Dorsal centra, anteroposterior length (excluding articular condyle), increases or remains approximately the same along the sequence (0); shortens from anterior to posterior dorsal vertebrae (1) (Mannion et al., 2012; modified here).

C467. Anteriormost dorsal diapophyses, fossa on anterior surface, close to distal end: absent (0); present (1) (new character; note that this is a second fossa, lateral to the prezygapophyseal centrodiapophyseal fossa [PRCDF]).

C468. Anteriormost dorsal neural spines, epipophyses: absent (0); present (1) (new character; note that taxa that lack epipophyses in presacral vertebrae altogether [i.e. that are scored as ' 0 ' for C127] are not scored for this character).

C469. Anteriormost dorsal neural spines, height relative to spinopostzygapophyseal laminae (and epipophyses, where present): project well beyond SPOLs (0); approximately level with SPOLs (1) (new character).

C470. Dorsal neural spines (excluding anteriormost dorsal vertebrae), ventral portion of spinoprezygapophyseal lamina (SPRL): contacts the prezygapophysis (0); contacts the diapophysis following 'lamina capture' (1) (new character: based on Wilson, 2012).

C471. Dorsal neural spines, spinodiapophyseal webbing: laminae follow curvature of neural spine and diapophysis in anterior view (0); laminae "festooned" from spine, dorsal margin does not closely follow shape of neural spine and diapophysis (1) (Sereno et al., 2007).

C472. Middle and posterior dorsal neural arches, paired, sharp-lipped fossae within arch fossa, dorsolateral to neural canal and medial to CPRLs/CPOLs: absent (0); present as shallow excavations (1); present and extend anteroposteriorly throughout the neural arch as foramina (2) (Sereno et al., 2007; Whitlock 2011a; Tschopp et al., 2015a; Wilson \& Allain, 2015; modified here) [ordered]. 
C473. Middle and posterior dorsal neural arches, centroprezygapophyseal lamina (CPRL): single, undivided (0); bifurcates (or excavated) toward upper end, forming a centroprezygapophyseal lamina fossa (CPRL-F ['anterior infrazygapophyseal fossa']) (1) (Upchurch, 1995; Curry Rogers, 2005; D'Emic et al., 2013).

C474. Middle and posterior dorsal neural arches, shape of centropostzygapophyseal lamina (CPOL): single (0); divided, left and right medial CPOLs uniting beneath hyposphene, lateral CPOLs extending towards the anterior part of each postzygapophysis and/or merging into the posterior face of the PCDL (1) (Wilson, 2002; modified here).

C475. Middle and posterior dorsal neural arches, morphology of prezygapophyses: separated from each other by a gap or TPRL on the midline (0); confluent (1) (new character: based on Apesteguía et al., 2010; Wilson \& Allain, 2015).

C476. Middle and posterior dorsal neural arches, prezygoparapophyseal lamina (PRPL): absent (0); present (1) (Wilson, 2002).

C477. Posterior dorsal neural arches, parapophysis position: ventral to or level with the prezygapophysis (0); dorsal to the prezygapophysis (1) (Whitlock, 2011a).

C478. Posterior dorsal neural arches, accessory lamina in POCDF linking hyposphene to PCDL: absent (0); present (1) (Mannion et al., 2012; Tschopp et al., 2015a; taxa that lack a hyposphene are scored with state '?').

C479. Middle-posterior dorsal transverse processes, anterior surface excavated by a mediolaterally elongate, channel-like fossa or fenestra (laterodiapophyseal fossa): absent (0); present (1) (new character: based on Ibiricu et al., 2013).

C480. Middle-posterior dorsal neural spines, shape in anterior/posterior view: rectangular through most of length (0); 'petal' shaped, expanding transversely through $75 \%$ of its length and then tapering (1) (Calvo \& Salgado, 1995; Upchurch, 1998; Whitlock, 2011a; Mannion et al., 2012; taxa that lack dorsal vertebrae but that have this morphology in the sacral/anteriormost caudal vertebrae are also scored with the derived state).

C481. Middle and posterior dorsal neural spines, accessory lamina on lateral surface, extending posterodorsally from the spinoprezygapophyseal lamina to the spinopostzygapophyseal lamina: absent (0); present (1) (Tschopp et al., 2015a).

C482. Middle and posterior dorsal neural spines, divided SPOL: bifurcates a short distance above the postzygapophysis (0); divided into lateral and medial branches throughout its length (1) (Whitlock, 2011a; modified here so that only taxa with a divided SPOL are scored).

C483. Middle and posterior dorsal neural spines, lateral spinopostzygapophyseal lamina (ISPOL): extends to posterolateral margin of spine (0); does not reach lateral margin of the spine, because it fades out on the posterior surface or extends dorsomedially to contact the postspinal lamina (i.e. forms a second medial SPOL) (1) (new character: based on Wilson et al., 2011; note that if only a single undivided SPOL is present, then the state score is determined on the basis of whether this extends to the posterolateral margin of the spine or not).

C484. Middle and posterior dorsal neural spines, spinoparapophyseal lamina (SPPL): absent (0); present (1) (Wilson \& Allain, 2015).

C485. Posterior dorsal neural spines, ratio of mediolateral width to anteroposterior length (at base): less than 1.0 (longer than wide) (0); 1.0 or greater (wider than long) (1) (Upchurch, 1998; Wilson, 2002).

C486. Posterior dorsal and/or sacral neural spines, dorsoventral height to centrum length ratio: 2.0 or greater (0); less than 2.0 (2) (Upchurch, 1998; Wilson, 2002; polarity reversed here). 
C487. Thoracic ribs, area between capitulum and tuberculum, on the posterior surface at the base of the rib head: flat (0); oblique ridge connects the medial and lateral edges (1) (Tschopp et al., 2015a).

C488. Sacral neural spines, lateral surface anterior and posterior to SPDL: unbroken bone surface (0); excavated by numerous foramina (1) (new character: based on Wilson \& Allain, 2015).

C489. Anterior caudal centra, anteroposterior length: subequal along first 20 vertebrae (0); increasing in length by a factor of 1.5 or greater over first 20 vertebrae (1) (Upchurch, 1998).

C490. Anteriormost caudal centra, anterior centrodiapophyseal lamina (ACDL): single (0); divided (1) (Wilson, 2002; only taxa with an ACDL are scored for this character).

C491. Anteriormost caudal neural arches, postzygodiapophyseal lamina (PODL): absent (0); present (1) (new character: based on Wilson, 2002).

C492. Anteriormost caudal neural arches, centroprezygapophyseal fossa (CPRF) between dorsal margin of anterior neural canal opening and below interprezygapophyseal lamina (TRPL): absent (0); present (1) (new character: based on Remes, 2006).

C493. Anterior caudal neural arches, prezygapophyseal midline 'boss' ventral to prezygapophyses: absent (0); present (1) (new character: based on Mannion et al., 2011).

C494. Anterior caudal neural spines, shape: single (0); slightly bifurcate (1) (Whitlock, 2011a).

C495. Anterior caudal neural spines, dorsal margin in lateral view: flat or convex (0); concave (1) (new character).

C496. Anterior caudal neural spines, SPRL: absent, or restricted to the anterolateral margin of the spine (0); present, extending onto lateral aspect of neural spine (1) (Wilson, 2002; Mannion et al., 2012; modified here).

C497. Anterior caudal neural spines, lateral lamina: absent (0); present (1) (Wilson, 1999).

C498. Anterior caudal neural spines, lateral margin of lateral lamina: retains the same anteroposterior width along its length (0); expands anteroposteriorly towards its dorsal end (1) (Tschopp et al., 2015a).

C499. Anterior caudal neural spines, elliptical depression between lateral lamina and spinopostzyapophyseal/postspinal lamina on lateral neural spine: absent (0); present (1) (Sereno et al., 2007; Tschopp et al., 2015a).

C500. Anterior caudal neural spines, triangular lateral processes: absent (0); present (1) (Sereno et al., 2007).

C501. Anteriormost caudal ribs (excluding the first), distal tip ventrally deflected, such that the ventral margin of the caudal rib is strongly concave in anterior/posterior view: absent (0); present (1) (Gallina et al., 2014; modified here).

C502. Anteriormost caudal ribs, orientation of ventral surface in anterior/posterior view: lateral (less than 30 degrees to the horizontal plane) (0); dorsolateral (at $30^{\circ}$ or more to the horizontal plane, typically $40-50^{\circ}$ ) (1) (Whitlock, 2011a; modified here; note that this is based on the entire length of the rib, rather than just the medial portion).

C503. Anteriormost caudal ribs, anterior surface: unexcavated (0); excavated, forming a sharp-lipped fossa or foramen (1) (new character: based on Mannion et al., 2011).

C504. Anteriormost caudal ribs, excavated anterior surface internally subdivided by subvertical ridges: absent (0); present (1) (new character: based on Mannion et al., 2011).

C505. Anterior caudal ribs, position: situated on the centrum and arch (0); restricted to the neural arch and dorsal margin of centrum (1) (new character: based on Mannion et al., 2011). 
C506. Middle caudal centra, ventral longitudinal hollow: absent (0); present (1) (McIntosh, 1990; Upchurch, 1995, 1998; Wilson, 2002; revised here to separate anterior and middle caudal vertebrae).

C507. Middle caudal centra, distinct ventrolateral ridges, extending the full length of the centrum: absent (0); present (1) (McIntosh, 1990; Upchurch, 1995, 1998; Upchurch et al., 2004; Mannion et al., 2013; revised here to separate anterior and middle caudal vertebrae).

C508. Middle caudal centra, articular surface shape: cylindrical (ventral and lateral surfaces merge smoothly into each other) (0); subtriangular, with transversely wide ventral margin (1) (Wilson, 2002; Gallina \& Apesteguía, 2005; Mannion et al., 2012).

C509. Middle caudal centra, lateral pneumatic openings: absent or present as shallow fossa (0); present, with sharply defined margins (1) (Gallina et al., 2014; modified here).

C510. Scapula, anteroposterior length of acromion to total scapula length ratio: less than 0.4

(0); 0.4 to < 0.5 (1); 0.5 or greater (2) (Gallina \& Apesteguía, 2005; modified here) [ordered].

C511. Scapula, posteriorly directed hook-like acromion process, separated from the remaining dorsal margin of the acromion by a concavity: absent (0); present (1) (Gallina \& Apesteguía, 2005; Mannion, 2009).

C512. Scapula, acromial ridge: absent, or only very weakly developed (0); present and welldeveloped, so that a clear ridge is present and defines the posterior margin of a distinct fossa on the lateral surface of the acromion (1) (Upchurch, 1995; modified here).

C513. Scapula, bulge-like muscle scar on medial surface, at approximately midheight, just distal to the junction between the acromion and scapular blade: absent (0); present (1) (Whitlock, 2011a; modified here).

C514. Scapula, ratio of anteroposterior length to minimum blade dorsoventral height: 5.5 or higher (0); less than 5.5 (1) (Carballido et al., 2012; modified and polarity reversed here).

C515. Scapula, highest point of the dorsal margin of the blade: lower than the dorsal margin of the proximal end (0); at the same height as the dorsal margin of the proximal end or higher (1) (new character: based on Mannion, 2009; Carballido et al., 2012).

C516. Humerus, angle between long-axes of proximal and distal articular surfaces: $30^{\circ}$ or less (proximal and distal end long-axes lie in approximately the same plane) (0); twisted through an angle of $30^{\circ}$ or more (usually more than $40^{\circ}$ ) (1) (Gilmore, 1932; Tschopp et al., 2015a).

C517. Humerus, distal condyles: extend the same distance distally, or lateral distal condyle extends further distally than medial distal condyle (0); distal end bevelled as a result of the medial distal condyle extending further distally than the lateral condyle (1) (new character).

C518. Humerus, lateral margin in distal view: deflected such that it faces entirely posterolaterally (0); flat (facing laterally) or convex (1) (new character; the anterior margin [excluding condyles] should be orientated horizontally when scoring this character).

C519. Manual phalanx I-1, lapet-like projection from proximodorsal margin, overhanging proximal articular surface: present (0); absent (1) (new character).

C520. llium, profile of dorsal margin in lateral view: gently convex, with a nearly straight portion centrally (0); strongly convex throughout its length, following an arc of a circle (1) (Wilson, 2002; Tschopp et al., 2015a; modified here).

C521. Pubis, proximal surface and proximal third of anterior margin, in lateral view: meet at an acute angle (0); meet at a right angle (1) (new character). 
C522. Pubis, development of ambiens process: absent or small and confluent with anterior margin of pubis (0); prominent, projecting anteriorly (1) (Mclntosh, 1990; Upchurch, 1998; modified here).

C523. Pubis, ambiens process ventral (distal) surface in lateral view: flat or convex (0) concave so that the ambiens process is 'hooked' (1) (Mclntosh, 1990; Upchurch, 1995, 1998; only taxa with a well-developed ambiens process scored for this character).

C524. Pubis, obturator foramen: entirely ringed by bone (0); open posteriorly (1) (new character: based on Carballido et al., 2012; note that taxa are only scored with the derived state when the individual is an adult).

C525. Pubis, distal end, expands anteriorly and posteriorly (approximately equally): absent (0); present (1) (new character: based on Salgado et al., 2004).

C526. Ischium, iliac peduncle in lateral view: straight or widening in smooth curve distally (0); narrow, with distinct "neck" (1) (Sereno et al., 2007).

C527. Ischium, acetabular articular surface: maintains approximately the same transverse width throughout its length (0); transversely narrower in its central portion and strongly expanded as it approaches the iliac and pubic articulations (1) (Mannion et al., 2012).

C528. Ischium, angle formed between the long axis of the shaft and the acetabular line (i.e. the straight line from the anterodorsal corners of the iliac and pubic peduncles) in lateral view: $80^{\circ}$ or greater (0) acute, less than $80^{\circ}$ (typically less than $70^{\circ}$ ) (1) (Carballido et al., 2012).

C529. Ischium, dorsoventral height of pubic articulation: less than or equal to the anteroposterior length of the proximal plate $(0)$; greater than the anteroposterior length of the proximal plate (1) (Salgado et al., 1997; Carballido et al., 2012).

$\mathrm{C530}$. Ischium, ridge (for attachment of M. flexor tibialis internus III) on lateral surface of the lower part of the proximal plate/proximal portion of shaft, close to the posterior/dorsal margin of ischium: transversely rounded throughout its length (0); thin and sharp (1) (Sereno et al., 2007; modified here).

C531. Ischium, ventral margin of proximal plate in lateral view: flat along its length (0); has a ventral, subtriangular projection at its anterior end (1) (new character; only taxa whose ischia are emarginate distal to the pubic articulation are scored for this character).

C532. Ischium, shape of distal shaft in transverse cross-section: triangular, depth of ischial shaft increases medially (0); blade-like, medial and lateral depths subequal (1) (Wilson, 2002; polarity reversed here).

C533. Ischium, morphology of conjoined distal ends: ' $V$ '-shaped, forming an angle of $90^{\circ}$ or less (usually approximately $50^{\circ}$ ) with each other (0); forming an angle of more than $90^{\circ}$ (typically flat, nearly coplanar) (1) (Upchurch, 1998; Wilson \& Sereno, 1998).

C534. Femur, fourth trochanter: present as low rounded ridge (0); greatly reduced so that it is virtually absent (1) (Mannion et al., 2012).

C535. Femur, ratio of greatest anteroposterior thickness of shaft (excluding any contribution from the fourth trochanter) to greatest anteroposterior width across distal end: less than or equal to 0.5 (0); greater than 0.5 (1) (Whitlock, 2011a).

C536. Tibia, lateral margin of cnemial crest in anterior view: rounded (0); subtriangular, forming a point (1) (Tschopp et al., 2015a).

C537. Fibula, distal tip of lateral muscle scar: proximal, located above midshaft (0); located approximately at mid-shaft (1) (Whitlock, 2011a; polarity reversed here).

C538. Astragalus, fibular articular surface: faces laterally or dorsolaterally (0); faces posterolaterally because its anterior margin projects laterally (1) (Whitlock, 2011a). 
C539. Metatarsals I-III, rugosities on dorsolateral margins near distal ends: absent (0); present (1) (Upchurch, 1995).

C540. Metatarsal I, tubercle/rugosity on medial surface, situated at approximately midlength and equidistant from dorsal and ventral margins: absent (0); present (1) (new character: based on D'Emic et al., 2016).

C541. Metatarsal II, distal condyle, ventrolateral projection: absent (0); present (1) (Tschopp et al., 2015a).

C542. Pedal phalanx I-1, proximal and ventral surfaces: meet at approximately $90^{\circ}(0)$; proximoventral corner drawn out into thin plate underlying metatarsal I (1) (Mclntosh et al., 1992). 


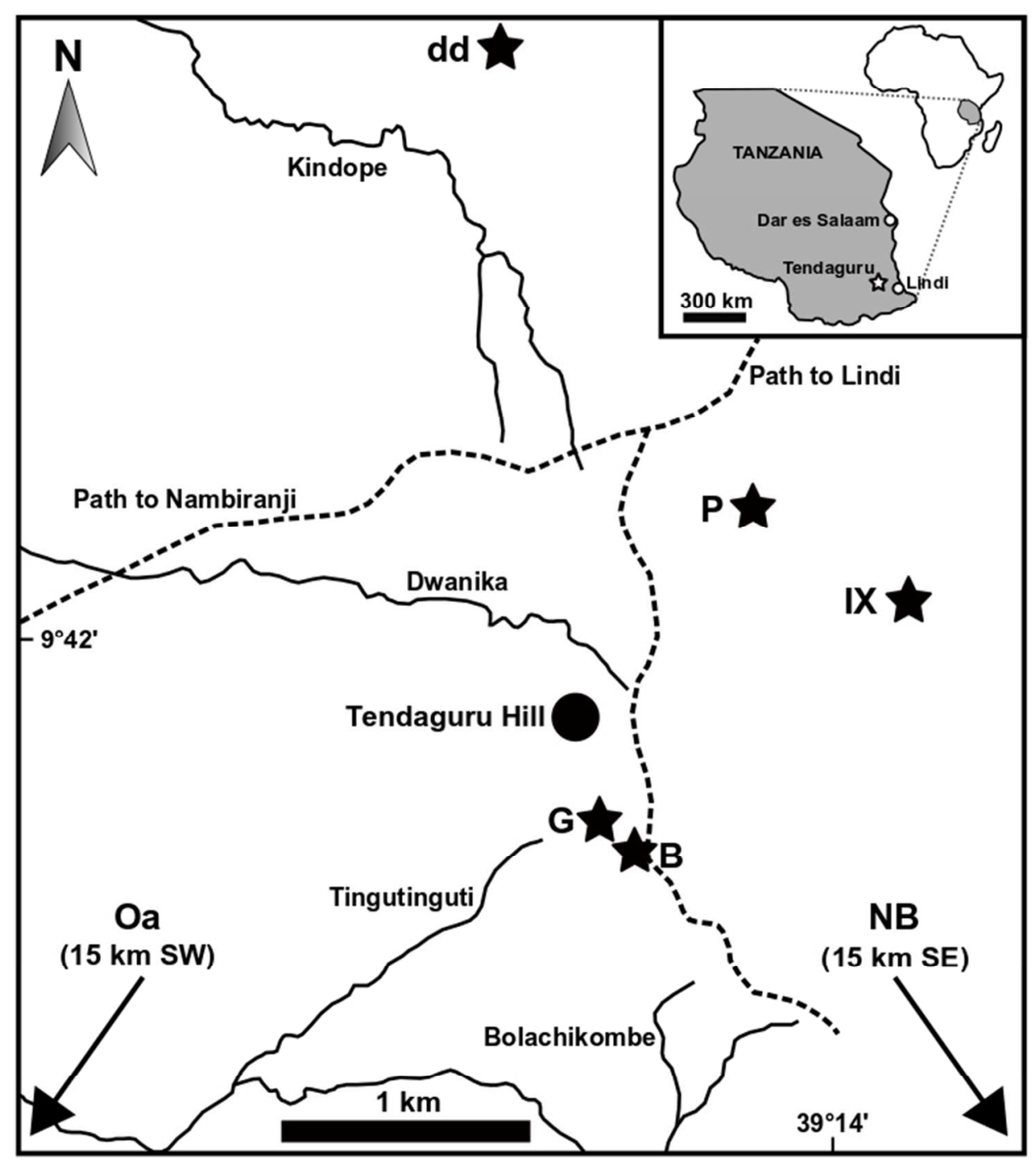


A

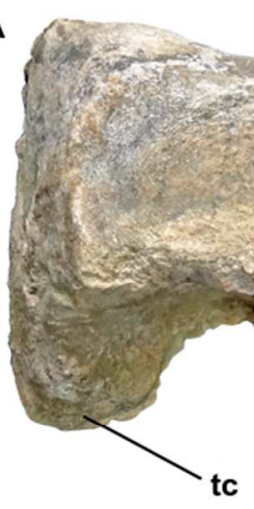

c

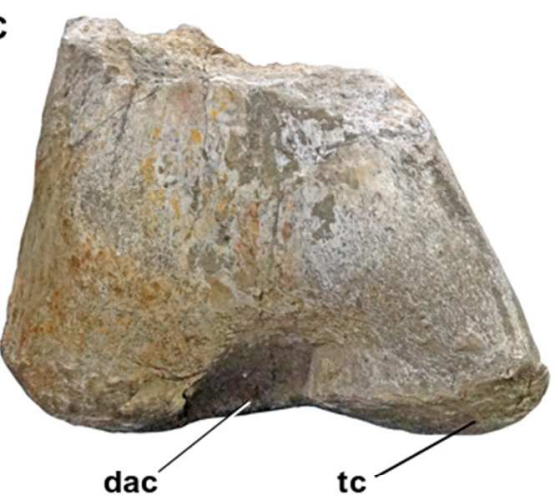

D

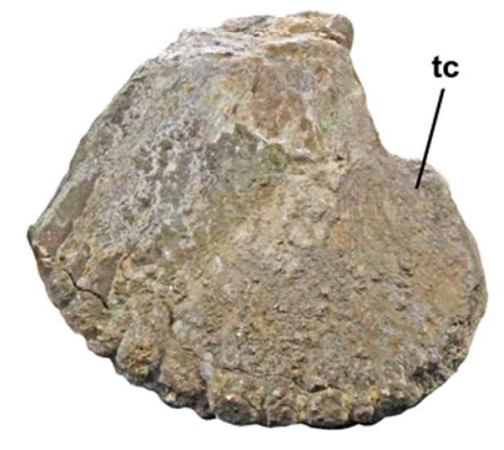

E

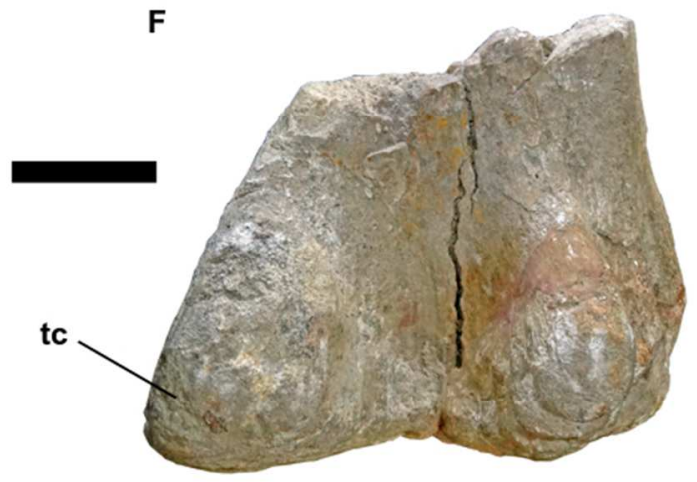




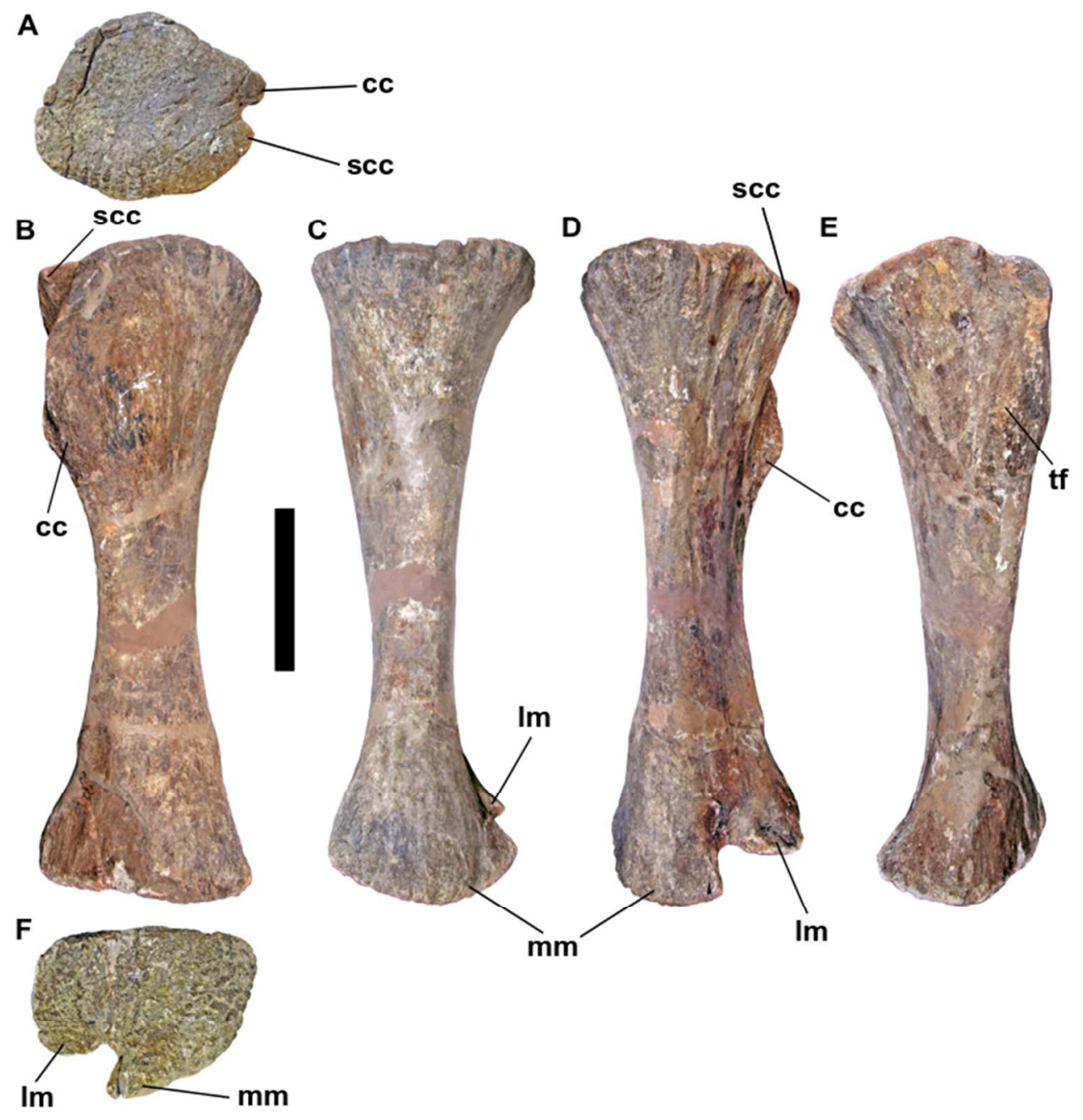


A

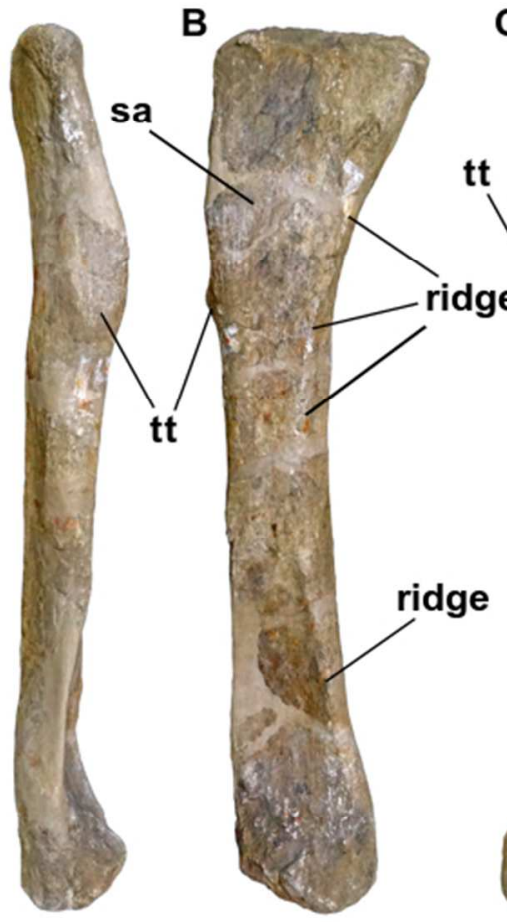

C<smiles>[3H][C@@H]1[CH]CC1</smiles>

D

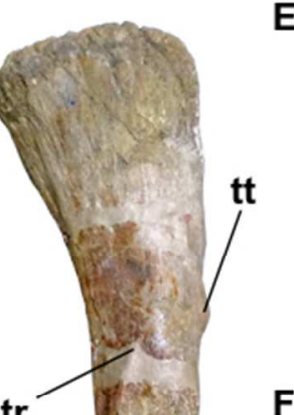

F
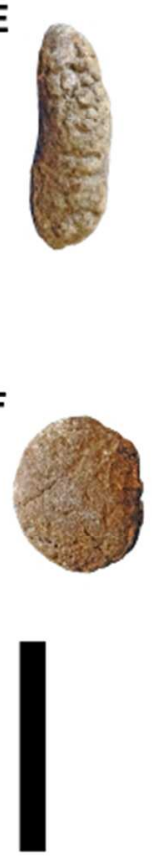


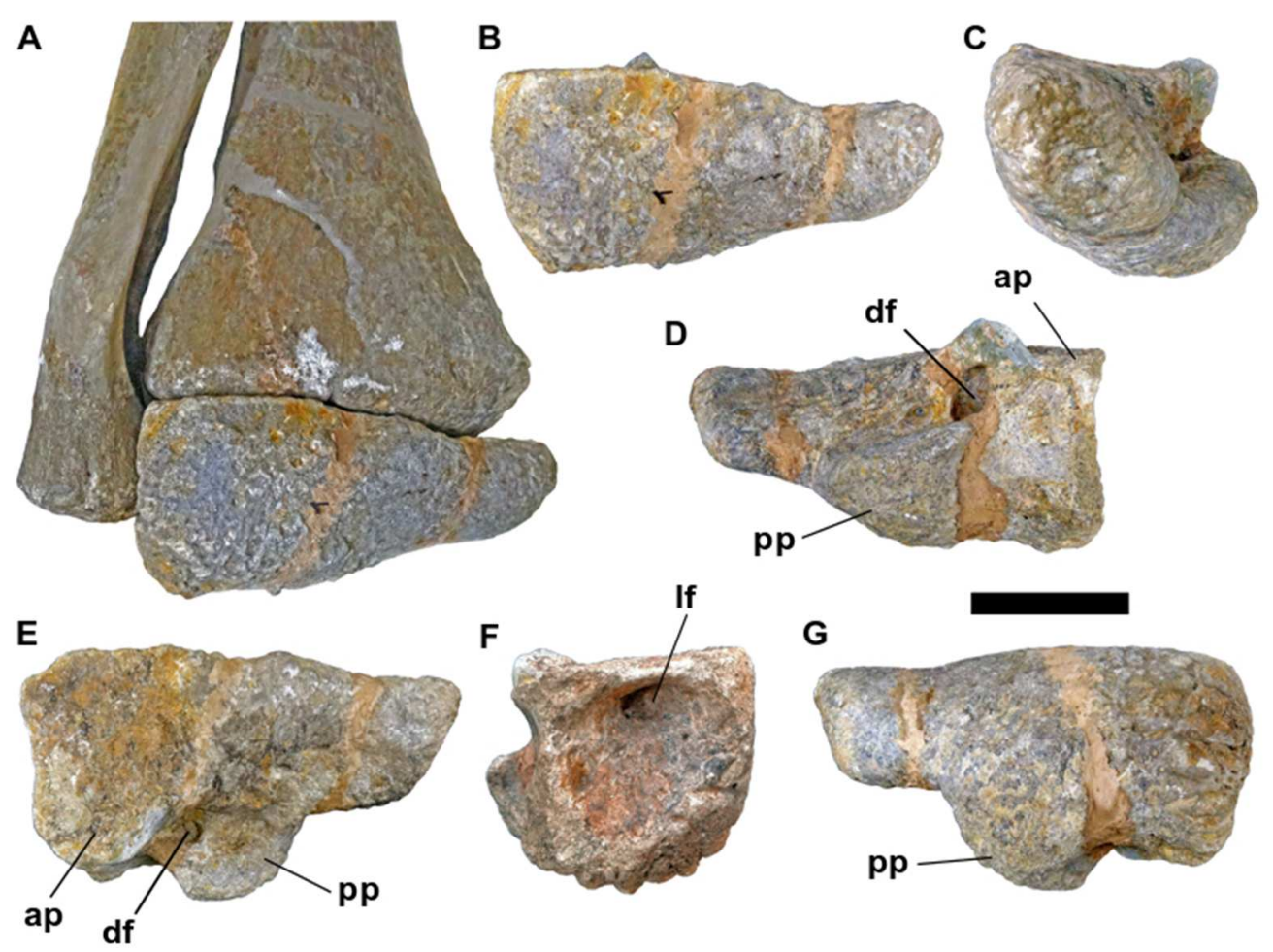




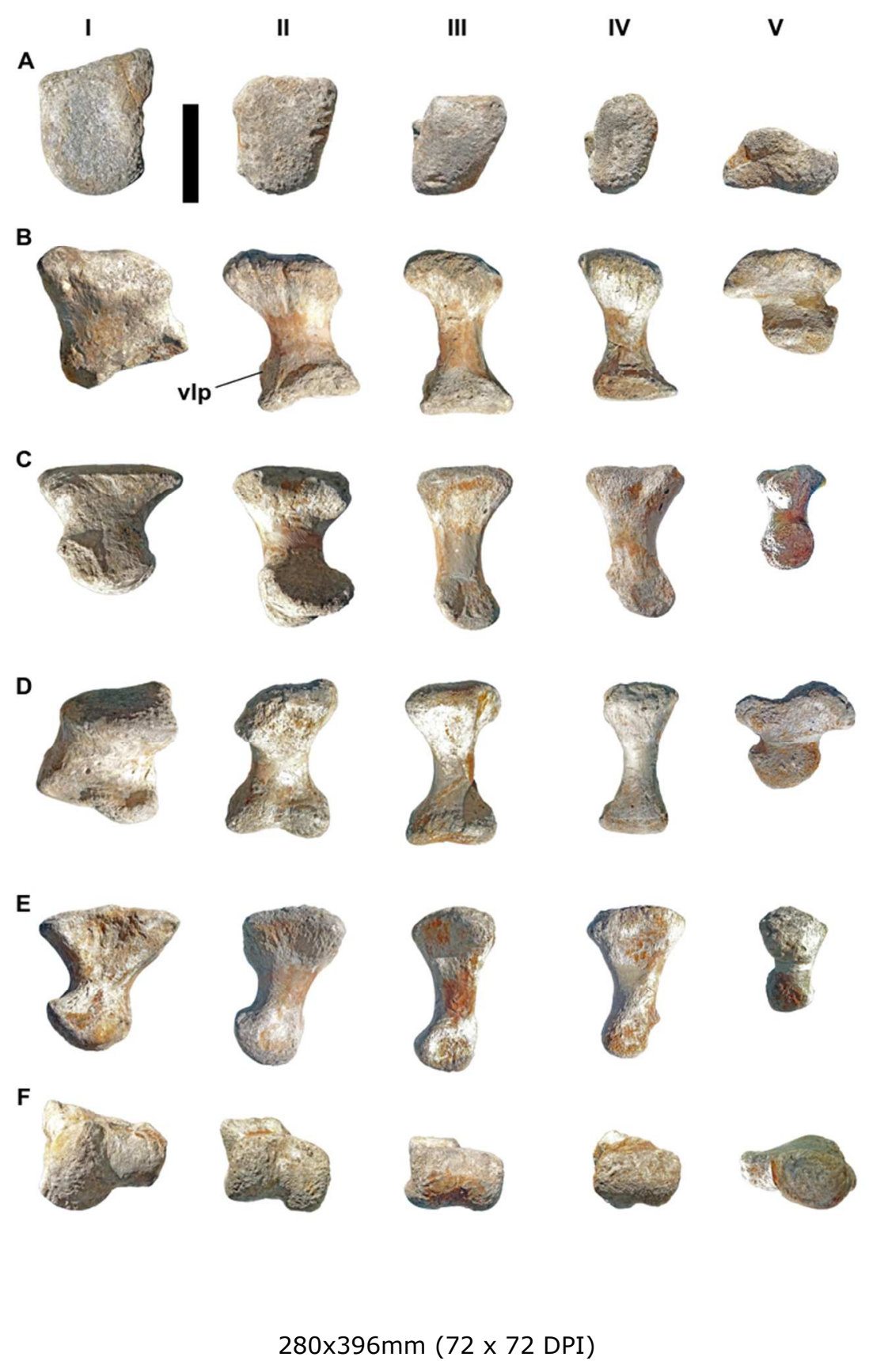



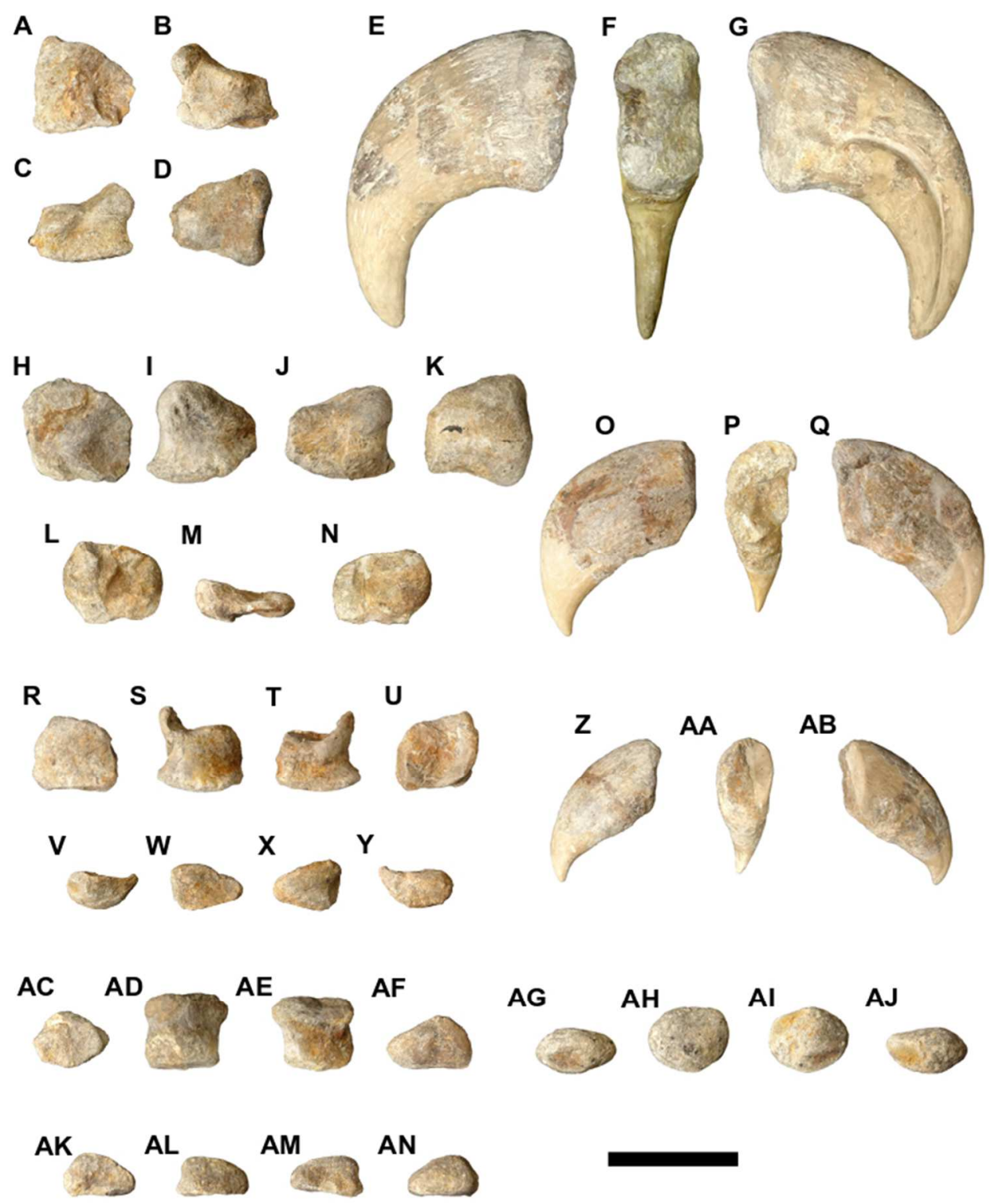


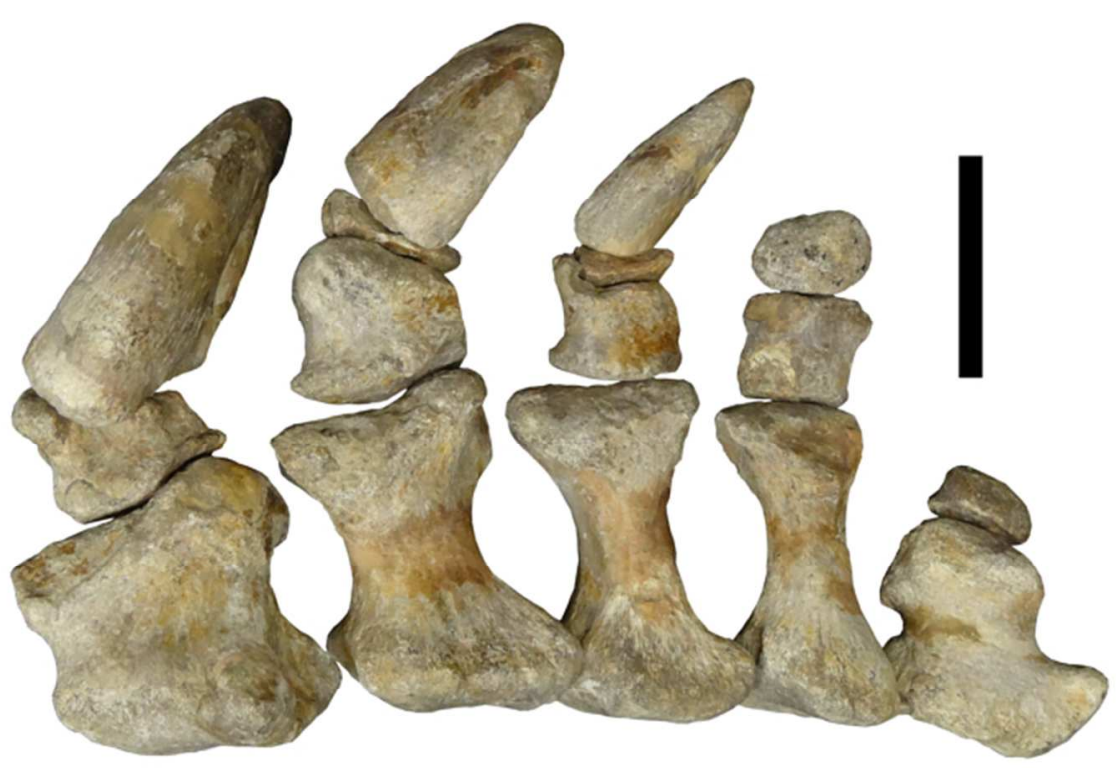




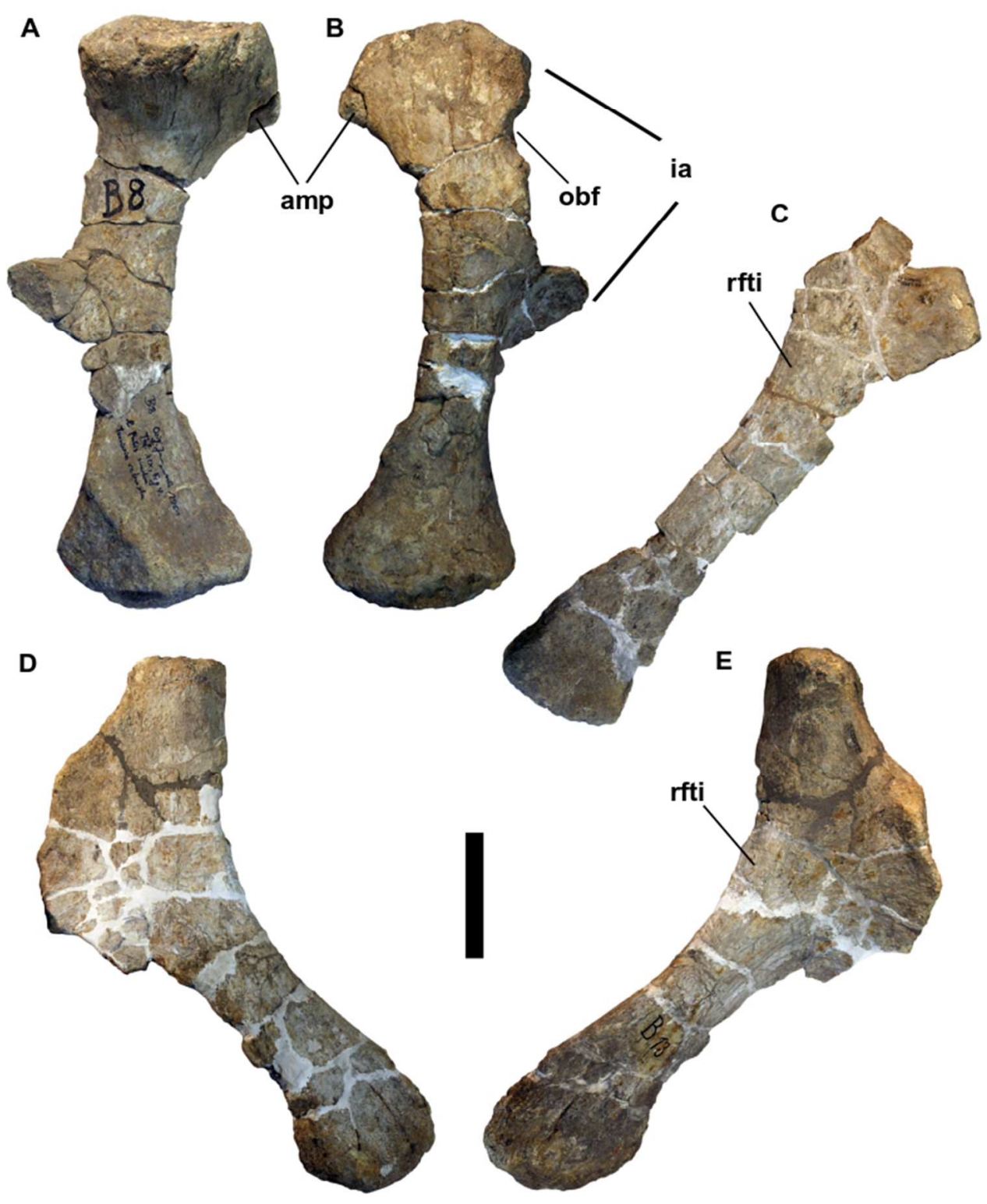




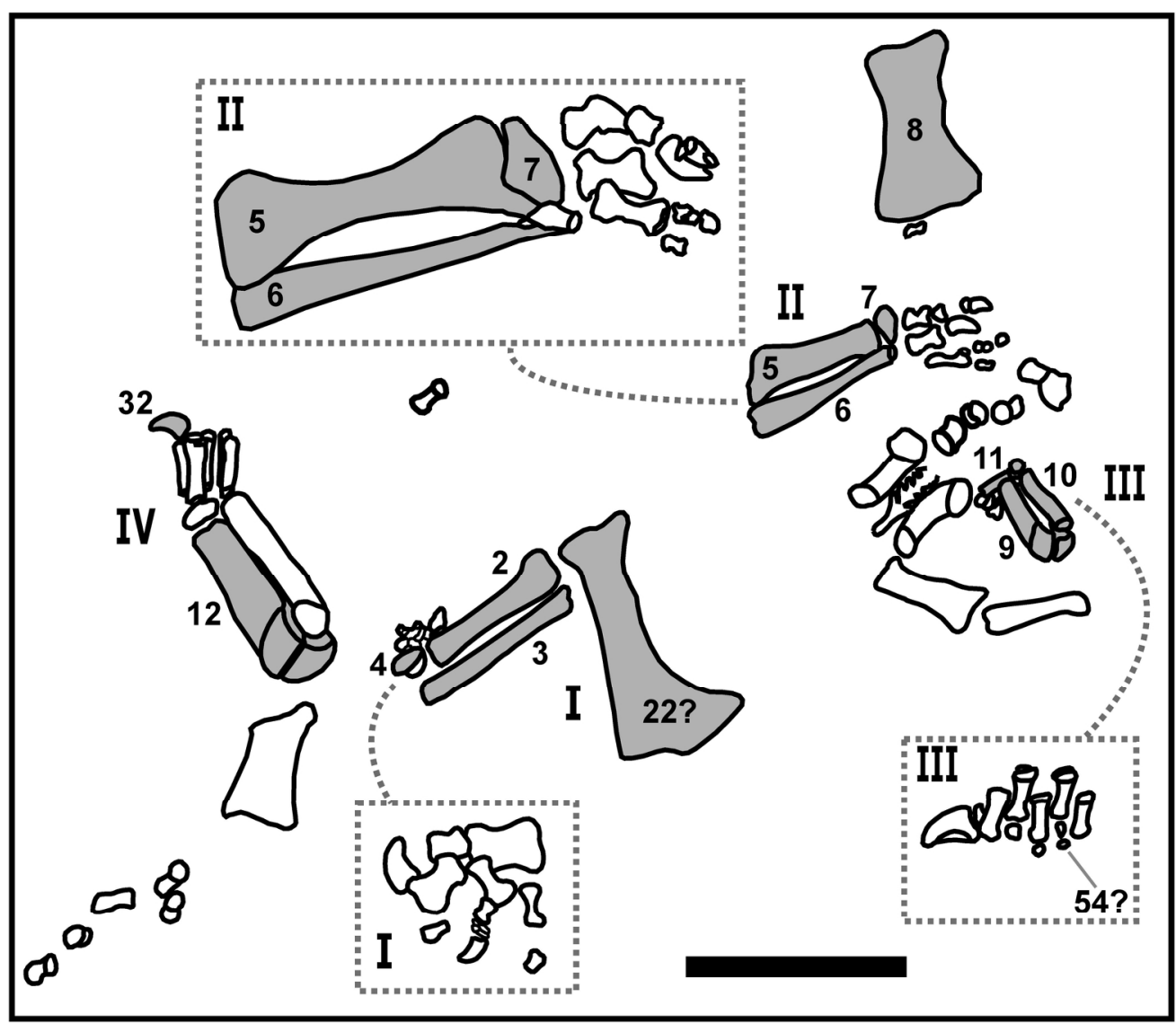

Figure 10. Tendaguru Formation Quarry P excavation sketch of Janenschia robusta referred material. Remaining material in the MfN is infilled with grey. Roman numerals refer to the four individuals designated by Bonaparte et al. (2000), and the other numbers correspond to the P Quarry field numbers assigned by Janensch (see text for more details). Redrawn from Bonaparte et al. (2000), based on the field notebook of Janensch. Scale bar $=1000 \mathrm{~mm}$ [intended for double column width]. 

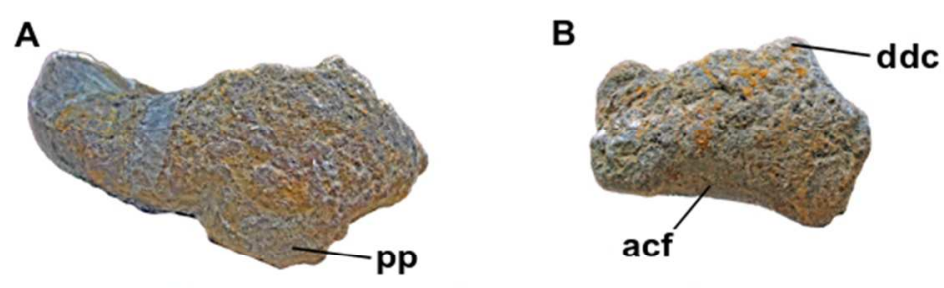

C

D E 


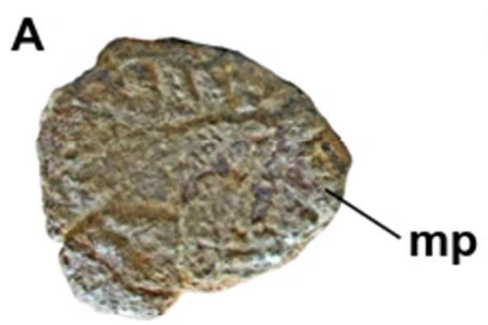

B

C
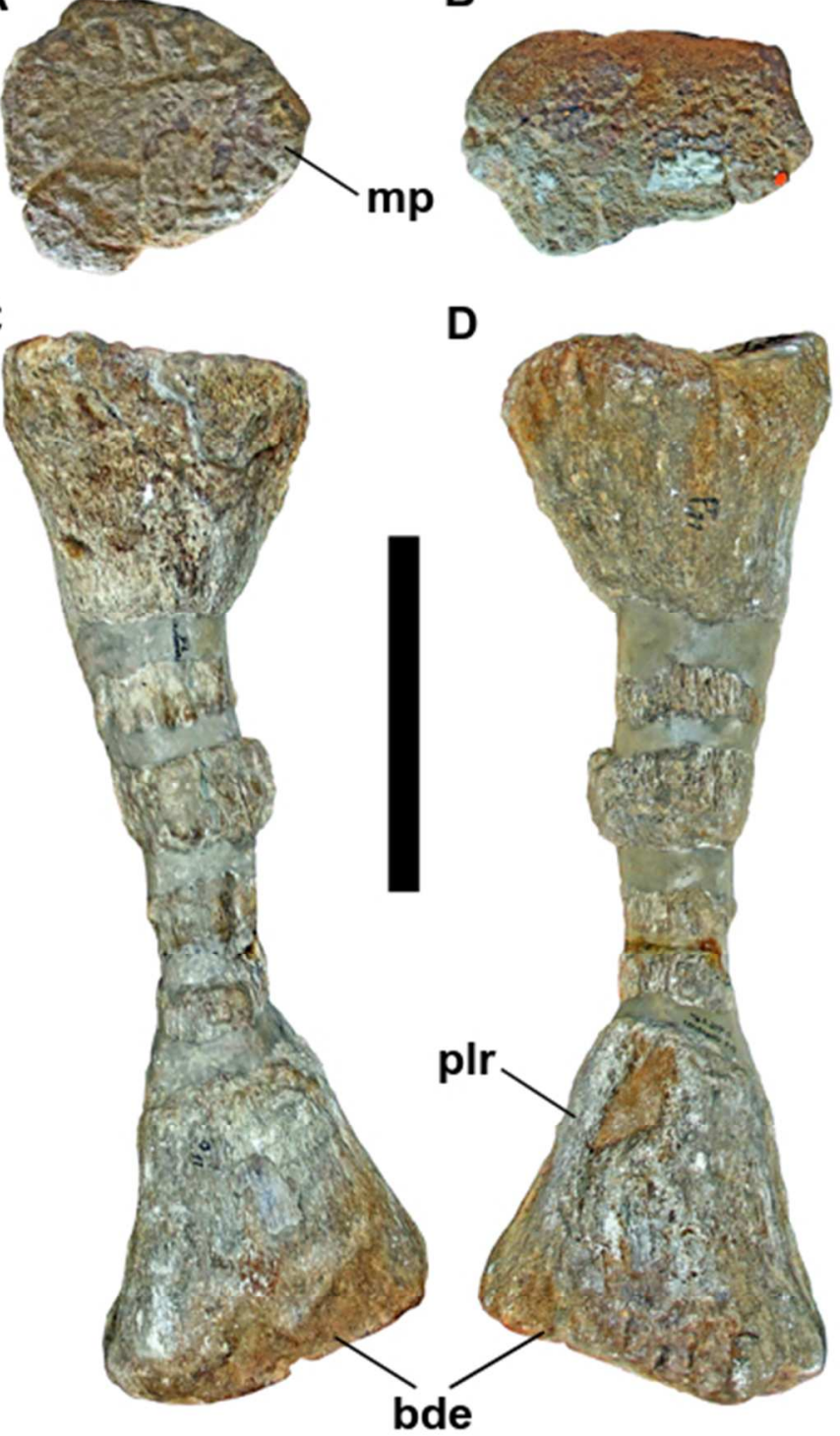


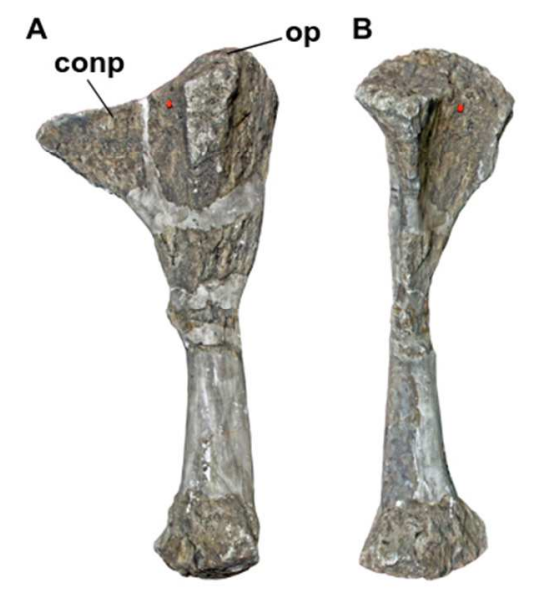

C
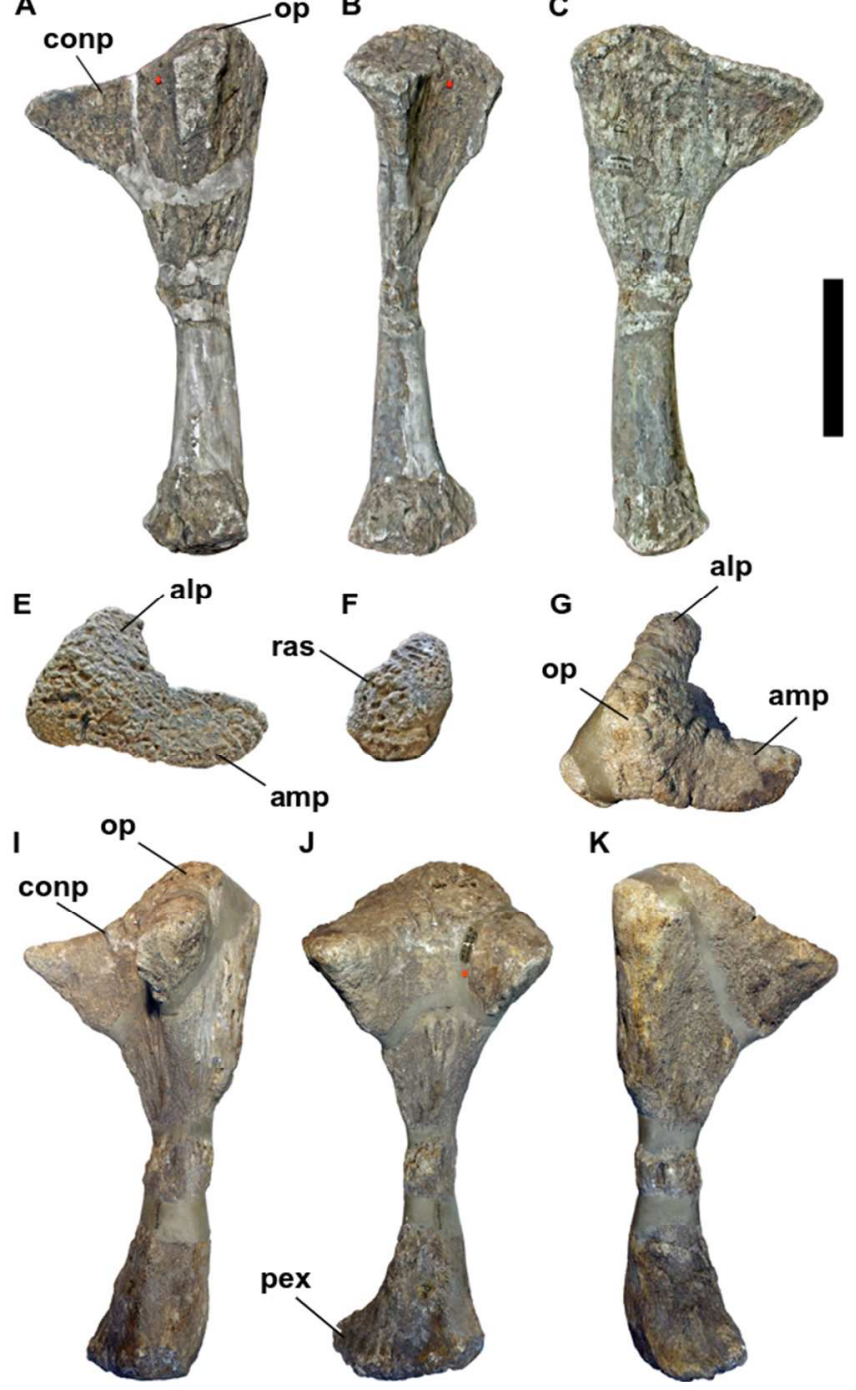

K

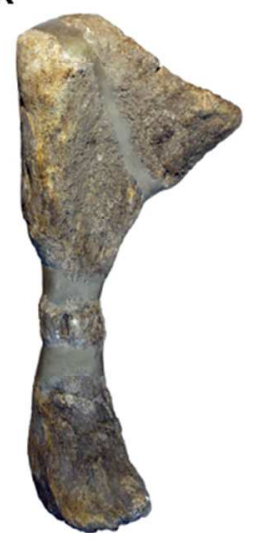

D

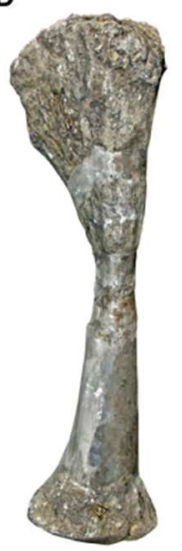

H

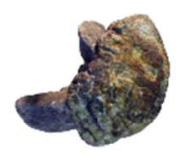

$\mathbf{L}$

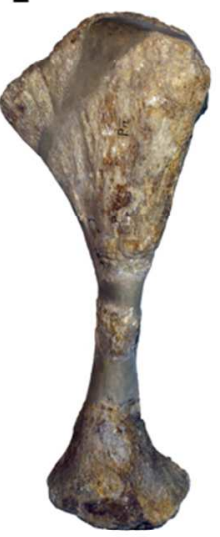
$280 \times 396 \mathrm{~mm}(72 \times 72 \mathrm{DPI})$ 
A

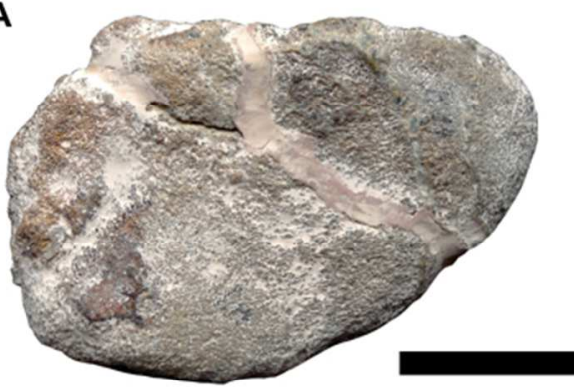

C

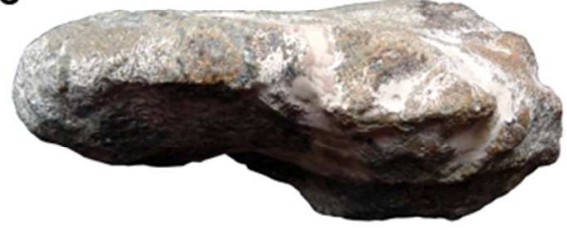

E

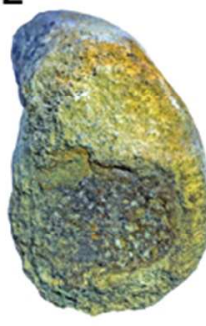

B

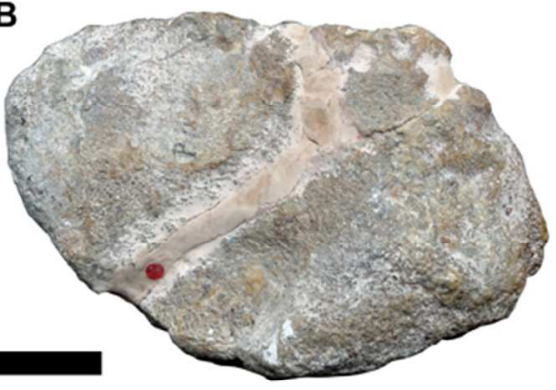

D

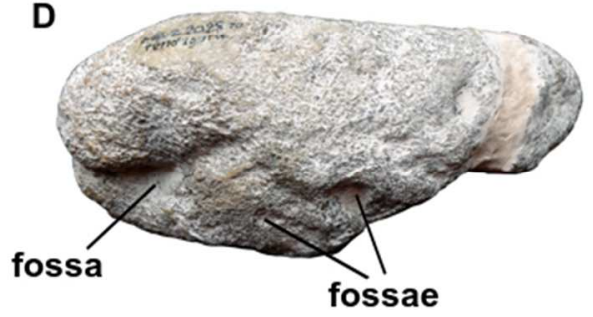

G

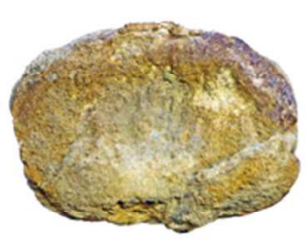

H

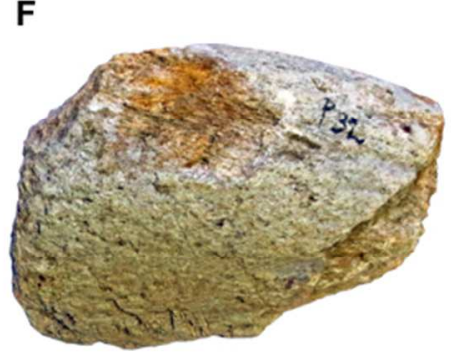

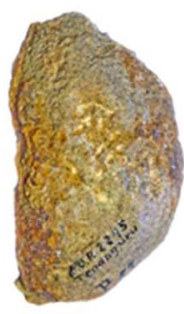




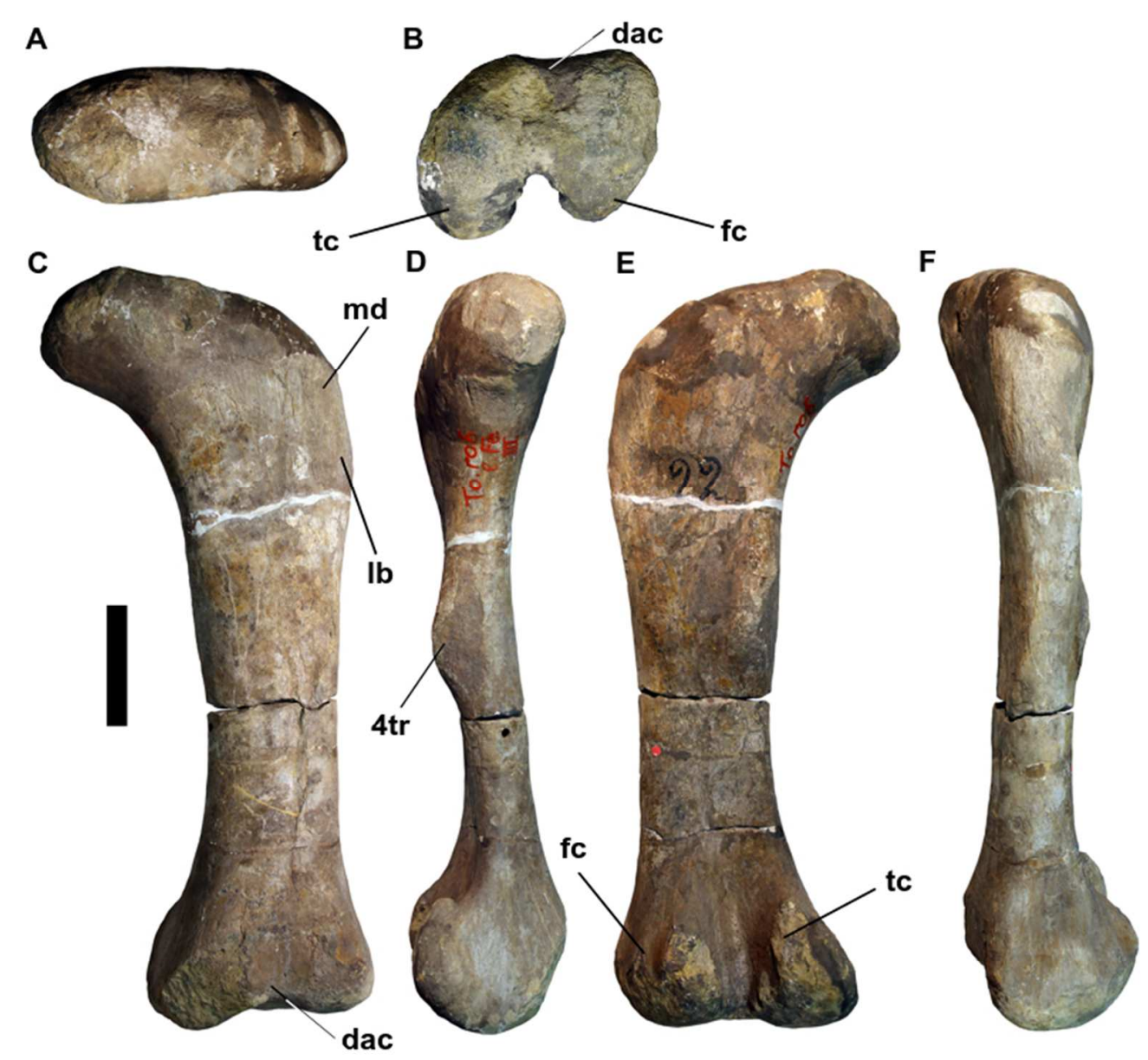



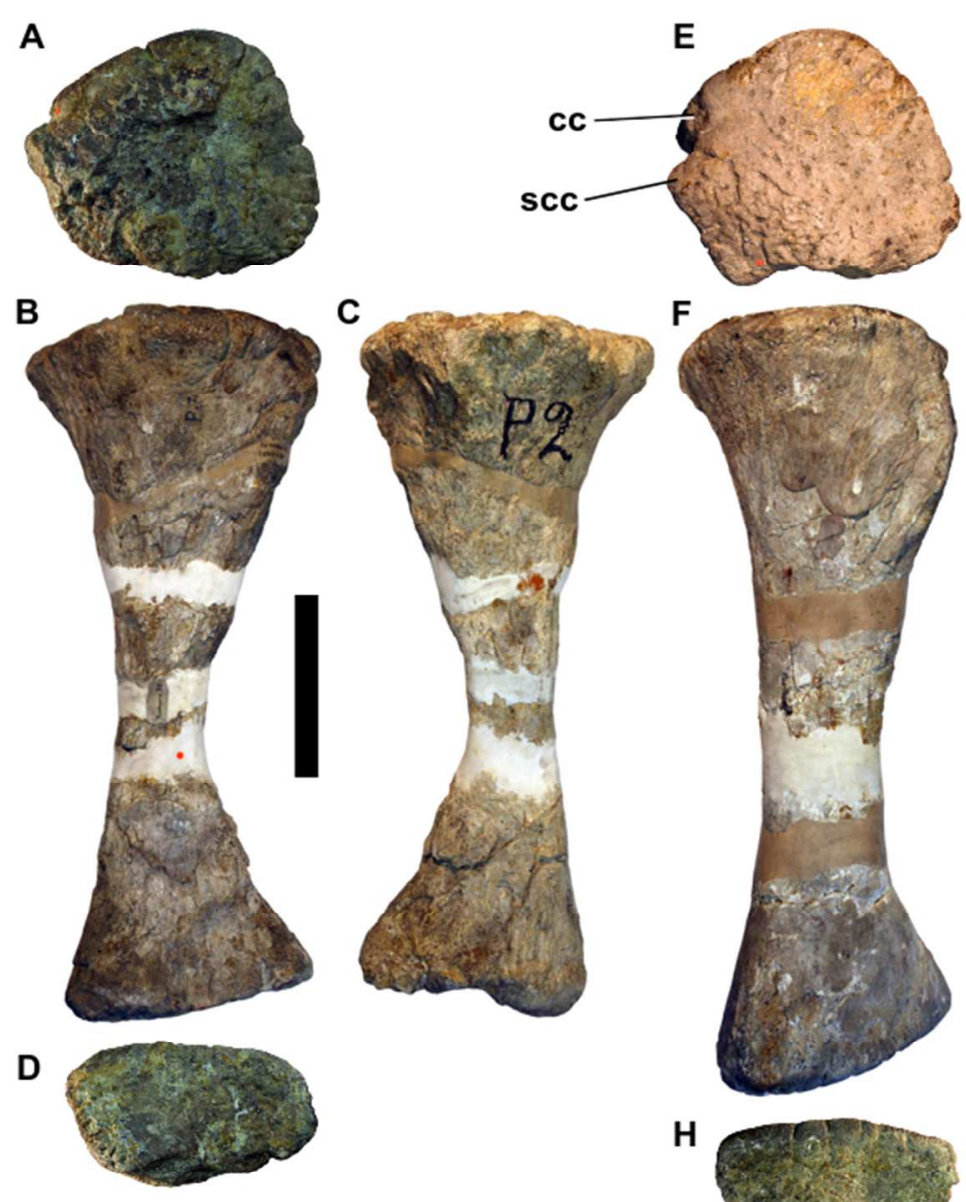

G

H
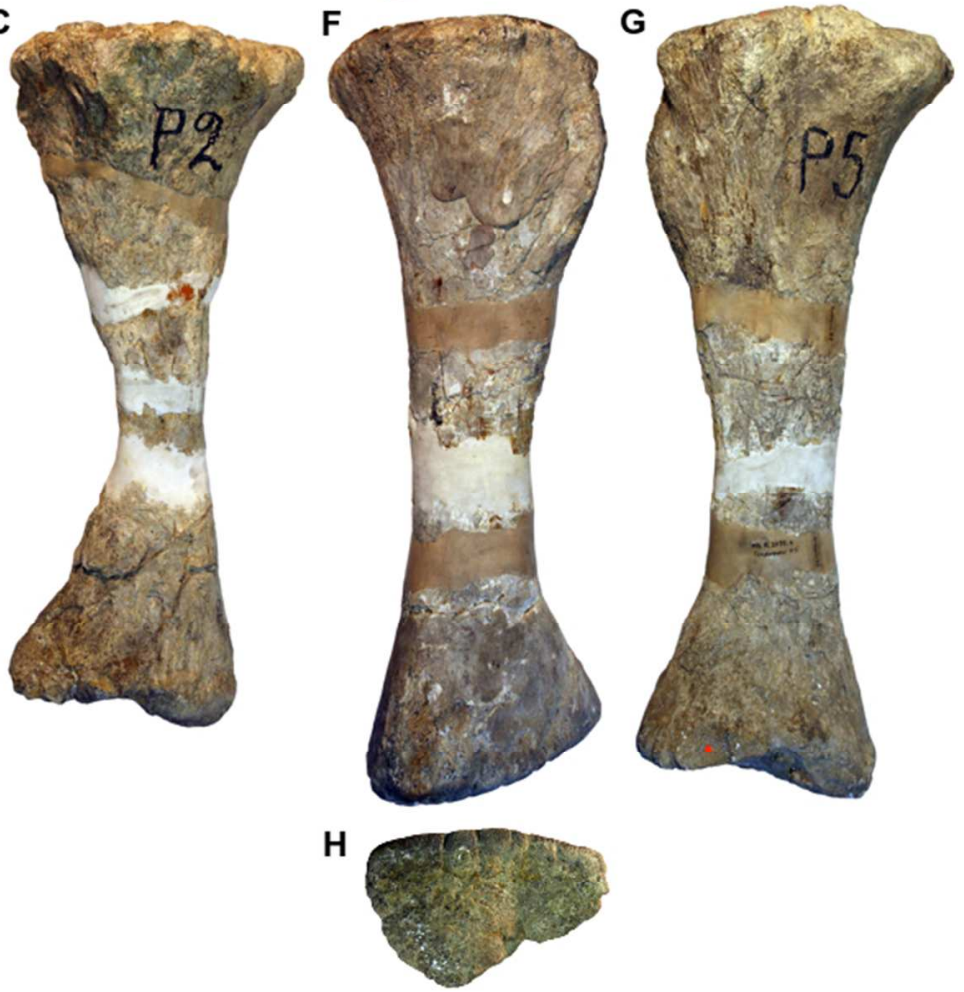


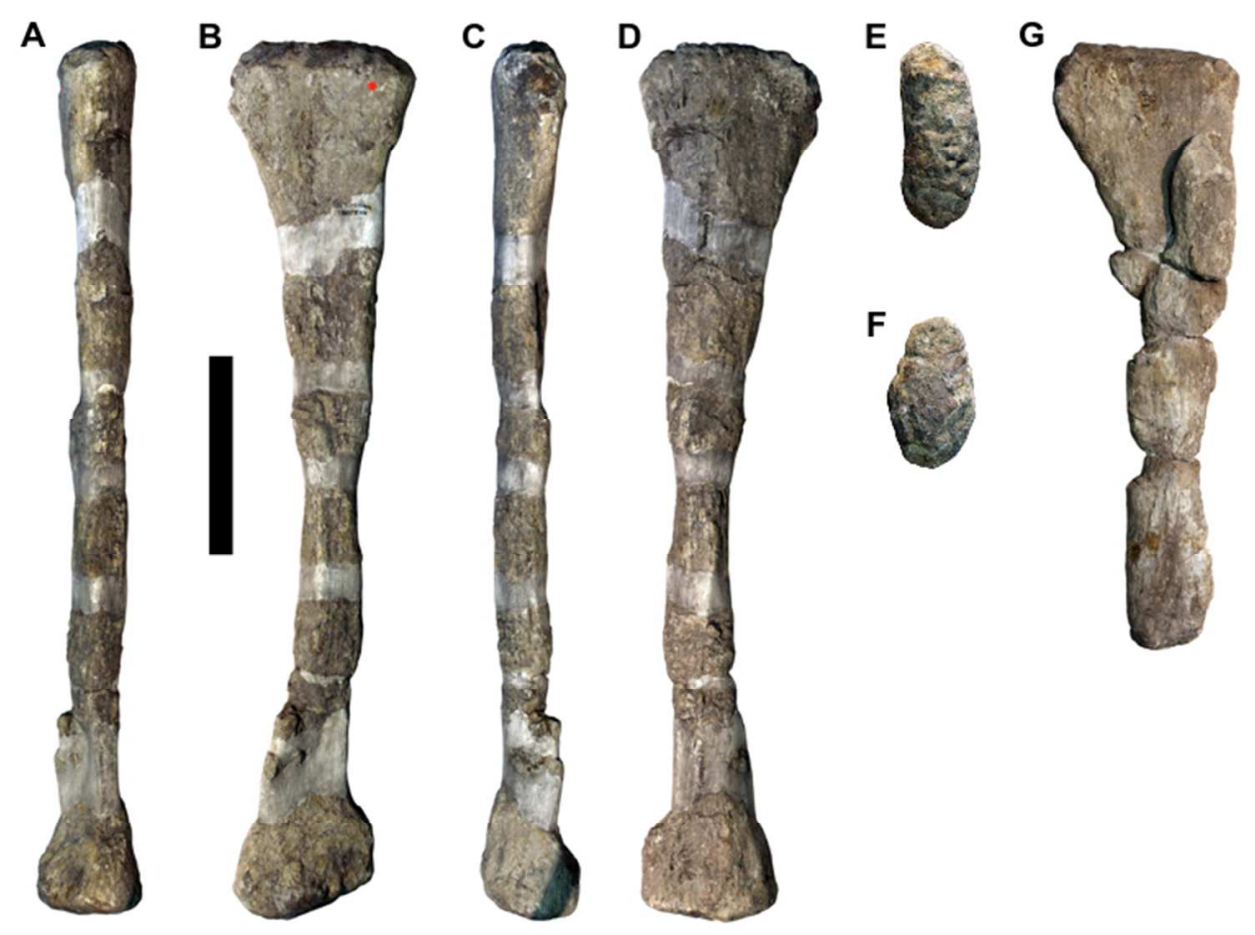


A

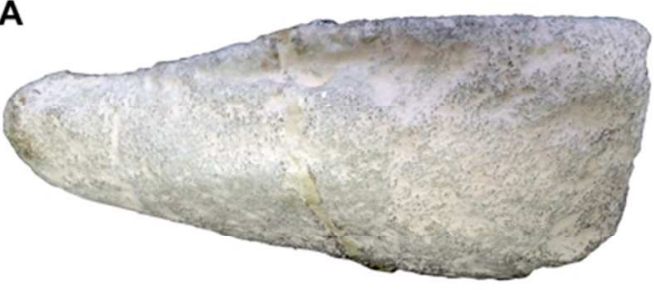

C
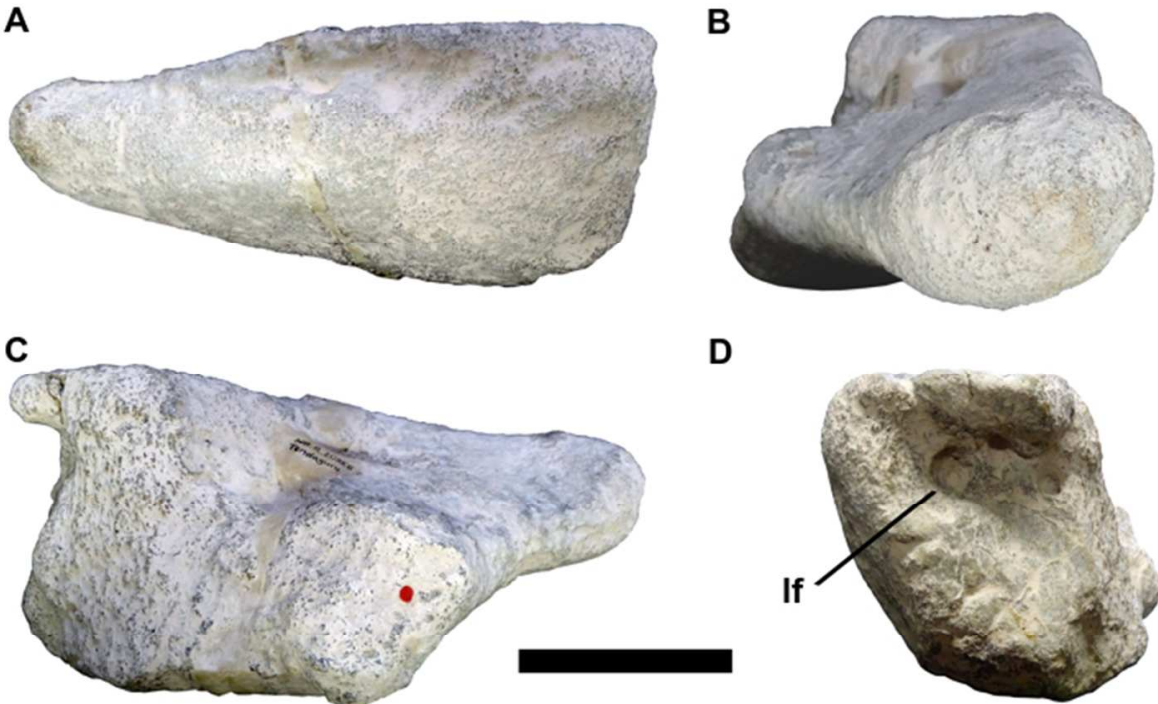

D

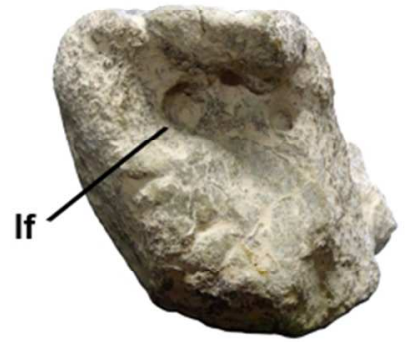

E
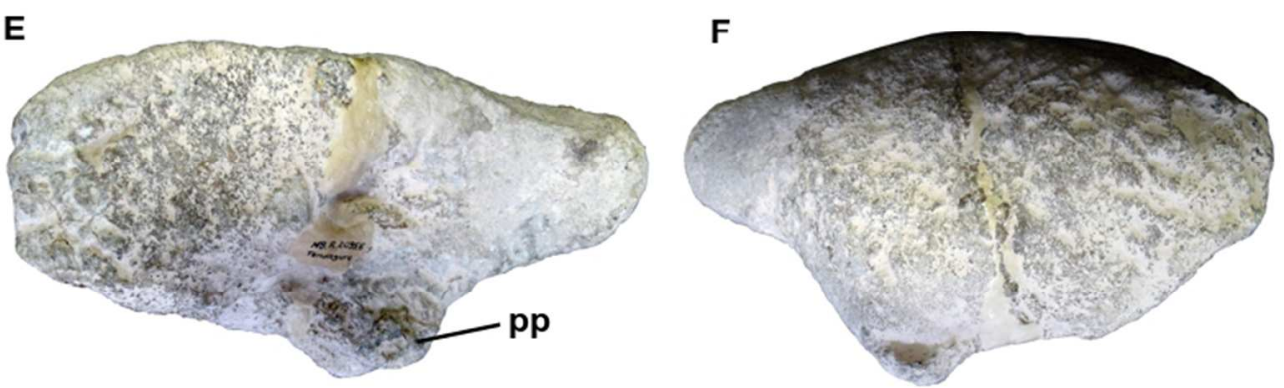

35 

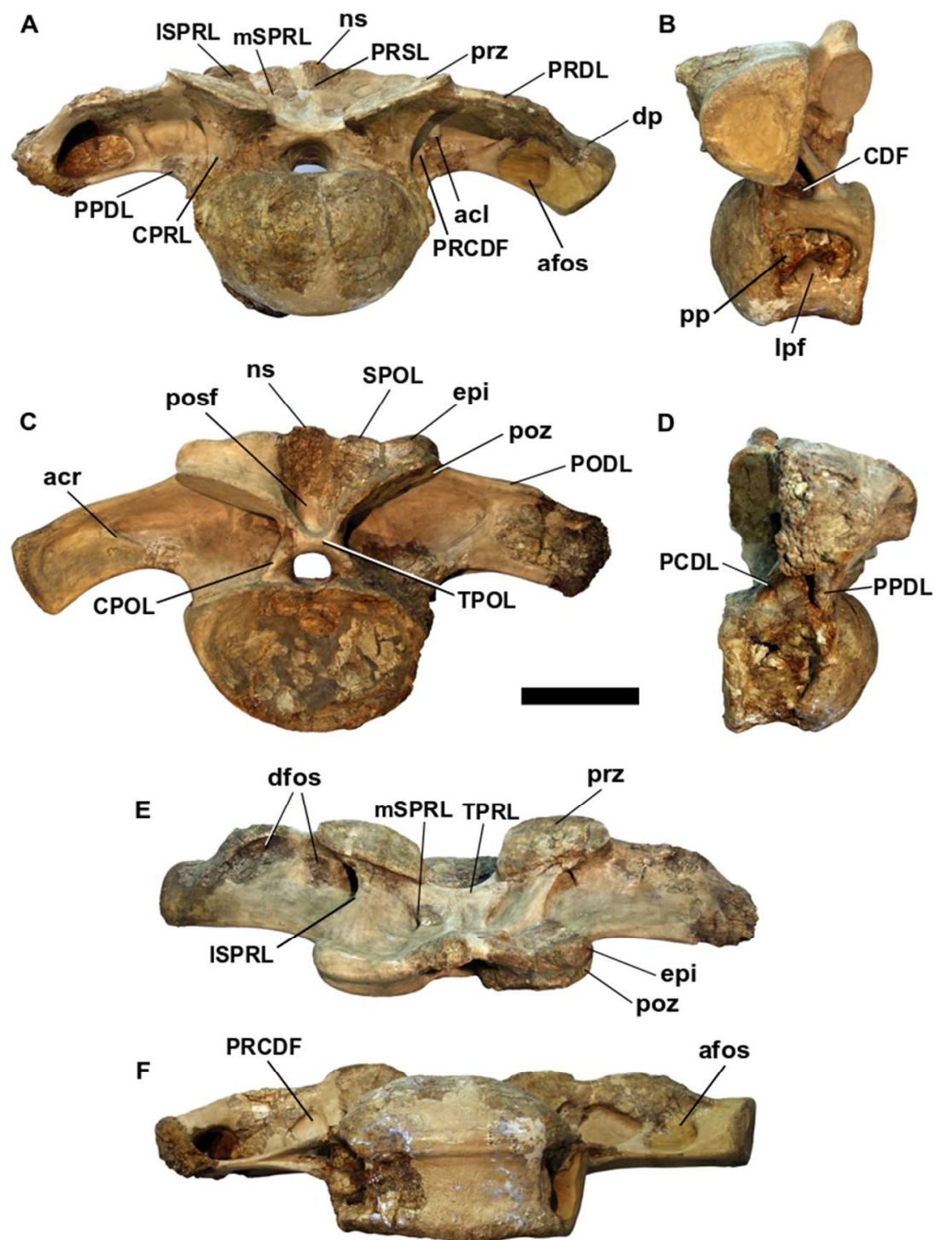

$280 \times 396 \mathrm{~mm}(72 \times 72 \mathrm{DPI})$ 

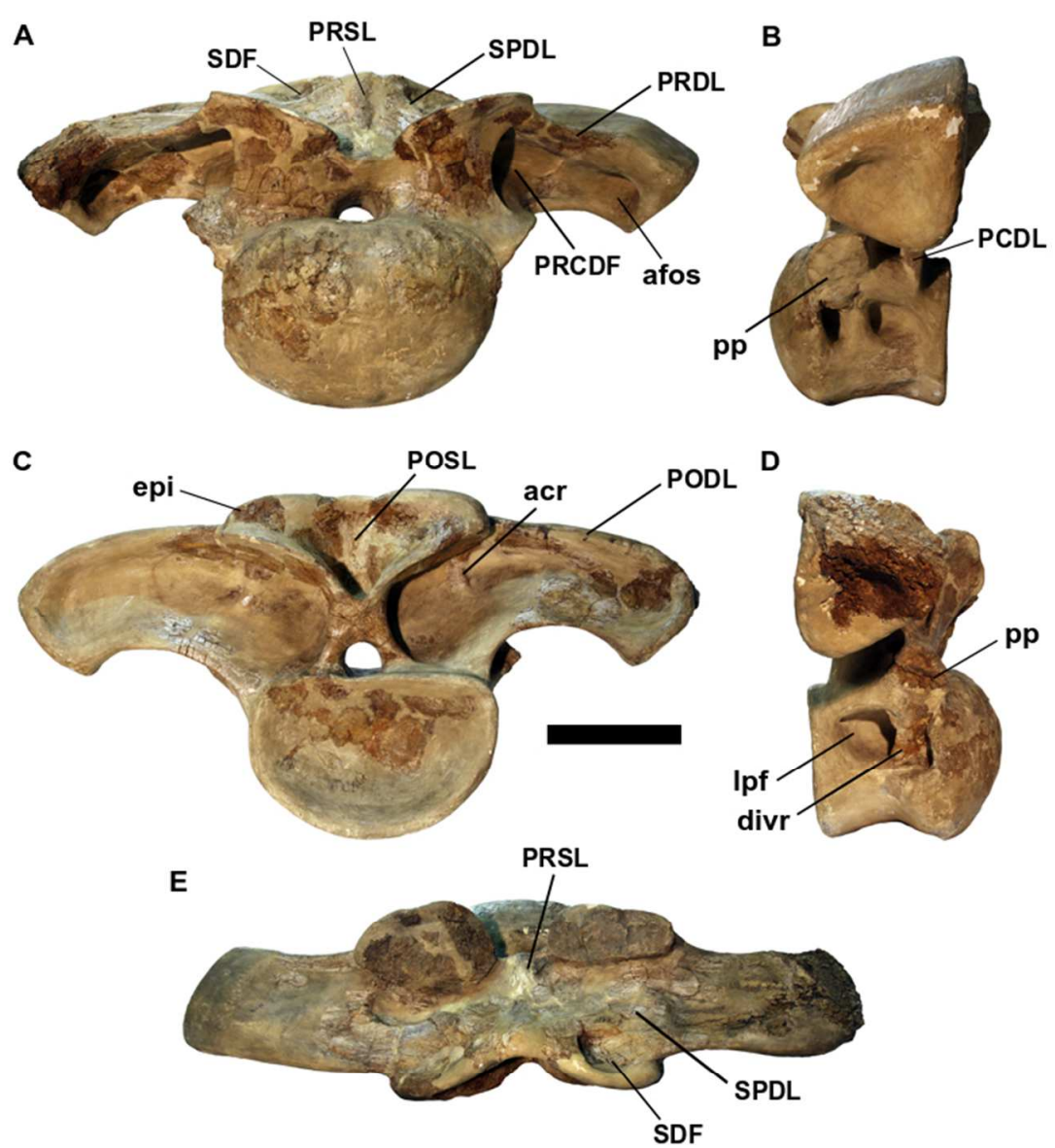

$\mathbf{F}$

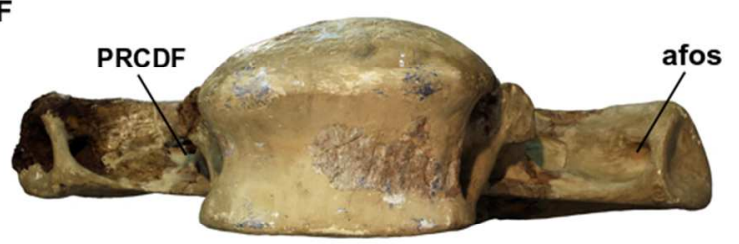

$280 \times 396 \mathrm{~mm}(72 \times 72 \mathrm{DPI})$ 
Figure 21. C-T scan images of Tendaguria tanzaniensis holotypic dorsal vertebrae (MB.R.2092.2 and 2092.1). Part A is a reconstruction of MB.R.2092.1 in left lateral view, showing the positions of transverse cross-sections that are illustrated in posterior view (Parts B-E). Part F is a reconstruction of MB.R.2092.2 in left lateral view, showing the positions of transverse cross-sections that are illustrated in posterior view (Parts G-M). Scale bars = $100 \mathrm{~mm}$ [intended for double column width]. 
A

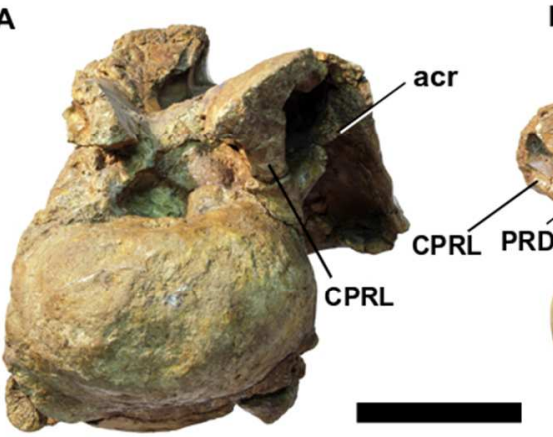

B

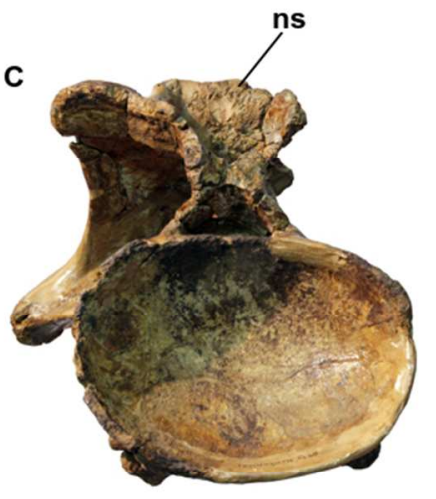

D

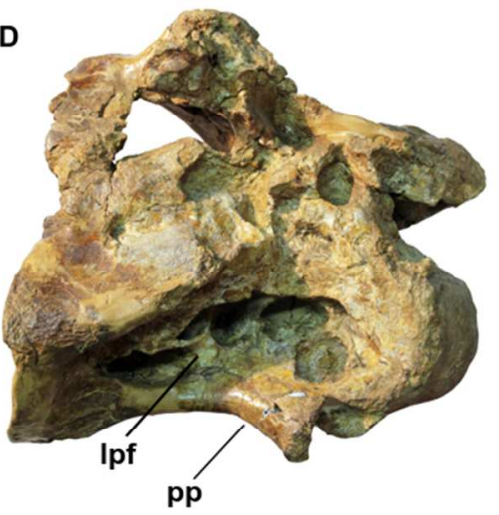

E

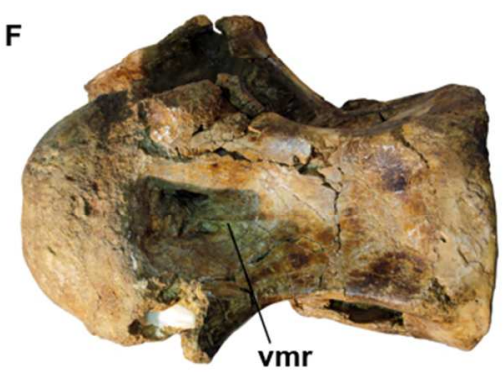

$280 \times 396 \mathrm{~mm}(72 \times 72$ DPI $)$ 
Figure 23. C-T scan images of cf. Tendaguria cervical vertebra (MB.R.2091.31). Part A is a reconstruction of MB.R.2091.31 in left lateral view, showing the positions of transverse cross-sections that are illustrated in posterior view (Parts B-L). Part M shows a medial cross-section, and parts N-P show horizontal crosssections illustrated in dorsal view. Scale bars $=50 \mathrm{~mm}$ [intended for double column width]. 

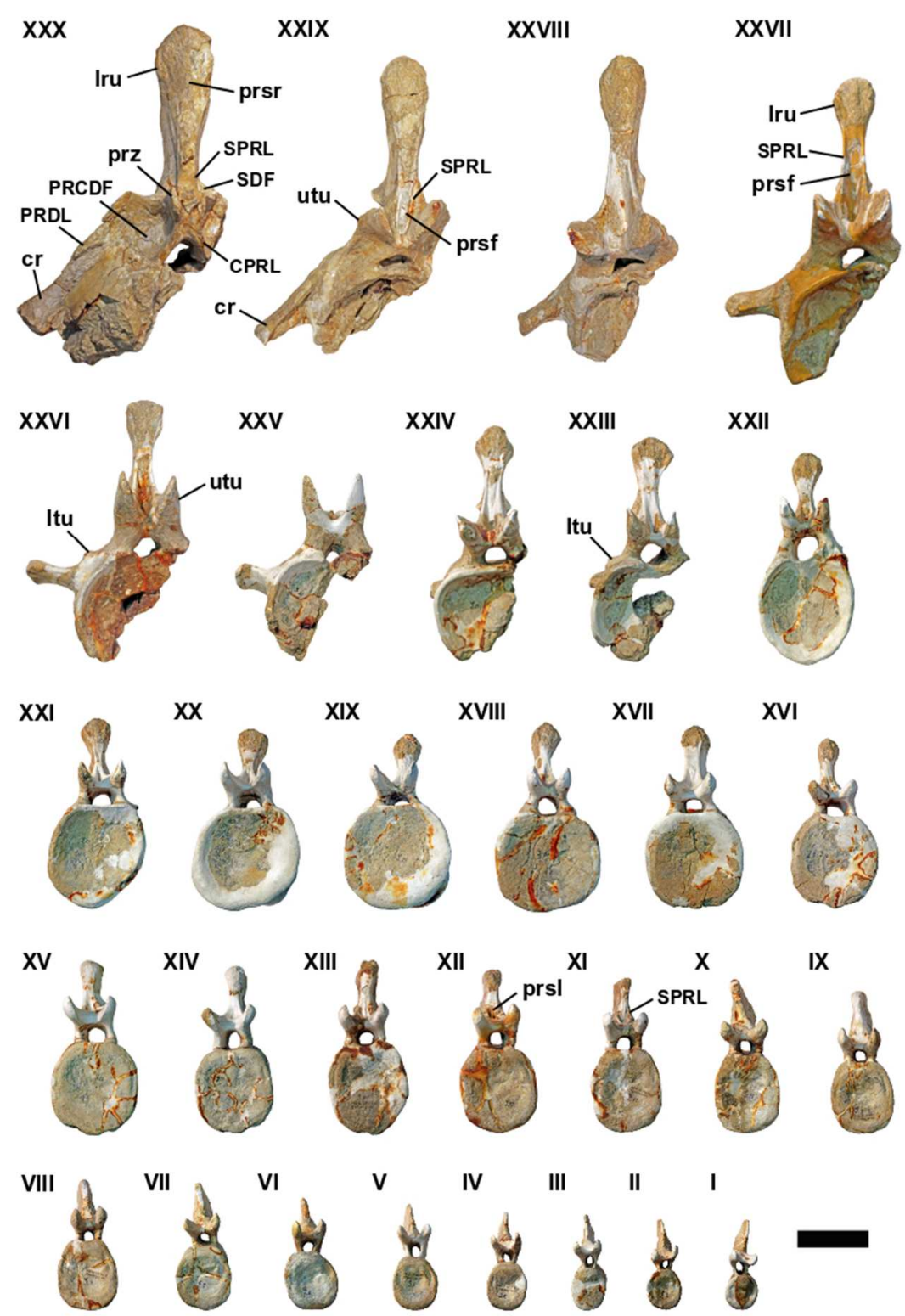
$280 \times 396 \mathrm{~mm}(72 \times 72$ DPI $)$ 

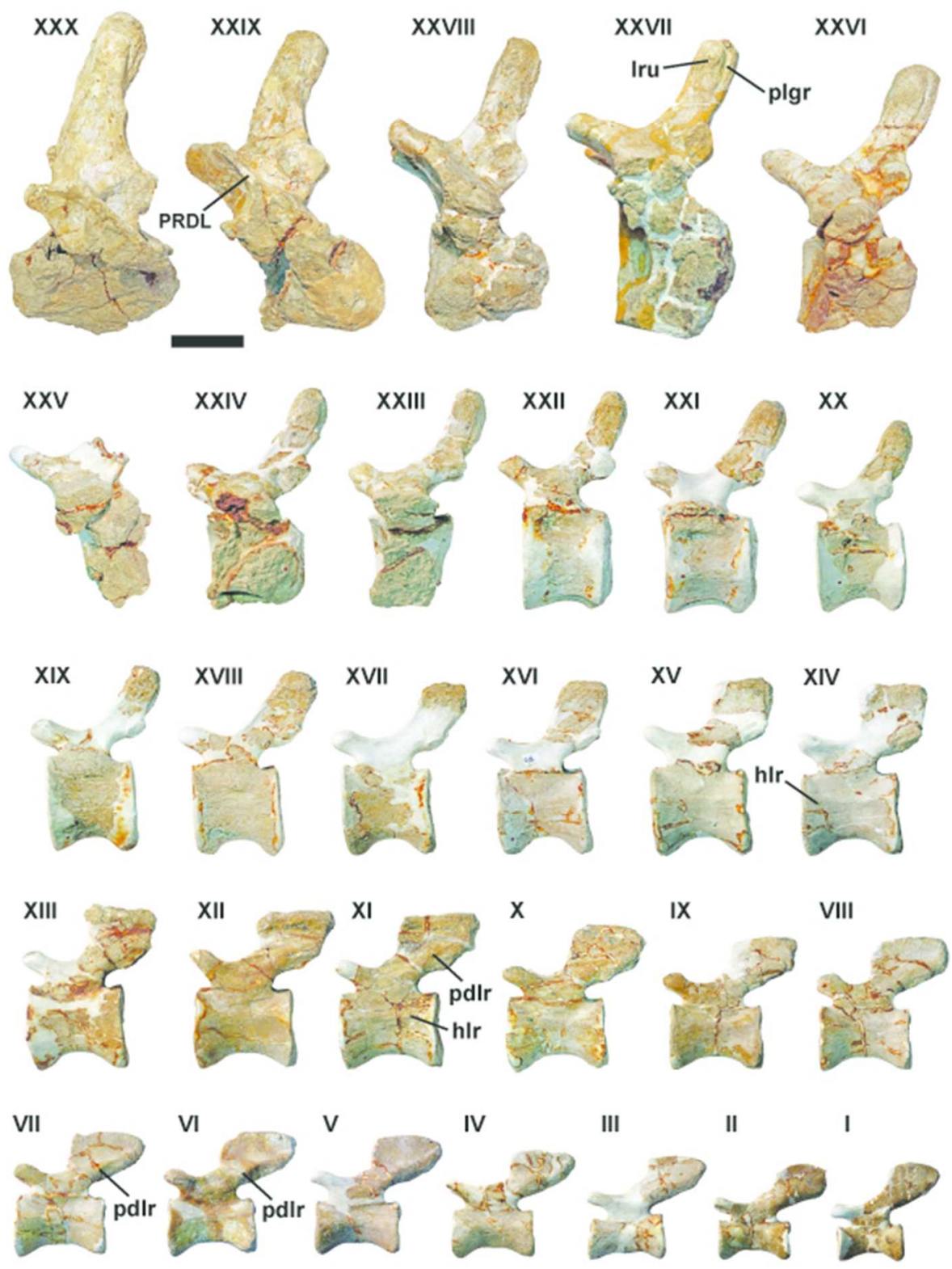

$207 \times 275 \mathrm{~mm}(72 \times 72$ DPI $)$ 

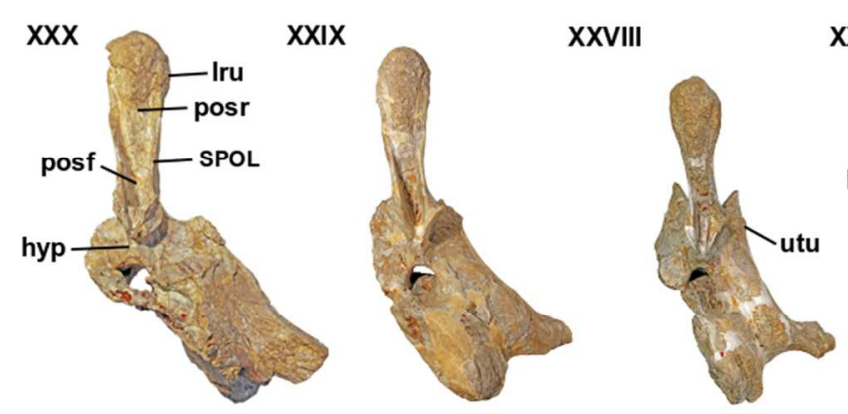

XXVII

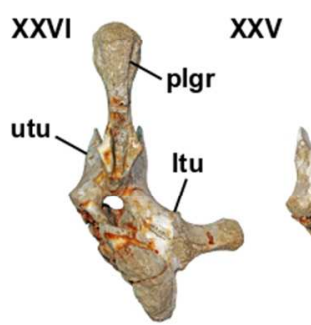

XXIV

XXIII
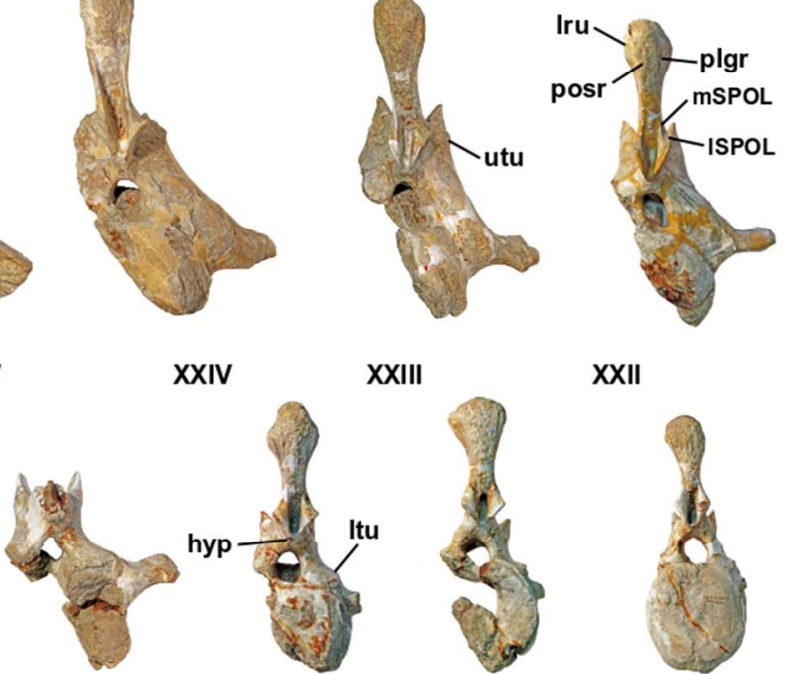

XXII

XXI

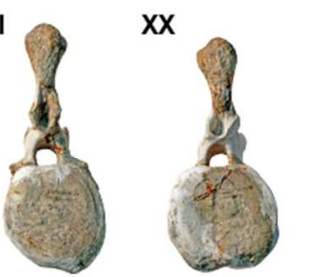

XIX

XVIII

XVII
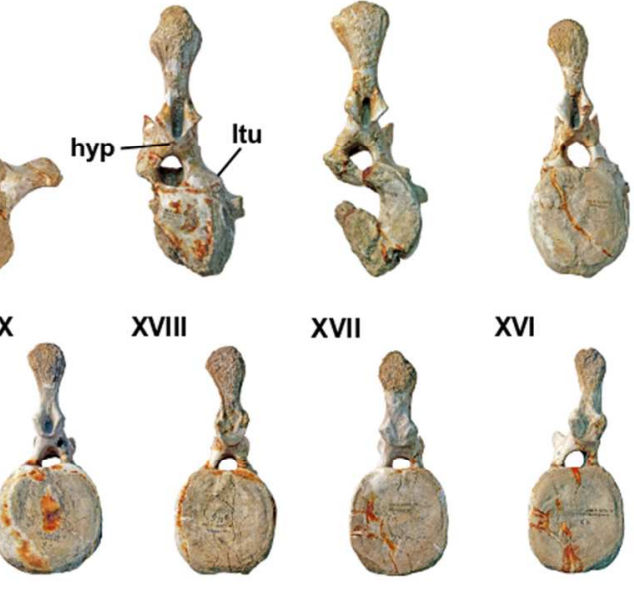

XVI

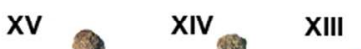

XII

XI

$\mathrm{x}$
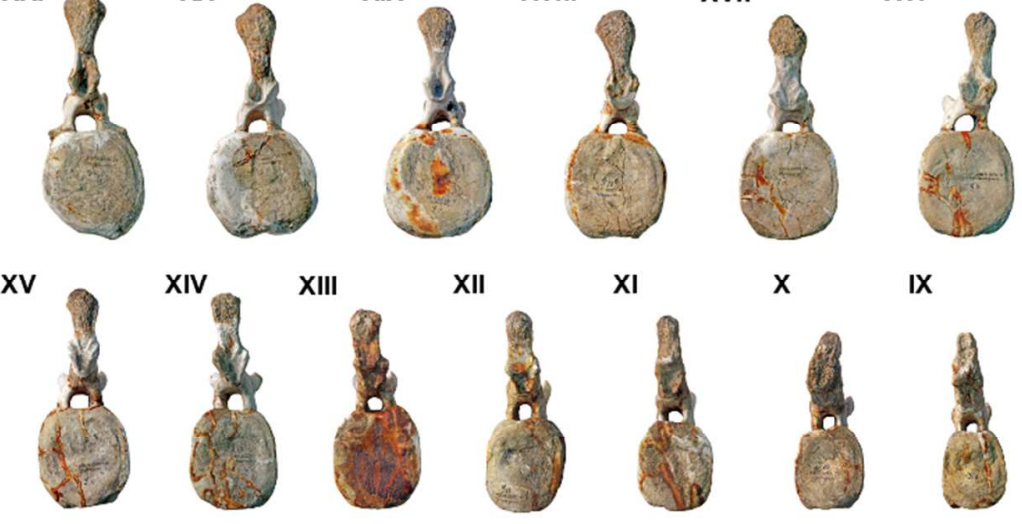

IX

u'm

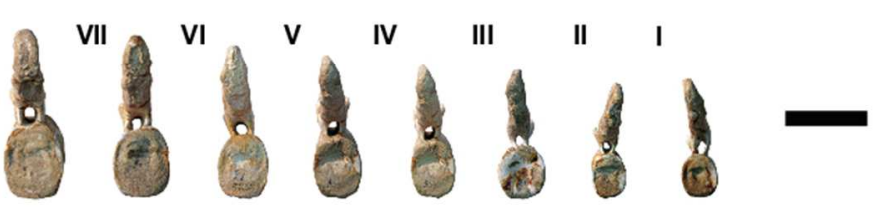

$280 \times 396 \mathrm{~mm}(72 \times 72 \mathrm{DPI})$ 

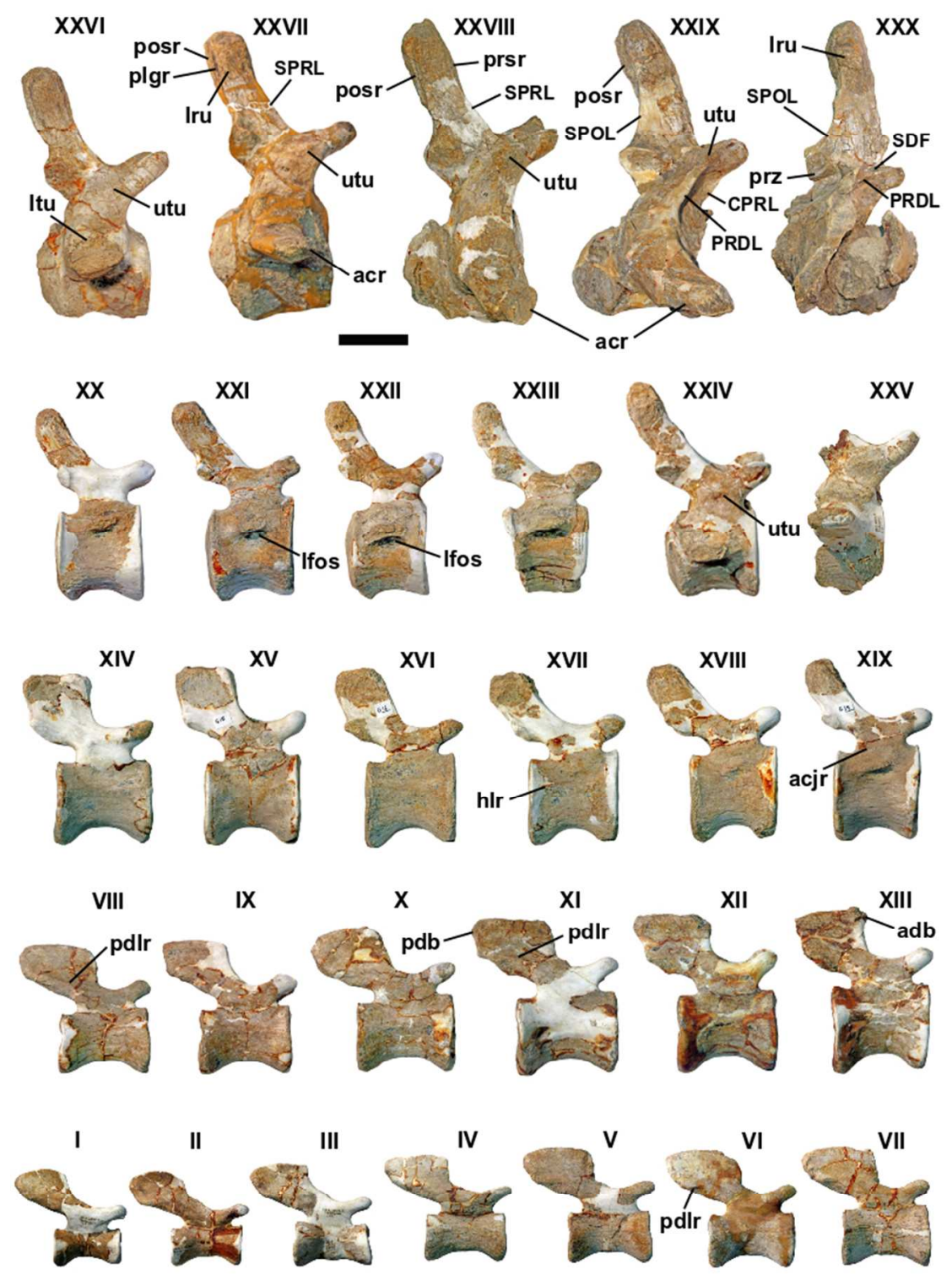

$280 \times 396 \mathrm{~mm}(72 \times 72 \mathrm{DPI})$ 

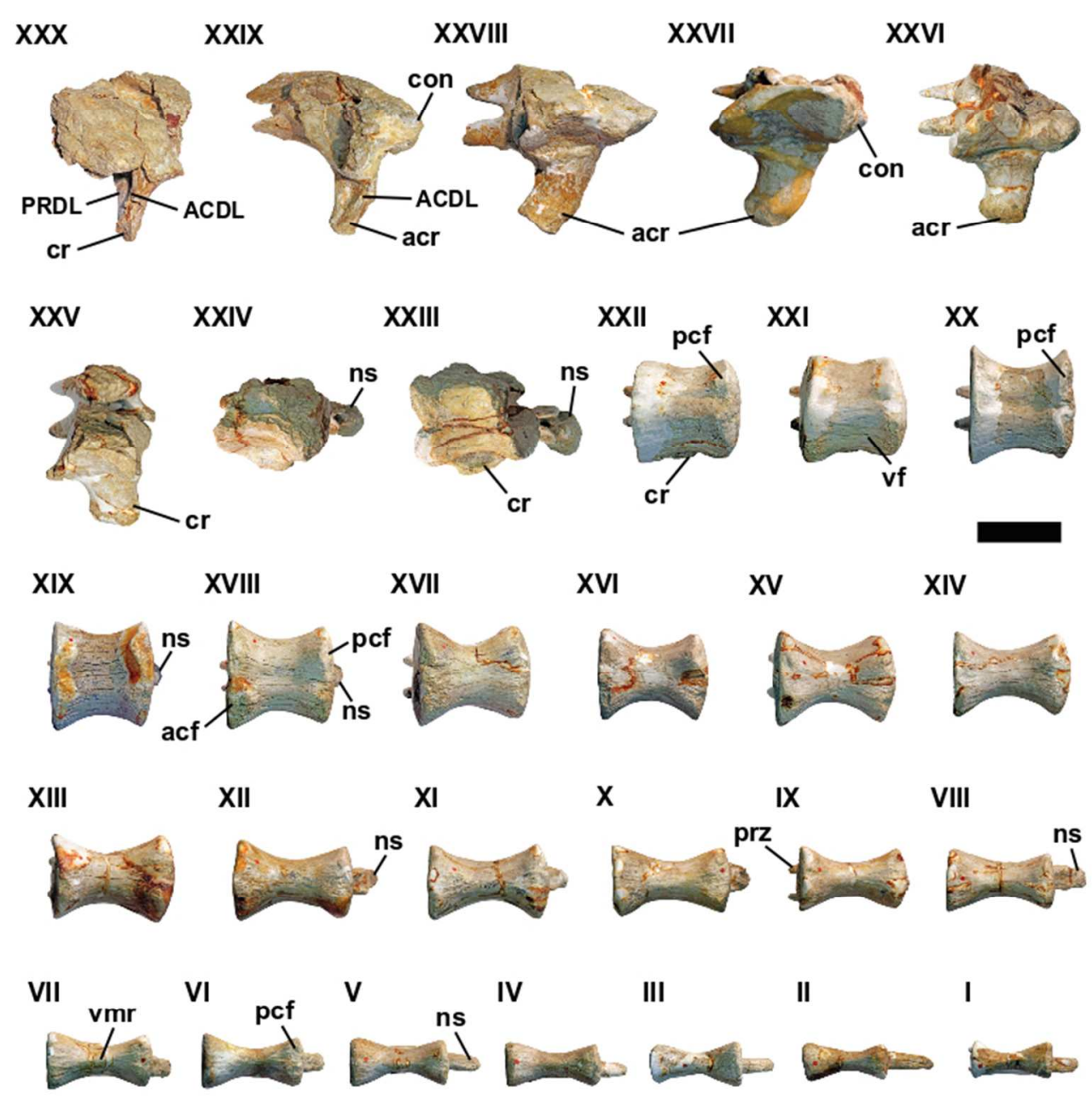
Zoological Journal of the Linnean Society

Page 170 of 186

1
2
3
4
5
6
7
8
9
10
11
12
13
14
15
16
17
18
19
20
21
22
23
24
25
26
27
28
29
30
31
32
33
34
35
36
37
38
39
40
41
42
43
44
45
46
47
48
49
50
51
52
53
54
55
56
57
58
59
60 
解苚以 
A

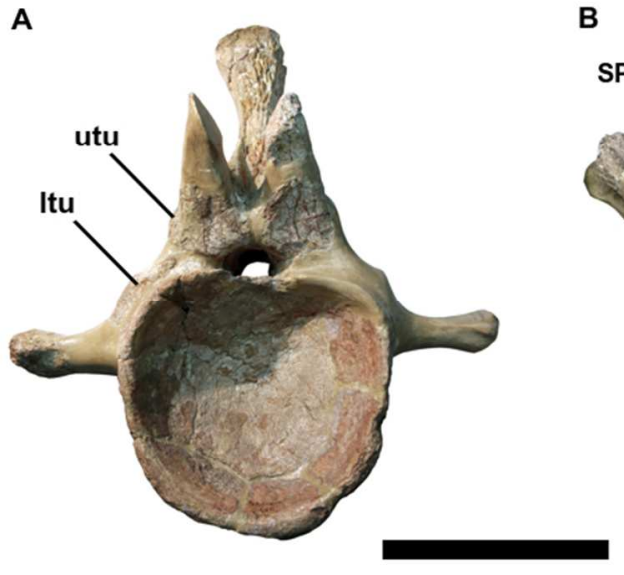

C
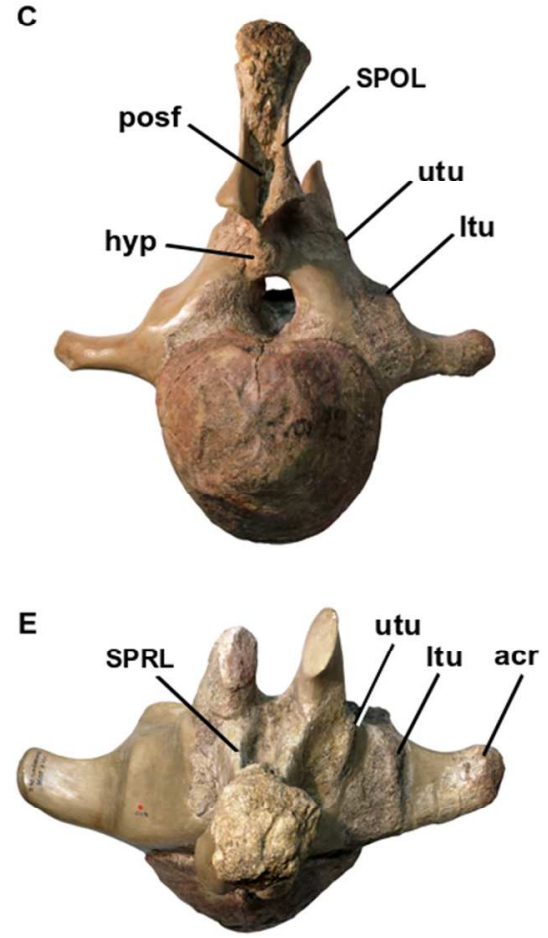

B

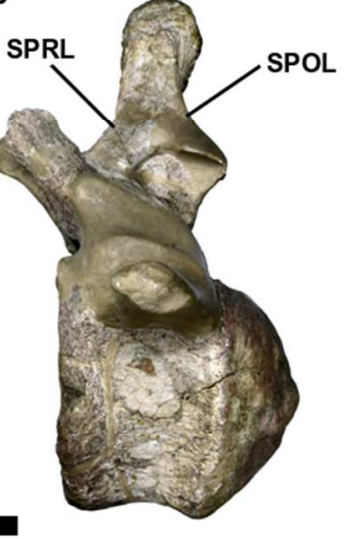

D

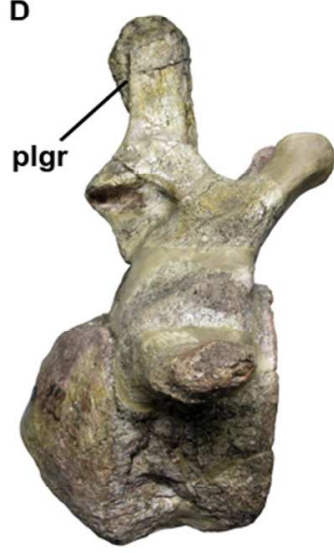

F

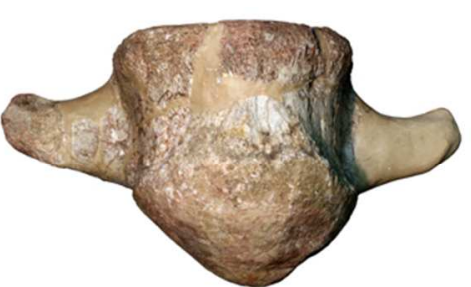
$280 \times 396 \mathrm{~mm}(72 \times 72 \mathrm{DPI})$ 

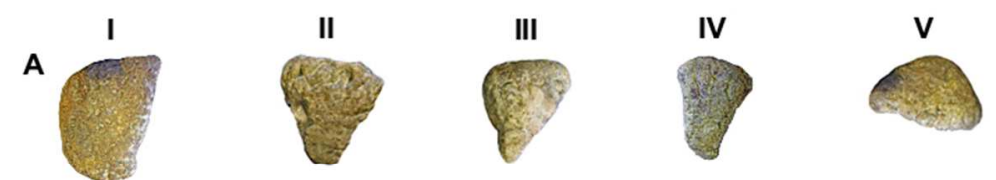

B
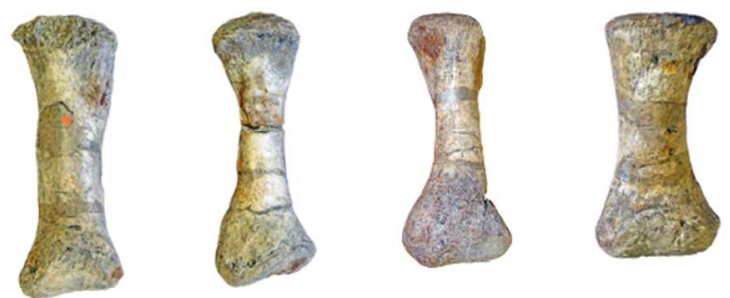

C
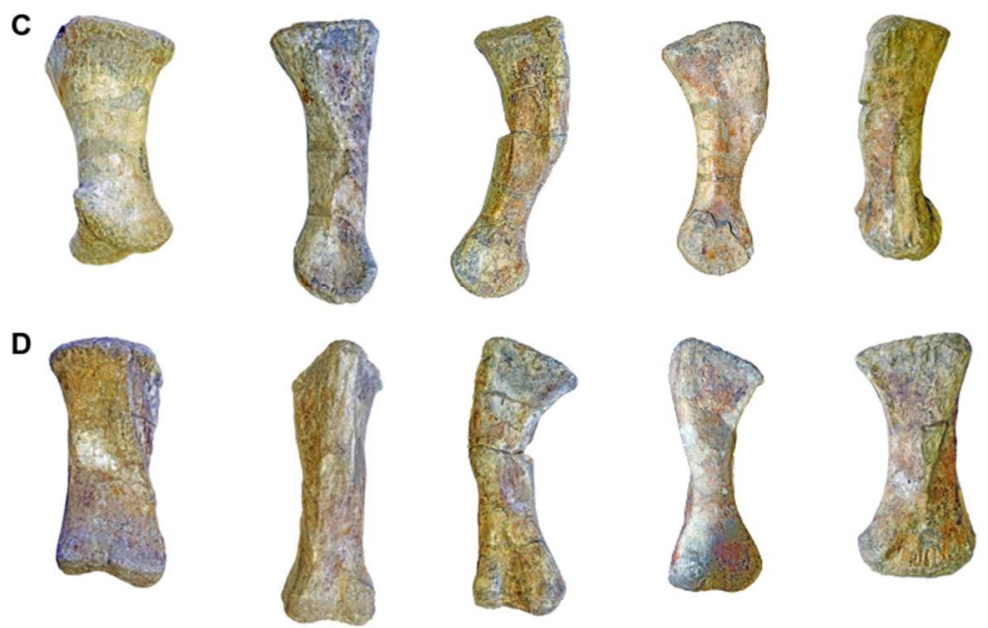

E
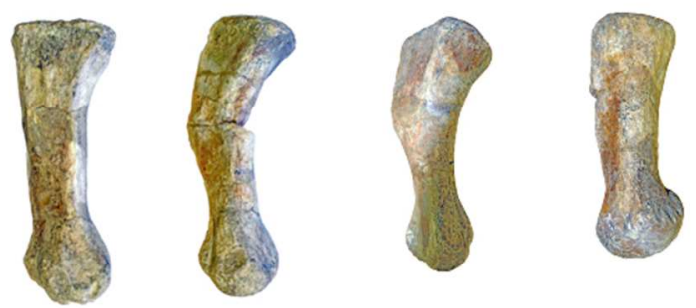

F
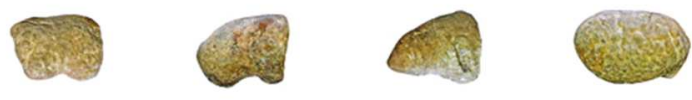

$280 \times 396 \mathrm{~mm}(72 \times 72$ DPI $)$ 


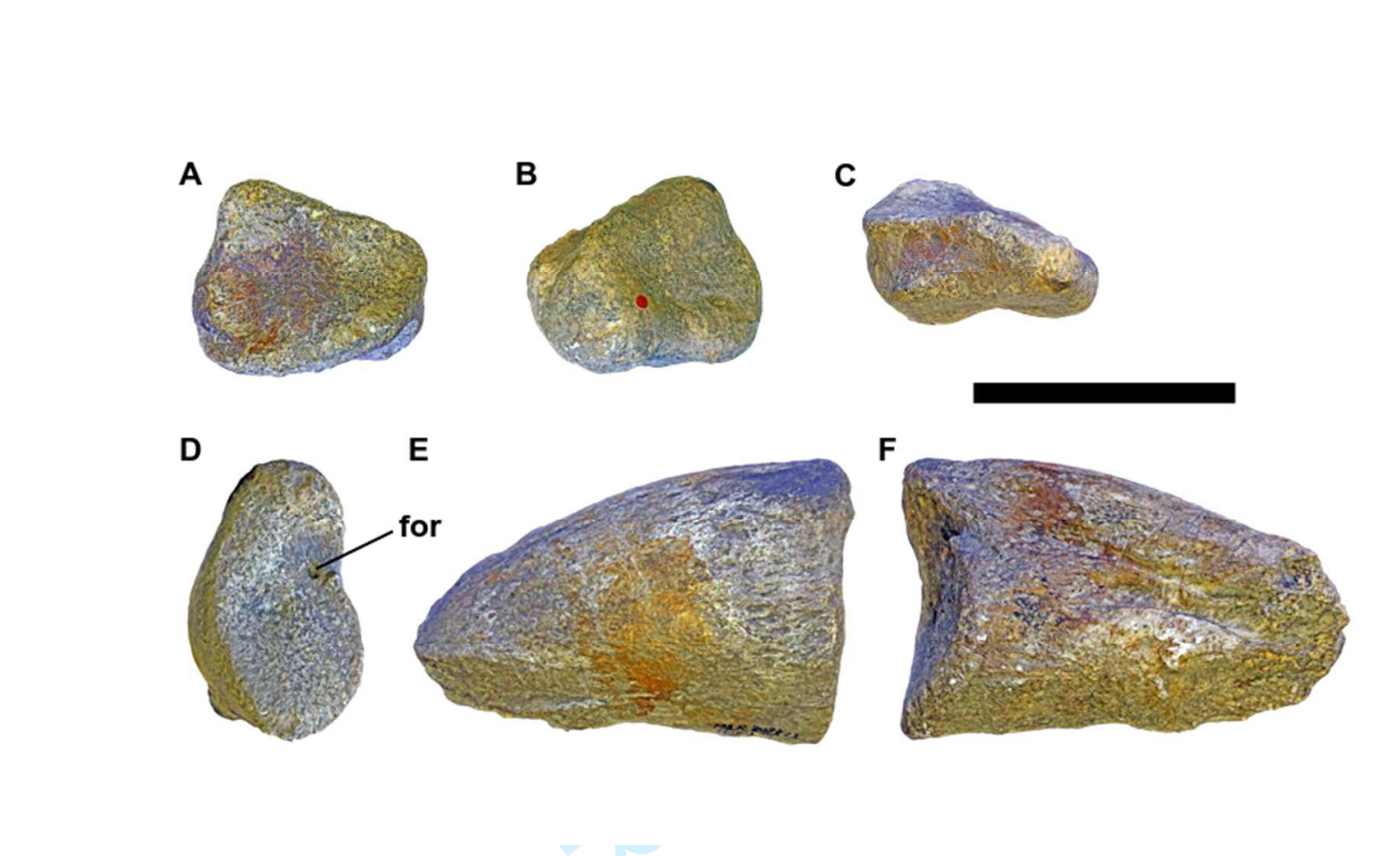




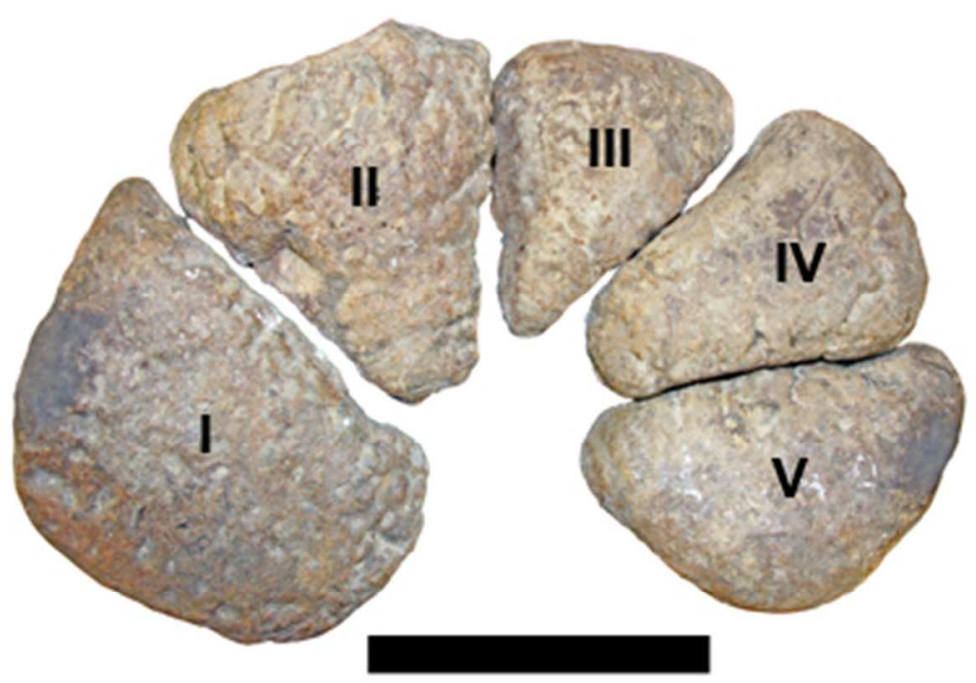



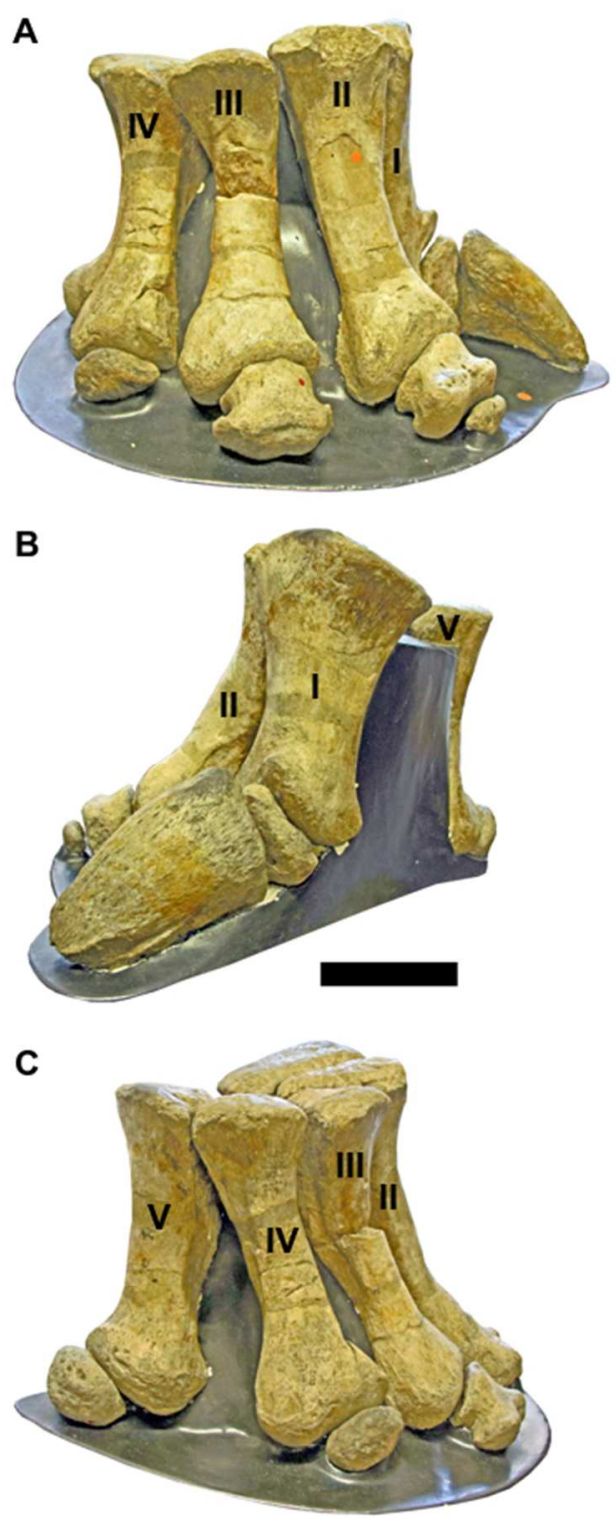


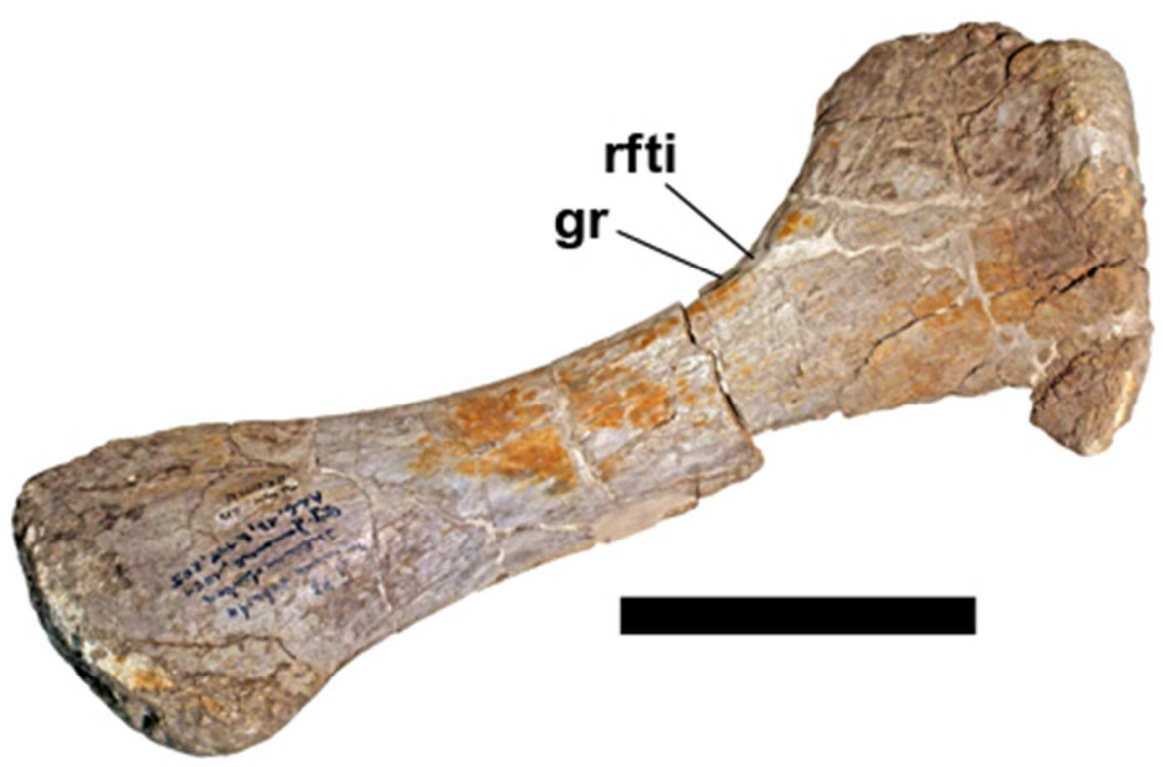




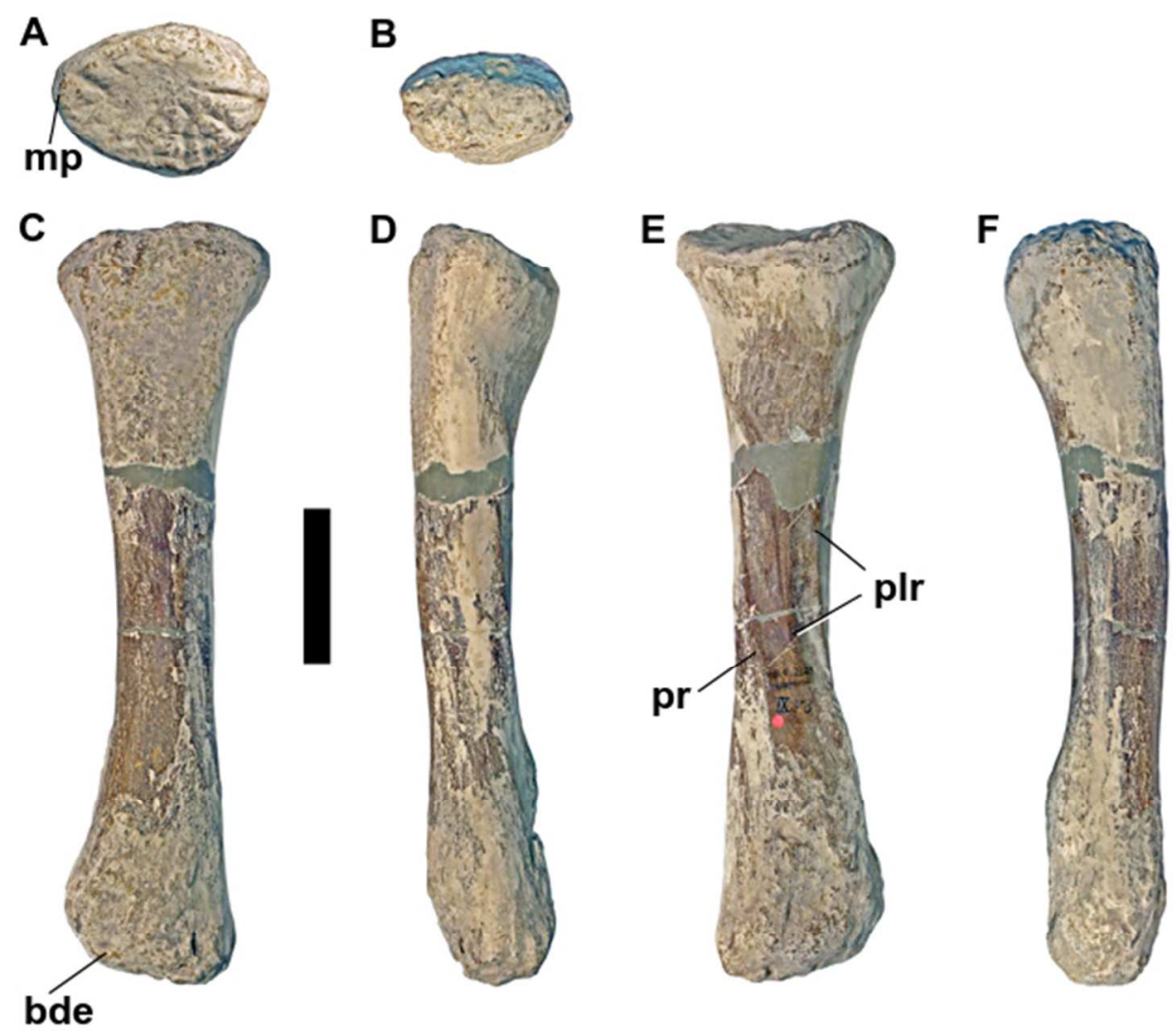



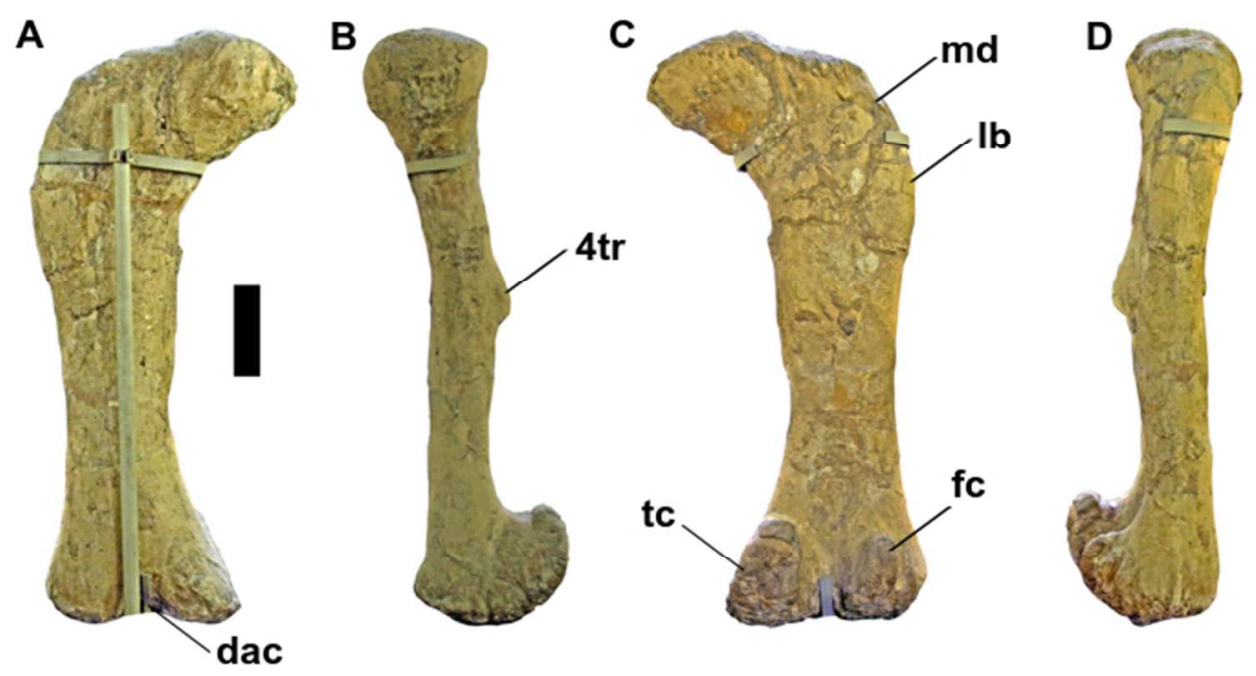


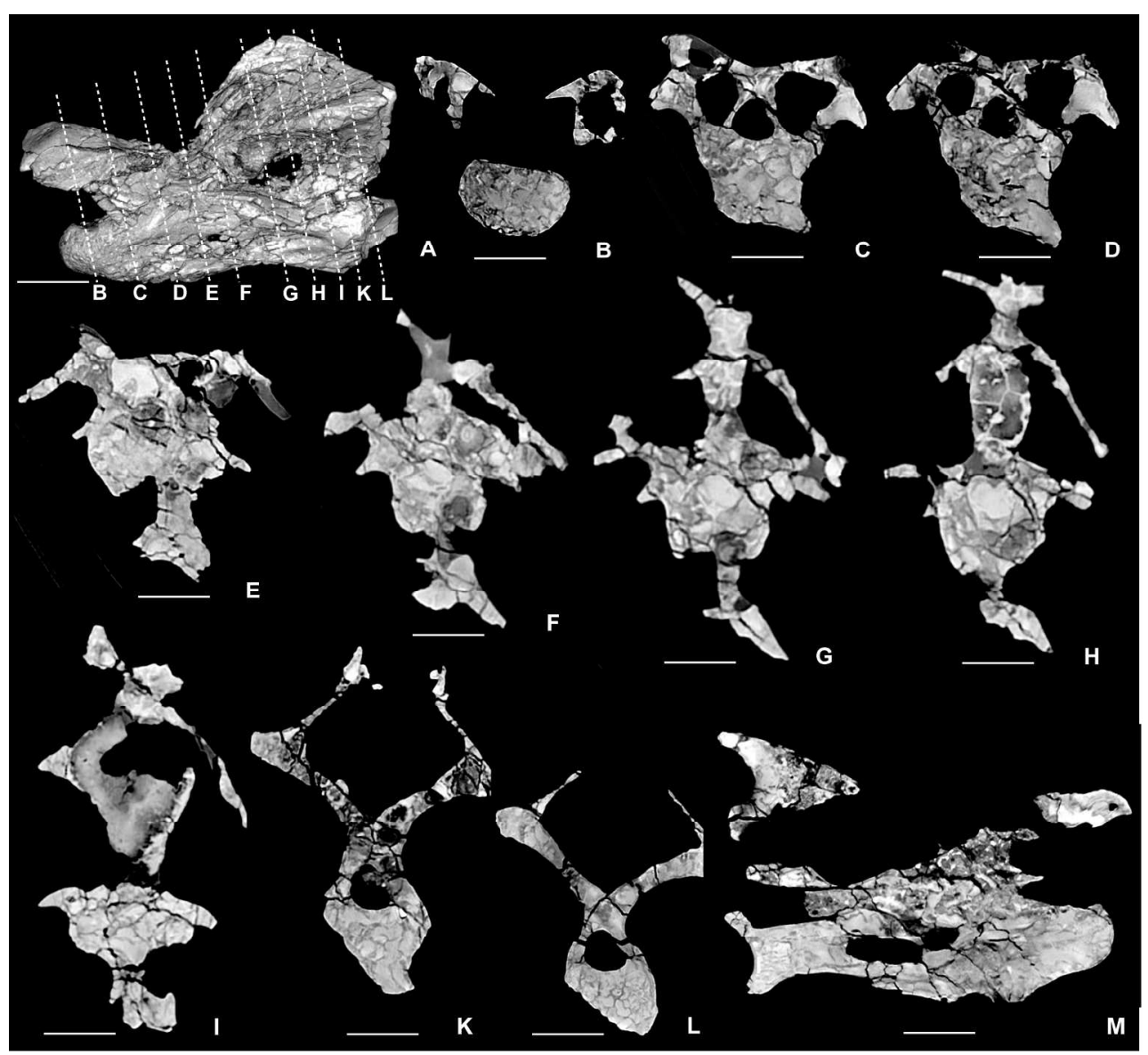

Figure 39. C-T scan images of Australodocus bohetii holotypic cervical vertebra (MB.R.2455). Part A is a 3D reconstruction of the vertebra in left lateral view, showing the positions of transverse cross-sections that are illustrated in posterior view (Parts B-L). Part M shows a medial, longitudinal cross-section, viewed from the right side of the vertebra. Scale bars $=50 \mathrm{~mm}$ [intended for double column width]. 


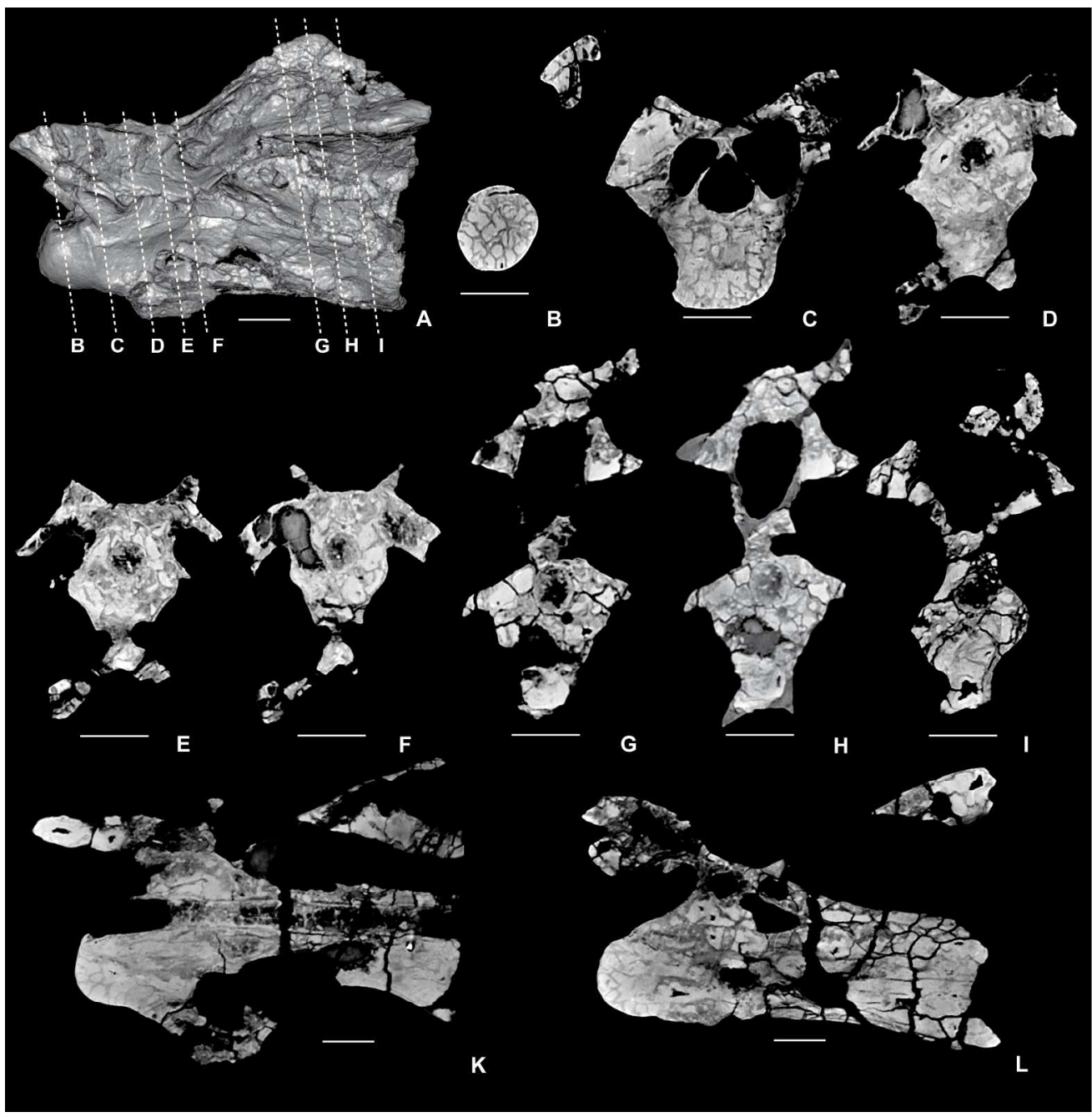

Figure 40. C-T scan images of Australodocus bohetii paratypic cervical vertebra (MB.R.2454) Part A is a 3D reconstruction of the vertebra in left lateral view, showing the positions of transverse cross-sections that are illustrated in posterior view (Parts B-I). Parts K and L show longitudinal cross-sections, viewed from the left side of the vertebra. Scale bars $=50 \mathrm{~mm}$ [intended for double column width].

$$
210 \times 213 \mathrm{~mm}(300 \times 300 \text { DPI) }
$$



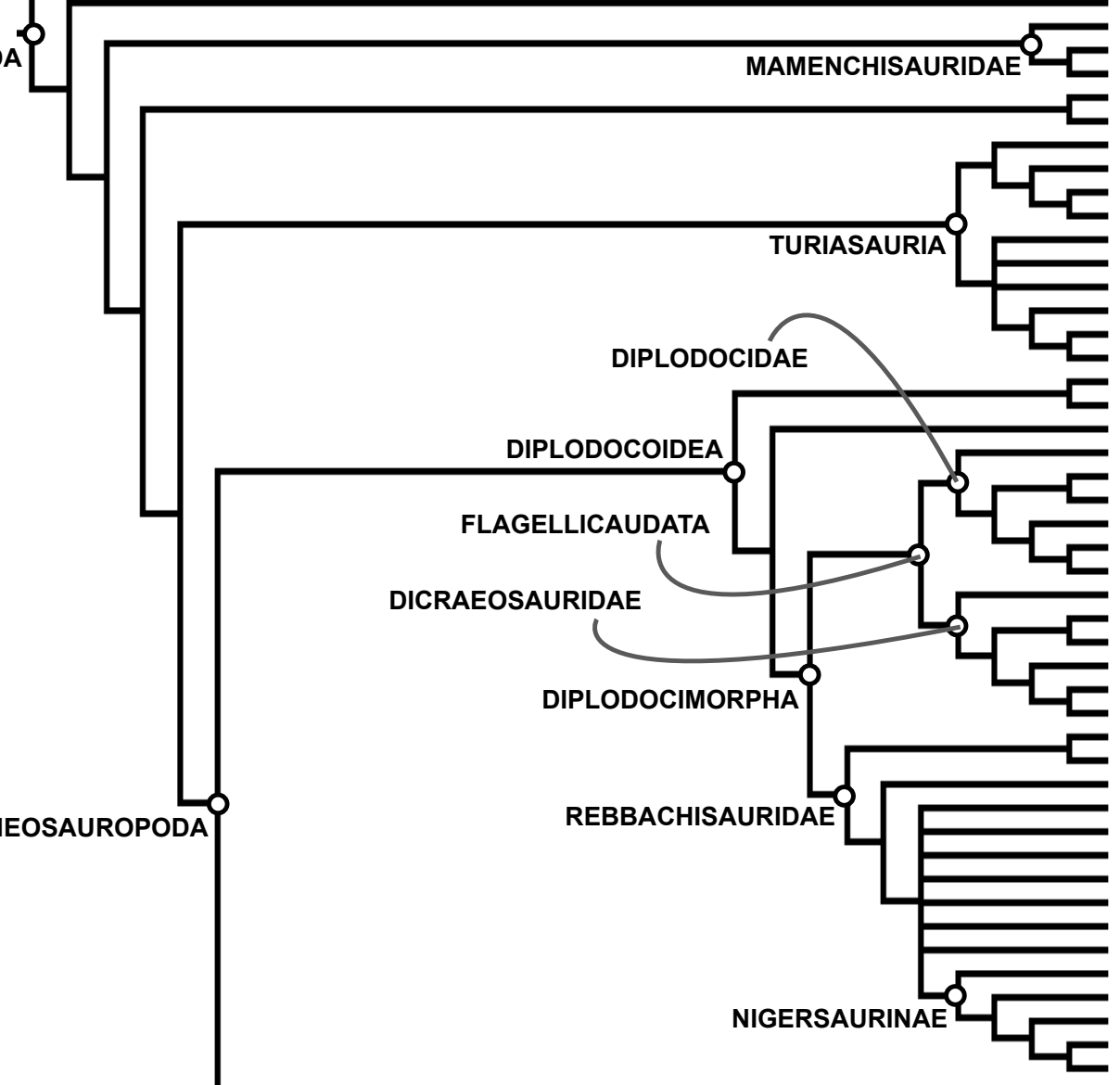

Chuanjiesaurus Mamenchisaurus Wamweracaudia Jobaria

Lapparentosaurus

Tehuelchesaurus

Bellusaurus

Haestasaurus

Janenschia

Losillasaurus

Turiasaurus

Zby

Mierasaurus

Moabosaurus

Tendaguria

Haplocanthosaurus priscus

Haplocanthosaur

Apatosaurus

Dinheirosaurus

Supersaurus

Diplodocus

Leinkupal

Tornieria

Suuwassea

Lingwulong

Amargasaurus

Amargasaurus

Brachytrachelopan

Dicraeosaurus

Amazonsaurus

Histriasaurus

NEOSAUROPODA

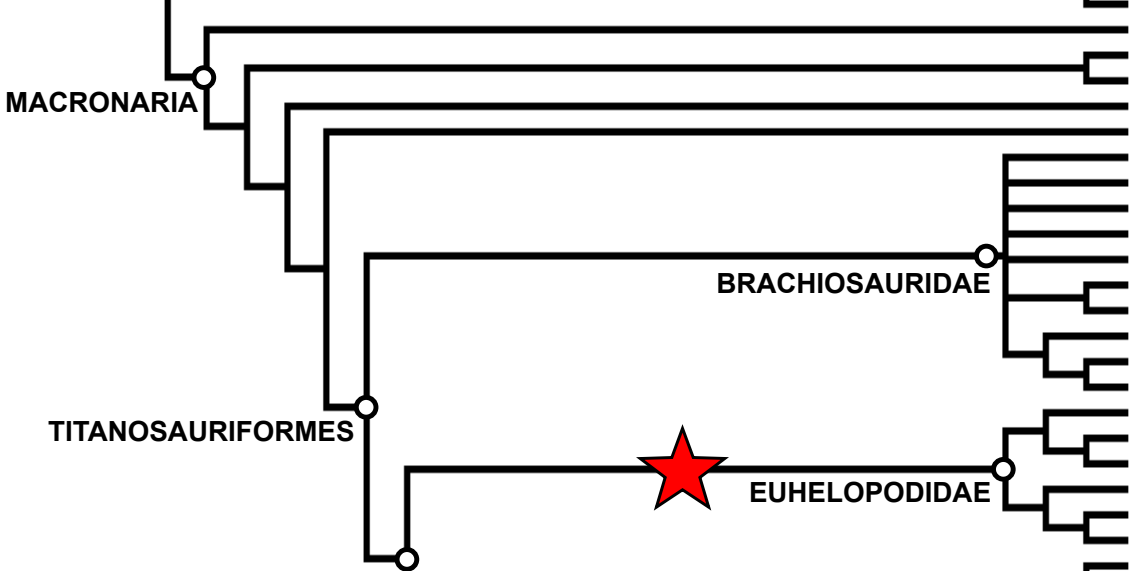

Atlasaurus

Atlasaurus

El Chocon rebbachisaurid

Katepensaurus

Limaysaurus

Nopcsaspondylus

Rayososaurus

Zapalasaurus

Nigersaurus

Rebbachisaurus

ataouinea

Camarasaurus

Aragosaurus

Galveosaurus

Abydosaurus

Cedarosaurus

Europasaurus

Soriatitan

Venenosaurus

Lusotitan

Vouivria

Brachiosaurus

Giraffatitan

Sonorasaurus

Euhelopus

\section{Erketu}

\section{Gobititan}

Qiaowanlong

Phuwiangosaurus

Tangvayosaurus

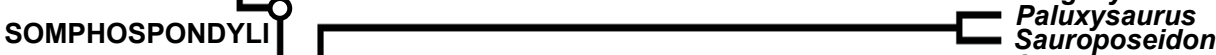

Sauroposeidon

Cloverly titanosauriform

Padillasaurus

Dongbeititan

Ligabuesaurus

Angolatitan

Angolatitan

Chubutisaurus

Baotianmansaurus

Ruyangosaurus

Wintonotitan

Huanghetitan liujiaxiaensis

Huanghetitan rujla

Andesaurus

Andesaurus

Malawisaurus

Muyelensaurus

Pitekunsaurus

Epachthosaurus

Argentinosaurus

Daxiatitan

LITHOSTROTIA

Dongyangosaurus

Futalognkosaurus

Mendozasaurus

Mongolosaurus

Nemegtosaurus

Notocolossus

Patagotitan

Puertasaurus

Puertasaurus

Tapuiasaurus

AODF 836

AODF 836

Diamantinasaurus

Savannasaurus

Opisthocoelicaudia

Saltasaurus

Isisaurus

Alamosaurus

Alamosaurus

Aeolosaurus

Rapetosaurus 


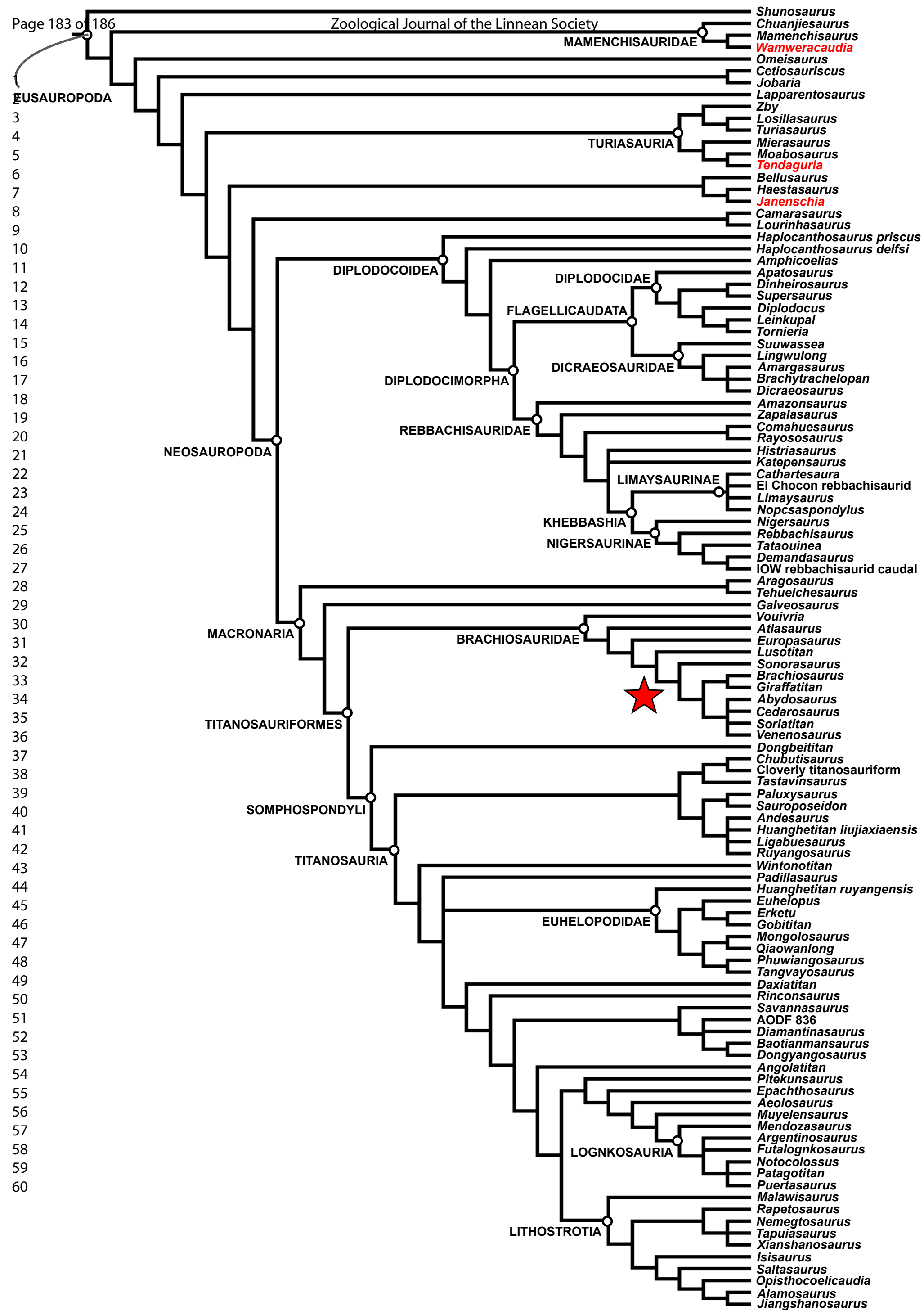




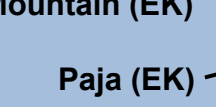

Rio Piranhas (EK)

Cañadón Asfalto (MJ)

Cañadón Calcáreo (LJ)

Bajada Colorada (EK)

La Amarga (EK)
Oxford Clay (MJ)

Wealden (EK)

Kirkwood (EK)

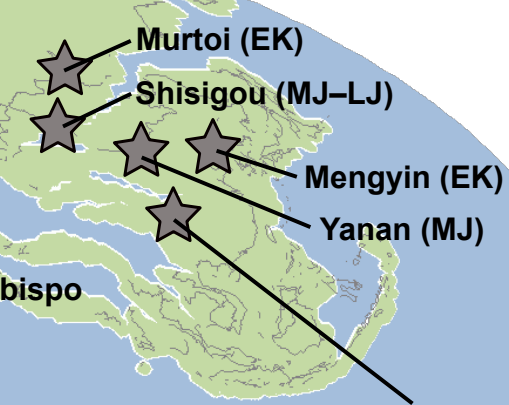

Shaximiao (MJ-LJ)
Tendaguru (LJ) Isalo III (MJ)

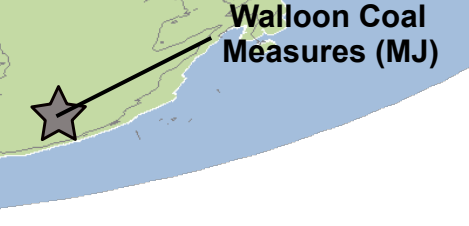

